\title{
Copyright
}

by

Terence A Campbell

2013 
The Dissertation Committee for Terence A Campbell Certifies that this is the approved version of the following dissertation:

\section{CORRECTION FOR DISTORTION IN POLARIZATION OF REFLECTED SHEAR-WAVES IN ISOTROPIC AND ANISOTROPIC MEDIA}

Committee:

Robert H. Tatham, Supervisor

Kyle Spikes

Diana Sava

Mrinal Sen

Clark Wilson 


\title{
CORRECTION FOR DISTORTION IN POLARIZATION OF REFLECTED SHEAR-WAVES IN ISOTROPIC AND ANISOTROPIC MEDIA
}

by

Terence A Campbell, B.S.; M.S.Geo.Sci.

\author{
Dissertation \\ Presented to the Faculty of the Graduate School of \\ The University of Texas at Austin \\ in Partial Fulfillment \\ of the Requirements \\ for the Degree of
}

Doctor of Philosophy

The University of Texas at Austin

December 2013 


\section{Dedication}

To my wife, the main anchor in my life when struggles arise and to my family. 


\section{Acknowledgements}

Dr. Robert H. Tatham, who was the major inspiration behind the project from the beginning, always gave words of wisdom and critique in order to inspire rather than dictate which allowed me to grow as an individual. Dr. Kyle Spikes who was a major beacon in a time of technical confusion a great friend and a major player in my geophysical endeavors.

Dr. Mrinal K. Sen gave me the theoretical background needed to delve into the mysteries of anisotropy, and Dr. Clark Wilson gave me timely and sage advice. I owe much to Dr. Bob Hardage of the Bureau of Economic Geology, who leads Exploration Geophysics Laboratory, for providing pivotal data and my voyage into post graduate research.

Dr. Diana Sava, Yi Tao and Mohammed Alhussain were also instrumental in helping reach the goals of this project. Last but not least I give much thanks to Thomas Hess because without his technical expertise and geophysical experience this project would not be where it is today. 


\title{
CORRECTION FOR DISTORTION IN POLARIZATION OF REFLECTED SHEAR-WAVES IN ISOTROPIC AND ANISOTROPIC MEDIA
}

\author{
Terence A Campbell, Ph.D. \\ The University of Texas at Austin, 2013
}

Supervisor: Robert H. Tatham

The progressive growth of onshore shale production (both gas and liquids) to replace depleting and aging oil fields may benefit from the use of surface seismic shear wave data analysis for full characterization of shale reservoir properties and lead to optimum development of these resources. This includes descriptions of azimuthal anisotropy (HTI - transverse isotropy with a horizontal symmetry axis) for characterization of fractures and internal fracture systems. The objective of this study is to document a predicted distortion in polarization of propagating seismic shear waves upon reflection at a subsurface interface and to propose a correction to this distortion. The polarization distortion occurs even in wholly isotropic media. This correction is based on an understanding of shear amplitude behavior as a function of the reflection incidence angle, particularly differences in the reflection angle relation for different shear components. This study includes a demonstration of the efficacy of the proposed correction by applying it to simulated and real direct shear-wave source data. Such corrections should result in a minimized polarization distortion in the reflection process. The apparent consistency of a null value (zero crossing) of the SV-SV reflectivity (near 20-24 degrees) for common density and velocity contrasts as well as the remarkably 
regular behavior of the SV-SV and SH-SH reflectivity curves following a linear relation in $\sin 2$ and $\tan 2$ of the incidence angle and offers the opportunity for a simple and stable correction with minimal sensitivity to detailed knowledge of contrasts in velocity and density. The only independent information required for the correction is the angle of incidence where the SV-SV and SH-SH reflections vanish and the range of these angles is typically quite limited.

Some key questions were addressed in gaining an understanding of shear wave polarization distortion upon reflection for varying model data: 1) how do we address reflected polarization distortion for purely isotropic medium for varying incidence angles? 2) How do we apply this correction for an isotropic and anisotropic media for both simulated and actual field data 3) How do we address applications to real data and how distorted amplitudes can be corrected to identify actual subsurface HTI anisotropy.

Significantly, the polarization distortion correction is implemented as a simple extension of the established Alford rotation for normal incidence shear reflections of varying polarization. This extension leads to the improved analysis of direct shear-source 3D data with inherently distorted polarization. Thus, analysis may be applied to estimate HTI anisotropy previously not realizable in finite offset data subject to polarization distortion. Example applications to actual field data are included.

Note that the polarization correction does remove the AVO effects often exploited in analysis of P-P data where polarization is not an issue that is, the AVO amplitude effect is essentially removed from the SV-SV and SH-SH oriented direct shear-wave profiles, which permits proper analysis of the polarization. Further, additional analysis of the polarization correction on field data with documented anisotropy will be required to fully develop the usefulness of this proposed correction. 


\section{Table of Contents}

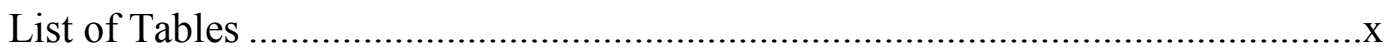

List of Figures .........................................................................................

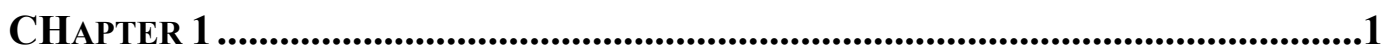

HYPOTHESIS, INTRODUCTION AND BACKGROUND ...............................................1

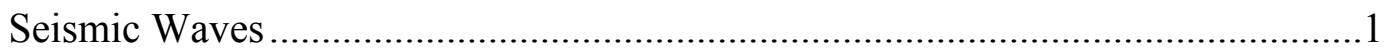

Acquisition of Direct Seismic Shear-Wave Data in 3D...................................6

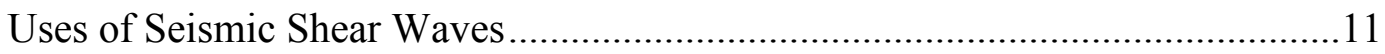

Optical Analogue ........................................................................... 13

Simulation of Polarization Distortion ............................................................ 18

Simplification of the SV and SH reflectivity relations ....................................26

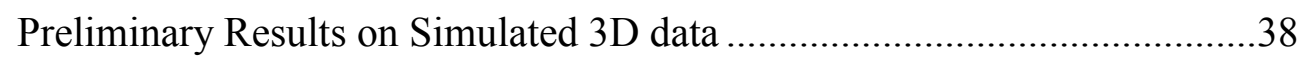

Analysis of Sensitivity of variations in the Zero-Crossings ..............................42

CHAPTER 2: ANISOTROPY .........................................................................................48

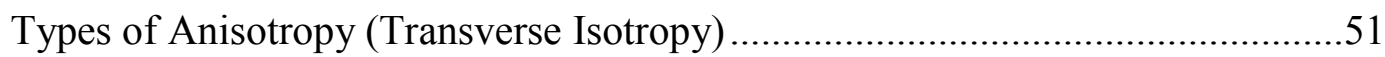

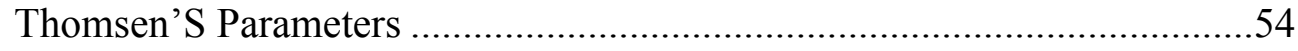

Shear waves and Azimuthal AVO in Transversely Isotropic Media .....................58

Zero-crossing using Rüger's Equation..................................................59

SIMULATED 3D FIELD RECORDS FOR SEISMIC SHEAR SOURCES ........66

CHAPTER 3: BLACK BEAR SURVEY, ARDMORE BASIN OKLAHOMA, USA.....85

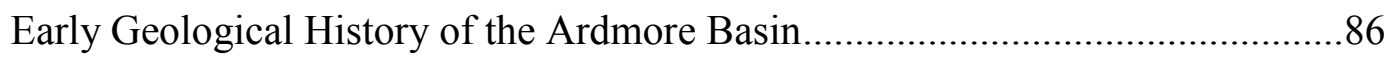

Stratigraphy of the Ardmore Basin .............................................................91

9-C VSP ACQUISITION IN THE TEXACO BRADY RANCH -1 Well ...95

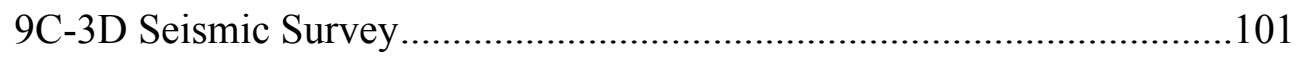


Seismic Acquisition Parameters for 3D-9C Survey............................................. 104

CMP Fold, Source-Receiver Offset and Source-Receiver Azimuth:...........106

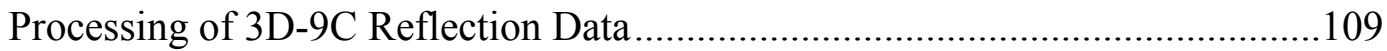

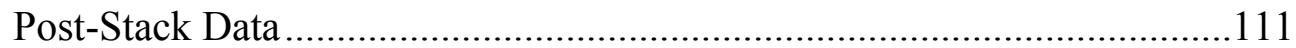

Polarization Analysis of the pre-stack data..................................................118

Polarization Observations and Correction Black Bear 3D 9C Seismic Data.......122

CHAPTER 4: ALFORD ROTATION ..............................................................................131

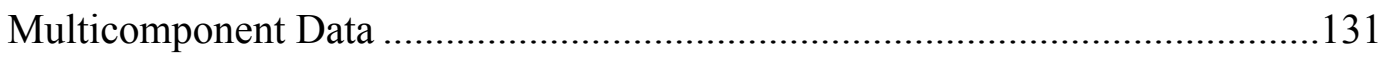

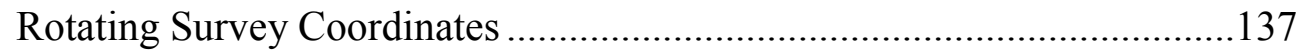

Vertical Incidence Direct Shear Waves and Alford Rotation ................................140

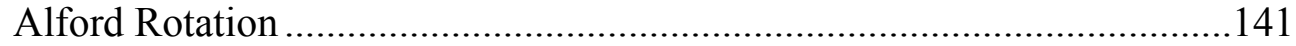

Polarization Correction to Isotropic Medium .....................................................142

Polarization Correction for the Anisotropic Medium ..........................................150

Alford Rotation Applied to BlackBear field Oklahoma ………...........................156

CHAPTER 5: DISCUSSION AND CONCLUSION .........................................166

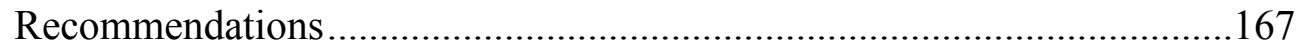

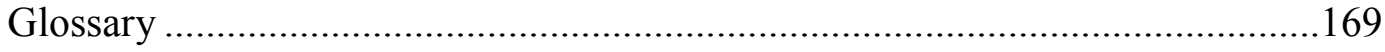

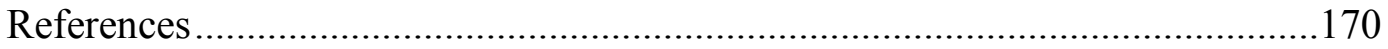




\section{List of Tables}

Figure 1: Particle motion during horizontal passage of plane body waves, (a) P-waves with particle motion parallel to the propagation direction and (b) Swaves when polarization is perpendicular to the propagation direction. Note that the particle motion of the propagating P-wave is in the direction of propagation so polarity is defined solely by the propagation direction. The particle motion of the propagating S-wave is orthogonal to the direction of propagation, so there is a wide variety, $\left(360^{\circ}\right)$ of polarizations for a given propagation direction (Sheriff and Geldart, 1993)

Table 1: Two layer isotropic model with elastic and seismic properties. 10

Table 2: Properties of anisotropic synthetic media, where $V_{P}$ is the compressional wave velocity, $\mathrm{V}_{\mathrm{S}}$ is the shear wave velocity. $\mathrm{V}_{\mathrm{P}}(0)$ and $\mathrm{V}_{\mathrm{S} 1}(0)$ are the vertically propagating $\mathrm{P}$-wave and $\mathrm{S} 1$ polarized shear wave velocities in the HTI azimuthally anisotrpic media, respectively. $\varepsilon, \delta$, and $\gamma$ are the exact Thomsen parameters of the equivalent HTI media. The only difference in the models 1, 2 and 3 is in the value of $\gamma$ in the lower layer. For Model 4, the anisotropy of the lower layer is only $1 \%$ essentially negligible 64 
Table 3: Properties of anisotropic (transversely isotropic) synthetic media, where Vp is the compressional wave velocity, Vs is the shear wave velocity. Vp (0) and Vs (0) are the vertically propagating p-wave and s-wave velocities in the HTI media, respectively. $\varepsilon, \delta$, and $\gamma$ are the exact Thomsen parameters of the equivalent HTI media. A range of $\gamma$ values are used to test its effect on the reflected waves. .74

Table 4: Table shows some of the survey statistics of the Sycamore Oklahoma Prospect. .105

Table 5: Properties of an anisotropic synthetic media, where $V_{P}$ is the compressional wave velocity, $V_{S}$ is the shear wave velocity. $V_{P}$ and $V_{S}$ are the vertically propagating P-wave and S-wave velocities in the HTI media, respectively. $\varepsilon, \delta$, and $\gamma$ are the exact Thomsen parameters of the equivalent HTI media. 150 


\section{List of Figures}

Figure 2: Ray propagation and polarization directions, with propagation shown by smaller arrows next to rays, and particle motion shown by two-headed arrows for (a) P-P, (b) SH-SH and (c) SV-SV waves (Tatham and McCormack, 1991). Note that P-wave particle motion is parallel to the propagation direction and in the vertical plane defined by the source and receiver position. SV particle motion is normal to the propagation direction and in the vertical plane. SH particle motion is normal to the propagation direction and normal to the vertical plane. The particle motion of the SH wave is always horizontal but the particle motion of SV wave, although in the vertical plane, is not, in general, horizontal. In general, a propagating shear wave will have an arbitrary polarization normal (transverse) to the propagation direction. For most calculations, the physics suggest that we generally address any shear-wave polarization in terms of orthogonal SV and SH components. .5

Figure 3: The effect of HTI anisotropy possible association with vertical fractures, on horizontal SV and SH wave- propagation. Note the relationship between fracture orientation and polarization of S-waves exiting the medium (Tatham and McCormack, 1991). In this example for a horizontal propagation direction, the velocity of the $\mathrm{SH}$ wave (S2, with particle motion across the fractures) is less than the velocity of the SV wave with the particle motion parallel to the fractures, S1. The faster wave (SV fir horizontal propagation) is referred to as $\mathrm{S} 1$, while the slower wave is

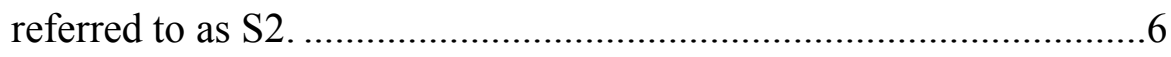


Figure 4: The acquisition geometry of a single source position in a 3D direct shear survey with a source polarization of $0^{\circ}$ (in general, a single horizontal shear source can have any polarization most direct-source 3D surveys utilizes two independent source polarizations, $\mathrm{X}$ and $\mathrm{Y}$ ) $\mathrm{L}$ is the observed polarization of the reflected wave is calculated. Note the significant derivation of the reflected shear-wave from the original source polarization (modified from Lyons, 2006).........................10 
Figure 5: Reflection coefficients for SV-SV (blue) and SH-SH (red) waves showing that the reflection coefficients vanish at some particular angle of incidence. Velocity and density information for all the examples shown is given in table 1.The position of the three critical incidence angles are shown. The three critical angles relate to the P-wave mode-converted and refracted wave into the lower layer $\left(22^{\circ}\right)$, the internally refracted mode-converted P-wave in the upper medium $\left(30^{\circ}\right)$ and the refracted Swave in the lower layer $\left(49^{\circ}\right)$. For this set of elastic and density parameters zero crossing is at $20^{\circ}$ for $\mathrm{SV}$ and $40^{\circ}$ for $\mathrm{SH}$. Note the constant phase of both SV-SV and SH-SH reflections before the first critical angle. Beyond the first critical angle which is beyond the SV-SV zero-crossing, there is a modest phase change for the SV-SV reflection but no phase change for the $\mathrm{SH}-\mathrm{SH}$ reflection until the one critical angle for the SH wave. Beyond the second critical angle of the SV-SV reflection the phase changes again from 40 degrees to 180 degrees to the third critical angle. The magnitude is shown as positive or negative in order that the phase will always be zero below the first critical angle, and as continuous as possible beyond that. The phase is always zero or pi in this region. 16 
Figure 6: The optical Brewster's angle (also known as the polarization angle) is an angle of incidence at which transversely polarized light, which is transversely polarized, with a particular polarization (equivalent to the seismic SV wave) is only transmitted through a surface, with no reflection. Interestingly, the ray-direction of the reflected wave aligns with the orientation of the equivalent SV component of the incident ray. For this angle the reflected wave is only polarized as an SH-wave thus the term "polarized angle". This angle is named after the Scottish physicist, Sir David Brewster (1781-1868). In the elastic case the randomly polarized S-wave is distorted upon reflection due to a deviation of reflection geometry from a purely SV and SH situation. In this example (as most optical examples), the lower medium has a lower velocity, the upper (air) medium. Thus, no critical angle exists and there is no phase distortion present. ..................................................... 17 
Figure 7: Simulated polarizations of shear-wave reflections in a single 3D source record (source at center) of a 3D survey of isotropic media (map view) corresponding to a reflector depth of $2000 \mathrm{~m}$. Two horizontal components (east and north) of particle motion are observed at receiver locations spatially distributed on the surface. The length of the vectors indicate the amplitude of the reflected wave, and the orientation of the vectors indicates the observed polarization (source is polarized due east). Note the wide variation in polarization over the surface. The red circle shows the offset associated with the $20^{\circ}$ zero-crossing of the SV reflection at an offset of about 1800 meters. The purple dashed lines shows the offset associated with the $40^{\circ}$ zero-crossing of the $\mathrm{SH}$ reflection at an offset at about 3000 meters. Note the change in polarization at the SV zero crossing position as source-receiver offset increases. Note the strong polarization distortion at increased offset.24 
Figure 8: Computed polarizations of shear-wave reflections in a single source record 3D survey of isotropic media (map view) corresponding to a reflector depth of $2000 \mathrm{~m}$. The length of the vectors indicate the amplitude of the reflected, and the orientation of the vectors indicates the observed polarization (source is polarized due north). Note that there is a change in SV polarity. Note the wide variation in polarization over the surface. The red circle shows the offset associated with the zero-crossing of the SV reflection. The purple dashed lines shows the offset associated with the zero-crossing of the SH reflection. Note the change in polarization at the zero crossing position. At large offsets (4200 meters) we only SV$\mathrm{SV}$ and no $\mathrm{SH}-\mathrm{SH}$ because it is equivalent to the $\mathrm{SH}-\mathrm{SH}$ zero crossing value angle. 25

Figure 9: Comparing the results of full Zoeppritz equations to a two term $\mathrm{Sin}^{2}$ approximation, describing SV-SV reflectivity. The model parameters are listed in Table 1. There is excellent agreement between the two through the zero crossing and a very useful agreement beyond that (modified Lyons, 2006). Note the eratic deviation and from standard Zoeppritz equation because of critical angles. 
Figure 10: Comparing the full Zoeppritz equations to a two term $\sin ^{2}$ approximation, describing SH reflectivity. The model parameters are listed in Table 1. The agreement to angles of incidence of $25^{\circ}-30^{\circ}$ is very good, but the agreement is poor beyond that and does not agree the zero crossing (elastic Brewster's angle). The lack of agreement between the two models after the zero offset reflectivity above $30^{\circ}$ is not at all useful considering we use the zero-crossing to make the correction (modified Lyons, 2006)... 30

Figure 11: Comparing the full Zoeppritz equations to a two term $\operatorname{Tan}^{2}$ approximation, describing SH motion. The model parameters are listed in Table 1. Good agreement out to the zero-crossing $40^{\circ}$ suggests this is a useful approximation (modified from Lyons, 2006). Note the divergence beyond $40^{\circ}$ especially beyond the critical angle $\left(48^{\circ}\right)$......................32 
Figure 12: Comparing the full Zoeppritz equations to a two term $\mathrm{Sin}^{2}$ approximation, describing SV motion, as well as correcting the SV reflectivity for variations in amplitude with angles of incidence. The parameters for the Zoeppritz curve are listed in Table 1. There is acceptable agreement between the full Zoeppritz and Spratt approximation through an incident angle of about $30^{\circ}$. The small departure at $\mathrm{j}=22^{\circ}$ corresponds to the critical angle for the first refracted P-wave in the second layer. There are also critical angles at $30^{\circ}$ and $49^{\circ}$ corresponding to the internal refraction in the upper layer and the shear wave refraction in the lower layer. The suggested corrections are also applied to the actual Zoeppritz curve. The unstable region near the zero crossing is omitted. The red line is the corrected reflection amplitude for the Spratt's approximation and the region between $18^{\circ}$ to $22^{\circ}$ are removed because the values are too large due to the singularity at $22^{\circ}$. The black dashed line is a horizontal line showing the removal of the AVO effect of the corrected reflection amplitudes. .36 
Figure 13: Comparing the full Zoeppritz equations to a two term tan approximation, describing SH reflectivity. The model parameters are listed in Table 1. There is good agreement between the actual Zoeppritz and $\operatorname{Tan}^{2}$ approximation through an incident angle of $40^{\circ}$. The suggested corrections are also applied to the actual Zoeppritz curve. The correction is omitted near the zero crossing. The red line is the corrected reflection amplitude for the Lyon's too approximation and the region above $40^{\circ}$ are removed because the values are too large due to the singularity at $40^{\circ}$. The black dashed line is a horizontal line showing the removal of the AVO effect of the corrected reflection amplitudes. .37

Figure 14: Polarization corrections applied to the simulated polarization distortions in a 3D record of isotropic media (map view) corresponding to a reflector depth of $2000 \mathrm{~m}$. The original polarization data is shown in Figure 7. The length of the vectors indicate the amplitude of the data, and the orientation of the vectors indicates the observed polarization (source is polarized due east). The corrections used a zero crossing of $22^{\circ} \mathrm{SV}$ and $40^{\circ} \mathrm{SH}$ from figures 12 and 13 for actual velocities used in the modeling. The purple dashed lines shows the offset associated with the zero-crossing of the SH reflection. 
Figure 15: This plot is similar to Figure 14 with Polarization corrections applied to the simulated polarizations in a 3D survey of isotropic media (map view) corresponding to a reflector depth of $2000 \mathrm{~m}$. The original polarization data is shown in Figure 8. The length of the vectors indicate the amplitude of the corrected data, and the orientation of the vectors indicates the corrected polarization (source is polarized due north). The purple dashed lines shows the offset associated with the zero-crossing of the SH reflection. The corrections used a zero crossing of $20^{\circ} \mathrm{SV}$ and $40^{\circ} \mathrm{SH}$ from figures 12 and 13 for actual velocities used in the modeling. The only differences in Figure 14 and Figure 15 are the input source polarization correction.

Figure 16: $\mathrm{R}_{\mathrm{SV}-\mathrm{SV}}$ reflection coefficient for incidence angles of $0^{\circ}$ to $60^{\circ}$ with changes in shear wave velocity contrast of $+/-25 \%$. Initial $\mathrm{V}_{\mathrm{P}} / \mathrm{V}_{\mathrm{S}}=1.8$, density $=$ $2.2 \mathrm{~g} / \mathrm{cc}$; density and $\mathrm{V}_{\mathrm{P}}$ is held constant and the shear wave velocity varies $+/-25 \%$ (follows Lyons (2006) Figure 3.9). Note the effect of critical angles at large contrasts in Vs. Also note the consistency of the zero-crossing near about $22^{\circ}+/-2^{\circ}$. 44

Figure 17: $\mathrm{R}_{\mathrm{SH}-\mathrm{SH}}$ reflection coefficient with changes in shear wave velocity contrast. Initial $\mathrm{V}_{\mathrm{P}} / \mathrm{V}_{\mathrm{S}}=1.8$, density $=2.2 \mathrm{~g} / \mathrm{cc}$; density and $\mathrm{V}_{\mathrm{P}}$ is held constant and the shear wave velocity varies $+/-25 \%$. The curves are quite similar with zero crossing in the range of $40^{\circ}+/-5^{\circ}$ (follows Lyons (2006) Figure 3.10). For the $\mathrm{SH}-\mathrm{SH}$ reflector, the reflection coefficient is independent of $\mathrm{V}_{\mathrm{P}}$ but is dependent on density and shear wave velocity. 45 
Figure 18: $R_{S V-S V}$ reflection coefficient with changes in density contrast. Initial $V_{P} / V_{S}$ $=1.8$, density $=2.2 \mathrm{~g} / \mathrm{cc} ; \mathrm{V}_{\mathrm{P}}$ and $\mathrm{V}_{\mathrm{S}}$ is held constant and the density varies $+/-25 \%$. The value of the zero-crossing is essentially independent of the density. 46

Figure 19: $\mathrm{R}_{\mathrm{SH}-\mathrm{SH}}$ reflection coefficient with changes in density contrast. Initial $\mathrm{V}_{\mathrm{P}} / \mathrm{V}_{\mathrm{S}}$ $=1.8$, density $=2.2 \mathrm{~g} / \mathrm{cc} ; \mathrm{V}_{\mathrm{S}}$ and $\mathrm{V}_{\mathrm{P}}$ is held constant and the density varies $+/-25 \%$. The curves are quite similar in character with no zerocrossing. 47

Figure 20: Model of shear wave splitting, or birefringence. An arbitrarily polarized shear wave enters the HTI (vertically fractured) medium vertically from above. Within the medium one component of the shear wave is polarized in the direction of the fractures and becomes S1, or the fast shear wave. The remaining orthogonal component is polarized in the direction perpendicular to the fracture orientation and becomes the slow shear wave, or S2 (modified from Bowman et al., 1987 and Martin and Davis, 1987). Thus, the incoming wave is split into two polarizations; this is identical to optical birefringence. Since the waves travel at different velocities, they separate leading to a time delay. This time delay may be related to the fracture intensity (Tatham and McCormick, 1991) ....50

Figure 21: Sketch of VTI and HTI models. Reflections of P-waves confined to the two vertical symmetry planes, here called the symmetry-axis plane and the isotropy plane. Shear waves polarized parallel and normal to the isotropy plane have different vertical velocities (Rüger, 1997).......54 
Figure 22: Sketch of the geometry associated with an isotropic layer over an HTI model. Reflections of S-waves polarizations confined to the two vertical symmetry planes, here called the symmetry- axis plane and the isotropy plane, are discussed in the text. Vertically propagating shear waves polarized parallel and normal to the isotropy plane have different vertical velocities in the lower (HTI) medium, but equal velocities in the upper isotropic medium (Rüger, 1996). .57

Figuüe 23: Plan view of HTI medium, with the source and receiver acquisition profiles fracture strike and HTI symmetry direction and the polarization direction for the fast and slow shear waves. Amplitude analysis as a function of angle of incidence in the isotropic layer will be performed in the strike and symmetry profile directions which represent the isotropy and the symmetry-axis planes, respectively. In this nomenclature $\mathrm{S} \|=$ $\mathrm{S} 1$, the polarization of the fast shear wave, $\mathrm{S} \perp=\mathrm{S} 2$, the polarization of the slow shear wave (Rüger, 1996) 63 
Figure 24: Values of the zero-crossing for SV-SV and SH-SH reflection coefficients as a function of incidence angle for two different models of an isotropic layer over an HTI medium. The layer properties are given in Table 2 and the graphs are calculated using Rüger's equation for isotropic over transversely isotropic (HTI) medium. Note zero crossings values for the various models which are used to identify singularities in the polarization correction analysis. In the top graphs the source is oriented parallel to fractures and in the lower plots the source is oriented perpendicular to fractures. Note similarity in the value for and $\mathrm{SH}-\mathrm{SH}$ and SV-SV zero crossing values for a large range of $\gamma$ values. This is consistent with an almost constant value for zero-crossing for S1 and S2 polarizations for varying acquisition geometries.

Figure 25: Acquisition geometry of the synthetic models. The two horizontal sources are situated at the center of the receiver positions. Receivers are positioned every $100 \mathrm{~m}$ in both $\mathrm{X}$ and $\mathrm{Y}$ directions with the source located at $(0,0)$. The black arrows indicate the N-S and E-W receiver orientations and the red arrows indicate N-S and E-W source orientations. Each shot has 625 pairs of observer N-S and E-W receiver traces. Each of the 625 receiver location can be resolved into a recorded shear-traces polarization and amplitude for each of the two source polarizations. 
Figure 26: Observed (simulated) polarizations in a single source record (X-oriented source at center) of a 3D survey (map view) for a reflector depth of $2000 \mathrm{~m}$. HTI fracture orientation in the lower medium is E-W $\left(90^{\circ}\right.$ west of north). The circle indicated the offset corresponding to an incidence angle of $22^{\circ}$ (the SV-SV zero crossing) at an offset of about $1400 \mathrm{~m}$. The $50^{\circ} \mathrm{SH}-\mathrm{SH}$ zero crossing is at $2400 \mathrm{~m}$. The length of the vectors indicates the amplitude of the recorded data, and the orientation of the vectors indicates the observed polarization. Thomsen parameters used are; Gamma $=0.05$, Delta $=0.10$, Epsilon $=0.30$. The upper layer is isotropic. Note consistent E-W polarization of the reflected shear-waves at offsets up to at least 500 meters, and almost to the SV-SV zero crossing. Also note the rather strong distortion of polarization just beyond the SV-SV zero crossing, especially source-receiver azimuths at $45^{\circ}$ (ellipse), including the high amplitude and strong distortion at about 1500 meters well before the SH-SH zero crossing. .75 
Figure 27: Polarization correction applied to the simulated polarizations (Figure 26) in a single $3 \mathrm{D}$ source $3 \mathrm{D}$ record (X-oriented source at center) of a $3 \mathrm{D}$ survey (map view) for a reflector depth of $2000 \mathrm{~m}$. The circle indicated the offset corresponding to an incidence angle of $22^{\circ}$ (the SV-SV zero crossing). The length of the vectors indicates the amplitude of the recorded data, and the orientation of the vectors indicates the observed polarization. Fractures are oriented east - west. Thomsen parameters used are; Gamma $=0.05$, Delta $=0.10$, Epsilon=0.30. The SH-SH zero crossing is at 2400 meters. Note the consistency of the corrected polarization, other than in SV-SV zero-crossing gap, out to 1400 meters and beyond. Now we see below the SV-SV zero-crossing all the reflected polarizations are correctly oriented. Beyond the SV-SV zero crossing (ellipse) the reflected polarizations are also correctly define the

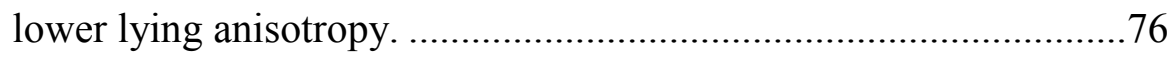


Figure 28: Observed (simulated) polarizations for the same source and receiver geometry and medium properties as Figure 27 but the fractures are oriented $45^{\circ}$ east of north. Note the consistency of the vectors at the fracture strike direction of $45^{\circ}$. This is consistent with the S1 polarization of the lower layer. This polarization is observed at zerooffset, suggesting that the lower medium controls the direction of polarization. Beyond the SV-SV zero-crossing at $1400 \mathrm{~m}$, the polarizations become quite different and quite pronounced beyond the $\mathrm{SH}-\mathrm{SH}$ zero-crossing. Thomsen parameters used are; Gamma $=0.05$, Delta $=0.10$, Epsilon $=0.30$. Note the observed (simulated) polarizations in a single $3 \mathrm{D}$ source record (X-oriented source at center) of a 3D survey (map view) for a reflector depth of $2000 \mathrm{~m}$. The circle $(1400 \mathrm{~m})$ indicated the offset corresponding to an incidence angle of $22^{\circ}$ (the SV-SV zero crossing). The purple circle shows the SH-SH zero crossing at $2400 \mathrm{~m}$. 
Figure 29: Polarization correction applied to Figure 29. Note the resultant vector arrows are oriented with the same direction as the medium anisotropy for a single 3D source record (X-oriented source at center). The correction results in the orientation with substrate fracture direction rather than the source direction. The correction loos great all the way to the SH-SH zero crossing but the correct fracture orientation rather than the source direction (as in the isotropic case). Reflector depth is at 2000m. The circle indicated the offset corresponding to an incidence angle of $22^{\circ}$ (the SV-SV zero crossing). The length of the vectors indicates the amplitude of the recorded data, and the orientation of the vectors indicates the observed polarization. Fractures are oriented $\mathrm{N} 45^{\circ} \mathrm{E}$ ( northeast - southwest). Thomsen parameters used are; Gamma $=0.05$,

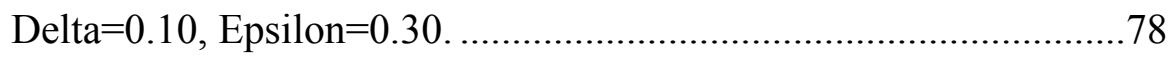


Figure 30.A: Simulated data for same acquisition geometry as Figure 27 and 29 but for decreased anisotropy $(\gamma=2 \%)$, a north $(Y)$ oriented horizontal source and HTI (fracture) strike of $\mathrm{N} 45^{\circ} \mathrm{W}$. Note the observed (simulated) polarizations in a single 3D source record ( $\mathrm{Y}$-oriented source at center) of a 3D survey (map view). Note the change in the polarization direction from Figure 28 the input source polarizations are different which causes the reflected polarization direction to change but still properly define the HTI anisotropy. The reflected polarization defines the underlying anisotropy at offsets less than the SV-SV zero crossing. The circle indicated the offset corresponding to an incidence angle of $22^{\circ}$ (the SVSV zero crossing). The length of the vectors indicates the amplitude of the recorded data, and the orientation of the vectors indicates the observed polarization. Fractures are oriented $\mathrm{N} 45^{\circ} \mathrm{W}$ (northwest southeast). Thomsen parameters used are; Gamma $=0.02$, Delta $=0.10$, Epsilon $=0.30$. 
Figure 30.B: Polarization correction applied to Figure 30A. We can see it also has the correct fracture direction at offset up to at least the SV-SV zero crossing. Note we muted values around the zero crossing due to the singularity. The reflected polarizations correctly define the lower layer anisotropy even slightly beyond the SV-SV zero crossing. We had to mute out several values before the SH-SH zero crossing because the values were quite large in comparison to the other polarization values. The circle indicated the offset corresponding to an incidence angle of $22^{\circ}$ (the SVSV zero crossing). The length of the vectors indicates the amplitude of the recorded data, and the orientation of the vectors indicates the observed polarization. Fractures are oriented $\mathrm{N} 45^{\circ} \mathrm{W}$ (northwest southeast). Thomsen parameters used are; Gamma $=0.02$, Delta $=0.10$, Epsilon $=0.30$ 80

Figure 31.A: Figure used to simulate a $10 \%$ gamma anisotropic medium and it was observed that no matter the source orientation the observed polarization is properly oriented. This mimics observed (simulated) polarizations in a single 3D source record (X-oriented source at center) of a 3D survey (map view) for a reflector depth of $2000 \mathrm{~m}$. The circle indicated the offset corresponding to an incidence angle of $22^{\circ}$ (the SV-SV zero crossing). Note the zero crossing value at an X offset of 1350 meters corresponding to $22^{\circ}$. The length of the vectors indicates the amplitude of the recorded data, and the orientation of the vectors indicates the observed polarization. Fractures are oriented east-west. Thomsen parameters used are; Gamma $=0.10$, Delta $=0.10$, Epsilon $=0.30 \ldots . .81$ 
Figure 31.B: Polarization correction applied toFigure $32 \mathrm{~A}$ where the corrected polarization applied to the simulated data in a single 3D source record (Y-oriented source at center) of a 3D survey (map view) for a reflector depth of $2000 \mathrm{~m}$. The circle indicated the offset corresponding to an incidence angle of $22^{\circ}$ (the SV-SV zero crossing). Note the observed polarization is corrected oriented before and after the polarization correction is applied. So it can be said no matter the source orientation once gamma is greater than $10 \%$ we can define fracture strike. The length of the vectors indicates the amplitude of the recorded data, and the orientation of the vectors indicates the observed polarization. Fractures are oriented east - west. Thomsen parameters used are; Gamma $=0.10$, Delta $=0.10$, Epsilon $=0.30$.

Figure 31.C: Figure used to simulate a $10 \%$ gamma anisotropic medium and it was observed that no matter the source orientation the observed polarization is properly oriented. It mimics observed (simulated) polarizations in a single 3D source record (X-oriented source at center) of a 3D survey (map view) for a reflector depth of $2000 \mathrm{~m}$. The circle indicated the offset corresponding to an incidence angle of $22^{\circ}$ (the SV-SV zero crossing). Note the zero crossing value at a X offset of 1250 meters corresponding to $22^{\circ}$. The length of the vectors indicates the amplitude of the recorded data, and the orientation of the vectors indicates the observed polarization. Fractures are oriented $\mathrm{N} 30^{\circ} \mathrm{E}$ (northeast southwest). Thomsen parameters used are; Gamma $=0.10$, Delta $=0.10$, Epsilon $=0.30$. Note the polarization correctly defines the lower layer anisotropy even beyond the SV-SV zero-crossing. 
Figure 31.D: Corrected polarization plot from Figure $32 \mathrm{~A}$ where the corrected polarization applied to the simulated data in a single 3D source record (Y-oriented source at center) of a 3D survey (map view) for a reflector depth of $2000 \mathrm{~m}$. The circle indicated the offset corresponding to an incidence angle of $22^{\circ}$ (the SV-SV zero crossing). The correction results in the orientation with substrate fracture direction rather than the source direction. The correction loos great all the way to the SH-SH zero crossing but the correct fracture orientation rather than the source direction (as in the isotropic case). Note the observed polarization is corrected oriented before and after the polarization correction is applied. So it can be said no matter the source orientation once gamma is greater than $10 \%$ we can define fracture strike. The length of the vectors indicates the amplitude of the recorded data, and the orientation of the vectors indicates the observed polarization. Fractures are oriented $\mathrm{N} 30^{\circ} \mathrm{E}$ (northeast - southwest). Thomsen parameters used are; Gamma $=0.10$, Delta $=0.10$, Epsilon $=0.30$. .84

Figure 32: Generalized distribution of major geologic provinces of Oklahoma. The Ardmore Basin is in south-central Oklahoma, mostly in Marshall, Carter and eastern Stephens Counties. The red outline represents the location of the Black-Bear survey. The Texaco Brady 1 well is about $30 \mathrm{~km}$ east of the Black-Bear survey, shown by the red circle (modified from, Johnson, 2008). .86 
Figure 33: Structural development of the Southern Oklahoma aulacogen source. A: Middle Cambrian extension, faulting (rifting) of the granite basement and filling of rift with volcanic rocks. B: Late Cambrian to Early Devonian subsidence and accumulation of mostly marine limestone and to a lesser extent sandstone and shale. C: Continued subsidence in the Late Devonian to Late Mississippian and deposition of mostly marine shale and minor sandstone and limestone. D: Folding and faulting during Wichita and Arbuckle orogenies during Pennsylvanian and Permian time. Note the top of the local Pennsylvanian angular unconformity at the base of the conglomerate that formed as a result of the Arbuckle orogeny (Kleehammer, 1991). The top of the regional Mississippian unconformity occurs below the top of the Pennsylvanian an in this section is in very complex folded region. The top of the Mississippian is observable on the seismic section. The Top of the Mississippian unconformity is regional across North America (including Alberta) 89

Figure 34: Late Paleozoic structures of the Washita Valley wrench fault system and tectonic setting of the Ardmore basin, Oklahoma also shown is the location of the 16 sq. miles seismic survey and the location of the outer well (Brady-1 well) (modified from, Harding, 1983). 
Figure 35: Generalized surface and subsurface stratigraphic columns for major sedimentary basins in Oklahoma. Formal stratigraphic names in standard print informal unit names in italics. Formal formation names are not necessarily differentiated. Local informal nomenclature from Pennsylvanian units varies in the southern Anadarko Basin. Double columns of informal names indicate different local usage and unknown correlation of units. The area of black circles represents relative cumulative gas production. Compiled primarily from Cipriani (1963), Hills and Kottlowski (1983), Mankin (1986). Other sources are Amsden (1979, 1980, 1989), Amsden and Sweet (1983), Johnson (1988), Hester and others (1990), Baars and others (1991), Carpenter and Evans (1991), Davis and Northcutt (1991), Wilson and others (1991), Knode and

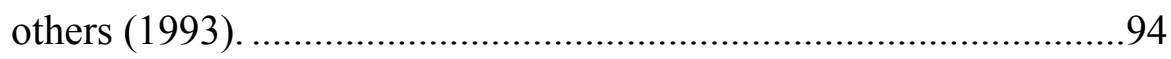

Figure 36.A: Orientation of azimuthal anisotropy curves versus depth from analysis of Brady Ranch 1 VSP data. The dashed curve is a result of using only Synchronous Rotation(SR) independently at each depth level. The solid curve is the result of applying the downward continuation algorithm plus SR. This curve is useful to estimate orientation of vertical fractures at a given depth. Note the abrupt change at the top of the Mississippian unconformity (Beckham, 1996). 99

Figure 36.B: Time delay between fast and slow split shear waves from analysis of the Brady Ranch 1-5 VSP data. The dashed curve is the result of applying Synchronous Rotation at each depth level, and the solid curve is the result of applying downward continuation plus SR. The time delay is often used as a proxy for fracture intensity (Beckham, 1996).........99 
Figure 37. Diagram showing orientation of azimuth anisotropy as obtained from cross-dipole log data analysis in specific depth intervals below $2700 \mathrm{~m}$ $(9,000 \mathrm{ft})$ in the carbonate section. Orientations from the VSP data are also indicated (Beckham, 1996). 100

Figure 38: Google Earth image showing the surface Ardmore Basin in Oklahoma with the different counties and fluvial distributaries. The red box outlines the area of the BlackBear survey and the blue circle is the Texaco Brady Ranch 1-5 well multicomponent VSP and cross-dipole shear wave $\log .$. 102

Figure 39: Diagram of Source and 3-C Receiver pairs. The direct primary wave data were acquired using an explosive source but the S-wave wave data was acquired using Vibroseis sources. Total surface area covered is about 16 square miles. Source lines are about 350 meters apart and receiver lines are 420 meters apart. Note the concentration of seismic source locations to the southern portion of the survey, limiting the concentration of high quality reflection data to that area. 103

Figure 40: A plot of CMP fold showing 360-fold coverage over the center of the survey for an X Source - X Receiver orientations. The edge of the survey has lower fold because several of the first and last shots do not reach as many receivers as in the central part of the survey. 107

Figure 41: Histogram showing the source-receiver offset distribution over the entire survey. Note the different offset distribution between 2000-9000 ft.108

Figure 42: An azimuth distribution histogram showing the number of traces over the entire survey not for each CMP with the associated its azimuths...108

Table 5: Direct shear-wave processing flow. 110 
Figure 43: 3D stacked P-wave profile showing structure. Note the angular unconformity (Top Mississippian Unconformity) at approximately 1.25 sec and the strong reflection range at $0.5 \mathrm{sec}$ which was used as the layer for polarization distortion correction. Note: Deterioration of data quality below the top of Mississippian and at Sycamore levels. Also note good quality shallow data above $1000 \mathrm{msec}$

Figure 44: 3D stacked S-wave $(\mathrm{SH})$ profile showing equivalent structure to the $\mathrm{P}$ wave data with lower seismic resolution in reflection time. When converted to depth the resolution with $\mathrm{P}$ is probably comparable. Note the unconformity at approximately $2.8 \mathrm{sec}$ and the strong reflection at $1.1 \mathrm{sec}$ which is equivalent to the P-wave surfaces that were identified. The deeper events are used in the registration not in the correction. Note: Deterioration of data quality below the top of Mississippian and at Sycamore levels. Also note good quality shallow data

Figure 45: 3D image of profile showing both the $\mathrm{P}$ wave and $\mathrm{SH}$ wave profile where the resolution and structure can be quickly identified and reflections visually correlated between the $\mathrm{P}$ and $\mathrm{S}$-wave $(\mathrm{SH})$ data. Note the differences in reflection time between the two volumes, which are adjusted for difference in $\mathrm{P}$ and $\mathrm{S}$ propagation velocities and the structural complexity deeper in the section that is seen on the P-wave volume is not seen on the S-wave volume. Below the Mississippian unconformity on the P-wave seismic data we can still identify stratigraphy and structural complexity. Note below the Mississippian unconformity on the S-wave data we cannot see the same seismic resolution so interpretation is very difficult. 
Figure 46: 3D display of P-wave reflecting horizon showing little structural relief ranging from $456 \mathrm{msec}$ to $536 \mathrm{msec}$. The warm colors are shallower and the cool colors are deeper. The surface is dipping from North to South. 3D structure map of the shallow event on better image quality P-wave data. 116

Figure 47: 3D time structure of S-wave (SH) horizon showing little structural relief ranging from $976 \mathrm{msec}$ to $1144 \mathrm{msec}$. The warm colors are shallow and the cool colors are deep. The surface is dipping from North to South. Note the "streaks" in the data associated with the acquisition footprint on the structure map which is very common in S-wave data acquisition and results from insufficient surface sampling at this relatively shallow depth. Note difference in reflection time for a common P and S-wave (SH) reflector, due to velocity differences. 117

Figure 48: Single source position and orientation in the $\mathrm{X}$ and $\mathrm{Y}$ oriented receiver grid with a selection of associated $3 \mathrm{C}$ receiver locations. Note the wide range of azimuths and offsets for source-receiver picks associated with the single source position. In the total survey, many more source and receiver positions are available. The red lines are the in-line direction between the source and equivalent receiver used to calculate azimuth. The maximum source-receiver offset in this set is about 3500 feet. 120 
Figure 49: Single source position (source) gathers from the single $\mathrm{X}$ recorded trace (east) oriented shear source shown in Figure 48and recorded by X (east) oriented receiver organized by source-receiver offsets. Note the single source data we sorted by offset so the polarization correction can be applied. Each trace has a different source-receiver azimuth. The reflector (strong on the stacked volume) at $1050 \mathrm{msec}$ on the stacked section is identified. The polarization correction will be applied to this reflector, so this offset represents some large reflection incidence angles. Note that the reflection quality is best at source-receiver offset up to about $4000 \mathrm{~m}$. The reflector is at a depth of about 600 meters............................121 
Figure 50: Observed reflection polarization for a single shear source oriented due east (source at center and six receiver lines as shown in Figure 42) of the 3D 9C BlackBear survey from a single reflector at $1050 \mathrm{msec}$. The blue crosses represent the receiver locations. Both X (east) and Y (North) oriented receivers are used to determine the observed polarization of the reflected event. The length of the vectors indicates the amplitude of the recorded reflection, and the orientation of the vectors indicates the observed polarization. Due to the polarity reversal for shear-wave reflections at or normal incidence where there is no polarization distortion, the reflection polarization would be in the $\mathrm{X}$ (east) direction for purely isotropic material. Note the regional fracture orientation is expected to be $30^{\circ}$ northeast-southwest. Source-receiver offset the distance from the source point at center, to the receiver location. Essentially all of the receiver locations observe purely S1 polarizations at the shallow section parallel to the fracture direction consistent with the presumed anisotropy of the upper medium the wave propagate through. 
Figure 51: Corrected polarizations applied to the observed polarization distortions for a single shear source oriented due east (source at center and six receiver lines as shown in Figure 42) of the 3D 9C BlackBear survey. Blue crosses represent the receiver locations. The length of the vectors indicates the amplitude of the recorded reflection, and the orientation of the vectors indicates the observed polarization. Note the similarity between the observed and corrected polarizations. Note Offset means the distance from the center at the source point. The similarity between the polarization after correction for assumed distortion and the observed polarization aligned with the HTI symmetry suggests that the polarization correction does "minimal" damage to the observed polarization. 128 
Figure 52: North oriented source for a observed reflection polarization for a single shear source oriented due north (source at center and six receiver lines as shown in Figure 42) of the 3D 9C BlackBear survey from a single reflector at $500 \mathrm{msec}$. The blue crosses represent the receiver locations. Length of the vectors indicates the amplitude of the recorded reflection, and the orientation of the vectors indicates the observed polarization. For the case of no polarization distortion, the reflection polarization would be in the $\mathrm{X}$ (east) direction. Note the fracture direction for east oriented source and north oriented source are the same because it's the same reflector level. Source-receiver offset is the distance from the center at the source point. Due to the difference in source direction than Figure 50 we see slight difference in the observed polarization. Note at offsets $\geq$ $4000 \mathrm{~m}$ the fracture direction of the reflected polarization is better determined than a source due east (Figure 50). .129 
Figure 53: Corrected Polarization applied to the observed polarization distortions for a single shear source oriented due north (source at center and six receiver lines as shown in Figure 42) of the 3D 9C BlackBear survey for a single reflector $500 \mathrm{msec}$. The blue cross represents the receiver locations. Both X (east) and Y (North) oriented receivers are used to determine the actual polarization of the reflected event. The length of the vectors indicates the amplitude of the recorded reflection, and the orientation of the vectors indicates the observed polarization. For the case of no polarization distortion, the reflection polarization would be in the $\mathrm{X}$ (east) direction. Note the fractures are oriented $30^{\circ}$ northeastsouthwest. Note at higher percentages of anisotropy (seen in the Brady-1 well), the resultant reflected polarization map still correctly defines the HTI anisotropy (as shown). This indicated that no matter how the source is oriented in the east or north direction we can properly define the fracture. At far offsets the fracture direction can be identified but it may be more difficult as seen in Figure 50 and Figure 52.. Note Offset means the distance from the center at the source point. 130 
Figure54.CMP gathers in survey coordinates as a function of source-receiver azimuth. Each set of 4 panels represent polarization of source-receiver pairs: $\mathrm{SxRx}-\mathrm{X}$ oriented source $\mathrm{X}$-oriented receiver, SxRy $-\mathrm{X}$ oriented source Y oriented receiver, SyRx - Y oriented source X oriented receiver, SyRy - Y oriented source Y oriented receiver. The data are full-prospect "super gathers" (sums of all CMP bin gathers from the entire survey) in survey coordinates as a function of source-receiver azimuth. Data are displayed in two-way reflection time in seconds and offset, in $\mathrm{km}$. Note each panel has an offset from 0 to $3 \mathrm{~km}$ and it is NMO corrected at a constant shear-wave velocity. Source receiver offset increases from $0-3$ $\mathrm{km}$ to the right in each subpanel. Source-receiver azimuth is noted in the upper right of each panel and offset, in $\mathrm{km}$ is indicated at the bottom of

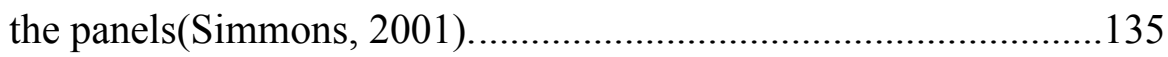

Figure 55: Data from Figure 54 after rotation into radial and transverse components for each source-receiver pair. The cross-term energy is at minimum and the $\mathrm{SV}$ and $\mathrm{SH}$ data is isolated in the $\mathrm{S}_{\mathrm{R}}-\mathrm{R}_{\mathrm{R}}(\mathrm{SV})$ and $\mathrm{S}_{\mathrm{T}}-\mathrm{R}_{\mathrm{T}}(\mathrm{SH})$ components. The data are full-prospect "super gathers" (sums of all CMP bin gathers from the entire survey) in survey coordinates as a function of source-receiver azimuth. Data are displayed in time and they are NMO corrected. Note each panel has an offset from 0 to $3 \mathrm{~km}$. Source receiver offset increases from 0 to $3 \mathrm{~km}$ to the right in each subpanel. Source-receiver azimuth is noted in the upper right (Simmons, 2001) 136 
Figure 56: Description of coordinate systems used in rotation analysis of horizontal components for both 9C (3-source and 3-receiver components) and 3C (1 source and 3 receiver components) acquisition. Panels (a) and (b) show a single source position and three receiver positions in plan view, with the red arrows showing source-receiver azimuthal paths for both the 9C case (a) and the 3C P-SV mode converted case (b). Note that X is upward and Y is to the left this particular "survey-parameter" orientation. For the 9C case both source and receiver polarizations are aligned in uniform $\mathrm{X}$ and $\mathrm{Y}$ directions defined by the acquisition survey parameters. The $3 \mathrm{C}$ case is similar but with a compressional (vertical, or Z, polarization) source. Panels (c) and (d) illustrate the resultant source and receiver orientations after trigonometric rotation of the recorded horizontal components to radial and transverse coordinate. For the 9C case (c) the source and receiver polarizations are both rotated to the azimuth direction. For the $3 \mathrm{C}$ case (d) where there are no horizontal source components, only the receiver polarizations are defined by the source-receiver positions, rotated to radial and transverse components. In figures (e) and (f) the underlying background lines represent parallel joints or cracks typical of an HTI (transverse isotropy) medium. Where appropriate the horizontal source and receiver polarizations are aligned in preferential S1 and S2 orientations of the fractures (Gumble, 2006). 
Figure 57: Synthetic data on a X-oriented time distance profile for a two layer isotropic model outlined in Table 1. Layer depth is at $2 \mathrm{~km}$. Horizontal axis is linear in offset, but labeled in equivalent angle of incidence. (a) data for the $\mathrm{X}$-source into the $\mathrm{X}$ receiver $(\mathrm{X}-\mathrm{X})$; (b) $\mathrm{Y}$-source $\mathrm{X}$ receiver; (c) X-source, Y-receiver; (d) Y-source, Y-receiver. Note that in the purely isotropic model, there is no energy on the $\mathrm{Y}-\mathrm{X}$ or $\mathrm{X}-\mathrm{Y}$ components. Also note the significant difference in reflection amplitudes with angle of incidence in the X-X (SV) and Y-Y (SH) components. 145

Figure 57. A: Correction for SV and SH amplitude applied to synthetic data from Figure 57 for an X-oriented time-distance profile with amplitude correction. Note the two layer isotropic model outlined in Table 1. Layer depth is at $2 \mathrm{~km}$. Horizontal axis is linear in offset, but labeled in equivalent angle of incidence. (a) data for the $\mathrm{X}$-source into the $\mathrm{X}$ receiver (X-X); (b) Y-source X receiver; (c) X-source, Y-receiver; (d) Y-source, Y-receiver. Note the nearly constant amplitude of the XX and YY panels since we tried to remove the AVO effect from the polarization distortion. .146 
Figure58: Time-distance (offset) synthetic profile oriented at a $30^{\circ}$ to the $\mathrm{X}$ axis for a two layer isotropic model outlined in Table 1. Layer depth is at $2 \mathrm{~km}$. Horizontal axis is linear in offset, but labeled in equivalent angle of incidence. (a) data for the $\mathrm{X}$-source into the $\mathrm{X}$ receiver $(\mathrm{X}-\mathrm{X})$; (b) $\mathrm{Y}$ source X receiver; (c) X-source, Y-receiver; (d) Y-source, Y-receiver. X and $\mathrm{Y}$ receiver source showing a source-receiver azimuth of $30^{\circ}$. Note the cross term energy in the (XY) and (YY) resulting solely from difference in SV-SV and SH-SH reflectivity and the lack of alignment between the source-receiver azimuth and the orientation of the horizontal source and receivers. 148

Figure 58. A: Polarization correction and rotation of $30^{\circ}$ oriented profile (Figure 58) to an X-oriented profile applied to data in Figure 58. The polarization correction removes the AVO different effect on the SV and SH. Layer depth is at $2 \mathrm{~km}$. Horizontal axis is linear in offset, but labeled in equivalent angle of incidence. (a) data for the $\mathrm{X}$-source into the $\mathrm{X}$ receiver (X-X); (b) Y-source X receiver; (c) X-source, Y-receiver; (d) $\mathrm{Y}$-source, Y-receiver. The result is the offset-corrected Alford rotation angle of $30^{\circ}$. Note the removal of the cross term energy in the (XY) and (YY) resulting solely from difference in SV-SV and SH-SH reflectivity (compare to Figure 57A). There is some residual cross-term energy present, but it is so minimal that it appears that all the energy is removed. 149 
Figure 59: Synthetic data from the two layer models isotropic/ anisotropic model outlined in Table 5. The layer depth at $2 \mathrm{~km}$. Horizontal axis is linear in offset, but labeled in equivalent angle of incidence. (a) data for the $\mathrm{X}$ source into the $\mathrm{X}$ receiver $(\mathrm{X}-\mathrm{X})$; (b) $\mathrm{Y}$-source $\mathrm{X}$ receiver; (c) $\mathrm{X}$-source, Y-receiver; (d) Y-source, Y-receiver. Note zero crossing occurs at $22^{\circ}$ for $\mathrm{X}-\mathrm{X}$ and $50^{\circ}$ for $\mathrm{Y}-\mathrm{Y}$ panels with cross-term energy being present indicating anisotropy. The zero crossing on the Y-Y panel is difficult to see because of out of plane energy being present. Note the change in trace amplitude from $0^{\circ}$ to $50^{\circ}$. No polarization correction rotations have been applied. 153

Figure 60: Polarization correction applied to synthetic data (Figure 59) from the two layer model isotropic/ anisotropic model after polarization correction. The layer depth at $2 \mathrm{~km}$. Horizontal axis is linear in offset, but labeled in equivalent angle of incidence. (a) data for the $\mathrm{X}$-source into the $\mathrm{X}$ receiver $(\mathrm{X}-\mathrm{X})$; (b) Y-source $\mathrm{X}$ receiver; (c) X-source, Y-receiver; (d) Y-source, Y-receiver. Note constant amplitude of X-X and Y-Y panels and how the cross-term energy changes after polarization correction has been applied. Also the zero crossing $\left(50^{\circ}\right)$ on the $\mathrm{Y}-\mathrm{Y}$ synthetic seismic but other traces are also muted due to the high amplitude nature after polarization correction is applied 154 
Figure 61: Diagram shows Alford rotation angle scan from $0^{\circ}$ to $90^{\circ}$ in ten degree increments to determine what angle causes the cross-term energy to disappear. The rotation angle projects these data to SV and $\mathrm{SH}$ coordinates consistent with the HTI geometry, and the cross-term energy is minimized. Note that we still see the presence of energy on the crossterms because the fracture strike direction is $30^{\circ}$. The original model has a $30^{\circ}$ fracture strike direction and the plot indicates that at $30^{\circ}$ the crossterm energy is zero and the $\mathrm{X}-\mathrm{X}$ and $\mathrm{Y}-\mathrm{Y}$ receiver pairs are maximum. As noted all other rotation angles the cross-term energy is not zero meaning it's not at the correct orientation. The data is collected over a two layer models isotropic/ anisotropic model outlined in Table 5 after polarization correction. The layer depth at $2 \mathrm{~km}$. 155

Figure 62: Original field data showing $\mathrm{X}$ and $\mathrm{Y}$ source $-\mathrm{X}$ and $\mathrm{Y}$ receiver and zone of interpreted horizon (highlighted in yellow) is at $1100 \mathrm{msec}$. Note the noisy nature of the field data before the polarization correction and Alford rotations have been applied. 160

Figure 63: Polarization correction applied to the original field data showing $\mathrm{X}$ and $\mathrm{Y}$ source $-\mathrm{X}$ and $\mathrm{Y}$ receiver and zone of interpreted horizon is at 1100 msec. Note the major difference in the continuity of the beds and the amplitudes, between the SxRx, SyRy and the SxRy and SyRx data. No NMO correction applied. 161 
Figure 64: Polarization correction is applied to the seismic panels (Figure 63) and an Alford rotation azimuth scan of $10^{\circ}$. Note there is no major difference in bed continuity and amplitudes. The data is very noisy partly due to the high levels of azimuthal anisotropy that dominates the reflected $\mathrm{S}$ waves.

Figure 65: Polarization correction is applied to the seismic panels and an Alford rotation azimuth scan of $30^{\circ}$. Note there is no difference in the continuity of the beds and the amplitudes. The data is very noisy due to the high levels of azimuthal anisotropy that dominates the reflected $\mathrm{S}$ waves. All the azimuth scans look very noisy due to the high degree of anisotropy in the shallow section. 163

Figure 66: Polarization correction is applied to the seismic panels and an Alford rotation azimuth scan of $70^{\circ}$. Note the major difference in the continuity of the beds and the amplitudes. The data is very noisy due to the high levels of azimuthal anisotropy that dominates the reflected S waves. 164

Figure 67: Scan of field data with $\mathrm{X}$ (east) and Y (north) sources with $\mathrm{X}$ (east) and $\mathrm{Y}$ (north) receivers, in the original field coordinates after applying the offset corrected Alford's rotation and polarization correction to all the data. Rotations have been applied using $\theta$ in ten degree increments. Correction is based on reflection geometry to the $1100 \mathrm{msec}$ reflector. There is energy on the cross-terms which is indicative of anisotropy. The values of gamma are so large that they do affect the reflection process. Cross-term energy is minimum at $30^{\circ}$ and $\mathrm{X}-\mathrm{X}$ and $\mathrm{Y}-\mathrm{Y}$ receiver pairs are maximum, which is the fracture strike at this depth. Note all other rotation angles the cross-term energy is present indicate anisotropy.165 xlix 


\section{CHAPTER 1}

\section{HYPOTHESIS, INTRODUCTION AND BACKGROUND}

The objective of this study is to fully describe, experimentally confirm and document the predicted distortion of polarization in seismic shear-wave reflections in isotropic and anisotropic materials and to develop strategies to correct for this distortion. The basic hypotheses of this study are a) Seismic shear waves undergo significant distortion in polarization upon reflection (even in purely isotropic media) and b) This distortion can be corrected with sufficient precision to allow interpretation of polarization information to infer bulk elastic properties of the propagating medium to which polarization of seismic shear waves are sensitive. This requires determining the factors that influence propagation of shear waves and understanding how these factors affect characterization and evaluation of subsurface reservoir properties by analysis of S-wave data, particularly polarization information. Azimuthal Anisotropy associated with vertical fracturing is one of the primary polarization properties that influence such shear-wave propagation. This investigation explores the variations and characterization of this distortion in polarization, its characteristics in both isotropic and anisotropic media and how effectively we can correct for this polarization distortion.

\section{Seismic Waves}

Seismic $\mathrm{P}$ waves and $\mathrm{S}$ waves are two types of seismic body waves that propagate through solid materials (Sheriff and Geldart, 1995, see figure 1). Propagation of primary (P) waves is illustrated in Figure 1 and propagation velocity is described by equation 1 for homogeneous and isotropic media, also known as compressional waves or P-waves, primary waves respond to two elastic parameters (incompressibility and rigidity) and 
mass density, with particle motion polarized in a direction parallel to the direction of propagation (Tatham and McCormack, 1991).

$$
\begin{gathered}
P\left(\text { Primary)wave Velocity }=\sqrt{\frac{\mathrm{k}+4 / 3 \mu}{\rho}}\right. \\
S\left(\text { Shear)wave velocity }=\sqrt{\frac{\mu}{\rho}}\right.
\end{gathered}
$$

where

$\mathrm{k}$ - bulk modulus

$\mu$ - shear modulus

$\rho$ - density

Shear wave propagation is illustrated in Figure $1 \mathrm{~b}$ and propagation velocity is described by equation 2 for homogeneous and isotropic media. Unlike P-waves however, shear waves respond only to rigidity (shear modulus) and density, with particle motion polarized normal to the direction of wave propagation (Tatham and McCormack, 1991). This transverse polarization orthogonal to the direction of propagation may take on may directions that is orthogonal to the propagation direction and is often described in terms of two orthogonal basis components describing shear-wave polarization related to a vertical plane connecting the source and receiver positions. These two basis components are commonly referred to as $\mathrm{SH}$ and $\mathrm{SV}$, denoting polarization normal to the vertical plane ( $\mathrm{SH}$ ) defined by the source and receiver locations, and particle motion within the vertical plane (SV) for an isotropic medium (Figure 2). It is important to note that nearly all descriptions and analysis of reflections, transmission and mode-conversion processes are ultimately described in terms of the individual SV and SH components. Also note that the SH polarization, normal to the vertical plane, is always horizontal. The SV 
polarization or particle motion entirely within the vertical plane is vertical only for a horizontal propagation direction.

Ultimately, in anisotropic media, the propagating shear waves become polarized in the natural symmetry axes of anisotropy of the propagating medium (Gumble, 2006). In a transversely isotropic medium, a commonly assumed symmetry of anisotropy used to describe S-wave propagation in a simple anisotropic medium, within shear waves propagating in a direction normal to the symmetry axis (parallel to the 'plates'), a fast shear component (S1) is polarized in the direction parallel to the "plates" will be the higher propagation velocity of the shear-wave polarizations in medium, whereas the slower shear component (S2) with the same propagation direction as S1, will be polarized orthogonal to the S1 direction, but normal to the plates (figure 3). The differences in the propagation velocity of these two preferred S-wave polarizations are commonly referred to as birefringence. The ray path geometry of the S1 and S2 components are identical. The velocity difference between the S1 and S2 components may be used as a measure of the magnitude of the anisotropy of the shear-wave medium (Tatham et al., 1992). 


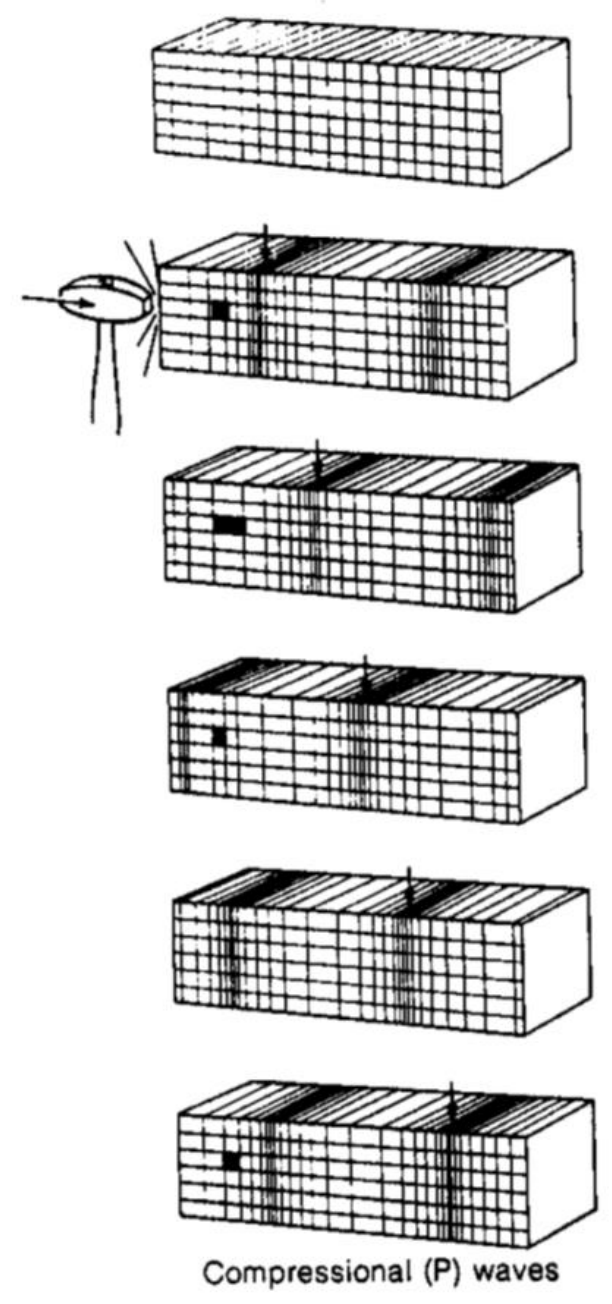

(a)
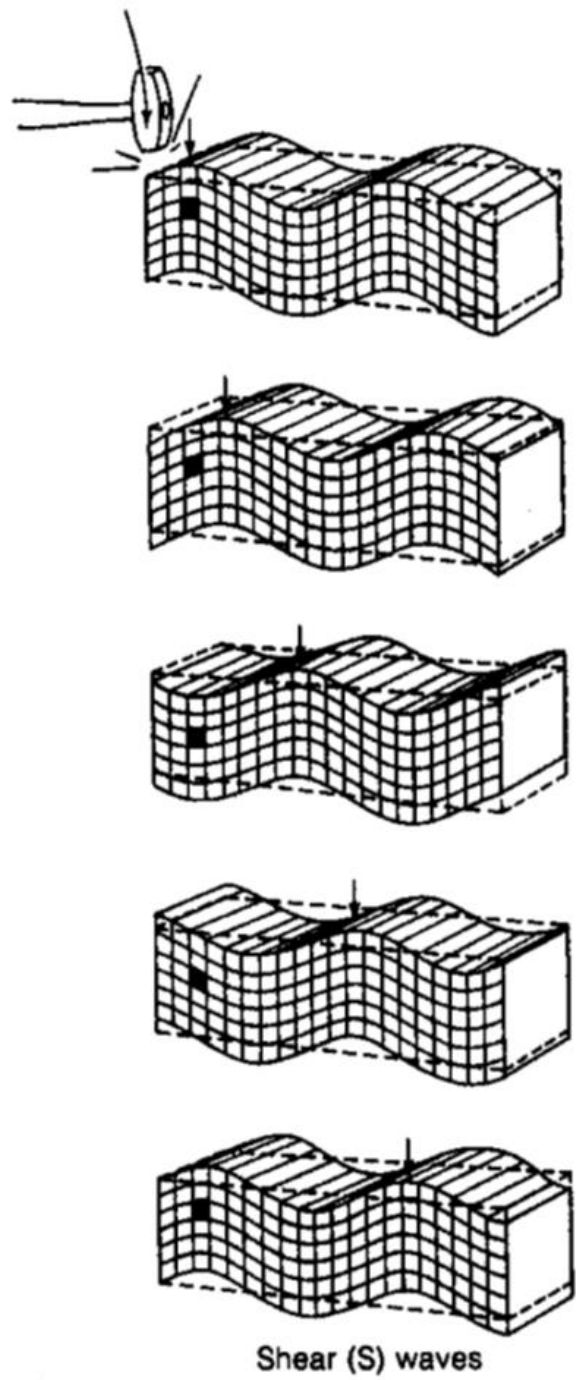

(b)

Figure 1: Particle motion during horizontal passage of plane body waves, (a) P-waves with particle motion parallel to the propagation direction and (b) S-waves when polarization is perpendicular to the propagation direction. Note that the particle motion of the propagating P-wave is in the direction of propagation so polarity is defined solely by the propagation direction. The particle motion of the propagating S-wave is orthogonal to the direction of propagation, so there is a wide variety, $\left(360^{\circ}\right)$ of polarizations for a given propagation direction (Sheriff and Geldart, 1993). 


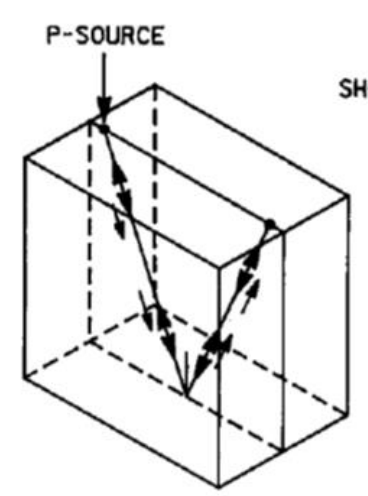

(a)

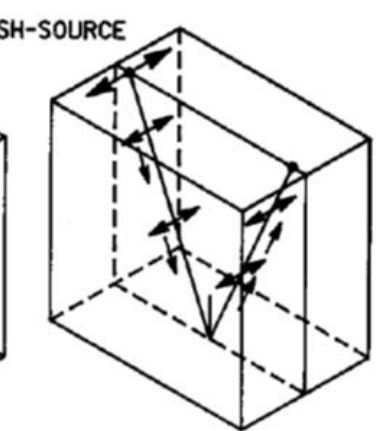

(b)

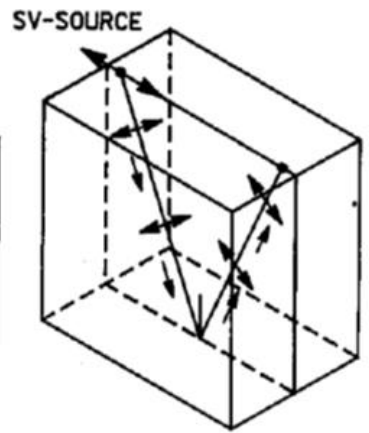

(c)

Figure 2: Ray propagation and polarization directions, with propagation shown by smaller arrows next to rays, and particle motion shown by two-headed arrows for (a) P-P, (b) SH-SH and (c) SV-SV waves (Tatham and McCormack, 1991). Note that P-wave particle motion is parallel to the propagation direction and in the vertical plane defined by the source and receiver position. SV particle motion is normal to the propagation direction and in the vertical plane. SH particle motion is normal to the propagation direction and normal to the vertical plane. The particle motion of the $\mathrm{SH}$ wave is always horizontal but the particle motion of SV wave, although in the vertical plane, is not, in general, horizontal. In general, a propagating shear wave will have an arbitrary polarization normal (transverse) to the propagation direction. For most calculations, the physics suggest that we generally address any shear-wave polarization in terms of orthogonal SV and SH components. 


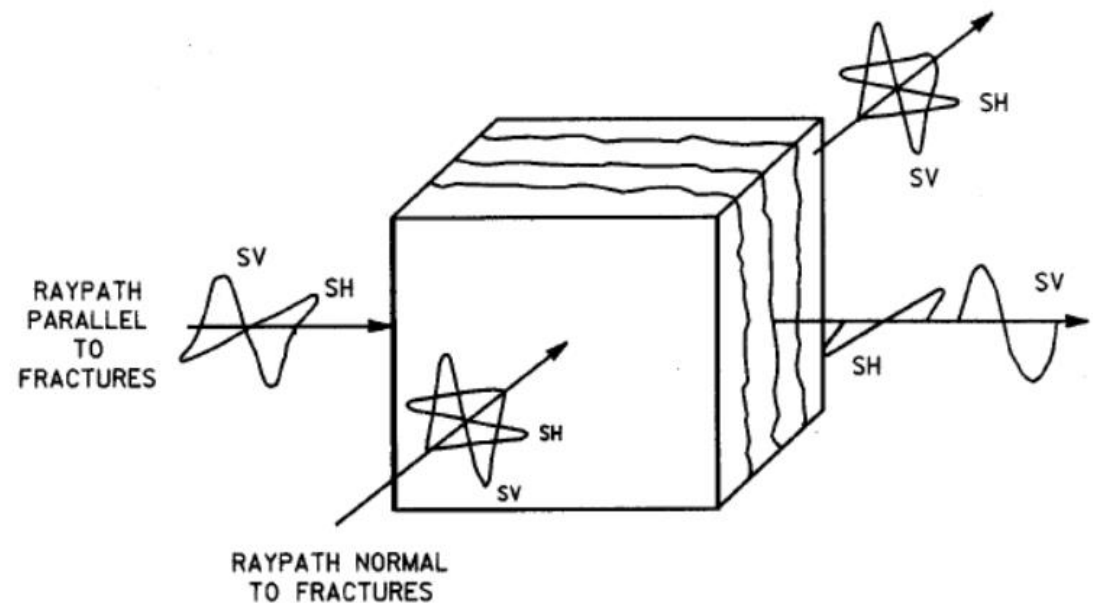

Figure 3: The effect of HTI anisotropy possible association with vertical fractures, on horizontal SV and SH wave- propagation. Note the relationship between fracture orientation and polarization of S-waves exiting the medium (Tatham and McCormack, 1991). In this example for a horizontal propagation direction, the velocity of the $\mathrm{SH}$ wave (S2, with particle motion across the fractures) is less than the velocity of the SV wave with the particle motion parallel to the fractures, S1. The faster wave (SV fir horizontal propagation) is referred to as $\mathrm{S} 1$, while the slower wave is referred to as $\mathrm{S} 2$.

\section{Acquisition of Direct Seismic Shear-Wave Data in 3D}

For 2D acquisition of shear-wave data along a line (profile), the orientation of the direct horizontal shear-wave sources and receivers are commonly aligned 1) horizontally and parallel to the line direction and 2) horizontal, but normal to the line, or profile, direction. Thus, the source polarizations are, in principle, 1) in the vertical plane and 2) normal to the vertical plane as defined by any source and receiver pair (see figure 2). Hence the acquisition geometry for both the source and receivers is consistent with the orthogonal SV and SH basis components often used to describe shear-wave polarization. 
The same polarization correction is also commonly used for the horizontal receiver orientations.

For 3D acquisition, the sources and receivers are generally located on an areal surface and the individual source and receiver positions may be described in terms of $x-y$ Cartesian coordinates on the surface. The $\mathrm{x}$ and $\mathrm{y}$ coordinates may correspond to $\mathrm{X}$ and $\mathrm{Y}$, east and north geographical coordinates, respectively; oriented with respect to geological parameters such as regional strike and dip, or other survey parameters such as lease block descriptions. Here, I consider these $\mathrm{x}-\mathrm{y}$ coordinates as acquisition survey coordinates - controlled entirely by the survey design and independent of any geologic or geographic properties. Further, the polarizations of the direct shear sources and receivers are commonly oriented in the $\mathrm{x}$ and $\mathrm{y}$ survey axis directions. Significantly, the direction connecting any particular pair of source and receiver positions will not, in general, be oriented in either the $\mathrm{x}$ or $\mathrm{y}$ direction. This leads to orientation of each individual source and receiver not necessarily being aligned with the vertical plane connecting the source and receiver positions (see Figure 4). Each individual $\mathrm{x}$ and y source component is then recorded by both the $\mathrm{x}$ and $\mathrm{y}$ components of each receivers leading to four traces (Xsource, $\mathrm{X}$ receiver; $\mathrm{Y}$-source, $\mathrm{X}$ receiver; $\mathrm{X}$-source, $\mathrm{Y}$ receiver; $\mathrm{Y}$-source, $\mathrm{Y}$ receiver) associated with each source-receiver pair on the surface. In Figure 4, when the shear source is placed at the origin in the center of a field of receivers. Source polarization (direction of particle motion) is at the angle $\Psi$ with respect to $X$. Other quantities in Figure 4 of interest include the direction from the source position to any given receiver (source-receiver azimuth) $\theta$, and the orientation of the particle motion at the receiver (reflection polarization) position.

To fully analyze the reflection process between a single arbitrary source and receiver pair, each $\mathrm{x}$ and $\mathrm{y}$ shear source component and receiver component must be 
trigonometrically resolved into SV (parallel to the source-receiver direction) and $\mathrm{SH}$ (transverse to the source-receiver direction) component axes relative to the sourcereceiver azimuth. Once the polarization and amplitude information is resolved into SV and SH components further analysis of reflection amplitude and effective polarization may be applied. A basic thesis of this study is that, in general, the polarization of the recorded shear-wave reflection will not, in general, be the same as the original source polarization-even in purely isotropic materials. That is, the polarization of the shear wave is distorted as part of the reflection process.

Historically, the use of two dimensional profile recording, SH and SV source and receiver components have been directly recorded and no further trigonometric manipulation has necessarily been with the advent of densely sampled 3D data with aerially distributed source and receiver positions over a large area, data are not directly necessarily recorded in their direct $\mathrm{SV}$ and $\mathrm{SH}$ components. Thus three-dimensional seismic recording has led to additional complexities in the geometry and associated complexities in the meaning of the polarizations of the reflections.

The horizontal line between the source and receiver position on the surface defines the vertical plane that includes the radial particle motion. Waves detected in this geometry include both SV waves and P-waves, whereas the cross-line, or transverse component, contains only $\mathrm{SH}$ waves in an isotropic flat-layered earth. SH data are relatively simple in that there is no mode-conversion and other wave types. Propagating $\mathrm{SV}$ waves on the other hand are coupled with $\mathrm{P}$ waves which both have particle motion within the vertical plane. This leads to mode conversion between the two wave types as part of the reflection, transmission, mode-conversion process.

The mode conversion process happens when an incident $\mathrm{P}$-wave is reflected as SV, and vice versa (Tatham and McCormack, 1991). The P and SV particle motion are in 
the same vertical plane, while the $\mathrm{SH}$ is out of the plane. The $\mathrm{P}$ and $\mathrm{SV}$ motion being contained in the same plane results in coupling between the two waves, and their mutual effect on one another's reflection strength (Tatham and McCormack, 1991, p. 20). This is why, even though it is all shear motion, the two S-wave components may have significantly different reflection responses at an interface. Data with a compressional source and multicomponent receivers will record converted waves, where P-waves convert into SV -waves upon reflection or transmission (Shearer, 1999, p. 100). This will not occur for SH-waves in an isotropic medium, when the only source of SH-waves is a shear source (Shearer, 1999, p. 97). When an SH-wave is incident upon an interface, only two waves leave: a reflected SH-wave and a transmitted SH-wave (Shearer, 1999, p. 100). When an SV -wave is incident on the same interface four waves leave: a reflected and transmitted P-wave, and a reflected and transmitted SV -wave (Shearer, 1999, p. 100). Note that mode conversion does not occur at zero-offset, but the mode conversion affects the SV amplitude at small angles (Tatham and McCormack, 1991, p. 20). Further, the mode conversion affects the reflection strength (amplitude) of both the $\mathrm{P}$ and SV wave reflections. 


\begin{tabular}{|c|c|c|c|}
\hline & P-velocity $(\mathrm{km} / \mathrm{sec})$ & S-velocity $(\mathrm{km} / \mathrm{sec})$ & Density $(\mathrm{g} / \mathrm{cc})$ \\
\hline Layer 1 & 3.0 & 1.5 & 2.0 \\
\hline Layer 2 & 4.0 & 2.0 & 2.2 \\
\hline
\end{tabular}

Table 1: Two layer isotropic model with elastic and seismic properties.

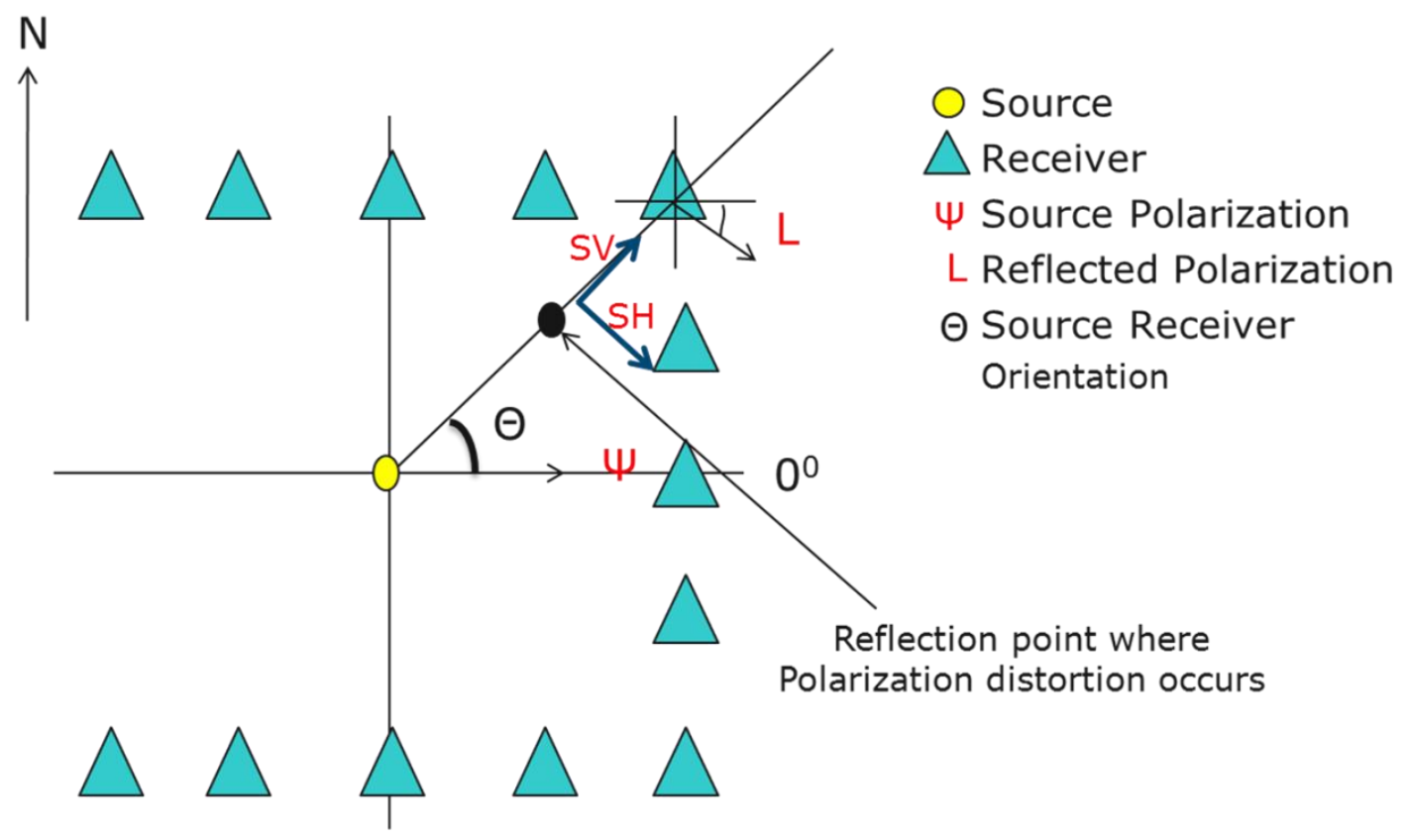

Figure 4: The acquisition geometry of a single source position in a 3D direct shear survey with a source polarization of $0^{\circ}$ (in general, a single horizontal shear source can have any polarization most direct-source 3D surveys utilizes two independent source polarizations, $\mathrm{X}$ and $\mathrm{Y}$ ) $\mathrm{L}$ is the observed polarization of the reflected wave is calculated. Note the significant derivation of the reflected shear-wave from the original source polarization (modified from Lyons, 2006). 


\section{Uses of Seismic Shear Waves}

Seismic shear waves with their transverse polarization are particularly useful in investigating the anisotropy of elastic media they propagate through; specifically for their sensitivity of velocities to variations in polarization direction for a single propagation path. This sensitivity provides information about the orientation of the fast and slow polarization direction and ultimately to the anisotropic parameters of the medium they propagate through. For such polarization information to be meaningful, however, there can be no distortion of polarization resulting from the reflection process itself. As discussed earlier, in analyzing shear-wave reflectivity, a general description of polarization of the incoming shear wave is typically defined in terms of two trigonometrically resolved orthogonal components ( $\mathrm{SV}$ and $\mathrm{SH}$ ) oriented with respect to the source and receiver locations on the surface. For normal incidence in isotropic media, SV and SH reflectivity is the same.

For non-normal angles of incidence, the reflectivity of the individual SV and SH components, computed from the well understood Zoeppritz (1919) equations, can be quite different (Figure 5). This difference in reflectivity amplitudes for these two components leads to significant change in the resultant polarization of an arbitrarily polarized reflected shear-wave relative to the incident wave. The SV wave changes polarity at an incidence angle of about $20^{\circ}$ and changes curvature shortly thereafter. The SV reflectivity has another change in curvature at about $30^{\circ}$, before it encounters an asymptote at around $47^{\circ}$ (which affects the SH reflectivity as well). The first change in curvature (first critical angle) corresponds to the P-wave refraction in the lower layer (Yilmaz, 2001, p. 1811). The second and third critical angles correspond to P-wave refraction in the upper layer (shear wave window), and S-wave refraction in the lower 
layer, respectively (Yilmaz, 2001, p. 1811). Note that, in this curve, the zero-crossing (zero amplitude for the SV-SV reflection) is at an incident angle less than any of the critical angles. The SH reflectivity changes very slowly, out to its zero-crossing at an incidence angle of about $40^{\circ}$.

Figure 5 also shows the phase changes in the SV-SV and SH-SH reflectivity, which may lead to changes in the reflected wavelet polarization $\left(180^{\circ}\right)$ and even the wavelet character for reflection angles beyond the first critical angle (see shear-wave window (Crampin, et al 1990). This shear-wave window effect may also lead to phase distortion of recorded shear-waves at large angles (beyond the first critical angle) or on free-surface of the earth beyond the first critical angle. The computed reflectivity relations illustrated in Figure 5 - variation in the reflection coefficient with angle of incidence - are defined by the Zoeppritz equations contrasts in elastic properties across reflecting interfaces (Table 1). Analysis of these variations in reflection strength with angle is particularly useful in conventional $\mathrm{P}$-wave reflectivity where the character of the amplitude vs. incidence angle (AVA) or, alternatively, AVO (amplitude vs. sourcereceiver offset) curve is used to isolate anomalous contrasts in Poisson's ratio (considered by many as a proxy for gas saturation). In conventional $\mathrm{P}$-wave data, the polarization (particle motion) of the propagating wave is the same as the propagation direction, so no additional information is available from analysis of polarization. This is not true of the transversely polarized shear wave. As seen in Figure 5, the reflection amplitudes for orthogonal SV and SH components are significantly different. In particularly the locations of zero-crossing points in the computed reflectivity curves are obvious parameters to observe. To consider the full reflectivity of an arbitrarily polarized shear wave, the observed shear wave must be geometrically described in terms of SV and SH components, the reflectivity computed separately for each component, and then 
recombined to estimate the polarization of the reflected wave. The differences in the SV and $\mathrm{SH}$ curves lead to differences in polarization according to the weighting between these two curves. This difference in weighting is the SV and SH components leads to the polarity distortion upon reflection of transversely polarized shear waves.

The phase differences between SV-SV and SH-SH reflections shown in Figure 5 may lead to more subtle effects than the amplitudes. For angles smaller than the first critical angle (SV-P mode converted reflection into the second layer), there is a $180^{\circ}$ phase reversal in the SV-SV reflection, but no phase distortion in the reflected wavelet. There is no mode-conversion associated with the $\mathrm{SH}-\mathrm{SH}$ reflectivity, so no critical angle associated with the much higher-velocity P-waves. The only critical angle for $\mathrm{SH}-\mathrm{SH}$ reflectivity occurs beyond the zero-crossing for the $\mathrm{SH}-\mathrm{SH}$ reflection and well beyond angles of incidence we are considering. Thus no phase distortion is associated with the $\mathrm{SH}-\mathrm{SH}$ reflection. Thus, prior to the SV-SV zero-crossing, there is no phase distortion in either the SV-SV or SH-SH reflection. Beyond the first critical angle, the phase distortion in the SV-SV is modest, (less than about $45^{\circ}$ ). Beyond the second critical angle (SV-P mode-conversion the internal refraction is in the upper layer) the SV-SV phase distortion in this range. Beyond the third critical angle for SV-SV (the first critical angle for SH$\mathrm{SH})$ the phase distortion is very pronounced.

\section{Optical Analogue}

This amplitudes distortion occurs even in isotropic media. This distortion

corresponds to a similar occurrence in optics, where Brewster's (polarizing) (Figure 6) angle named after the Scottish physicist, Sir David Brewster (1781-1868) (Hoenig and Moebius 1991) has no reflectivity in the equivalent SV component, and all the reflected 
energy has solely SH polarization-completely independent of the incoming polarization. Thus, this incidence angle is commonly termed the polarization angle in optics.

The "elastic analogue" to Brewster's angle of optics is more complex in the elastic case in that the incoming SV wave can also be reflected and refracted as a modeconverted $\mathrm{P}$ wave. Although $\mathrm{SV}$ and $\mathrm{SH}$ reflections exhibit a similar transverse polarization as plane polarized light at a plane interface, the physics of elastic wave propagation is very different. A simple description would be that, in optics this behavior is governed by the mixing between the differently, but still transversely polarized light waves. In elasticity this behavior includes coupling of SV polarized shear waves with the P-waves, and the generation of entirely new mode-converted P-waves. Further, there is no SV-P coupling to the SH polarized shear wave in isotopic media. Enhancing the difference in reflectivity between the SV and SH components. Basically, there is no Pwave analogue in optics. In the simplest, elastic case of purely isotropic media, for a horizontal reflecting interfaces $\mathrm{P}$ and SV waves are coupled and that SH waves stand alone and are not coupled to P and SV waves.

Interestingly, analysis of the reflection phenomena of incident seismic SV waves also shows that $\mathrm{SV}$ reflectivity vanishes at a relatively small range $\left(\sim 20^{\circ}-25^{\circ}\right)$ of incidence angles for a wide range in contrasts of seismic properties (Krohn, 1988; Gumble, 2006; Lyons, 2006; Campbell and Tatham 2011). The apparent narrow range of this angle independent of the constants in the elastic properties suggests that a correction based on zero crossing values for this effect may potentially be relatively insensitive to precise knowledge of the actual values of velocities and densities across the reflecting interface. On the other hand, the rapid change in reflection amplitude with respect to incidence angle at the zero crossing will lead to strong sensitivity to errors in estimation of the zero-crossing. Thus a "notch" mute in the corrections may be required around the 
zero-crossing angle. The SV and SH curves do show interesting amplitude character with angle of incidence (AVA) and may be used for analysis in themselves. To perform this type of analysis requires that the observed shear-wave polarizations be transformed to SV and $\mathrm{SH}$ components - completely forsaking any polarization information included in the data. As discussed later, any proposed amplitude correction for polarization distortion will require removing the amplitude effects of variations with angle of incidence (offset) to preserve the polarization information. This analysis of reflection amplitudes with offset variations and observed polarization will require entirely separate and independent analysis tracks.

Simmons (2004) identified the presence of what he described as "cross-term" energy (even in isotropic media) when simulating or recording a 3D survey utilizing direct shear-wave sources and receivers. This results from same variations in SV-SV and SH-SH energy considered here being projected onto the receiver components due to generalized 3D acquisition geometry and differences in SV and SH reflectivity and possibly heterogeneity. Our investigation expands on that work and explores issues related to polarization analysis, and how to decompose the record polarization information.

The analysis discussed by Simmons (2004) is focused on reducing the amplitude vs. offset considered to SH-SH reflectivity and thus no consideration of wavelet phase distortion is necessary. For the present analysis, the wavelet phase distortion is limited to the reflections beyond the first SV-SV critical angle, which is beyond the SV-SV zero crossing. In practice, I expect the proposed polarization corrections to be most important at angles up to the SV-SV zero crossing where there is no wavelet distortion. Thus, consideration of the potential phase distortion is deferred to expansion of the current work to larger angles of incidence. 

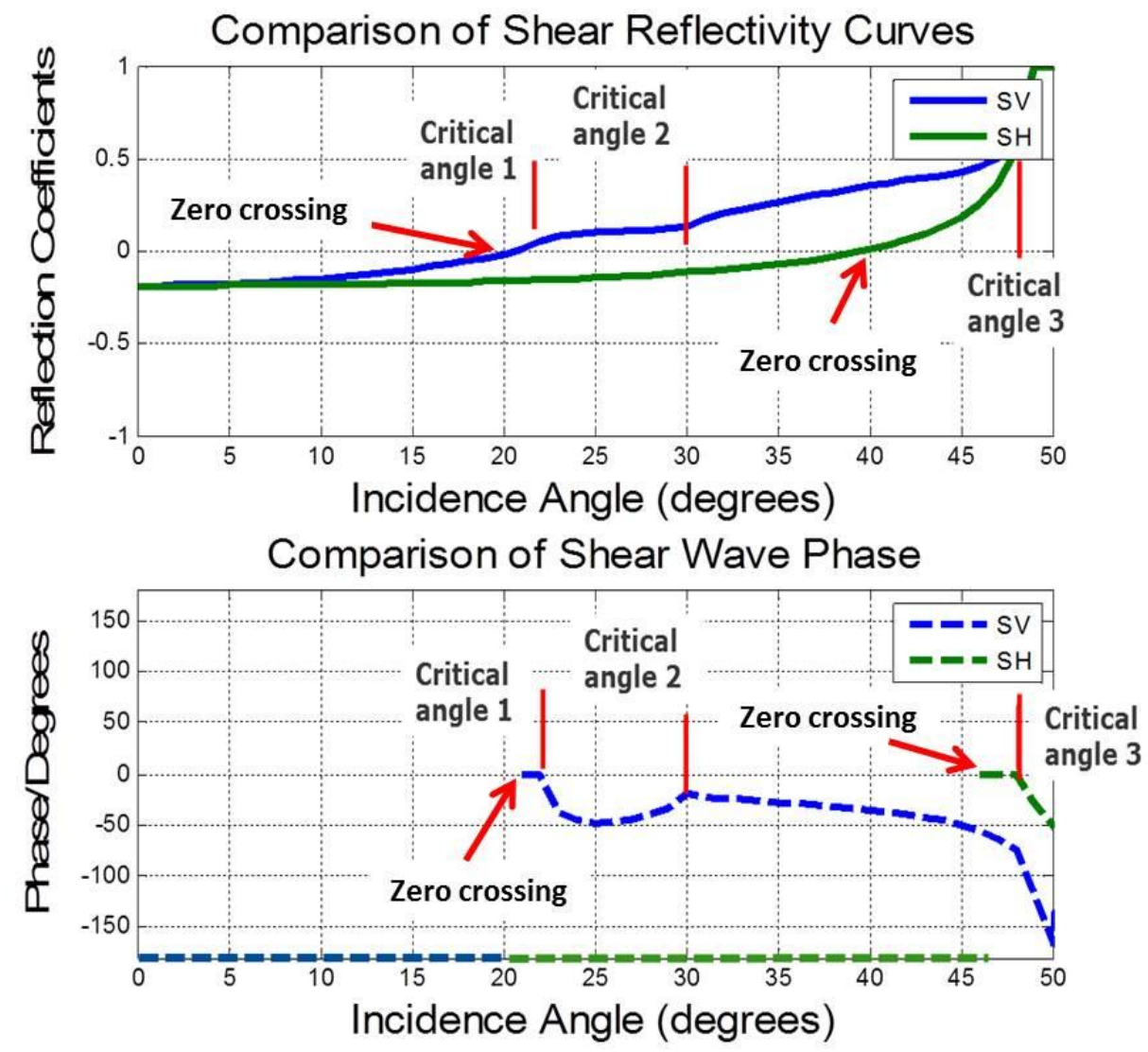

Figure 5: Reflection coefficients for SV-SV (blue) and SH-SH (red) waves showing that the reflection coefficients vanish at some particular angle of incidence. Velocity and density information for all the examples shown is given in table 1.The position of the three critical incidence angles are shown. The three critical angles relate to the P-wave mode-converted and refracted wave into the lower layer $\left(22^{\circ}\right)$, the internally refracted mode-converted $\mathrm{P}$-wave in the upper medium $\left(30^{\circ}\right)$ and the refracted S-wave in the lower layer $\left(49^{\circ}\right)$. For this set of elastic and density parameters zero crossing is at $20^{\circ}$ for $\mathrm{SV}$ and $40^{\circ}$ for $\mathrm{SH}$. Note the constant phase of both SV-SV and SH-SH reflections before the first critical angle. Beyond the first critical angle which is beyond the SV-SV zero-crossing, there is a modest phase change for the SV-SV reflection but no phase change for the $\mathrm{SH}-\mathrm{SH}$ reflection until the one critical angle for the SH wave. Beyond the second critical angle of the SV-SV reflection the phase changes again from 40 degrees to 180 degrees to the third critical angle. The magnitude is shown as positive or negative in order that the phase will always be zero below the first critical angle, and as continuous as possible beyond that. The phase is always zero or pi in this region. 


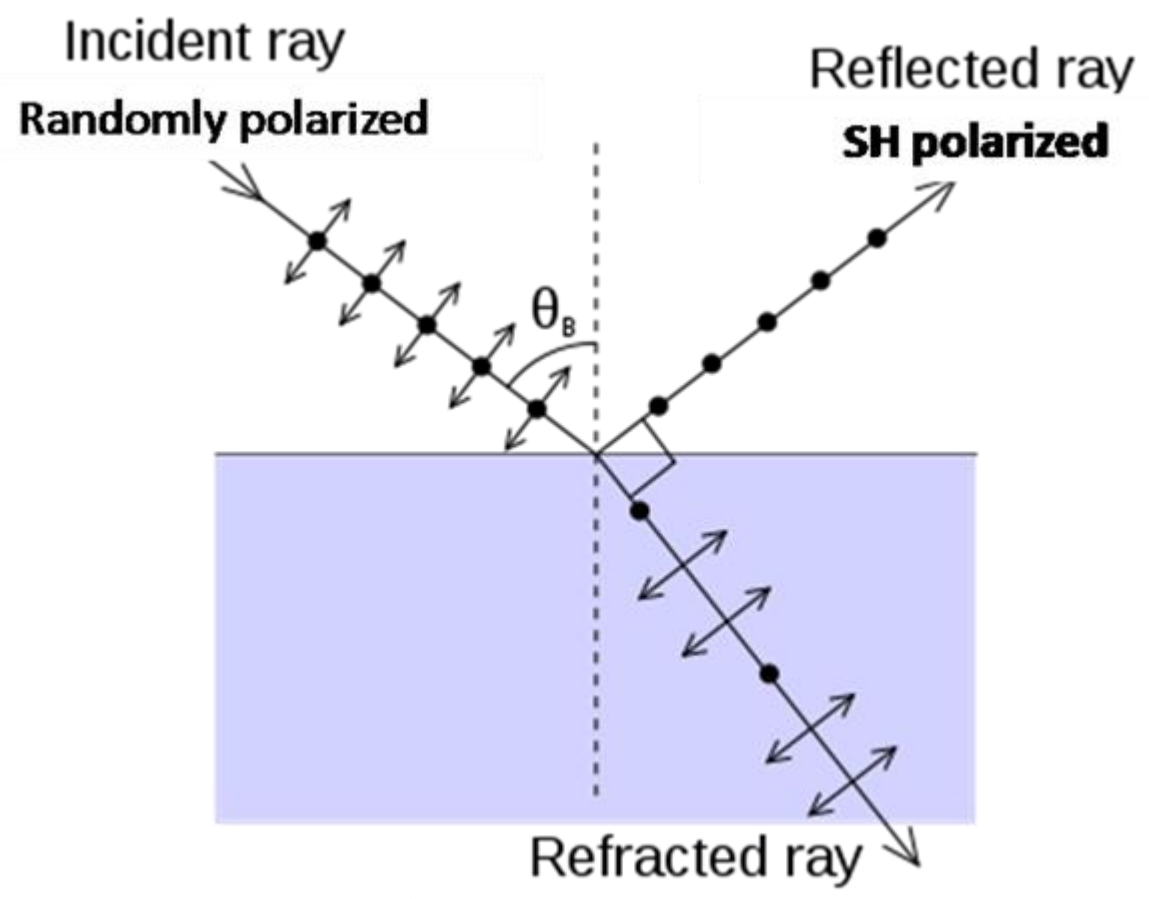

Figure 6: The optical Brewster's angle (also known as the polarization angle) is an angle of incidence at which transversely polarized light, which is transversely polarized, with a particular polarization (equivalent to the seismic SV wave) is only transmitted through a surface, with no reflection. Interestingly, the ray-direction of the reflected wave aligns with the orientation of the equivalent SV component of the incident ray. For this angle the reflected wave is only polarized as an SH-wave thus the term "polarized angle". This angle is named after the Scottish physicist, Sir David Brewster (1781-1868). In the elastic case the randomly polarized $\mathrm{S}$-wave is distorted upon reflection due to a deviation of reflection geometry from a purely $\mathrm{SV}$ and $\mathrm{SH}$ situation. In this example (as most optical examples), the lower medium has a lower velocity, the upper (air) medium. Thus, no critical angle exists and there is no phase distortion present. 


\section{Simulation of Polarization Distortion}

Note that all the seismic shear sources used for demonstrating the present discussion of polarization distortion are direct (generally horizontal) shear wave sources with defined horizontal polarities (Tatham and McCormack, 1991; Simmons, 1999). For analysis, the original input source polarizations are trigonometrically projected into SV and SH components for their specific source and receiver positions. For simulation purposes, the full Zoeppritz reflection coefficients (Aki and Richards, 2002) are calculated and applied to the individual incoming SV and SH components of the incoming S-wave. I first address SV-SV reflection, followed by much simpler SH-SH reflectivity, (with no mode conversion involved).

Zoeppritz (1919), developed a set of equations, and I apply them as they are commonly used today (Aki and Richards, 2002). For incident P and SV -waves, the computation method involves populating two matrices $\mathbf{M}$ and $\mathbf{N}$ with combinations of elastic parameters and incident angles:

$$
\boldsymbol{M}=\left(\begin{array}{cccc}
-\alpha_{1} p & -\operatorname{cosj}_{1} & \alpha_{2} p & \cos _{2} \\
\operatorname{cosi}_{1} & -\beta_{1} p & \operatorname{cosi}_{2} & -\beta_{2} p \\
2 \rho_{1} \beta_{1}{ }^{2} \operatorname{posi}_{1} & \rho_{1} \beta_{1}\left(1-2 \beta_{1}{ }^{2} p^{2}\right) & 2 \rho_{2} \beta_{2}{ }^{2} \operatorname{cosi}_{2} & \rho_{2} \beta_{2}\left(1-2 \beta_{2}{ }^{2} p^{2}\right) \\
-\rho_{1} \alpha_{1}\left(1-2 \beta_{1}{ }^{2} p^{2}\right) & 2 \rho_{1} \beta_{1}{ }^{2} p \operatorname{cosj}_{1} & \rho_{2} \alpha_{2}\left(1-2 \beta_{2}{ }^{2} p^{2}\right) & -2 \rho_{2} \beta_{2}{ }^{2} \operatorname{pcosj}_{2}
\end{array}\right)
$$

and

$$
\boldsymbol{N}=\left(\begin{array}{cccc}
\alpha_{1} \mathrm{p} & \operatorname{cosj}_{1} & -\alpha_{2} \mathrm{p} & -\cos _{2} \\
\operatorname{cosi}_{1} & -\beta_{1} \mathrm{p} & \operatorname{cosi}_{2} & -\beta_{2} \mathrm{p} \\
2 \rho_{1} \beta_{1}{ }^{2} \mathrm{pcosi}_{1} & \rho_{1} \beta_{1}\left(1-2 \beta_{1}{ }^{2} \mathrm{p}^{2}\right) & 2 \rho_{2} \beta_{2}{ }^{2} \operatorname{posi}_{2} & \rho_{2} \beta_{2}\left(1-2 \beta_{2}{ }^{2} \mathrm{p}^{2}\right) \\
\rho_{1} \alpha_{1}\left(1-2 \beta_{1}{ }^{2} \mathrm{p}^{2}\right) & -2 \rho_{1} \beta_{1}{ }^{2} \cos _{1} & -\rho_{2} \alpha_{2}\left(1-2 \beta_{2}{ }^{2} \mathrm{p}^{2}\right) & 2 \rho_{2} \beta_{2}{ }^{2} \operatorname{pcos}_{2}
\end{array}\right) \text { (4) }
$$


where:

$\alpha_{n}=\mathrm{P}$-wave velocity in medium

$\beta_{n}=\mathrm{S}$-wave velocity in medium

$\rho_{n}=$ density velocity in medium

for $n=1$ or 2 corresponding to the upper and lower medium.

$i_{n}=$ angle between the ray direction of $\mathrm{P}$-wave propagation and the vertical direction for the P-wave in medium n,

$j_{n}=$ angle between the ray direction of S-wave propagation and the vertical direction from the S-wave in the medium,

for $\mathrm{n}=1$ or 2 for the upper and lower medium

$\mathrm{p}=\frac{\operatorname{sini}_{n}}{\alpha_{n}}=\frac{\sin j_{n}}{\beta_{n}} \equiv$ "ray parameter"

which is an expression for Snell's law.

The various reflection, refraction and mode conversion coefficient can be obtained by multiplying the inverse matrix $\mathrm{M}$ with matrix $\mathrm{N}$ matrices (see equation 5). The parameters, in the nomenclature of Aki and Richards (2002) used are density ( $\rho)$, P-wave velocity $(\alpha)$, S-wave velocity $(\beta)$, incident angles (i for P waves, $\mathrm{j}$ for $\mathrm{S}$-waves), and $\mathrm{p}$, ray parameter which is defined as the sine of the incident angle divided by velocity of that wave type in the medium in question. The upper layer (containing the incident wave) parameters are denoted by a subscript of 1 , the lower layer by 2 .

This yields a matrix of reflection, conversion, and transmission coefficients,

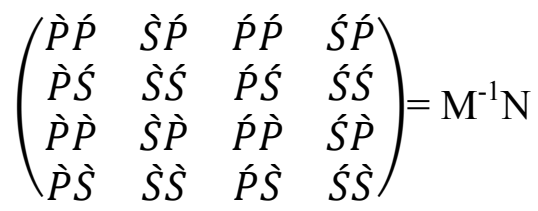


From this Aki and Richard (2002) isolate the downgoing incident SV-upgoing (reflected) SV wave $\grave{S} S$ (second column, second row of coefficient matrix), the $\grave{S} S$ of Aki and Richards (2002). The equation for coefficients of the downgoing SV and up-going SVwave may be represented as (Aki and Richards, 2002, p. 139):

$$
\grave{S} S=\left[\left(b \frac{\cos j_{1}}{\beta_{1}}-c \frac{\cos j_{2}}{\beta_{2}}\right) E-\left(a+d \frac{\cos i_{2}}{\alpha_{2}} \frac{\cos j_{1}}{\beta_{1}}\right) G p^{2}\right] / D
$$

where subscript 1 and 2 represent the upper and lower medium and the constants are:

$$
\begin{gathered}
\mathrm{a}=\rho_{2}\left(1-2 \beta_{2}^{2} \mathrm{p}^{2}\right)-\rho_{1}\left(1-2 \beta_{1}^{2} \mathrm{p}^{2}\right) \\
\mathrm{b}=\rho_{2}\left(1-2 \beta_{2}^{2} \mathrm{p}^{2}\right)+2 \rho_{1} \beta_{1}^{2} \mathrm{p}^{2} \\
\mathrm{c}=\rho_{1}\left(1-2 \beta_{1}^{2} \mathrm{p}^{2}\right)+2 \rho_{2} \beta_{2}^{2} \mathrm{p}^{2} \\
\mathrm{E}=\mathrm{b} \frac{\operatorname{cosi} \mathrm{i}_{1}}{\alpha_{1}}+\mathrm{c} \frac{\cos \mathrm{i}_{2}}{\alpha_{2}} \\
\mathrm{~F}=\mathrm{b} \frac{\cos \mathrm{j}_{1}}{\beta_{1}}+\mathrm{c} \frac{\cos \mathrm{j}_{2}}{\beta_{2}} \\
\mathrm{D}=\mathrm{EF}+\mathrm{GHp}=(\mathrm{det} \mathbf{M}) /\left(\alpha_{1} \alpha_{2} \beta_{1} \beta_{2}\right) \\
\mathrm{G}=\mathrm{a}-\mathrm{d} \frac{\operatorname{cosi} i_{1}}{\alpha_{1}} \frac{\cos \mathrm{j}_{2}}{\beta_{2}} \\
\alpha_{2}
\end{gathered}
$$


The $\mathrm{P}$ and $\mathrm{SV}$-wave coefficients are calculated together because they are mechanically coupled, which will be explained, not simply outlined in the section of this chapter on seismic basics.

The simpler equation for $\mathrm{SH}-\mathrm{SH}$ wave reflection is:

$$
\grave{S} \hat{S}=\frac{\rho_{1} \beta_{1} \cos j_{1}-\rho_{2} \beta_{2} \cos j_{2}}{\rho_{1} \beta_{1} \cos j_{1}+\rho_{2} \beta_{2} \cos j_{2}}
$$

(Aki and Richards, 2002, p. 139)

The results of these calculations for SV-SV and SH-SH are illustrates in Figure 5. In general, all the reflection coefficients are complex numbers but then only have imaginary components beyond the first critical angle (where the sine of a reflected angle exceeds unity). The fully complex nature of the coefficients is characterized by reducing the reflection coefficients to elements of amplitude and phase. The following calculations utilize only the amplitude element (corrected for a $180^{\circ}$ phase value) and thus do not include the phase and component of the phase component may be considered as wavelet distortion in the actual field elements.

For different source-receiver azimuths, the separate SV and SH reflection amplitude components are trigonometrically combined to define the resultant net amplitude and polarization of the reflected shear wave. The results for the reflection coefficients of the polarization distortions for a single shear source location with shear polarization oriented in the 'east' direction $\left(0^{\circ}\right.$ orientation $)$ and recorded over an areal plane are shown in Figure 7.

The length of the arrows shows the amplitude of the reflected wave and the orientation of the arrows shows the resultant polarization. In this plot, all polarizations on the $\mathrm{x}$ axis are all purely $\mathrm{SV}-\mathrm{SV}$, and on the $\mathrm{y}$ axis $\mathrm{SH}-\mathrm{SH}$. Note that there is no 
polarization distortion in these two directions (the polarization of the reflected wave is reversed, but not distorted, due to the $180^{\circ}$ phase reversal for normal incidence reflector). Also note that there is considerable amplitude variation (length of arrows) in these directions, following the pure SV-SV and SH-SH reflectivity curves. At receiver positions between the SV and SH orientations for this single source, the reflected polarization is strongly distorted up to $45^{\circ}$ at offsets of $3000 \mathrm{~m}$. The results for the reflection coefficients of the polarization distortions for a source oriented in the 'north' direction $\left(90^{\circ}\right.$ orientation) are shown in Figure 8. This simulation illustrates a source orthogonal to that in Figure 7 and completes a full set of horizontal source orientations. Note that this single source is just a $90^{\circ}$ rotation of that in Figure 7. Keep in mind that these two (X and Y) source polarizations are what would typically be used in an actual 3D shear source survey. In the observed polarization plot for the source oriented in the east direction (Figure 7), the SV-SV zero crossing phenomena are readily identifiable. We see the polarity change with offset at this distance, as well as purely SH reflectivity. The resultant reflection polarizations for separate $\mathrm{X}$ and $\mathrm{Y}$ horizontal oriented shear sources into an areal surface array of $\mathrm{X}$ and $\mathrm{Y}$ oriented receivers. Note the wide variation of the resultant reflection polarization of the shear-to-shear reflection for a single source polarization, even for this purely isotropic media. The SV zero crossings are located on either side of the SV source, at approximately 1800 meters offset true in any direction. The SV zero crossings, always occurs at a smaller distance (or angle) than the $\mathrm{SH}$ crossing.

These distortions are for a true impulse response, with no wavelet distortion considered. The SV nulls also serve as the focus points around which the observed polarizations rotate. At the SV reflection nulls, only the SH component of reflection occurs, albeit with zero amplitude at some azimuths. Thus, the reflected polarizations are 
always SH regardless of the source polarization. Outside of the SV nulls, the polarizations are pointing away from the polarizations seen next to the source. From Figure 5, we know this is because of the sign change that occurs in the SV reflectivity at this point. The $\mathrm{SH}$ zero crossings are at the very edge of the quiver plots. 


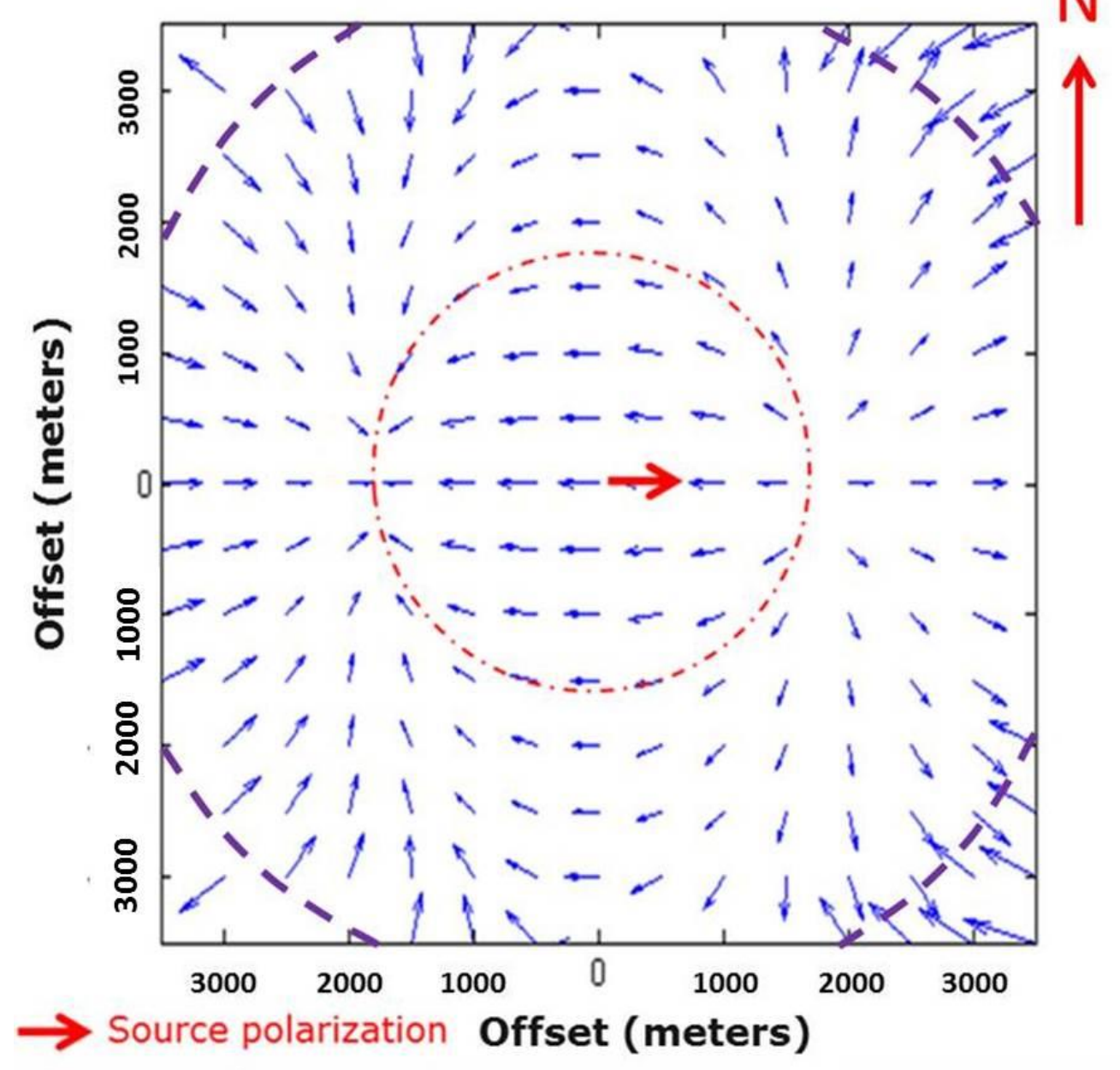

Figure 7: Simulated polarizations of shear-wave reflections in a single 3D source record (source at center) of a 3D survey of isotropic media (map view) corresponding to a reflector depth of $2000 \mathrm{~m}$. Two horizontal components (east and north) of particle motion are observed at receiver locations spatially distributed on the surface. The length of the vectors indicate the amplitude of the reflected wave, and the orientation of the vectors indicates the observed polarization (source is polarized due east). Note the wide variation in polarization over the surface. The red circle shows the offset associated with the $20^{\circ}$ zero-crossing of the SV reflection at an offset of about 1800 meters. The purple dashed lines shows the offset associated with the $40^{\circ}$ zero-crossing of the SH reflection at an offset at about 3000 meters. Note the change in polarization at the SV zero crossing position as sourcereceiver offset increases. Note the strong polarization distortion at increased offset. 


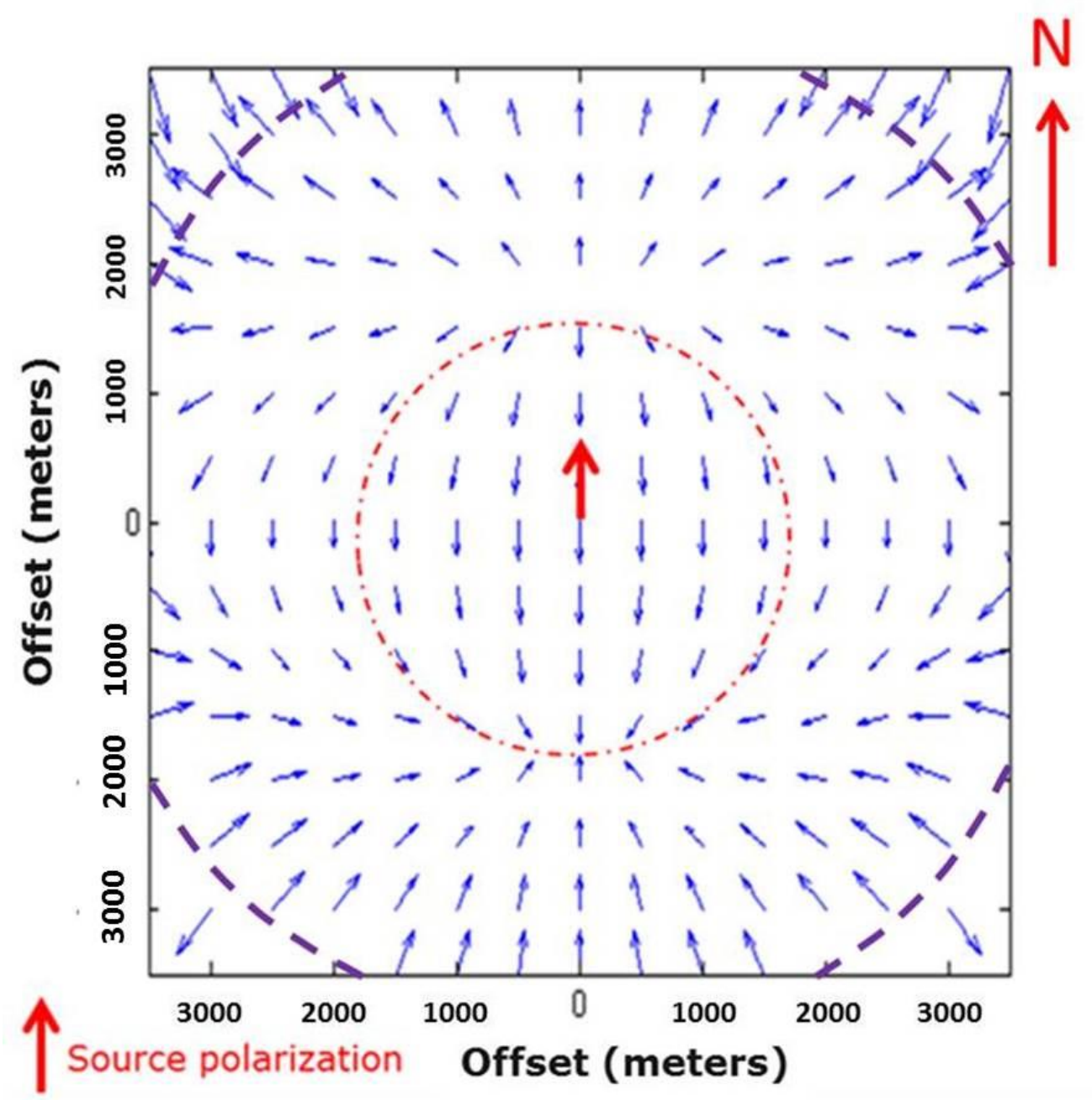

Figure 8: Computed polarizations of shear-wave reflections in a single source record 3D survey of isotropic media (map view) corresponding to a reflector depth of $2000 \mathrm{~m}$. The length of the vectors indicate the amplitude of the reflected, and the orientation of the vectors indicates the observed polarization (source is polarized due north). Note that there is a change in SV polarity. Note the wide variation in polarization over the surface. The red circle shows the offset associated with the zero-crossing of the SV reflection. The purple dashed lines shows the offset associated with the zero-crossing of the $\mathrm{SH}$ reflection. Note the change in polarization at the zero crossing position. At large offsets (4200 meters) we only SV-SV and no SH-SH because it is equivalent to the $\mathrm{SH}-\mathrm{SH}$ zero crossing value angle. 


\section{Simplification of the SV and SH reflectivity relations}

Interpretation of a correction for this polarization distortion is a major objective of this study. Further a simplification of the rather complex Zoeppritz equations is available by using published approximations of Spratt et al. (1993) and Lyons (2006) for SV-SV and SH-SH reflectivity. The full Zoeppritz equations, as examined in Aki and Richards (2002), were used simulation calculations of the full Zoeppritz curves in Figure 5 and the subsequent polarization responses in a 3D acquisition setting is shown in Figures 7 and 8 . Due to their complexity and the number of parameters required, the full Zoeppritz relations would be difficult to use to develop a workable correction for the polarization distortion effects shown in Figures 7 and 8. Further, they require detailed knowledge of seismic velocities and densities of two propagation media. Historically, several commonly applied approximations for amplitude versus angle of incidence have been developed for P-P, as well a SV-SV and SH-SH reflectivity. The most widely known for P-P reflectivity being the Shuey (1985) approximation for AVA. A similar equation has been developed for SV -wave AVA (Spratt et al., 1993). In the case of the corrections for the polarization distortion required than for the SH-SH and SV-SV reflectivity relations, these simplifications require knowledge of the values of the SH-SH and SV-SV zero crossings. Further, in addition there is remarkable little variation in the values of these zero crossing for a wide range of materials, will require a prediction of SV and $\mathrm{SH}$ reflection curves with offset.

For the simplification of the Zoeppritz relations I follow Spratt (1993) and Lyons (2006) in assuming that there is a small contrast in velocities and densities between the two layers at the interface, which yields a simplified version of equation 6 : 


$$
\grave{S} S_{S V}^{\prime}=-\frac{1}{2}\left(1-4 \beta^{2} p^{2}\right) \frac{\Delta \rho}{\rho}-\left(\frac{1}{2 \cos ^{2} j}-4 \beta^{2} p^{2}\right) \frac{\Delta \beta}{\beta}
$$

Assuming a small incident angle (generally less than $30^{\circ}$ incident angle), and a $\mathrm{Vp} / \mathrm{Vs}$ ratio of 2 (a reasonable assumption for many liquid-saturated sediments), Equation 8 simplifies to

$$
\grave{S} \dot{S}_{S V}=-R_{S}\left[7 \dot{R}_{S}+\frac{1}{2}\left(\frac{\Delta \rho}{\rho}\right)\right] \sin ^{2} j
$$

where

$$
R_{S}=\frac{1}{2}\left(\frac{\Delta \rho}{\rho}+\frac{\Delta \beta}{\beta}\right)
$$

Spratt et al. (1993) proposed an approximation that is much more manageable when we simplify it to a linear form:

$$
\mathrm{R}_{\mathrm{SV}-\mathrm{SV}}=\mathrm{A}+\mathrm{B}_{\mathrm{SV}} \operatorname{Sin}^{2} \mathrm{j}
$$

where

$$
A=-\frac{1}{2}\left(\frac{\Delta \rho}{\rho}+\frac{\Delta \beta}{\beta}\right)
$$

and

$$
B=7 R_{S}+\frac{1}{2}\left(\frac{\Delta \rho}{\rho}\right)
$$

This relation is similar in form to the Shuey's approximation (Shuey, 1985) for PP reflectivity. Figure 9 is a comparison of the full Zoeppritz curve and Spratt's SV-SV approximation from Equation 11 for the same parameters as shown in Figure 1. The approximation and the complete Zoeppritz model match closely for incidence angles of $0^{\circ}$ to $20^{\circ}$, beyond $20^{\circ}$ the approximations deviate somewhat from the Zoeppritz curves.

Spratt et al. (1993) also developed a linear $\mathrm{Sin}^{2}$ approximation to the $\mathrm{SH}-\mathrm{SH}$ Zoeppritz equations

$$
\grave{S}_{S H}=-R_{\mathrm{S}}+\frac{1}{2}\left(\frac{\Delta \beta}{\beta}\right) \sin ^{2} \mathrm{j}
$$


The SH-SH Sin ${ }^{2}$ approximation and Zoeppritz models do not agree as well with the full solution as well as the SV models, diverging significantly beyond $30^{\circ}$ (Figure 10). Further the approximation does not honor the zero crossing in the actual reflectivity curve.

$$
\mathrm{R}_{\mathrm{SH}-\mathrm{SH}}=\mathrm{A}+\mathrm{B}_{\mathrm{SH}} \sin ^{2} \mathrm{j}
$$




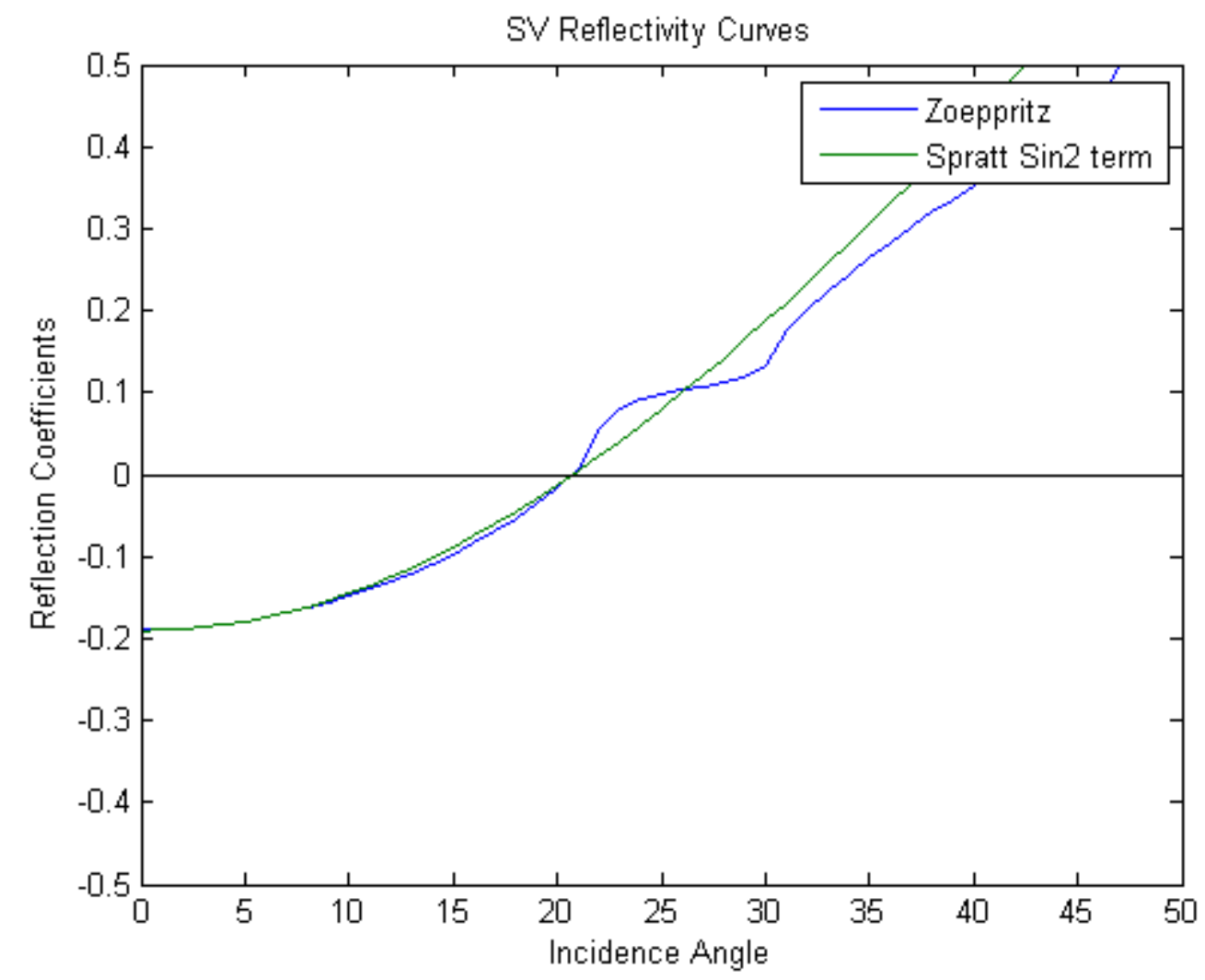

Figure 9: Comparing the results of full Zoeppritz equations to a two term $\mathrm{Sin}^{2}$ approximation, describing SV-SV reflectivity. The model parameters are listed in Table 1. There is excellent agreement between the two through the zero crossing and a very useful agreement beyond that (modified Lyons, 2006). Note the eratic deviation and from standard Zoeppritz equation because of critical angles. 


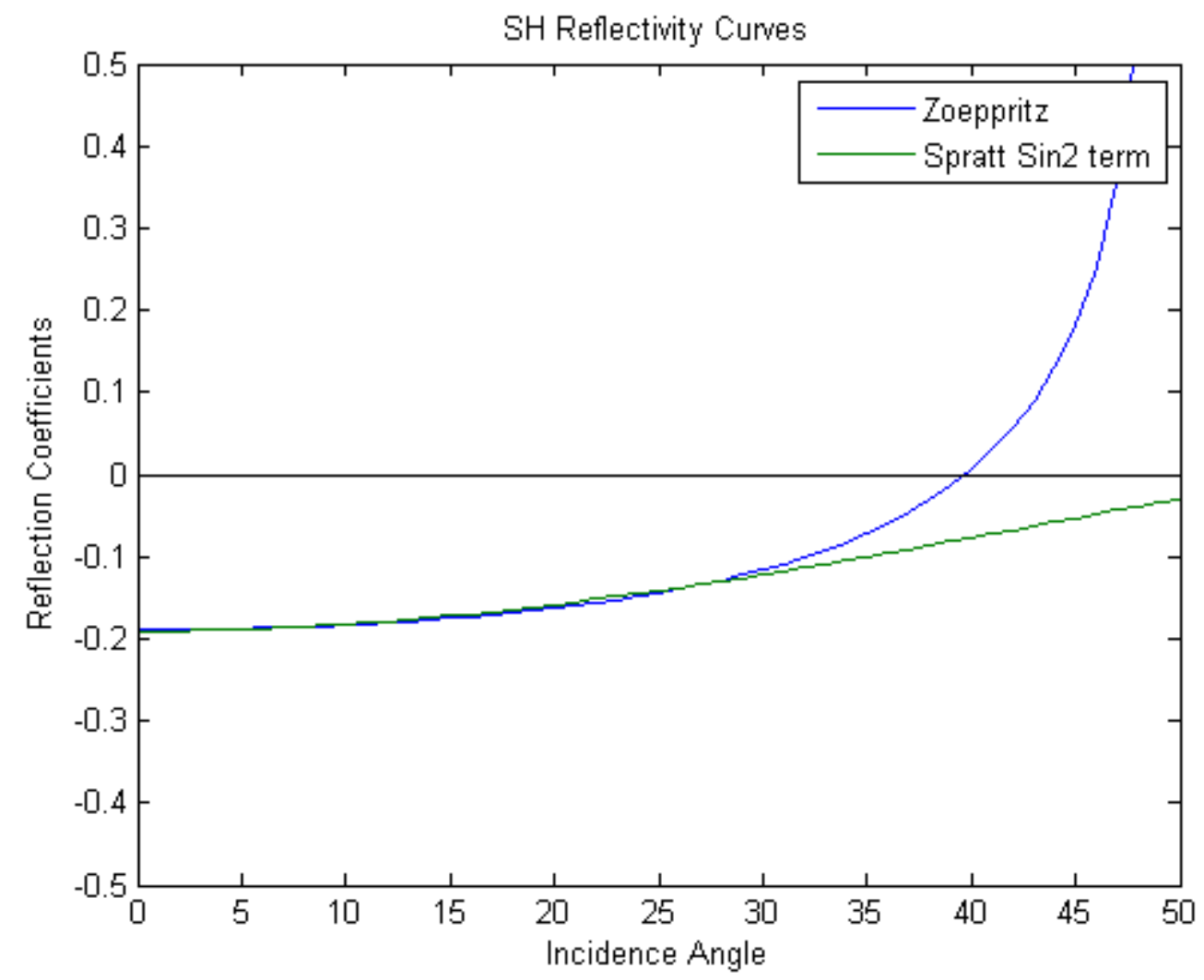

Figure 10: Comparing the full Zoeppritz equations to a two term $\sin ^{2}$ approximation, describing SH reflectivity. The model parameters are listed in Table 1 . The agreement to angles of incidence of $25^{\circ}-30^{\circ}$ is very good, but the agreement is poor beyond that and does not agree the zero crossing (elastic Brewster's angle). The lack of agreement between the two models after the zero offset reflectivity above $30^{\circ}$ is not at all useful considering we use the zero-crossing to make the correction (modified Lyons, 2006). 
To address the lack of agreement between Spratt et al. $\sin ^{2}$ approximation and the Zoeppritz equations at angles greater than $30^{\circ}$ Lyons (2006) proposed an $\mathrm{SH}$ approximation to investigate its validity in terms of $\tan ^{2}$. The fully proposed Zoeppritz equation describing SH-SH reflectivity is simple enough that it is not difficult to develop a similar approximation. Based on some of the simplifying assumptions, the modified SH-SH reflectivity approximation proposed by Lyons (2006) is:

$$
\mathrm{R}_{\mathrm{SH}-\mathrm{SH}}=\mathrm{A}+\mathrm{B}_{\mathrm{SH}} \tan ^{2} \mathrm{j}
$$

To demonstrate that the $\tan ^{2}$ approximation, rather than the $\sin ^{2}$ approximation, is appropriate for SH-SH reflectivity, a plot to compare the approximation and the Zoeppritz equation is generated (Figure 11). The approximation and the complete Zoeppritz equations for SH reflectivity is in agreement out to an incidence angle of $40^{\circ}$. In fact, this approximation honors the zero-crossing of the full Zoeppritz very well. Note, however, the divergence between the approximation and full solutions in the $\mathrm{j}=20^{\circ}-35^{\circ}$ range. 


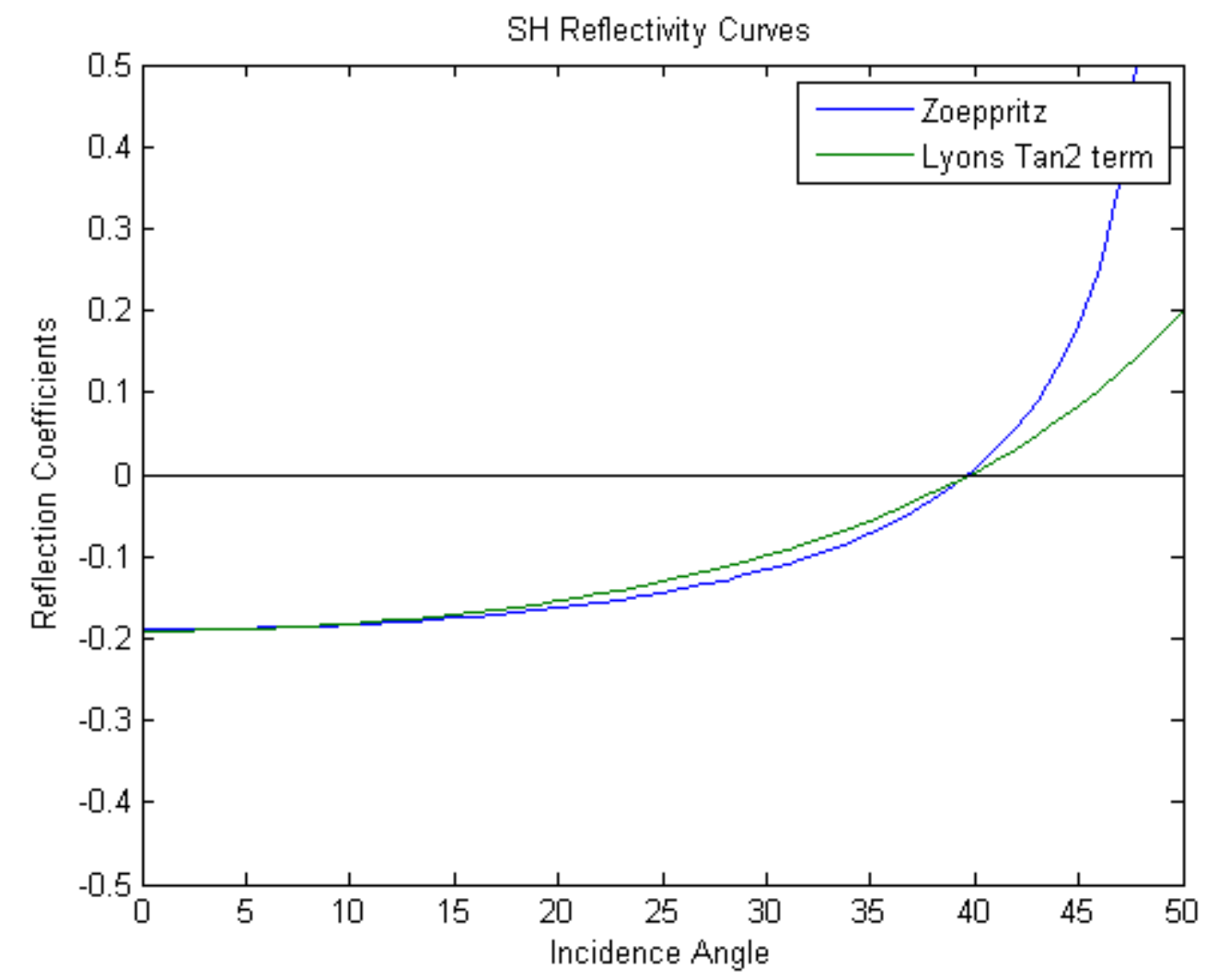

Figure 11: Comparing the full Zoeppritz equations to a two term $\operatorname{Tan}^{2}$ approximation, describing SH motion. The model parameters are listed in Table 1. Good agreement out to the zero-crossing $40^{\circ}$ suggests this is a useful approximation (modified from Lyons, 2006). Note the divergence beyond $40^{\circ}$ especially beyond the critical angle $\left(48^{\circ}\right)$.

Both the SV-SV and SH-SH reflectivity curves have clear zero crossings at angles of incidences near $20^{\circ}$ for $\mathrm{SV}$ and $40^{\circ}$ for $\mathrm{SH}$ waves. We can use these zero crossing values as a parameter to 'fit' the form of anticipated reflection curves quite well for actual reflectivity curves, B values in equation 11 and 15 may be estimated by setting reflectivity to zero at the assumed zero crossing. For the proposed amplitude correction we set $\mathrm{A}=1$, normalizing amplitudes to the zero offset values. The zero crossings define the points for developing an angle based correction. The amplitudes used to calculate the 
reflected polarization are altered to be independent of incidence angle so that the polarization seen at near-incidence is preserved, and the anomalous polarizations seen at larger angles are negated.

Since there is no polarization distortion for zero offset there is no need for the correction of the amplitude values at near offset. This is realized setting the value of the A coefficient of the correction factor to 1 . That is, no correction is applied at zero offset. The amplitudes are divided by the correction and we assume the polarization calculated by these corrected amplitudes will minimize the amplitude versus offset effect of the SV and $\mathrm{SH}$ reflectivity. Basically, these amplitude corrections remove the natural AVA variations and allow for isolation of the polarization information.

A corrected reflection coefficient for the individual SV and SH components can be used to minimize the polarization change caused by the difference in reflection of the $\mathrm{SV}$ and SH components from a direct randomly polarized S-wave source. The required $\mathrm{B}_{\mathrm{SV}}$ and $\mathrm{B}_{\mathrm{SH}}$ values can be calculated by assuming that $\mathrm{A}=1$ and $\mathrm{R}=0$ at the zero crossing values of $\mathrm{j}_{\mathrm{Z}}$. The only knowledge required for this correction is the value of the zerocrossing values. Further, jz often varies within a very limited range $18^{\circ}-24^{\circ}$ reducing the sensitivity of these required parameters to errors in estimation and variation in elastic properties and density.

Thus, for $\mathrm{B}_{\mathrm{SV}}$,

$$
0=1+B_{S V} \sin ^{2} \mathrm{j}_{\mathrm{Z}}
$$

and for $\mathrm{B}_{\mathrm{SH}}$,

$$
0=1+\mathrm{B}_{\mathrm{SH}} \tan ^{2} \mathrm{j}_{\mathrm{Z}}
$$

So

$$
B_{S V}=-1 / \sin ^{2} j_{z}
$$

and 


$$
\mathrm{B}_{\mathrm{SH}}=-1 / \tan ^{2} \mathrm{j}_{\mathrm{Z}}
$$

The correction is applied to all angles of incidence by the following equations:

$$
\begin{aligned}
& \mathrm{R}_{\mathrm{SV}-\mathrm{SVCOR}}(\mathrm{j})=\mathrm{R}_{\mathrm{SV}-\mathrm{SV}}(\mathrm{j}) *\left(1 /\left(1+\mathrm{B}_{\mathrm{SV}} \sin ^{2} \mathrm{j}\right)\right. \\
& \mathrm{R}_{\mathrm{SH}-\mathrm{SHCOR}}(\mathrm{j})=\mathrm{R}_{\mathrm{SH}-\mathrm{SH}}(\mathrm{j}) *\left(1 /\left(1+\mathrm{B}_{\mathrm{SH}} \tan ^{2} \mathrm{j}\right)\right.
\end{aligned}
$$

Where $\mathrm{R}_{\mathrm{SV}-\mathrm{SV}}(\mathrm{j})$ and $\mathrm{R}_{\mathrm{SH}-\mathrm{SH}}(\mathrm{j})$ are the observed reflection amplitude at each angle of incidence and $\mathrm{R}_{\mathrm{SV}-\mathrm{SVCOR}}(\mathrm{j})$ and $\mathrm{R}_{\mathrm{SH}-\mathrm{SHCOR}}(\mathrm{j})$ are the resultant corrected reflection amplitudes as a function of incidence angle, $\mathrm{j}$. These corrected reflectivities, $\mathrm{R}_{\mathrm{SV}-\mathrm{SVCOR}}$ and $\mathrm{R}_{\mathrm{SH}-\mathrm{SHCOR}}$ are applied to calculate the corrected amplitudes and polarizations of the reflected S-waves shown in figures 12 and 13.

These approximations and the application of the proposed correction are shown in the SV-SV and SH-SH reflectivity curves in Figures 12 and 13. It is worth noting that for the SV curve, the zero crossing has a rather narrow range of angles, for a wide variation in impedance contrast, for a wide range of interface contrasts near $20^{\circ}$. This will be discussed in the sensitivity analysis section. Overall, the SH curve tends to be rather simple with little variation at angles less than $30^{\circ}$. These two observations suggest that the use of such a simple linear form for the reflectivity curves may be readily justified, and lead to only a modest sensitivity to errors in the estimation of the zero crossings using to estimate the $\mathrm{B}_{\mathrm{SV}}$ terms in equation 15 and $\mathrm{B}_{\mathrm{SH}}$ in equation 16 .

Before proceeding with the applications of these SV-SV and SH-SH amplitude corrections to correct the polarization distortion, I will discuss the efficacy of the approximation for the SV-SV and SH-SH amplitude corrections shown in Figure 12 and 13. Since $\mathrm{R}_{\mathrm{SV}-\mathrm{SV}}$ appears in the denominator of the $\mathrm{B}_{\mathrm{SV}}$ (Equation 21) and a singularity is encountered at this angle of incidence. Thus is an unstable region near $\mathrm{j}_{\mathrm{Z}}$ for both SV and $\mathrm{SH}$, so no meaningful correction can be applied near these two angles. 
Note both corrections for Figures 12 and 13 are applied to the actual Zoeppritz curves, as well as the approximate curve for the zero-crossing of the reflectivity. Since the correction is based on the Spratt approximations the correct curve should have constant amplitude at all angles of incidence. Figure 12 shows a very close approximation between the Zoeppritz and Spratt's approximation for the SV-SV reflectivity between $0^{\circ}$ $-15^{\circ}$. Beyond $15^{\circ}$ the Spratt's approximation deviates slightly from the Zoeppritz curves between $15^{\circ}$ and the zero-crossing $\left(20^{\circ}\right)$ value. At the zero crossing both the Spratt and Zoeppritz are exactly the same (the Spratt curve assumed the same $20^{\circ}$ zero crossing) making it a consistent with the zero-crossing correction applied. Beyond the zerocrossing there is acceptable agreement between the two. The departure approaching $\mathrm{j}=22^{\circ}$ corresponds to the critical angle associated with the refracted P-wave. There are also critical angles at $30^{\circ}$ and $49^{\circ}$ corresponding to the internal $\mathrm{P}$-wave refraction in the upper layer and the shear wave refraction in the lower layer. There is also a deviation in the SVSV correction approaching the third critical angle. Also the corrections are also applied to the actual Zoeppritz curve. The black dashed line is a horizontal line showing the "perfect" correction, which observed the removal of the angle of incidence effect on the observed reflection amplitudes

Figure 13 shows the same corrections for $\mathrm{SH}-\mathrm{SH}$ reflection. Note the good agreement between the two through an incident angle of $40^{\circ}$ as the SV-SV. The correction is omitted for angles near the zero crossing. The reflection amplitude for the Lyon's approximation and the region above $40^{\circ}$ are removed because the values are too large due to the singularity at $40^{\circ}$. The black dashed line is a horizontal line showing the removal of the AVO effect of the corrected reflection amplitudes. 


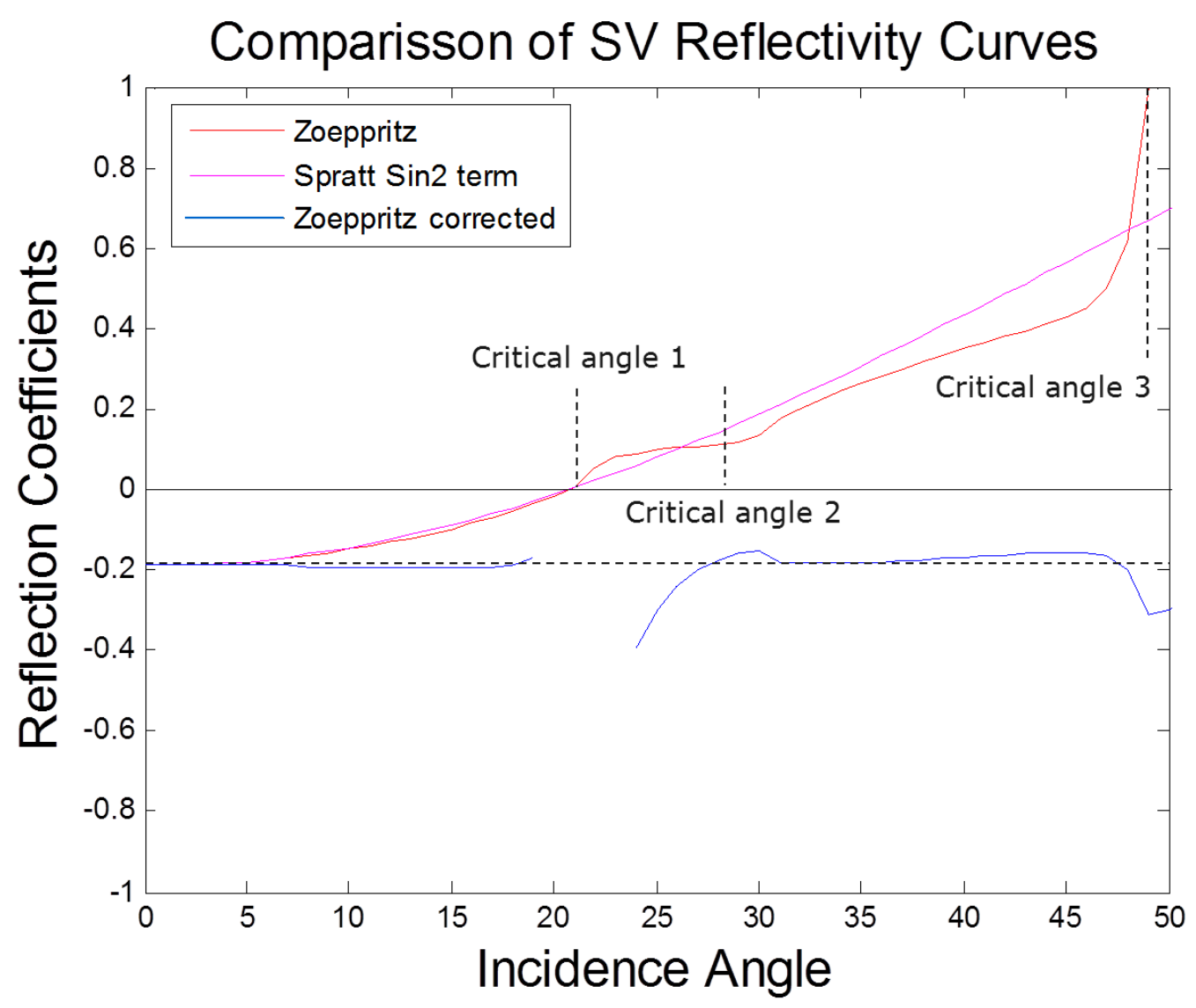

Figure 12: Comparing the full Zoeppritz equations to a two term $\mathrm{Sin}^{2}$ approximation, describing SV motion, as well as correcting the SV reflectivity for variations in amplitude with angles of incidence. The parameters for the Zoeppritz curve are listed in Table 1. There is acceptable agreement between the full Zoeppritz and Spratt approximation through an incident angle of about $30^{\circ}$. The small departure at $\mathrm{j}=22^{\circ}$ corresponds to the critical angle for the first refracted $\mathrm{P}$-wave in the second layer. There are also critical angles at $30^{\circ}$ and $49^{\circ}$ corresponding to the internal refraction in the upper layer and the shear wave refraction in the lower layer. The suggested corrections are also applied to the actual Zoeppritz curve. The unstable region near the zero crossing is omitted. The red line is the corrected reflection amplitude for the Spratt's approximation and the region between $18^{\circ}$ to $22^{\circ}$ are removed because the values are too large due to the singularity at $22^{\circ}$. The black dashed line is a horizontal line showing the removal of the AVO effect of the corrected reflection amplitudes. 


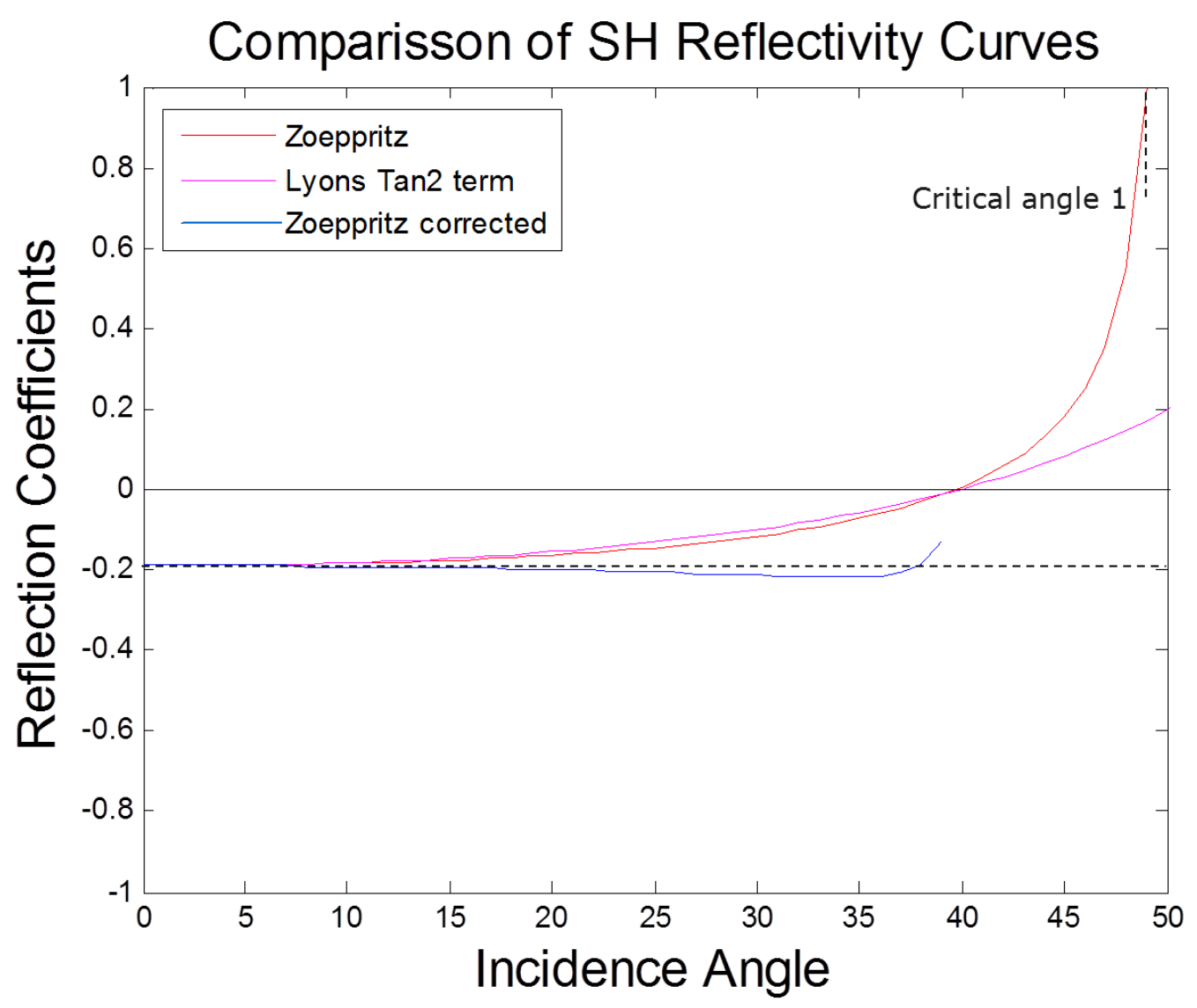

Figure 13: Comparing the full Zoeppritz equations to a two term tan approximation, describing SH reflectivity. The model parameters are listed in Table 1. There is good agreement between the actual Zoeppritz and $\operatorname{Tan}^{2}$ approximation through an incident angle of $40^{\circ}$. The suggested corrections are also applied to the actual Zoeppritz curve. The correction is omitted near the zero crossing. The red line is the corrected reflection amplitude for the Lyon's too approximation and the region above $40^{\circ}$ are removed because the values are too large due to the singularity at $40^{\circ}$. The black dashed line is a horizontal line showing the removal of the AVO effect of the corrected reflection amplitudes. 


\section{Preliminary Results on Simulated 3D data}

To demonstrate the efficacy of the correction for distorted polarization I compare the original polarization plots shown in Figure 7 and 8 with the corrected polarization plots. The corrections used a simple trigonometric relationship to calculate receiver polarization for polarization distortion calculated using equations 23 and 24 (Lyons. 2006).

$$
\begin{aligned}
& L=\tan ^{-1}\left[\frac{\cos (\theta-\psi) \mathrm{R}_{\mathrm{SV}-\mathrm{SV}}}{\sin (\theta-\psi) \mathrm{R}_{\mathrm{SH}-\mathrm{SH}}}\right]+\theta-90 \\
& L=\tan ^{-1}\left[\frac{\cos (\theta-\psi) \mathrm{R}_{\mathrm{SV}-\mathrm{SV}}}{\sin (\theta-\psi) \mathrm{R}_{\mathrm{SH}-\mathrm{SH}}}\right]+\theta+90
\end{aligned}
$$

Now we apply these corrections to the distorted polarization shown in figures 7 and 8 and obtain corrected polarizations (figure 14 and 15). Note the consistency of the polarization with respect to the original source out to rather large effects (beyond $3000 \mathrm{~m}$ or $75 \%$ reflector depth). In this region of the SV-SV zero crossing, data are "muted" to avoid instability in the correction. Also, in the region beyond the SV-SV zero crossing and the SH-SH zero crossing, phase distortions may potentially have some effect on wavelets associated with the real data. The efficiency of the polarization correction may be demonstrated in the "near" (less than the SV-SV zero crossing) range of angles and also observed at the larger angles up to the $\mathrm{SH}-\mathrm{SH}$ zero crossing. As the $\mathrm{SH}-\mathrm{SH}$ zero crossing is approached, the corrected polarization become unstable. This deviation may be more related to the effects of the third critical angle on the SV-SV reflectivity that to inadequacies of the SH-SH correction. In the corrected plots (Figures 14 and 15), all of the polarizations have the correct sign and are pointing in the direction of the source throughout. The gaps where the amplitudes are null due to the singularity at the SV-SV 
zero crossing can be observed as one concentric rings of white space this corresponds to the singularities. Also note the distortion at the ranges of the SH-SH zero crossing. Overall, the corrected polarizations are in accordance with the source polarizations. There appear to be some issues in the region of the zero-crossing in the amplitudes observed, but the goal was to remove the effect the reflection process had on the polarizations, and that has been accomplished in these simulated data with actual knowledge of the values of the SV-SV and SH-SH zero crossings. The plots for the source oriented north are identical to the plots for the source oriented east simply rotated $90^{\circ}$ counter-clockwise. Again, this distortion results from the input source not being initially aligned with the source-receiver azimuth (typical for a 3D seismic survey). At this offset (analogous to the Brewster's angle), only a reflected SH polarized wave is observed at offsets equivalent to the SV-SV zero crossing. There is a similar effect at the SH-SH zero-crossing but only $\mathrm{SV}$ is observed. 


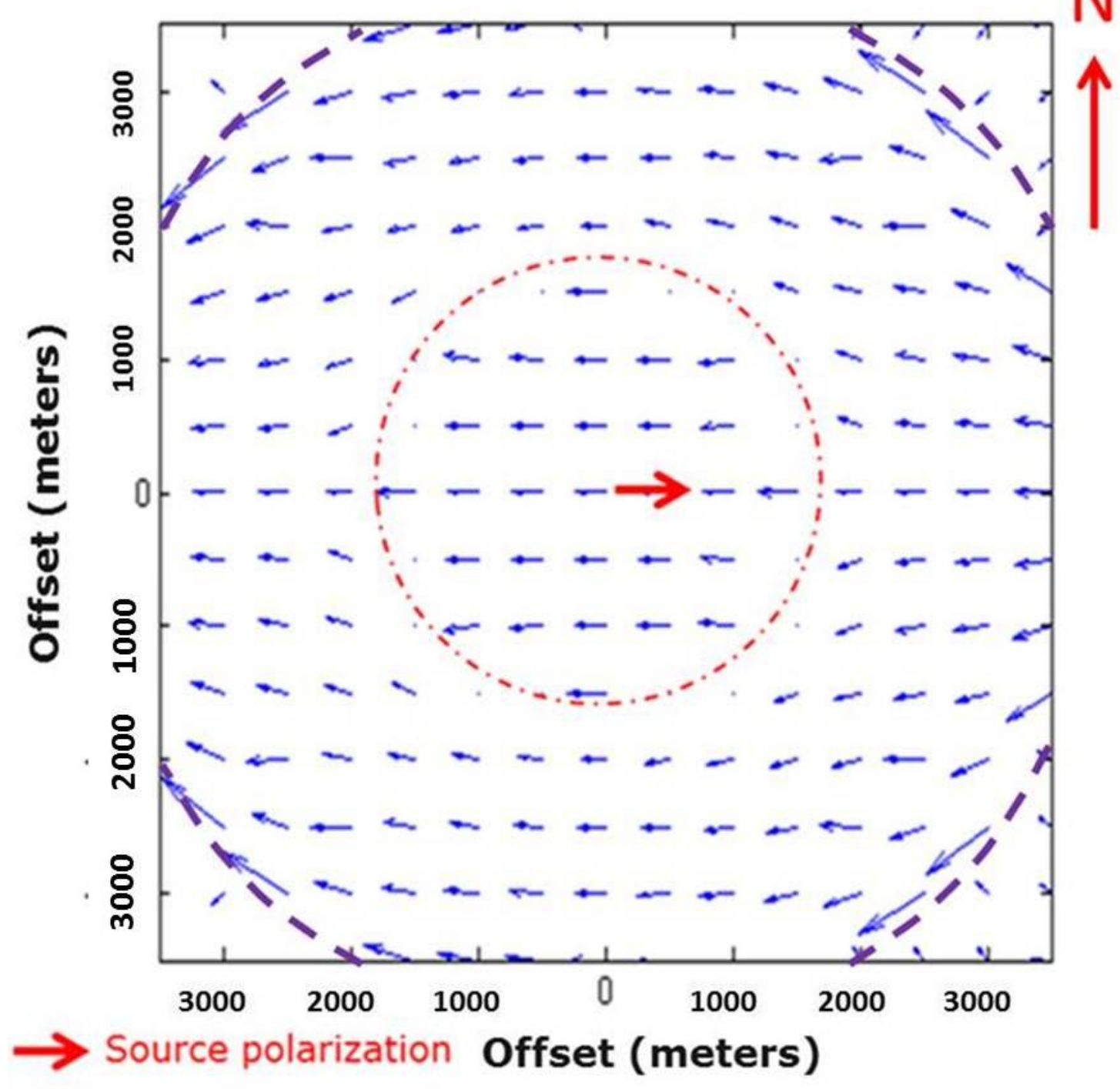

Figure 14: Polarization corrections applied to the simulated polarization distortions in a 3D record of isotropic media (map view) corresponding to a reflector depth of $2000 \mathrm{~m}$. The original polarization data is shown in Figure 7. The length of the vectors indicate the amplitude of the data, and the orientation of the vectors indicates the observed polarization (source is polarized due east). The corrections used a zero crossing of $22^{\circ} \mathrm{SV}$ and $40^{\circ} \mathrm{SH}$ from figures 12 and 13 for actual velocities used in the modeling. The purple dashed lines shows the offset associated with the zero-crossing of the SH reflection. 


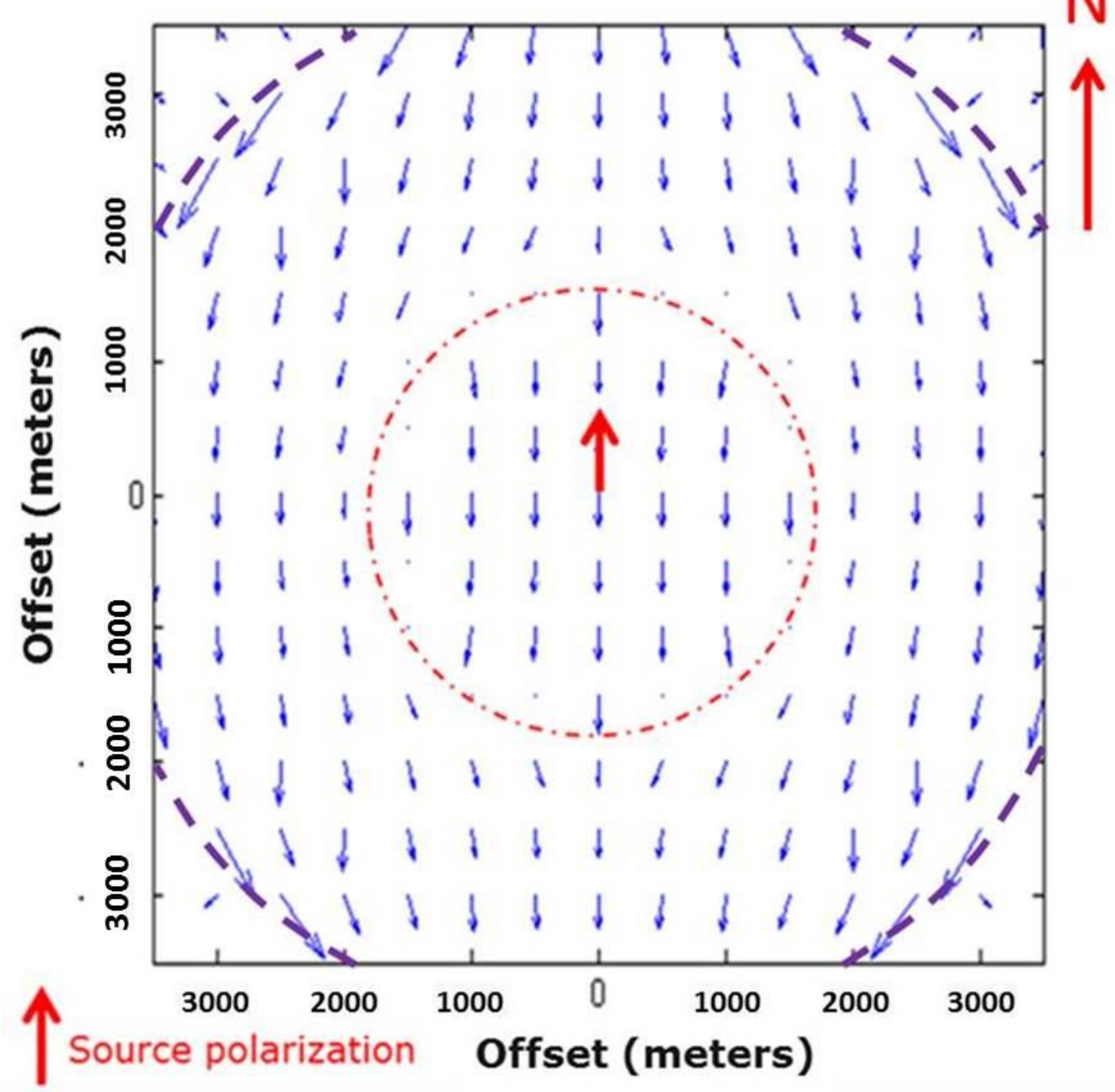

Figure 15: This plot is similar to Figure 14 with Polarization corrections applied to the simulated polarizations in a 3D survey of isotropic media (map view) corresponding to a reflector depth of $2000 \mathrm{~m}$. The original polarization data is shown in Figure 8. The length of the vectors indicate the amplitude of the corrected data, and the orientation of the vectors indicates the corrected polarization (source is polarized due north). The purple dashed lines shows the offset associated with the zero-crossing of the SH reflection. The corrections used a zero crossing of $20^{\circ} \mathrm{SV}$ and $40^{\circ} \mathrm{SH}$ from figures 12 and 13 for actual velocities used in the modeling. The only differences in Figure 14 and Figure 15 are the input source polarization correction. 


\section{Analysis of Sensitivity of variations in the Zero-Crossings}

Sensitivity analysis was performed to understand what parameters may affect the value of the elastic Brewster's angle used for the polarization correction for both SV and SH waves for different material contrasts. These angles use particularly significant in that they are the sole determining parameters for the proposed polarity correction. Figures 16 and 17, following the examples of Lyons (2006) show SV-SV and SH-SH reflectivity with contrasts in $\mathrm{V}_{\mathrm{S}}$ in a range of $+/-55 \%$ with the initial $\mathrm{V}_{\mathrm{P}} / \mathrm{V}_{\mathrm{S}}$ of 1.8 , and a constant density, of $2.2 \mathrm{~g} / \mathrm{cc}$. The $\mathrm{R}_{\mathrm{Sv}-\mathrm{Sv}}$ reflectivity is quite predictable, especially the zerocrossing being at a nearly constant value for a wide range of contrasts in $\mathrm{V}_{\mathrm{S}}$. We can hypothesize a reasonably stable correction for polarity change for a wide range of contrasts in sedimentary rocks. Even the values $j_{B}$ are quite stable for a wide range of media, the reflectivity curve itself is quite sensitive to small errors in $\mathrm{j}_{\mathrm{B}}$. Thus, a relatively broad "mute" of amplitudes is used around the singularity in the amplitude curve at $j_{B}$.

The SV and SH zero crossings are most sensitive to changes in the shear velocity contrast. The compressional velocity has a minimal effect on the value of the zero crossing. When density increases (between layers 1 and 2) the zero crossing location remains constant for SV reflectivity and a zero crossing does not exist for SH reflectivity even with changes in density (Figure 18 and 19). In Figure 16, it appears that the zero crossing for SV waves can be pegged near the $21^{\circ}$ incident angle. Note that the zero crossings considered fall in the range of $22^{\circ}$ and $28^{\circ}$.

The SH-SH zero-crossing values are not as constant as the SV-SV zero crossing values especially for Vs contrasts greater than $+/-10 \%$. Figure 17 does show that, for small contrast in Vs, the zero crossing values are near $45^{\circ}$ (for the specific models used earlier in this study, the SH-SH zero crossing was near $40^{\circ}$ ). Since the range in the zero 
crossing for $\mathrm{SH}-\mathrm{SH}$ are in the $40^{\circ}-45^{\circ}$ range, (similar to the range in SV-SV zero crossing values) and these angles ranges at near the maximum we anticipate for most surveys, I expect reasonable correction in the SH-SH reflectivity.

The zero crossing locations for $\mathrm{SH}$ waves are not as constant as the SV zero crossing locations for shear wave velocity contrasts greater than $+10 \%$. Figure 17 shows that all of the $\mathrm{SH}$ reflectivity curves occur beyond $35^{\circ}$, and for small S-wave velocity contrast are near $45^{\circ}$, I assume that $40^{\circ}-45^{\circ}$ is a reasonable range. Further since we have commonly have little data beyond $35^{\circ}$, our assumed values should yield stable results.. 


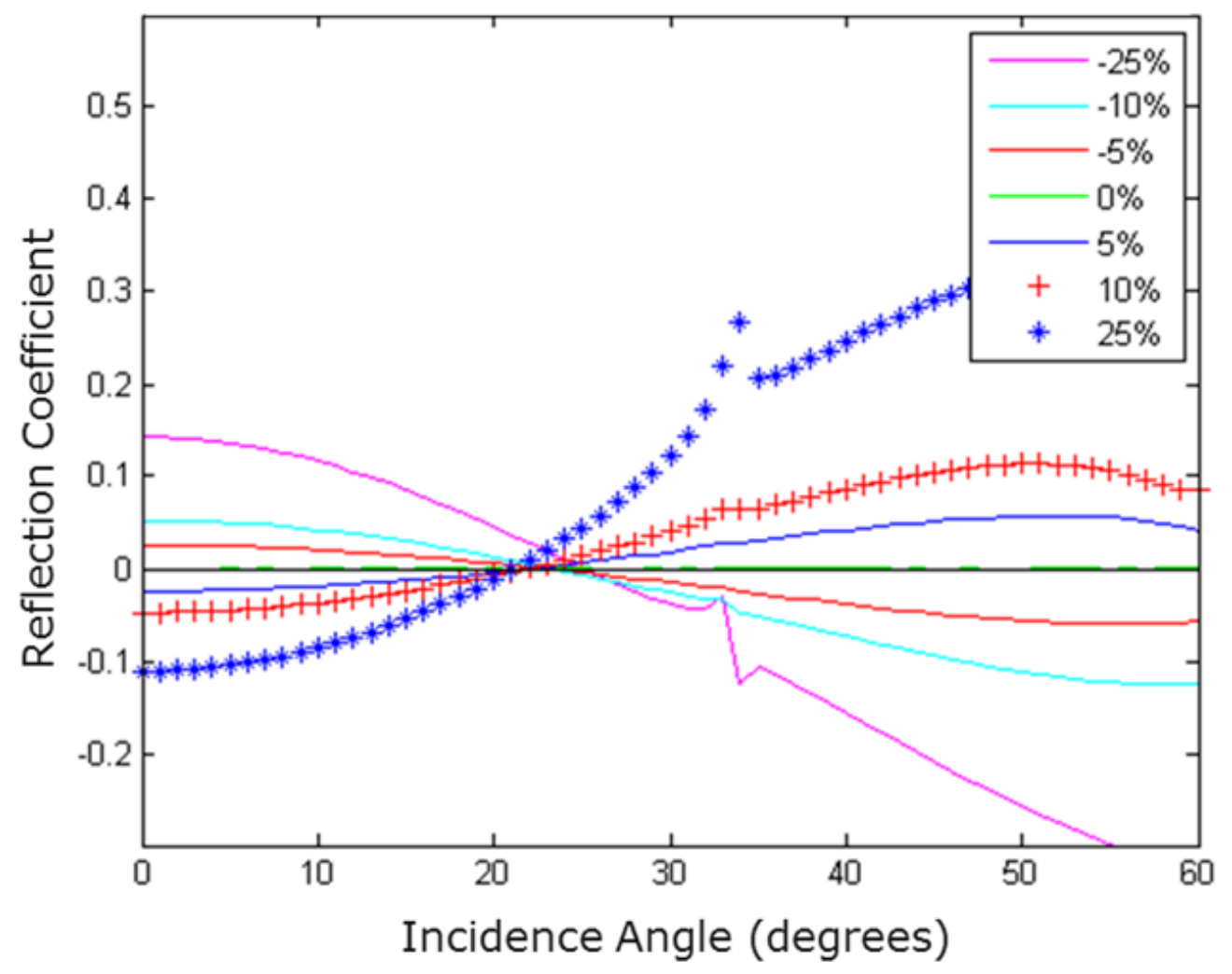

Figure 16: $\mathrm{R}_{\mathrm{SV}-\mathrm{sV}}$ reflection coefficient for incidence angles of $0^{\circ}$ to $60^{\circ}$ with changes in shear wave velocity contrast of $+/-25 \%$. Initial $\mathrm{V}_{\mathrm{P}} / \mathrm{V}_{\mathrm{S}}=1.8$, density $=$ $2.2 \mathrm{~g} / \mathrm{cc}$; density and $\mathrm{V}_{\mathrm{P}}$ is held constant and the shear wave velocity varies $+/-25 \%$ (follows Lyons (2006) Figure 3.9). Note the effect of critical angles at large contrasts in Vs. Also note the consistency of the zero-crossing near about $22^{\circ}+/-2^{\circ}$. 


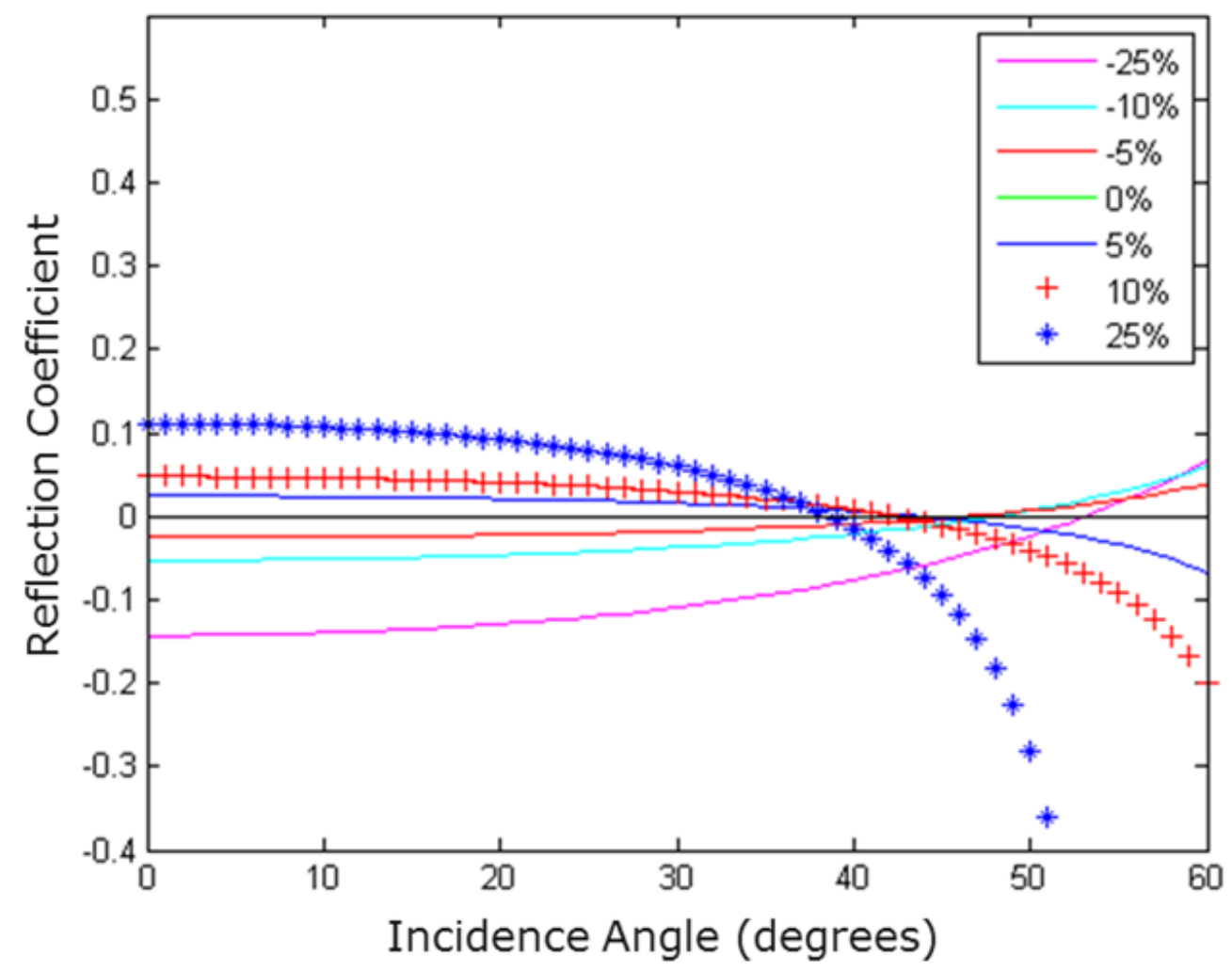

Figure 17: $\mathrm{R}_{\mathrm{SH}-\mathrm{SH}}$ reflection coefficient with changes in shear wave velocity contrast. Initial $\mathrm{V}_{\mathrm{P}} / \mathrm{V}_{\mathrm{S}}=1.8$, density $=2.2 \mathrm{~g} / \mathrm{cc}$; density and $\mathrm{V}_{\mathrm{P}}$ is held constant and the shear wave velocity varies $+/-25 \%$. The curves are quite similar with zero crossing in the range of $40^{\circ}+/-5^{\circ}$ (follows Lyons (2006) Figure 3.10). For the SH-SH reflector, the reflection coefficient is independent of $\mathrm{V}_{\mathrm{P}}$ but is dependent on density and shear wave velocity. 


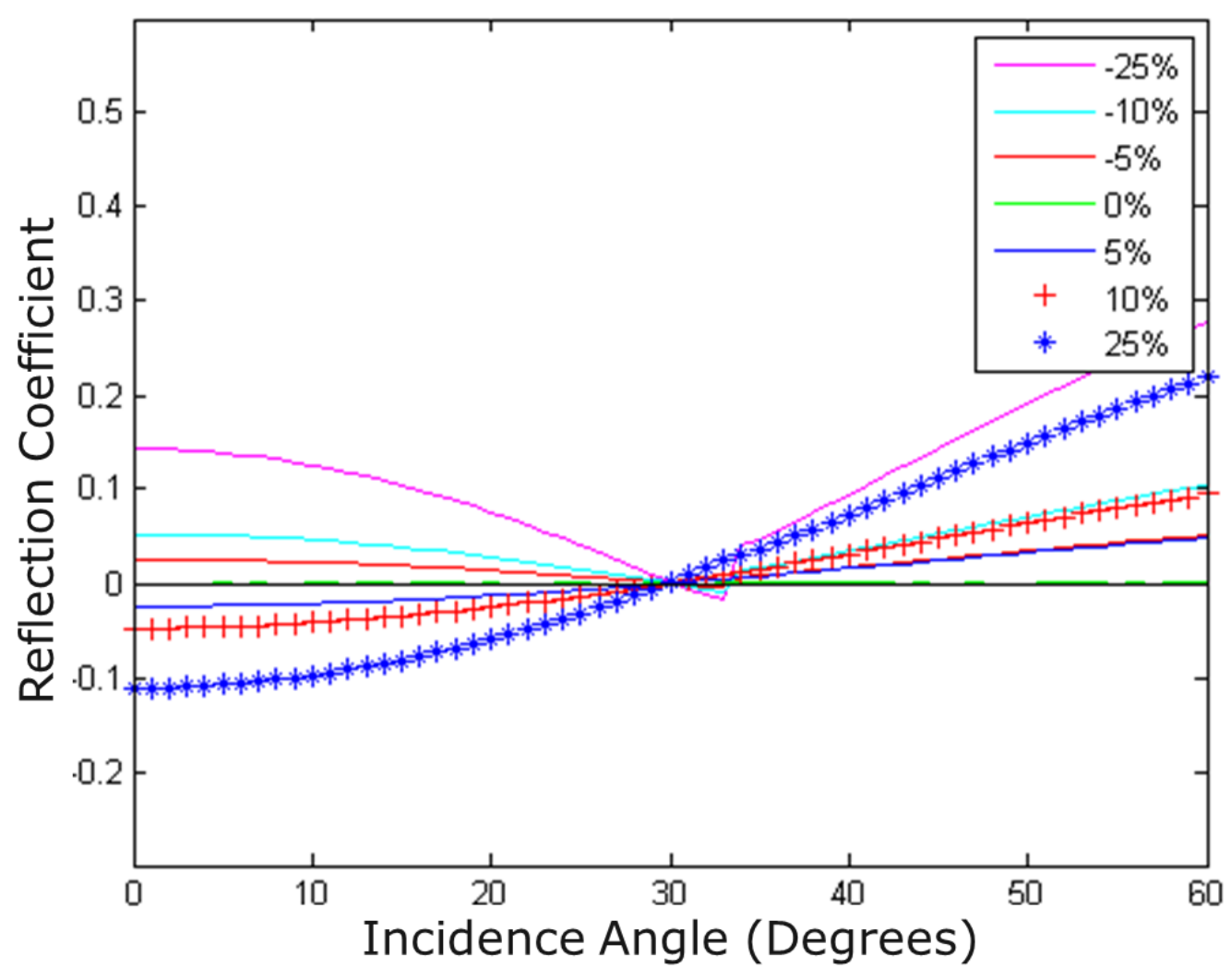

Figure 18: $R_{S V-S V}$ reflection coefficient with changes in density contrast. Initial $V_{\mathrm{P}} / \mathrm{V}_{\mathrm{S}}$ $=1.8$, density $=2.2 \mathrm{~g} / \mathrm{cc} ; \mathrm{V}_{\mathrm{P}}$ and $\mathrm{V}_{\mathrm{S}}$ is held constant and the density varies $+/-$ $25 \%$. The value of the zero-crossing is essentially independent of the density. 


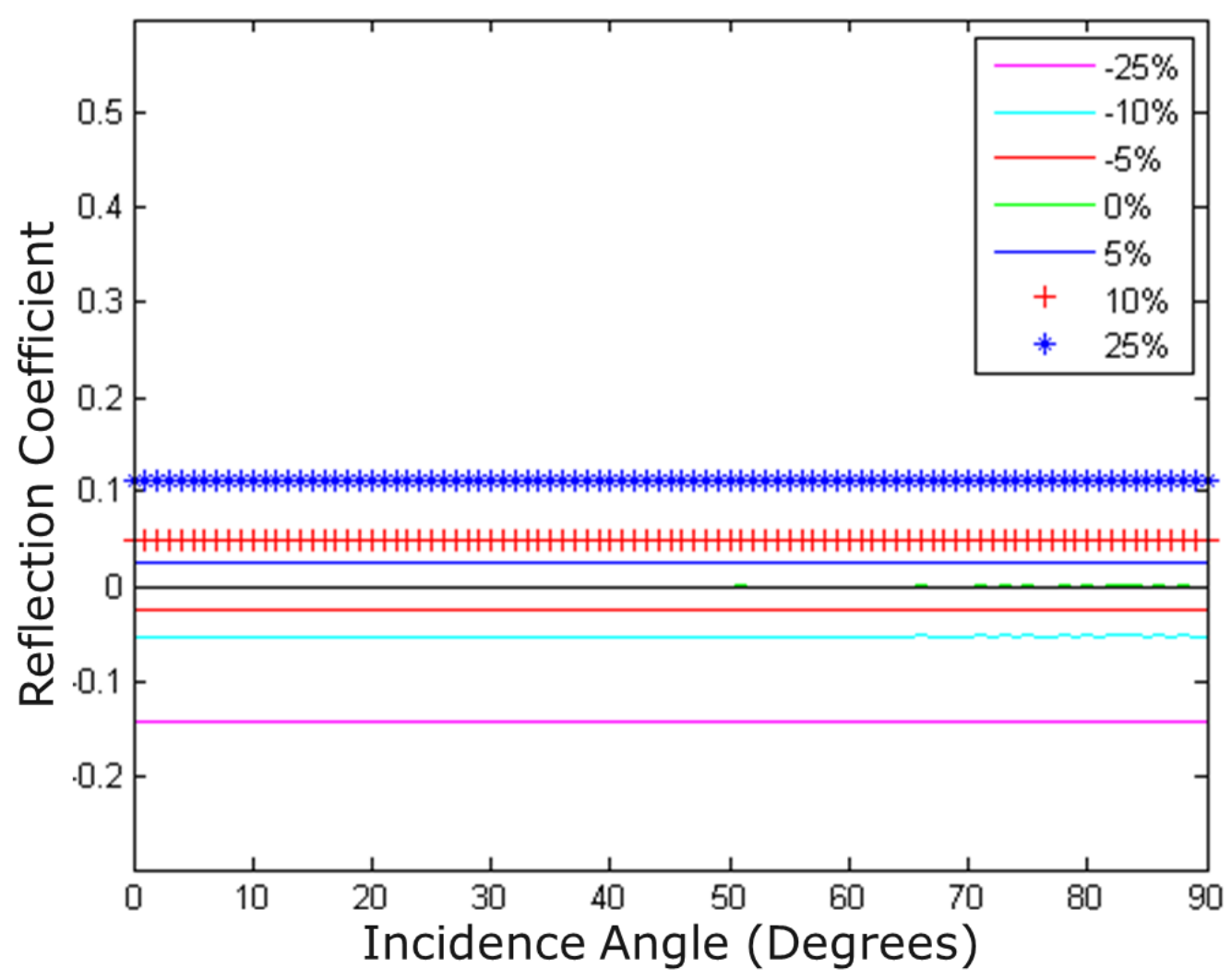

Figure 19: $\mathrm{R}_{\mathrm{SH}-\mathrm{SH}}$ reflection coefficient with changes in density contrast. Initial $\mathrm{V}_{\mathrm{P}} / \mathrm{V}_{\mathrm{S}}$ $=1.8$, density $=2.2 \mathrm{~g} / \mathrm{cc} ; \mathrm{V}_{\mathrm{S}}$ and $\mathrm{V}_{\mathrm{P}}$ is held constant and the density varies $+/$ $25 \%$.The curves are quite similar in character with no zero-crossing. 


\section{CHAPTER 2: ANISOTROPY}

The motivation for the correction to polarization distortion in the reflection process is for accommodating the analysis of shear wave polarization information for estimation of azimuthally anisotropic properties in the subsurface, particularly those characteristics that may be related to fracturing. The question comes as to how sensitive this correction really is, and what happens when we do introduce anisotropic material, especially HTI fractured media. A real data example is included and discussed in Chapter 3. Analysis of the effects and corrections for distortion in polarization for various fractured models is investigated to incorporate the polarization information for analysis of fractured media. Synthetic models are generated using locally available modeling software, which utilizes contour integration in the complex frequency-wavenumber domain to compute seismograms and thus produce interpretable reflection amplitudes (Mallick and Frazer, 1987). When the results from the synthetic models are understood then apply test the correction to a real data set from central Oklahoma.

Seismic anisotropy is the phenomenon of a medium exhibiting directionallydependent wave-propagation velocity. In the context of shear-wave propagation, anisotropy can be related to the direction of wave propagation, the direction of transverse polarization for a single propagation direction, or both. Anisotropy in sedimentary rocks may be caused by several factors, including preferred orientation of anisotropic mineral grains (e.g. clay minerals), thin (relative to seismic wavelength) bedding of isotropic layers and vertical or dipping fractures or oriented microcracks (Thomsen 1986). An excellent review of anisotropy and how it relates to lithologic and reservoir parameters is available in Thomsen (2002). Seismic S-waves, which can have essentially any polarization direction orthogonal to the propagation direction, are particularly useful in 
investigating the anisotropic "texture" of a medium using a limited range of propagation directions. Further, orthogonal components of a propagating S-wave may ultimately be polarized in the natural symmetry axes of anisotropy, specifically transversely isotropic medium. In particular, a fast shear component (S1) propagating parallel to the fracture orientation, (normal to the axis of symmetry of a transversely isotropic material) will be polarized in the direction of the higher velocity, whereas the slower shear component (S2) will be polarized orthogonal to S1 (Figure 20). Thus, for transversely isotropic media, the S1 and S2 polarization directions define anisotropic parameters which may be related to fracture or bedding orientation.

There are many simplified symmetries used to describe anisotropy that can be invoked to reduce the number of anisotropic parameters required for the analysis; these include vertically or horizontally transversely isotropic media (with a vertical axis of symmetry VTI layered media) or with a horizontal axis of symmetry, HTI (fractured media), (Tatham and McCormack, 1991). The simplest and presently commonly applied is transverse isotropy, which can be envisioned by plates, stacked either horizontally or vertically (Thomsen, 1988). Vertical fracture sets (HTI anisotropy) in the earth are a source of anisotropy in seismic velocity (Crampin, 1983; Pilant, 1979; Taylor, 1978). There may be other sources of HTI seismic anisotropy, including micro-cracks associated with stress fields. Sources of VTI anisotropy include horizontal bedding, structure and lithology (alignment of clay minerals in shale) (Tatham and McCormack, 1991). A complete description of transverse isotropy requires a total of 5 independent parameters. Isotropic elastic media can be described with only two. Intersecting sets of parallel vertical fractures and horizontal layering will result in an orthorhombic symmetry, which requires nine independent parameters. I restrict my discussion to transverse isotropy, 
mostly with a horizontal symmetry axis (HTI), which describes vertical parallel fracturing.

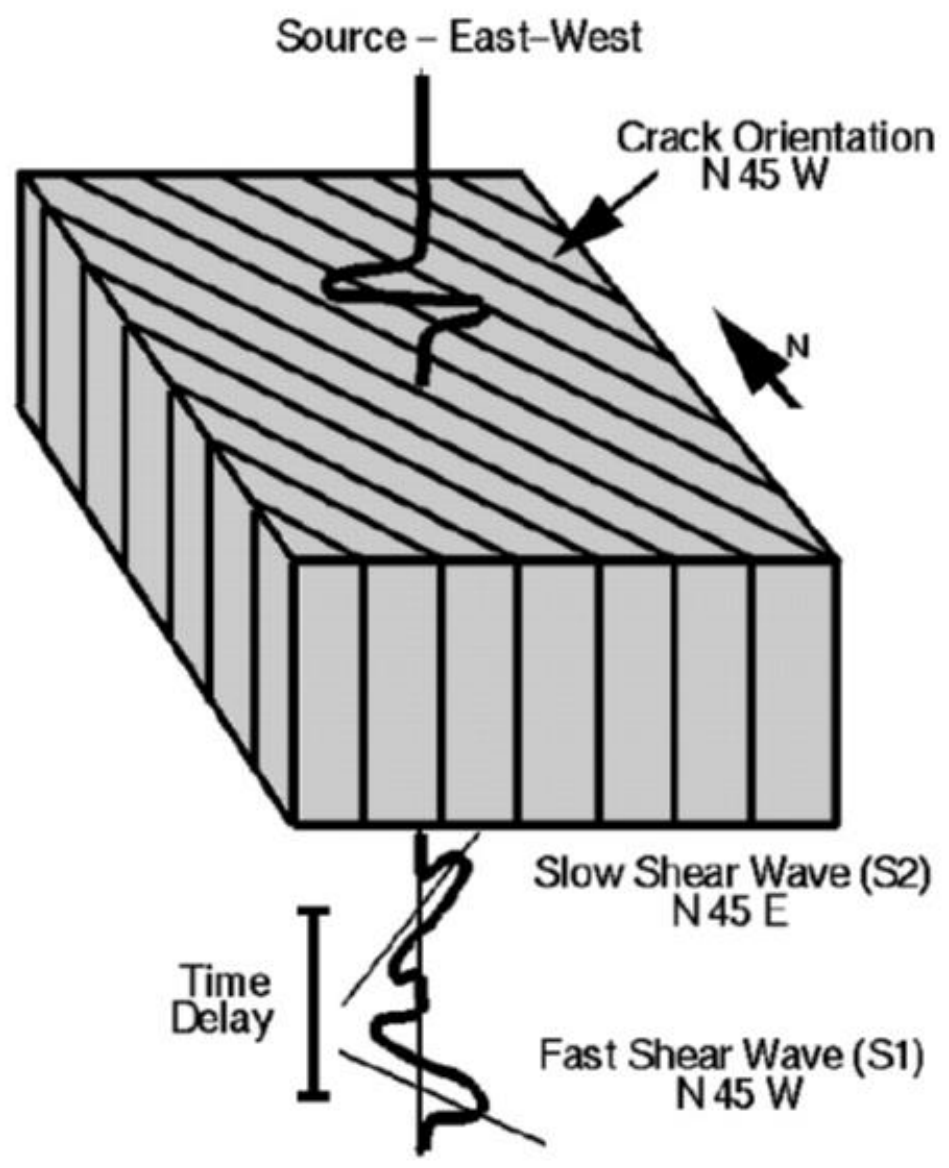

Figure 20: Model of shear wave splitting, or birefringence. An arbitrarily polarized shear wave enters the HTI (vertically fractured) medium vertically from above. Within the medium one component of the shear wave is polarized in the direction of the fractures and becomes $\mathrm{S} 1$, or the fast shear wave. The remaining orthogonal component is polarized in the direction perpendicular to the fracture orientation and becomes the slow shear wave, or S2 (modified from Bowman et al., 1987 and Martin and Davis, 1987). Thus, the incoming wave is split into two polarizations; this is identical to optical birefringence. Since the waves travel at different velocities, they separate leading to a time delay. This time delay may be related to the fracture intensity (Tatham and McCormick, 1991) 


\section{Types of Anisotropy (Transverse Isotropy)}

As introduced above, the first step away from simple, isotropic models is to consider a generally anisotropic medium. Hooke's law for a general anisotropic, linear elastic solid state that the stress $\sigma_{\mathrm{ij}}$ is linearly proportional to the strain $\varepsilon_{\mathrm{kl}}$, with a constant of proportionality as expressed the total stiffness tensor $\mathrm{c}_{\mathrm{ijk} k}$. Thus, Hooke's law can be written as where $\mathrm{i}, \mathrm{j}, \mathrm{k}$ refer to the usual Cartesian tensor coordinates 1, 2 and 3.

$$
\sigma_{\mathrm{ij}}=\mathrm{c}_{\mathrm{ijk} k} \varepsilon_{\mathrm{k} l}
$$

The elastic stiffness tensor is a fourth- rank tensor obeying the laws of tensor transformation and has a total of 81 components. Not all 81 components are independent and the symmetry of the stresses and strain imply that

$$
c_{i j k l}=c_{j i k l}=c_{i j l k}=c_{j i k l},
$$

reducing the number of independent constants to thirty-six. The existence of strain without any translation or rotation, along with some geometric limitations further reduces it the number of independent constants to 21. Further, a reduced matrix notation $\mathrm{Cij}$ can conveniently be represented as a $6 \times 6$ matrix to express the information in the fourthorder $\mathrm{c}_{\mathrm{ijkl}}$ stiffness tensor, known as the Bond transformation matrix (Mavko et. al, 2003) which is important in understanding material symmetries.

In terms of the reduced Bond notation the elastic constants these $\mathrm{C}_{\mathrm{ij}}$ 's are defined by appropriate combinations of the elements of $c_{i j k l}$ stiffness tensor. The relations between the elements $\mathrm{c}$ and the Lame's parameters $\lambda$ and $\mu$ of isotropic linear elasticity are

$$
\mathrm{C}_{11}=\lambda+2 \mu ; \mathrm{C}_{12}=\lambda ; \mathrm{C}_{44}=\mu
$$

and transversely anisotropic material can be represented with five independent constants shown below: 


$$
\mathrm{C}_{\mathrm{ij}}=\left[\begin{array}{cccccc}
C_{11} & C_{12} & C_{13} & 0 & 0 & 0 \\
C_{12} & C_{11} & C_{13} & 0 & 0 & 0 \\
C_{12} & C_{13} & C_{33} & 0 & 0 & 0 \\
0 & 0 & 0 & C_{44} & 0 & 0 \\
0 & 0 & 0 & 0 & C_{44} & 0 \\
0 & 0 & 0 & 0 & 0 & C_{66}
\end{array}\right] \text {, where } C_{66}=\frac{1}{2}\left(\mathrm{C}_{11}-\mathrm{C}_{12}\right)
$$

Now, using the Bond notation (Mavko et. al, 2003) to express vertical transverse anisotropy and horizontal transverse anisotropy in terms of elastic constants we have:

$$
\begin{aligned}
& \mathrm{C}_{\mathrm{VTI}}=\left(\begin{array}{cccccc}
C_{11} & \left(C_{11}-2 C_{66}\right) & C_{13} & & & \\
\left(C_{11}-2 C_{66}\right) & C_{11} & C_{13} & & & \\
C_{13} & C_{13} & C_{33} & & & \\
& & & C_{55} & & \\
& & & & C_{55} & \\
& & & & & C_{66}
\end{array}\right) \\
& \mathrm{C}_{\mathrm{HT}} \mathrm{I}=\left(\begin{array}{cccccc}
C_{11} & C_{13} & C_{13} & & & \\
C_{13} & C_{33} & \left(C_{33}-2 C_{44}\right) & & & \\
C_{13} & \left(C_{33}-2 C_{44}\right) & C_{33} & & & \\
& & & C_{44} & & \\
& & & & C_{55} & \\
& & & & & C_{55}
\end{array}\right)
\end{aligned}
$$

For isotropic symmetry the phase velocity of wave propagation is given by

$$
\begin{aligned}
& \beta=\sqrt{\frac{\mathrm{C}_{44}}{\rho}} \\
& \alpha=\sqrt{\frac{\mathrm{C}_{33}}{\rho}}
\end{aligned}
$$

where $\alpha$ and $\beta$ are $\mathrm{P}$-wave and S-wave velocities in the isotropic material, and $\rho$ is the density. In transversely isotropic media the wave slowness surface is always 
rotationally symmetric about the axis of symmetry. The five components of the stiffness tensor for a transversely isotropic material are obtained from five velocity measurements:

$$
\begin{gathered}
\mathrm{V}_{\mathrm{P}}\left(0^{\circ}\right), \mathrm{V}_{\mathrm{P}}\left(90^{\circ}\right), \mathrm{V}_{\mathrm{P}}\left(45^{\circ}\right), \mathrm{V}_{\mathrm{SH}}\left(0^{\circ}\right) \text { and } \mathrm{V}_{\mathrm{H}}\left(0^{\circ}\right)=\mathrm{V}_{\mathrm{SV}}\left(0^{\circ}\right), \\
\mathrm{C}_{11}=\rho \mathrm{V}_{\mathrm{P}}^{2}\left(90^{\circ}\right) \\
\mathrm{C}_{12}=\mathrm{c}_{11}-2 \rho \mathrm{V}_{\mathrm{SH}}^{2}\left(90^{\circ}\right) \\
\mathrm{C}_{33}=\rho \mathrm{V}_{\mathrm{P}}^{2}\left(0^{\circ}\right) \\
\mathrm{C}_{44}=\rho \mathrm{V}_{\mathrm{SH}} 2\left(0^{\circ}\right) \\
C_{13}=-C_{44}+\sqrt{4 \rho^{2} V_{p\left(45^{\circ}\right)}^{4}-2 \rho V_{p\left(45^{\circ}\right)}{ }^{2}\left(C_{11}+C_{33}+2 C_{44}+\left(C_{11}+C_{44}\right)\left(C_{33}+C_{44}\right)\right.}
\end{gathered}
$$

As suggested above both HTI and VTI models have the same kinematic properties as described by (Mavko et. al, 2003) the elastic constants but we can expand the analysis by using Thomsen parameters. There are greater levels of anisotropy such as orthorhombic which is characterized by three mutually orthogonal planes of mirror symmetry (Tsvankin, 2001). In the coordinate system associated with the symmetry planes, orthorhombic media have 9 independent stiffness coefficients. Such a model may be due to a combination of parallel vertical fractures with (HTI)in a horizontal layered medium (VTI). For the present discussion we focus on HTI symmetry because we are dealing with direct shear wave sources and vertical fractures in order to correct for polarization corrections. 

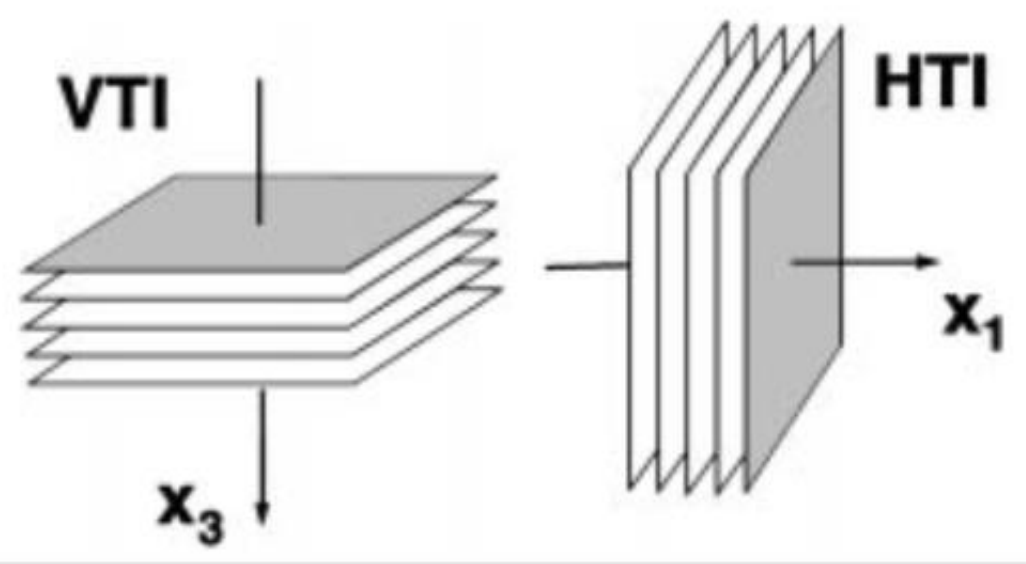

Figure 21: Sketch of VTI and HTI models. Reflections of P-waves confined to the two vertical symmetry planes, here called the symmetry-axis plane and the isotropy plane. Shear waves polarized parallel and normal to the isotropy plane have different vertical velocities (Rüger, 1997).

\section{THOMSEN'S PARAMETERS}

Thomsen (1986) introduced the concept of "weak" anisotropy, as a means of simplifying parameters describing transverse isotropy. This simplification is particularly useful in reflection seismology for exploration applications, where incident angles are typically on the order of $<35^{\circ}$. Thomsen's motivation(s) for developing this theory included: 1) the inadequacy of measurements of only horizontal and vertical velocities for use in problems utilizing near vertical $\mathrm{P}$-wave velocity paths to describe anisotropy, 2) the lack of experimentally derived results for $\delta$, one of the five parameters required for fully describing transverse isotropic media ( $\delta$ is very difficult to estimate for most experimental observations limited by observed propagation angles) 3) the inadequacy of the commonly applied elliptical approximation for P- and SV-wave propagation, and 4) 
the error incurred from using Poisson's ratio from vertical velocities to estimate the horizontal stress field.

Thomsen's weak anisotropy assumptions pertains solely to transverse anisotropy incurred from a set of preferentially oriented vertical cracks, or a series of isotropic horizontal beds (Schoenberg and Muir 1989). This development was carried out with azimuthal anisotropy in mind, but most results are to be expandable more complex symmetries (Thomsen, 2002). In terms of the P-wave and S-wave velocities, $\alpha$ and $\beta$, propagating along the symmetry axis plus three additional constants derived on elements of the $6 \times 6$ Voigt expression of elastic stiffness tensor described in this section. These parameters were initially:

$$
\begin{gathered}
\varepsilon=\frac{C_{11}-C_{33}}{2 C_{33}}=\frac{V_{p}\left(90^{\circ}\right)-V_{p}\left(0^{\circ}\right)}{V_{p}\left(0^{\circ}\right)} \\
\gamma=\frac{C_{66}-C_{44}}{2 C_{44}}=\frac{V_{S H}\left(90^{\circ}\right)-V_{S V}\left(90^{\circ}\right)}{V_{S V}\left(90^{\circ}\right)}=\frac{V_{S H}\left(90^{\circ}\right)-V_{S H}\left(90^{\circ}\right)}{V_{S H}\left(90^{\circ}\right)} \\
\delta=\frac{1}{2 C_{33}^{2}}\left[2\left(C_{13}+C_{44}\right)^{2}-\left(C_{33}-C_{44}\right)\left(C_{11}+C_{33}-2 C_{44}\right)\right]
\end{gathered}
$$

The phase angle between the normal to the wavefront and the symmetry axis (z-axis) is denoted by $\theta$. Hence $\theta=90^{\circ}$ and $\theta=0^{\circ}$ are the velocities in the vertical and horizontal directions respectively (Tsvankin, 2001). The anisotropy constant $\varepsilon$ (same symbol was used for strain) can be seen to describe the fractional difference of the P-wave velocities in the vertical and horizontal directions, therefore usually called the P-wave anisotropic factor. Anisotropic parameter gamma, $\gamma$ can be described as the fractional difference of 
SH velocities between the vertical and horizontal directions, or the S1 and S2 polarizations for the same ray paths.

It has also been demonstrated that it is possible to determine $\delta$ from a single set of measurement (where $\theta=0^{\circ}, 45^{\circ}$ and $90^{\circ}$ ). The experimental difficulty is getting $\mathrm{V}$ for $\theta=$ $45^{\circ}$, Thomsen parameters may also more accurate than estimates made utilizing Poisson's ratio derived from vertical velocities (Thomsen, 1988). In well logging when dealing with thin, fractured reservoirs, it is imperative to have a appropriate vertical resolution anisotropic acquisition tool because of the effects of thin beds. Shear wave travel times have been demonstrated to be that tool. Utilizing travel time measurements of fast and slow S-waves (S1 and S2) it is possible to detect anisotropy of a medium, when it is greater than $1 \%$, and relate it to fracture intensity for a sample of secetion overs 10's of meters (Thomsen, 1988).

Although useful, Thomsen's results may be specifically limited to transversely isotropic medium depending on the acquisition geometry of the experiment. Some results are also restricted to the subclass of HTI where anisotropy is limited to a single set of vertically aligned fractures (figure 22). Hence we use Thomsen parameters in describing a model of an isotropic medium over an anisotropic medium in order to extract fracture direction and fracture density in the lower medium. Thomsen parameters were also varied from $1 \%$ to $15 \%$ in order to determine what level of anisotropy dominates the reflection process. Since in an HTI medium the polarity of vertically propagating shear waves take on the orientation of the HTI symmetry, I limit this model to anisotropic upper layer where the shear-waves actually propagate. 


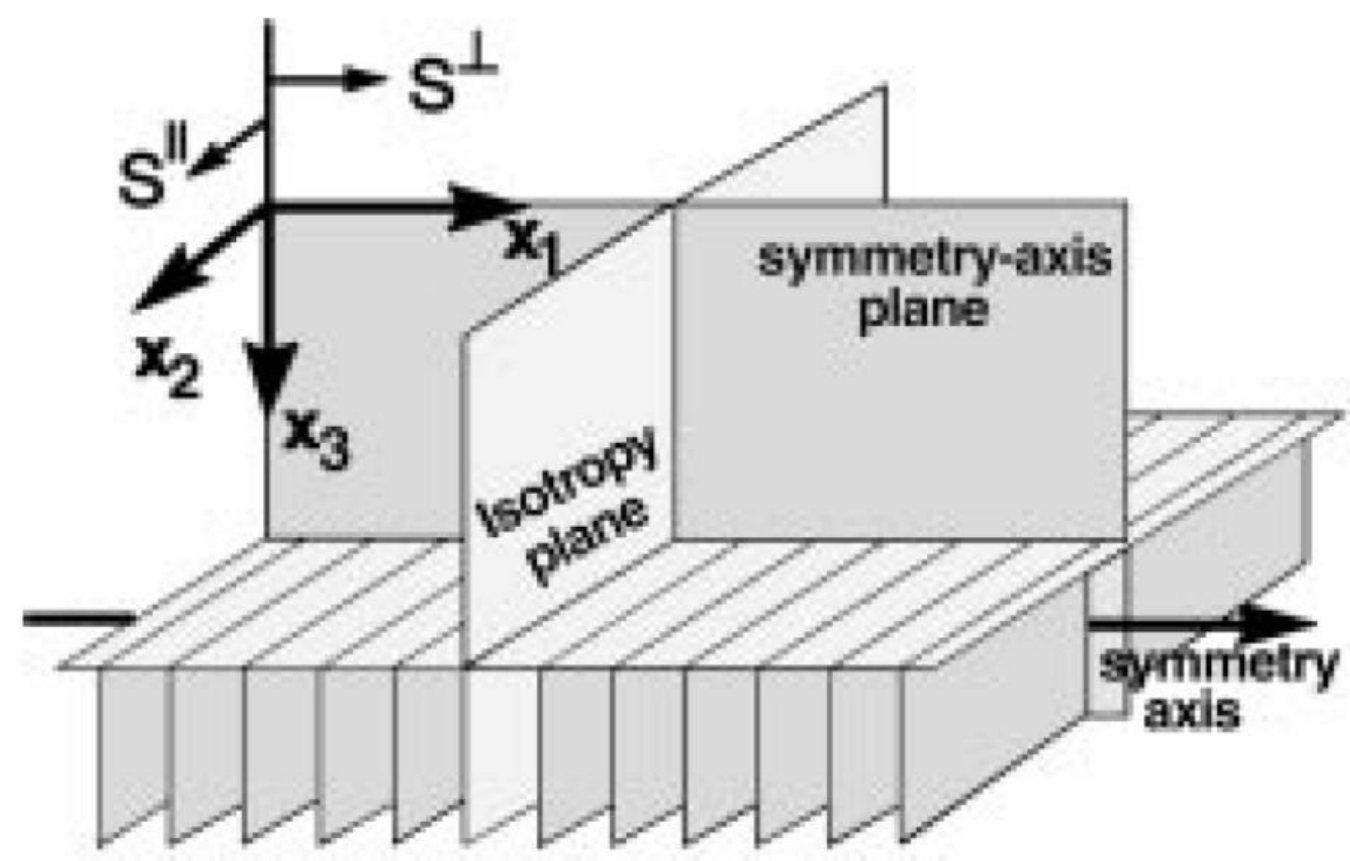

Figure 22: Sketch of the geometry associated with an isotropic layer over an HTI model. Reflections of S-waves polarizations confined to the two vertical symmetry planes, here called the symmetry- axis plane and the isotropy plane, are discussed in the text. Vertically propagating shear waves polarized parallel and normal to the isotropy plane have different vertical velocities in the lower (HTI) medium, but equal velocities in the upper isotropic medium (Rüger, 1996). 


\section{Shear waves and Azimuthal AVO in Transversely Isotropic Media}

Analysis of direct shear wave reflection data is a promising tool in understanding fracture characterization not only because of shear-wave splitting (birefringence) in the presence of transverse isotropy, it also creates some challenging complications in processing the shear-wave data (Lynn et al., 1995; Kendall and Kendall, 1995). Because we are assuming HTI anisotropy for fracture analysis we may approximate the HTI anisotropy by using the Thomsen (1986) coefficient $\gamma$, may be estimated by time difference in the shear wave splitting into the fast (S1) and slow (S2) shear waves. Gamma $(\gamma)$ can also be estimated by measuring P-velocity in various directions but may subject to issues of multiple propagation paths due to inhomogeneity and the limitation that magnitude of the P-wave anisotropy is not always equivalent to the magnitude of the S-wave anisotropy. Because we are dealing with shear waves, azimuths for analysis of reflection effects will be performed in the 2-D acquisition symmetry plane and in the 2-D isotropy plane (Figure 22), following the work of Rüger (1996) and associates with the HTI medium.

The simulated acquisition geometry described in Figure 23 shows the shear-wave polarizations for a multicomponent survey with two different 2D profiles defining planes in which the S-waves particle motions are confined. Knowing which planes the S waves are polarized in is important for describing the polarization distortion analysis because it determines which planes describe SV and SH basis for analysis. Keep in mind that SV

and SH are defined for each plane $\mathrm{S} \|(\mathrm{S} 1)$ and $S^{-}-{ }_{-}$(S2), and are associated solely with the propagating wave. The zero-crossing angles in the SV-SV and SH-SH reflectivity are considered in each these survey planes (Rüger, 1996). The diagram shows that if the horizontal shear source is oriented in the strike direction of the fractures it generates a $\mathrm{S} \|$ 
wave, or - a wave polarized within the fracture plane. As noted the $\mathrm{S} \|$ wave polarization is confined to the fracture plane (here denoted as the isotropy plane), whereas the $S^{-} \mathrm{I}_{-}$ wave is polarized in the plane formed by the slowness vector and the symmetry axis.

For a horizontal shear source oriented in the strike direction the approximate reflection coefficient, this is identical to the isotropic SV-wave reflection coefficient for shear waves.

$$
\begin{gathered}
R_{S \|}^{\text {strike }}=R_{S V}^{i s o}=S_{1} \\
R_{S V}^{i s o}=-\frac{1}{2} \frac{\Delta Z^{S}}{\bar{Z}^{S}}+\left(\frac{7}{2} \frac{\Delta V_{S}}{\bar{V}_{S}}+2 \frac{\Delta \rho}{\bar{\rho}}\right) \sin ^{2} j-\frac{1}{2} \frac{\Delta V_{S}}{\overline{V_{S}}} \sin ^{2} j \tan ^{2} j
\end{gathered}
$$

thus defined the velocity of the shear wave in the fracture strike orientation, is defined as $V_{S}=\sqrt{C_{44} / \rho}$.

For a $2 \mathrm{D}$ profile aligned with the fracture strike direction defined as $R_{S H}^{I S O}, S^{-\mathrm{I}_{-}}, \mathrm{S}_{2}$ is polarized perpendicular to the vertical plane. Hence $\mathrm{SH}$ wave becomes isotropic

$$
\begin{aligned}
& R_{S_{-} l_{-}}^{\text {strike }}=R_{S H}^{i s o}=S_{2} \\
& R_{S H}^{i s o}=-\frac{1}{2} \frac{\Delta Z^{S}}{\bar{Z}^{S}}+\frac{1}{2} \frac{\Delta V_{S}}{\bar{V}_{S}} \tan ^{2} j
\end{aligned}
$$

where

$$
Z^{S}=\rho V_{S}
$$

with shear wave normal velocity $R_{S H}^{i s o}$ being defined as $V_{S}=\sqrt{C_{55} / \rho}$. ZERO-CROSSING USING RÜGER’S EQUATION

Now we expand the model to an isotropic medium over an anisotropic (HTI) medium for 2D profiles in strike and symmetry directions. Direct shear data is simulated in a three dimensional model of an isotropic layer over an anisotropic medium using a 
Fourier frequency-wave number numerical modeling method (Mallick and Frazer, 1987). Both orthogonal components of $\mathrm{SV}$ and $\mathrm{SH}$ reflections go through a zero crossing at about $22^{\circ}$ and $40^{\circ}$, respectively, for the isotropic over the isotropic layer. When we expand to an isotropic model over anisotropic model, the zero crossing locations vary by a few degrees due to fast and slow shear waves being developed in the lower half space.

Figure 23 shows different acquisition profile directions and with these directions we gain different shear wave reflection coefficients for both SV-SV and SH-SH reflections. Let's first discuss a 2D acquisition profile with an acquisition profile that is along the fracture strike direction. From Rüger, 2001 the SV-SV and SH-SH reflection coefficient are described above in equation 32 and 34. If we apply the same analysis to a shear wave propagating or polarized normal to fracture direction, the Rüger 2001 has a different result:

$$
\begin{gathered}
R_{S \|}^{\text {sym }}=R_{S H}^{i s o}+\frac{1}{2}\left(\Delta \gamma^{(V)}\right) \tan ^{2} j=S_{1} \\
R_{S \perp}^{s y m}=R_{S V}^{i s o}+\frac{1}{2}\left(\frac{\bar{V}_{p}}{V_{S}^{\perp}}\right)^{2}\left(\Delta \varepsilon^{(V)}-\Delta \delta^{(V)}\right) \sin ^{2} j=S_{2}
\end{gathered}
$$

The primary wave and the shear wave propagate in the $\mathrm{x} 1, \mathrm{x} 3$ planes (Figure 21) in the symmetry-axis plane of the HTI media can be described using the elastic stiffness components $\mathrm{c}_{\mathrm{ij}}$ (described in chapter 2) using the Thomsen parameters $\varepsilon^{(\mathrm{V})}$ and $\delta^{(\mathrm{V})}$ of the equivalent VTI model are different from the generic coefficients $\varepsilon$ and $\delta$ defined with respect to the horizontal symmetry axis (HTI).

$$
\varepsilon^{(V)} \equiv \frac{C_{11}-C_{33}}{2 C_{33}}
$$




$$
\delta^{(V)} \equiv \frac{\left(C_{13}+C_{55}\right)^{2}\left(C_{33-} C_{55}\right)^{2}}{2 C_{33}\left(C_{33}-C_{55}\right)}
$$

Table 2 describes four models where I applied Rüger's equations to calculate the reflection coefficients for an isotropic medium over an HTI medium in the symmetry and strike planes (Rüger, 2001) with varying Thomsen's anisotropic parameters. I estimated the zero crossing locations, which are shown in Figure 24, for the various models and these values, of zero-crossings were used to correct for polarization distortion. This is consistent with the relative reliability and narrow range of the zero crossing values for a source parallel and for a source perpendicular to HTI axis symmetry in the varying models. This reasonable consistency in the zero-crossing values may lead to the possible to evolution of a standard or at least stable correction and may potentially be applied for sources parallel to strike direction and sources normal to strike direction for varying levels of anisotropy.

The $\mathrm{SH}$ and $\mathrm{SV}$ zero crossings were used to minimize the amplitude change caused by the distortion upon reflection of the S-wave from a direct S-wave source. The required $B_{S V}$ and $B_{S H}$ values can be calculated by assuming that $A=1$ and $R=0$ for the zero crossing values of the respective medium of interest (Table 2) for SV and $\mathrm{SH}$ respectively. Thus following chapter 1:

$$
0=1+\mathrm{B}_{\mathrm{SV}} \sin ^{2} \mathrm{j} \mathrm{Z}
$$

and

$$
0=1+\mathrm{B}_{\mathrm{SH}} \tan ^{2} \mathrm{j}_{\mathrm{Z}}
$$

So

$$
B_{S V}=-1 / \sin ^{2} j_{z}
$$

and 


$$
\mathrm{B}_{\mathrm{SH}}=-1 / \tan ^{2} \mathrm{j}_{\mathrm{Z}}
$$

The correction is applied by implementing the following equations for a source acquisition parallel to fracture strike:

$$
\begin{aligned}
& \mathrm{R}_{\mathrm{SV}-\mathrm{SVCOR}}=R_{S \| l}^{\text {strike }} *\left(1 /\left(1+\mathrm{B}_{\mathrm{SV}} \sin ^{2} \mathrm{j}\right)(38)\right. \\
& \mathrm{R}_{\mathrm{SH}-\mathrm{SHCOR}}=R_{\left.S_{-}\right|_{-}}^{\text {strike }} *\left(1 /\left(1+\mathrm{B}_{\mathrm{SH}} \tan ^{2} \mathrm{j}\right)\right.
\end{aligned}
$$

We use the corrected reflection coefficients to correct for the polarization distortion that occurs in the isotropic over HTI anisotropic medium. 


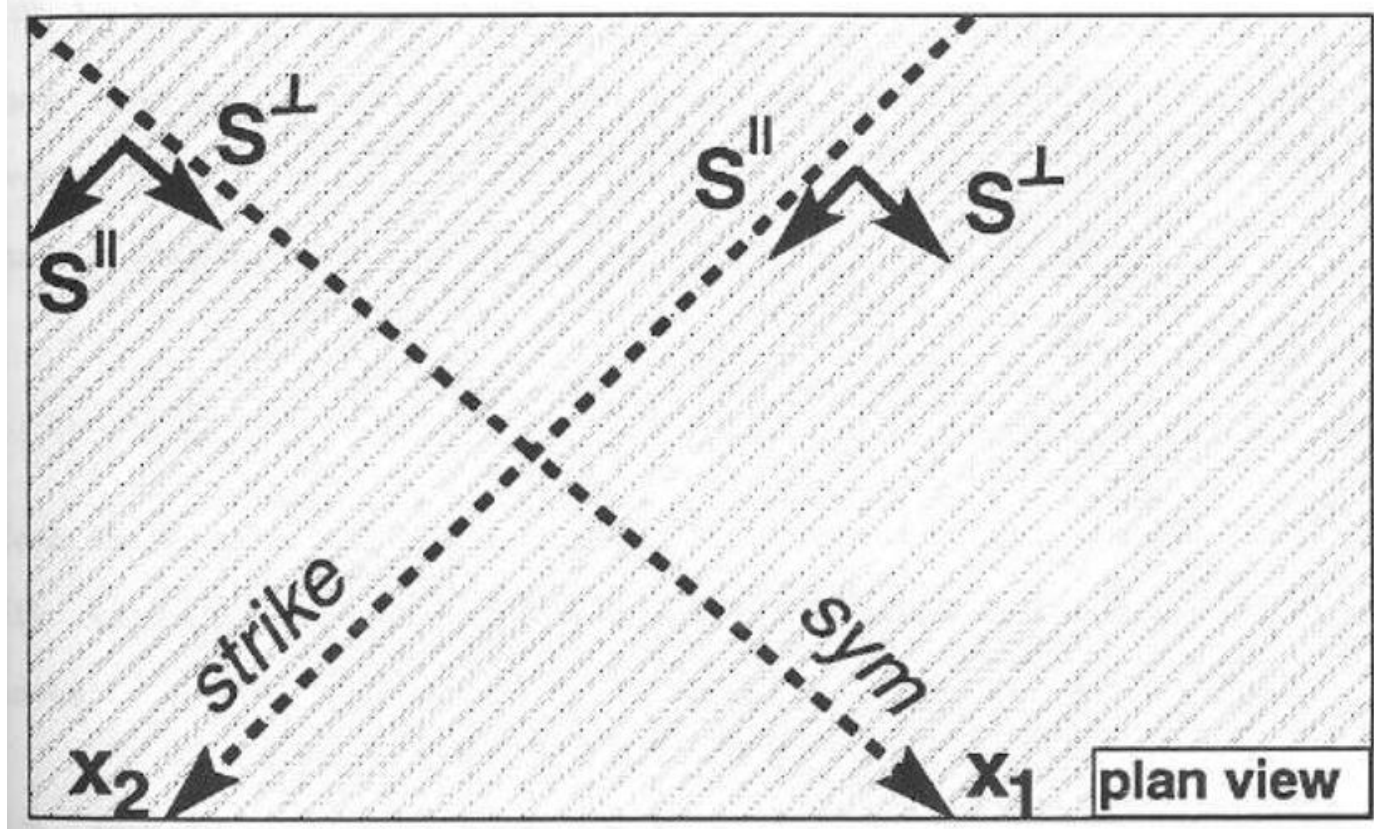

Figuüe 23: Plan view of HTI medium, with the source and receiver acquisition profiles fracture strike and HTI symmetry direction and the polarization direction for the fast and slow shear waves. Amplitude analysis as a function of angle of incidence in the isotropic layer will be performed in the strike and symmetry profile directions which represent the isotropy and the symmetry-axis planes, respectively. In this nomenclature $\mathrm{S} \|=\mathrm{S} 1$, the polarization of the fast shear wave, $\mathrm{S} \perp=\mathrm{S} 2$, the polarization of the slow shear wave (Rüger, 1996). 


\begin{tabular}{|c|c|c|c|c|}
\hline & Model 1 & Model 2 & Model 3 & Model 4 \\
\hline \multirow{4}{*}{ Layer 1 } & $\mathrm{Vp}=3.0 \mathrm{~km} / \mathrm{sec}$ & $\mathrm{Vp}=3.0 \mathrm{~km} / \mathrm{sec}$ & $\mathrm{Vp}=3.0 \mathrm{~km} / \mathrm{sec}$ & $\mathrm{Vp}=3.0 \mathrm{~km} / \mathrm{sec}$ \\
& $\mathrm{Vs}=1.5 \mathrm{~km} / \mathrm{sec}$ & $\mathrm{Vs}=1.5 \mathrm{~km} / \mathrm{sec}$ & $\mathrm{Vs}=1.5 \mathrm{~km} / \mathrm{sec}$ & $\mathrm{Vs}=1.5 \mathrm{~km} / \mathrm{sec}$ \\
& $\rho=2.00 \mathrm{~g} / \mathrm{cc}$ & $\rho=2.00 \mathrm{~g} / \mathrm{cc}$ & $\rho=2.00 \mathrm{~g} / \mathrm{cc}$ & $\rho=2.00 \mathrm{~g} / \mathrm{cc}$ \\
& $\mathrm{h}=2000 \mathrm{~m}$ & $\mathrm{~h}=2000 \mathrm{~m}$ & $\mathrm{~h}=2000 \mathrm{~m}$ & $\mathrm{~h}=2000 \mathrm{~m}$ \\
\hline \multirow{6}{*}{ Layer 2 } & $\mathrm{Vp}(0)=4.0 \mathrm{~km} / \mathrm{sec}$ & $\mathrm{Vp}(0)=4.0 \mathrm{~km} / \mathrm{sec}$ & $\mathrm{Vp}(0)=4.0 \mathrm{~km} / \mathrm{sec}$ & $\mathrm{Vp}(0)=4.0 \mathrm{~km} / \mathrm{sec}$ \\
& $\mathrm{Vs}_{1}(0)=2.0 \mathrm{~km} / \mathrm{sec}$ & $\mathrm{Vs}_{1}(0)=2.0 \mathrm{~km} / \mathrm{sec}$ & $\mathrm{Vs}_{1}(0)=2.0 \mathrm{~km} / \mathrm{sec}$ & $\mathrm{Vs}_{1}(0)=2.0 \mathrm{~km} / \mathrm{sec}$ \\
& $\epsilon=0.50$ & $\epsilon=0.50$ & $\epsilon=0.50$ & $\epsilon=0.01$ \\
& $\delta=0.10$ & $\delta=0.10$ & $\delta=0.10$ & $\delta=0.01$ \\
& $\gamma=0.02$ & $\gamma=0.05$ & $\gamma=0.10$ & $\gamma=0.01$ \\
& $\rho=2.2 \mathrm{~g} / \mathrm{cc}$ & $\rho=2.2 \mathrm{~g} / \mathrm{cc}$ & $\rho=2.2 \mathrm{~g} / \mathrm{cc}$ & $\rho=2.2 \mathrm{~g} / \mathrm{cc}$ \\
\hline
\end{tabular}

Table 2: Properties of anisotropic synthetic media, where $V_{P}$ is the compressional wave velocity, $V_{S}$ is the shear wave velocity. $V_{P}(0)$ and $V_{S 1}(0)$ are the vertically propagating $\mathrm{P}$-wave and $\mathrm{S} 1$ polarized shear wave velocities in the HTI azimuthally anisotrpic media, respectively. $\varepsilon, \delta$, and $\gamma$ are the exact Thomsen parameters of the equivalent HTI media. The only difference in the models 1, 2 and 3 is in the value of $\gamma$ in the lower layer. For Model 4, the anisotropy of the lower layer is only $1 \%$ essentially negligible. 


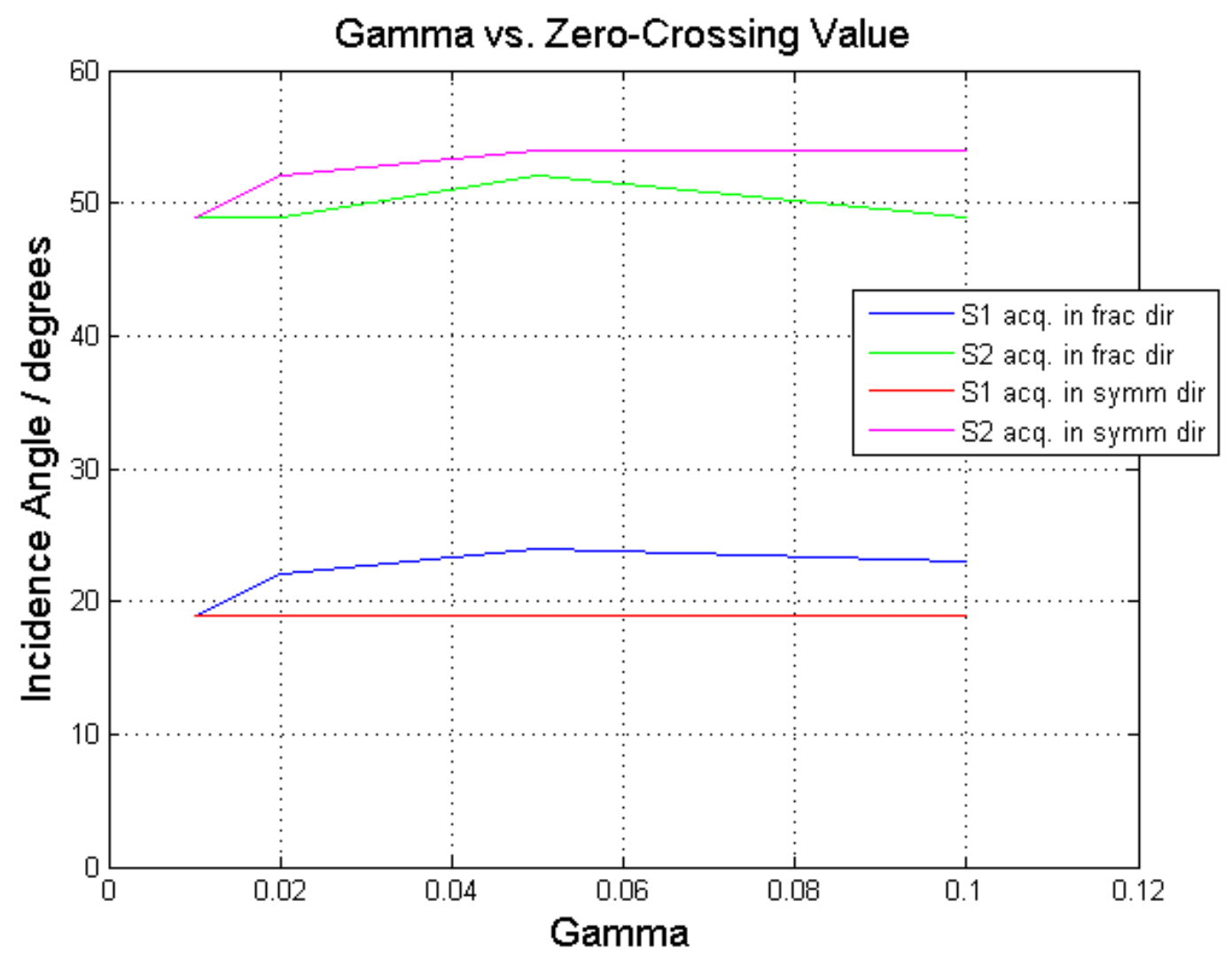

Figure 24: Values of the zero-crossing for SV-SV and SH-SH reflection coefficients as a function of incidence angle for two different models of an isotropic layer over an HTI medium. The layer properties are given in Table 2 and the graphs are calculated using Rüger's equation for isotropic over transversely isotropic (HTI) medium. Note zero crossings values for the various models which are used to identify singularities in the polarization correction analysis. In the top graphs the source is oriented parallel to fractures and in the lower plots the source is oriented perpendicular to fractures. Note similarity in the value for and SH-SH and SV-SV zero crossing values for a large range of $\gamma$ values. This is consistent with an almost constant value for zero-crossing for S1 and S2 polarizations for varying acquisition geometries. 


\section{SIMULATED 3D FIELD RECORDS FOR SEISMIC SHEAR SOURCES}

I demonstrate the process for simulating single shear-source position records with two horizontal source receiver components in a $3 \mathrm{D}$ survey for the case of an isotropic medium over an HTI anisotropic medium. These 3D shear-wave data are simulated using a Fourier frequency-wave number numerical modeling method (Mallick and Frazer, 1987). The parameters used to create the isotropic over anisotropic models are outlined in Table 3; various gamma values are chosen to test the effects of anisotropy (HTI) on the polarization distortion correction. The acquisition geometry of the isotropic over anisotropic modes is described in Figure 25. These simulations are the same geometry as those in Chapter 1, except the lower layer is azimuthally anisotropic. The examples in Chapter 1 used direct computations from Zoeppritz equations rather than to analytically compute the various trigonometric components. The maximum amplitude of each reflection observed in the model results is used for the polarization plots discussed here.

The lower layer fractured models are described as an HTI (horizontal transverse isotropy with a horizontal axis of symmetry) model, simulating vertical fractures. The HTI symmetry axes consistent with four fracture strike orientations are considered: 1) $\mathrm{N} 90^{\circ} \mathrm{E}$ or east-west strike orientation of simulated fractures in the map view 2) $\mathrm{N}^{\circ} 5^{\circ} \mathrm{E}, 3$ )

$\mathrm{N} 45^{\circ} \mathrm{W}$ and 4$) \mathrm{N} 30^{\circ} \mathrm{E}$. For computational convenience, the acquisition description is kept constant while the HTI medium is rotated around this constant acquisition geometry, so that the $x-y$ receiver orientation will be in radial $(\mathrm{SV})$ and transverse $(\mathrm{SH})$ orientations, respectively, for the individual element simulation. The SV-SV and SH-SH amplitude corrections are applied, and the corrected polarizations are calculated by the method used in the models shown in Figures 7 and 8 of Chapter 1. These corrections require knowledge of the SV-SV and SH-SH zero crossing, which were estimated by earlier 
modelling following Rüger (1998). The SV-SV and SH-SH zero crossings are $20^{\circ}$ and $50^{\circ}$, respectively.

The results for a horizontal source oriented in the east-west direction $\left(\mathrm{N} 90^{\circ} \mathrm{E}\right.$ orientation) are shown in Figure 26, and the results for the polarization corrections are shown in Figure 27. Note Figure 26 shows the counterpart to the example in Figure 7 of Chapter 1, but with an anisotropic lower layer. The isotropic models used for Figures 7 and 8 are different than the anisotropic models used here. Also note that the differences in polarity between the calculated data in Figure 7 and 8 of Chapter 1and the simulated reflection data considered here in Figures 26 - 31A. The computational results in Chapter 1 included the $180^{\circ}$ polarity reversal of the reflected shear waves at normal incidence. The amplitudes of reflections picked for the simulated reflections considered here did not compensate for this polarity reversal.

Figure 26 represents reflections from the top of an HTI medium with fractures oriented at E-W and anisotropy of $\gamma(\mathrm{v}) \sim 5 \%$ where $\gamma(\mathrm{v})$ is the Thomsen HTI azimuthal anisotropy parameter (Thomsen, 1986) defined with respect to the vertical plane as describe by Rüeger (2002). Comparison with Figure 7 (Chapter 1) shows many of the same polarizations characteristic as for the isotropic over isotropic, including a clear zero crossing and polarity change in the SV-SV reflectivity. At approximately $20^{\circ}$ incidence angle (the SV-SV zero crossing) the reflected S-wave is transverse to the source-receiver azimuth for essentially all source-receiver azimuths. At larger source-receiver offsets, the difference between the isotropic and HTI medium become more pronounced. Note the ellipse circled not the SV-SV zero crossing, but are of strong distortion in Figure 26 highlighting polarization distortion. This distortion is corrected remarkably well with the correction polarization applied (Figure 27). These results similar to those of Lyons (2006) 
thesis where they make reference to the $5 \%$ anisotropy where his correction also properly defines the polarization of the corrections.

The corrected data in Figure 27 are muted (polarizations shown as blue dots) in the zones where the SV-SV and SH-SH reflectivity is zero. This is to avoid noise introduced by a singularity in the correction at the zero crossing. Along the $\mathrm{x}$-axis, the SV-SV zero crossing is clearly observed as a polarity change about 1400 meters. In the $\mathrm{x}$ direction along the y-axis, only SH polarization (same as the source) is observed. The SH-SH zero crossing at an offset of 3400 meters, is observed only at the corners of the model in the $\mathrm{X}$ and $\mathrm{Y}$ axes for this plot. For the uncorrected polarizations (Figure 26), note that the observed polarizations along the $\mathrm{X}$ are predominantly in the direction of the source, which is also the fracture orientation. This particular example is for a $\gamma$ value of $5 \%$, relatively high anisotropy, so the preservation of the polarization in the source direction is identical to the fracture direction, is not surprising. As discussed earlier, large distortions in uncorrected polarization (Figure 26) at source-receiver azimuth at $45^{\circ}$, well beyond the SV-SV zero-crossing and near the SH-SH zero crossing is quite pronounced but is quite well corrected in Figure 27. Note source and fracture direction are similar to the results of (Lyons, 2006).

The results for a horizontal source oriented due east, $0^{\circ}$ orientation with respect to the x-axis with fracture direction $45^{\circ}$ east of north are shown in Figure 28, and the results for the polarization corrections are shown in Figure 29. This result, for the value of anisotropy $(\gamma=5 \%)$ shows near the orientation of anisotropy ( $45^{\circ}$ from the $y$, or north axis) is strong on the uncorrected data, (Figure 28) notably at offsets less than the SV-SV zero-crossing. In the corrected polarization (Figure 29) the corrected polarizations are dominantly in the direction of the HTI anisotropy, not the original source direction. This suggests that for even modest levels $(\gamma=5 \%)$ of azimuthal anisotropy in the lower layer 
(the upper layer is isotropic), the subsurface anisotropy resultant is imposed on the reflection anisotropy. This is consistent with the results of Lyons (2006).

Do note that in Figure 28 the reflected polarizations and the characteristic polarizations features seen in Figures 26 and 27 appear to be rotated about $45^{\circ}$ counterclockwise, consistent with the anisotropic character of the lower layer. The physical locations of the SV-SV nulls also have moved $45^{\circ}$ counter-clockwise. This is a reflected polarization equal to the anisotropy direction ( $45^{\circ}$ orientations). Figure 26 has the same features as Figure 28, again rotated $45^{\circ}$ counter-clockwise. In this case, the corrected plots (Figures 27 and 29) dominate the amplitudes for the plot of corrected reflected polarization.

Note that there are small changes in the length of the arrows (Figures 26, 27, 28 and 29) actually coinciding with the input polarization, consistent with the anisotropic character of the lower layer. Hence it appears to make no difference which way the source is oriented, the observed and corrected polarizations are all correctly oriented in the direction of the lower layer's anisotropy for $\gamma \geq 2 \%$.

The results for a source oriented in the north-south direction are shown in Figure 30.A and the results for the polarization correction are shown in Figure 30B. with varying value of gamma $(\gamma)$. Figure 30.A shows the result of a smaller anisotropy $(\gamma=2 \%)$ with an anisotropic lower layer. Polarizations at each receiver location again are calculated using simulation software. This is an HTI medium, with fractures oriented at $\mathrm{N} 45^{\circ} \mathrm{W}$, and anisotropy of $\gamma(\mathrm{v}) \sim 2 \%$ where $\gamma(\mathrm{v})$ is the Thomsen HTI anisotropy parameter (Thomsen, 1986) defined with respect to the vertical as describe by Rüeger (2002). The horizontal source is polarized in the N-S orientations and many of the same observations can be made, including the offset at which SV-SV reflectivity is zero. At approximately $20^{\circ}$ incidence angle (the SV-SV zero crossing) the reflected S-wave is transverse to the 
source-receiver azimuth for most azimuths consisted with the "polarizing" effect associated with the SV-SV zero - crossing. The point at which SH-SH reflectivity is zero is shown in Figure 30.A as a dashed circle at the corners of Figure 30A. Unlike the isotropic example the effective polarization of the S-wave is no longer consistently inline with the source-receiver azimuth as a result of being solely SV energy, but neither is it consistently inline with the fracture symmetry of $45^{\circ}$.

The results for a horizontal source due east with fracture orientation direction $\left(0^{\circ}\right.$ orientation) relative to an $\mathrm{X}$ oriented horizontal source are shown in Figure 31.A, and the results for the polarization corrections are shown in Figure 31.B. This is an HTI medium with fractures oriented at E-W and rather large azimuthal anisotropy of $\gamma(\mathrm{v}) \sim 10 \%$, where $\gamma(\mathrm{v})$ is the Thomsen HTI anisotropy parameter (Thomsen, 1986), consistent with respect to the vertical as describe by Rüger (2002). At approximately $20^{\circ}$ incidence angle (the "polarization angle", or the SV-SV zero crossing) the reflected S-wave is transverse to the source-receiver azimuth for most azimuths. The effect is not as pronounced as in the isotropic example because the anisotropy of the medium is now being imprinted on the polarization of the S-wave reflection process. Note that in this particular example is for a $\gamma$ value of $10 \%$, relatively high anisotropy, so the preservation of the polarization in the source direction is identical to the fracture direction, is not surprising. The large distortions in polarization at source-receiver azimuth at $45^{\circ}$, well beyond the $\mathrm{SV}-\mathrm{SV}$ zero-crossing and near the SH-SH zero crossing is also quite pronounced.

As discussed by Lyons (2006), by using a lower layer with HTI anisotropy with "fracture strike" oriented $45^{\circ}$ from the horizontal source polarization the correction decimates the amplitude for the corrected reflected polarizations. It appeared to Lyons (2006) that it makes no difference which way the source is oriented for $\gamma=5 \%$, such that 
the observed and corrected polarizations are all correctly oriented in the direction of the lower layer anisotropy.

We must take the similar $\gamma$ of $10 \%$ (see Figure $31 \mathrm{C}$ ) but we rotate the lower lying HTI geometry to fracture strike of $\mathrm{N} 30^{\circ} \mathrm{E}$ (northeast to southwest) defined with respect to the vertical as describe by Rüeger (2002). The point at which the SV-SV and SH-SH reflectivity is zero is muted (blue dots) in the plot (Figure 31D) of the corrected polarizations. Note in Figure 31D there are regions at offsets approaching the $\mathrm{SH}-\mathrm{SH}$ zero crossing that are not corrected properly corrected, but do follow define the fracture strike direction and is consistent with underlying HTI anisotropy. We do observe that at high values of $\gamma>10 \%$ the reflected polarization is properly defined by the corrected polarization data at all source-receiver azimuths and offset out to the SV-SV zero crossing.

After applying several simulations to different synthetic models for gamma values greater than $2 \%$ but less than $10 \%$ (Figure 30) it appears to make no difference which way the source is oriented, for source-receiver offsets less than the SV-SV zero crossing, the corrected polarizations are all properly oriented in the direction of the lower layer's HTI character. The orientation of Figures 30A, 31A and 31C show the same orientation of the vectors and zero crossing values. It can also be stated that at large offsets (greater than 1500 meters) the observed polarization is very different before the correction is applied. For larger values of anisotropy (10\%) it appears that uncorrected data properly defines the orientation of the anisotropy - so no correction would be required with these high levels (Figure 31). However for the low anisotropy of $1 \%$, the observed and corrected polarizations have no real differences from the simple isotropic over isotropic case because shear wave splitting seems to not occur. 
Gumble et al, (2006) suggests that in an isotropic over anisotropic case, the lower layer anisotropy will imprint a polarization on the reflection. This is confirmed by his results for models with anisotropy $>5 \%$ where the reflected polarization take on the orientation of the lower layers anisotropy. Lyons2006, Gumble 2006 and Campbell and Tatham 2012 all note that the anisotropic effect dominates over the reflection effect at higher degrees of anisotropy. Thus at higher percentages of anisotropy, the resultant reflected polarization map is the same no matter if the source is oriented in the eat to north direction. 


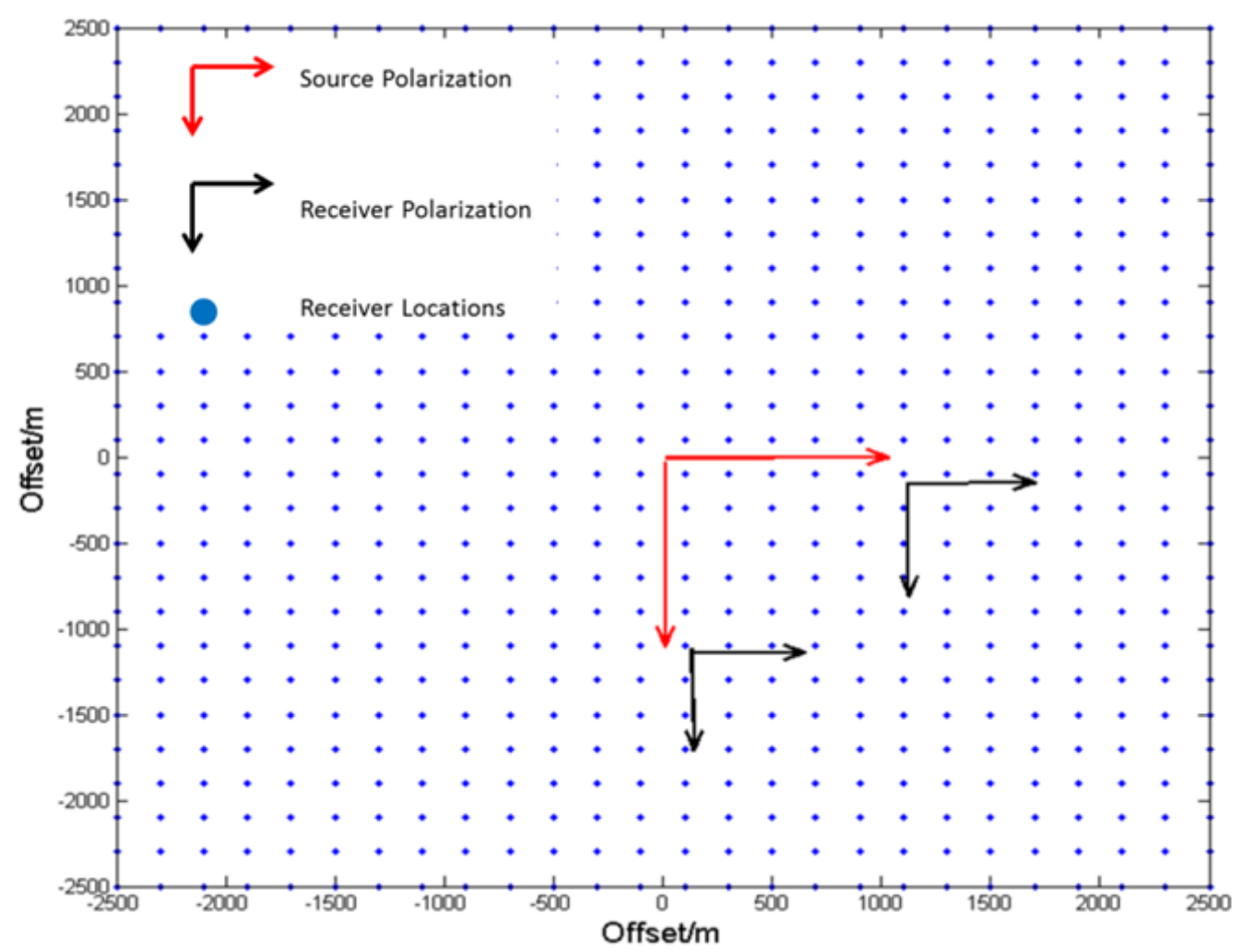

Figure 25: Acquisition geometry of the synthetic models. The two horizontal sources are situated at the center of the receiver positions. Receivers are positioned every $100 \mathrm{~m}$ in both $\mathrm{X}$ and $\mathrm{Y}$ directions with the source located at $(0,0)$. The black arrows indicate the N-S and E-W receiver orientations and the red arrows indicate N-S and E-W source orientations. Each shot has 625 pairs of observer N-S and E-W receiver traces. Each of the 625 receiver location can be resolved into a recorded shear-traces polarization and amplitude for each of the two source polarizations. 


\begin{tabular}{|c|c|}
\hline & Anisotropic Model \\
\hline Layer1 & $\mathrm{Vp}=3.0 \mathrm{~km} / \mathrm{sec}$ \\
$\mathrm{V} s=1.5 \mathrm{~km} / \mathrm{sec}$ \\
$\rho=2.00 \mathrm{~g} / \mathrm{cc}$ \\
$\mathrm{h}=2000 \mathrm{~m}$ \\
\hline (Isotropic) & $\mathrm{Vp}(0)=4.0 \mathrm{~km} / \mathrm{sec}$ \\
& $\mathrm{Vs}(0)=2.0 \mathrm{~km} / \mathrm{sec}$ \\
$\epsilon=0.50$ \\
$\delta=0.10$ \\
(Anisotropic) \\
$\gamma=0.02-0.1($ variable $)$ \\
\\
Fracture Strike $=$ East \\
$\rho=2.2 \mathrm{~g} / \mathrm{cc}$ \\
$\mathrm{h}-$ half-space \\
\hline
\end{tabular}

Table 3: Properties of anisotropic (transversely isotropic) synthetic media, where $\mathrm{Vp}$ is the compressional wave velocity, Vs is the shear wave velocity. Vp (0) and Vs (0) are the vertically propagating p-wave and s-wave velocities in the HTI media, respectively. $\varepsilon, \delta$, and $\gamma$ are the exact Thomsen parameters of the equivalent HTI media. A range of $\gamma$ values are used to test its effect on the reflected waves. 


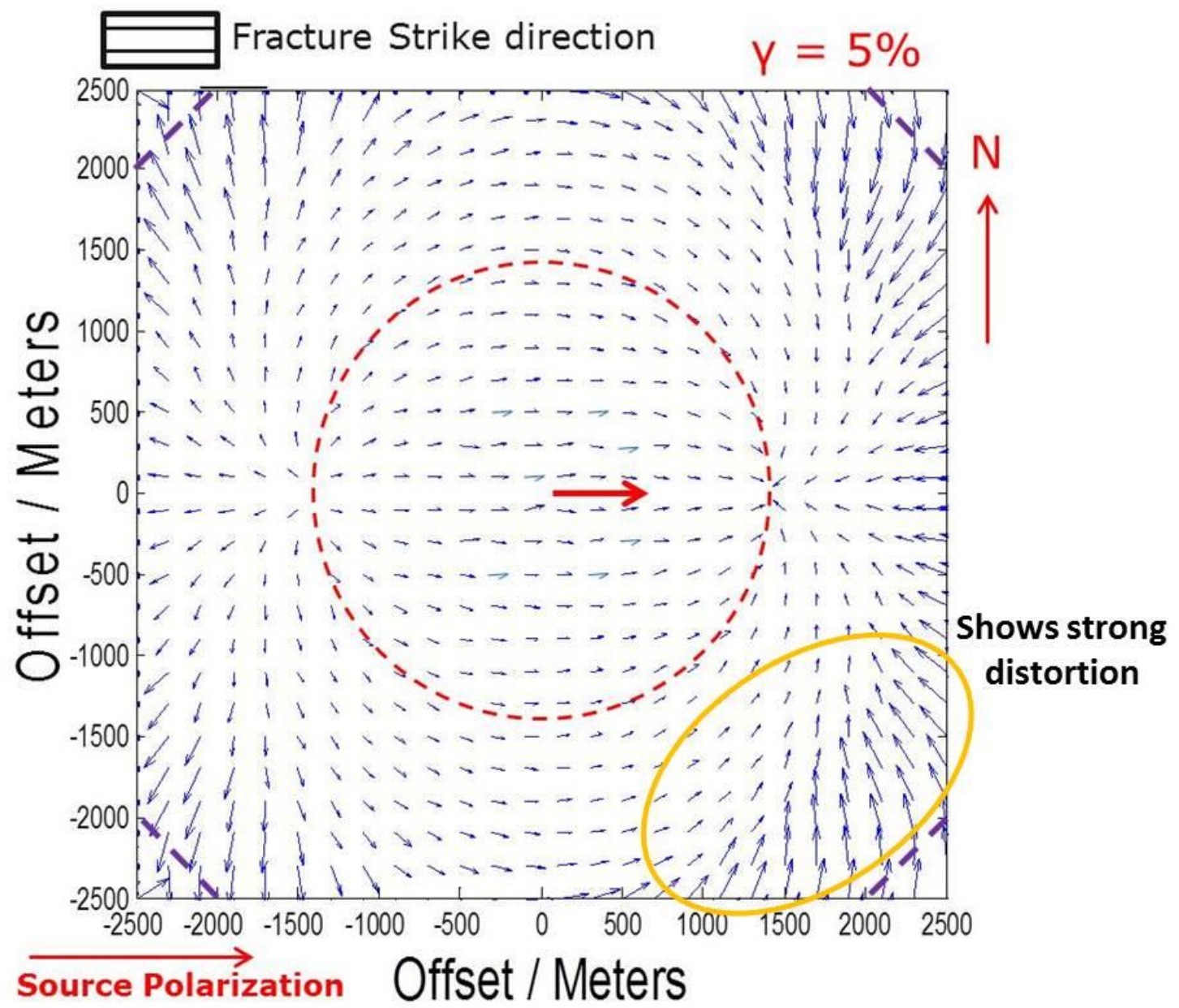

Figure 26: Observed (simulated) polarizations in a single source record (X-oriented source at center) of a 3D survey (map view) for a reflector depth of $2000 \mathrm{~m}$. HTI fracture orientation in the lower medium is E-W $\left(90^{\circ}\right.$ west of north). The circle indicated the offset corresponding to an incidence angle of $22^{\circ}$ (the SV-SV zero crossing) at an offset of about $1400 \mathrm{~m}$. The $50^{\circ} \mathrm{SH}-\mathrm{SH}$ zero crossing is at $2400 \mathrm{~m}$. The length of the vectors indicates the amplitude of the recorded data, and the orientation of the vectors indicates the observed polarization. Thomsen parameters used are; Gamma $=0.05$, Delta $=0.10$, Epsilon $=0.30$. The upper layer is isotropic. Note consistent E-W polarization of the reflected shear-waves at offsets up to at least 500 meters, and almost to the SV-SV zero crossing. Also note the rather strong distortion of polarization just beyond the SV-SV zero crossing, especially source-receiver azimuths at $45^{\circ}$ (ellipse), including the high amplitude and strong distortion at about 1500 meters well before the $\mathrm{SH}-\mathrm{SH}$ zero crossing. 


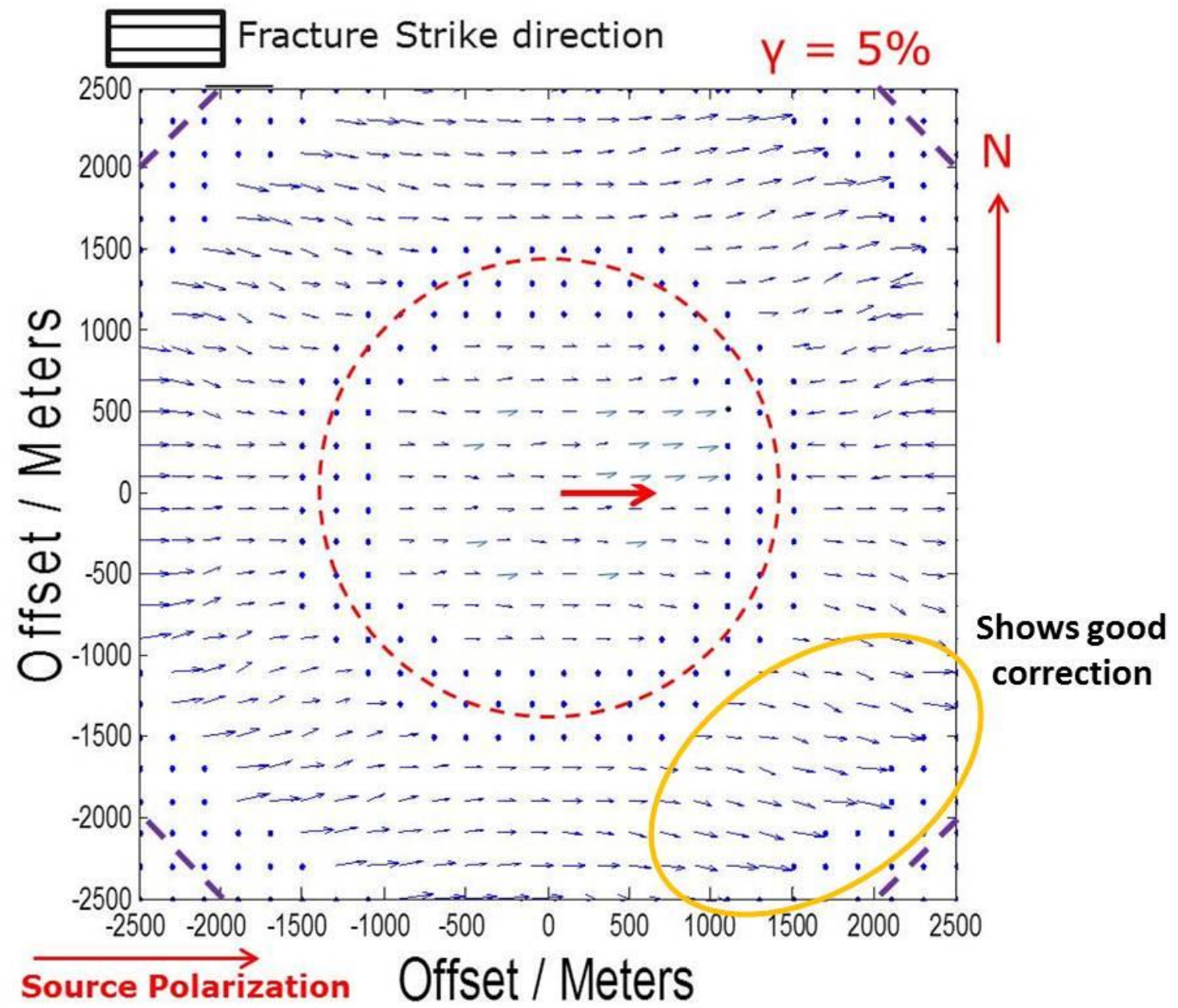

Figure 27: Polarization correction applied to the simulated polarizations (Figure 26) in a single 3D source 3D record (X-oriented source at center) of a 3D survey (map view) for a reflector depth of $2000 \mathrm{~m}$. The circle indicated the offset corresponding to an incidence angle of $22^{\circ}$ (the SV-SV zero crossing). The length of the vectors indicates the amplitude of the recorded data, and the orientation of the vectors indicates the observed polarization. Fractures are oriented east - west. Thomsen parameters used are; Gamma $=0.05$, Delta $=0.10$, Epsilon $=0.30$. The SH-SH zero crossing is at 2400 meters. Note the consistency of the corrected polarization, other than in SV-SV zerocrossing gap, out to 1400 meters and beyond. Now we see below the SV-SV zero-crossing all the reflected polarizations are correctly oriented. Beyond the SV-SV zero crossing (ellipse) the reflected polarizations are also correctly define the lower lying anisotropy. 


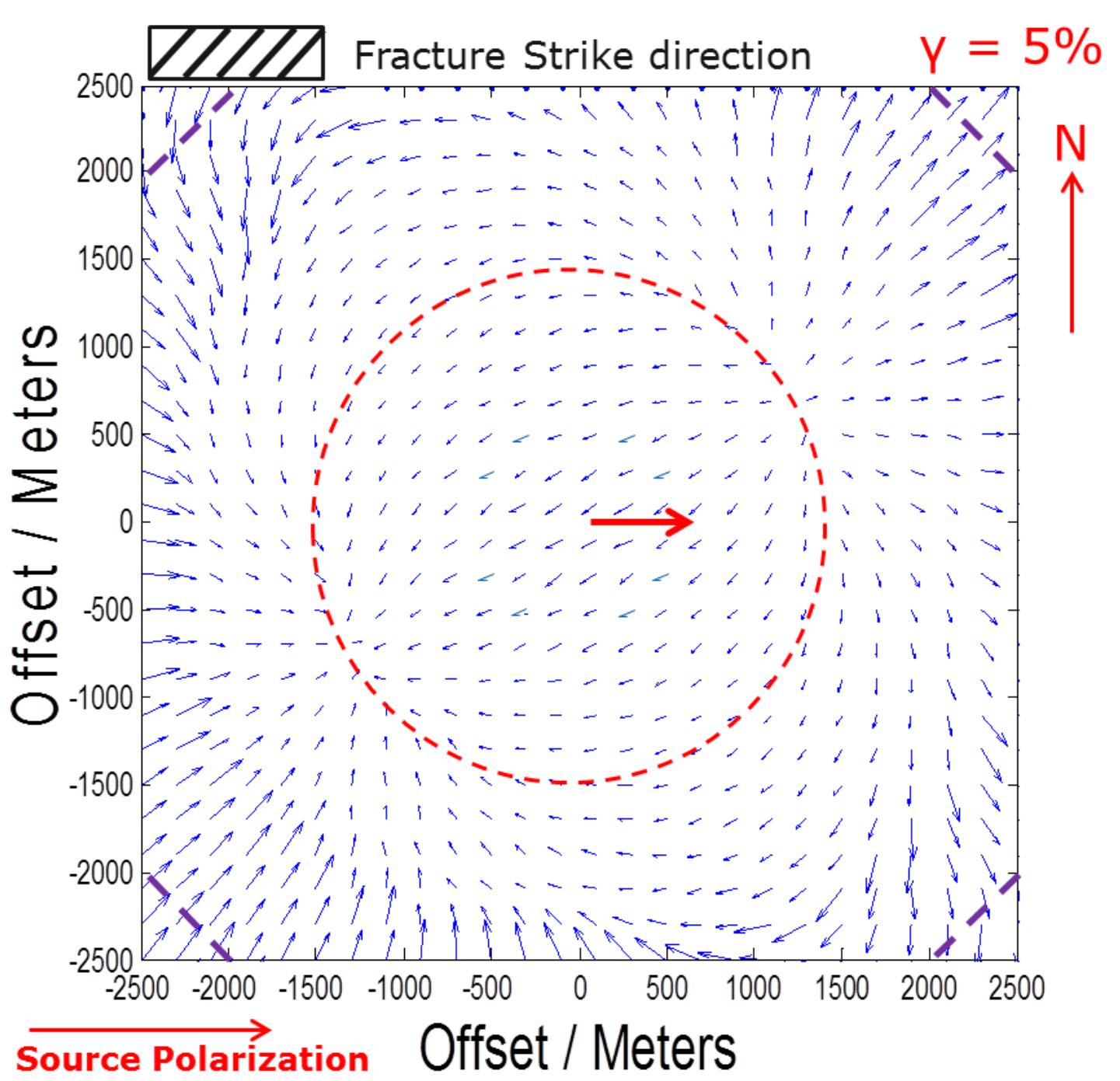

Figure 28: Observed (simulated) polarizations for the same source and receiver geometry and medium properties as Figure 27 but the fractures are oriented $45^{\circ}$ east of north. Note the consistency of the vectors at the fracture strike direction of $45^{\circ}$. This is consistent with the $\mathrm{S} 1$ polarization of the lower layer. This polarization is observed at zero-offset, suggesting that the lower medium controls the direction of polarization. Beyond the SV-SV zero-crossing at $1400 \mathrm{~m}$, the polarizations become quite different and quite pronounced beyond the SH-SH zero-crossing. Thomsen parameters used are; Gamma = 0.05 , Delta $=0.10$, Epsilon $=0.30$. Note the observed (simulated) polarizations in a single $3 \mathrm{D}$ source record (X-oriented source at center) of a 3D survey (map view) for a reflector depth of $2000 \mathrm{~m}$. The circle $(1400 \mathrm{~m})$ indicated the offset corresponding to an incidence angle of $22^{\circ}$ (the SV-SV zero crossing). The purple circle shows the SH-SH zero crossing at $2400 \mathrm{~m}$. 


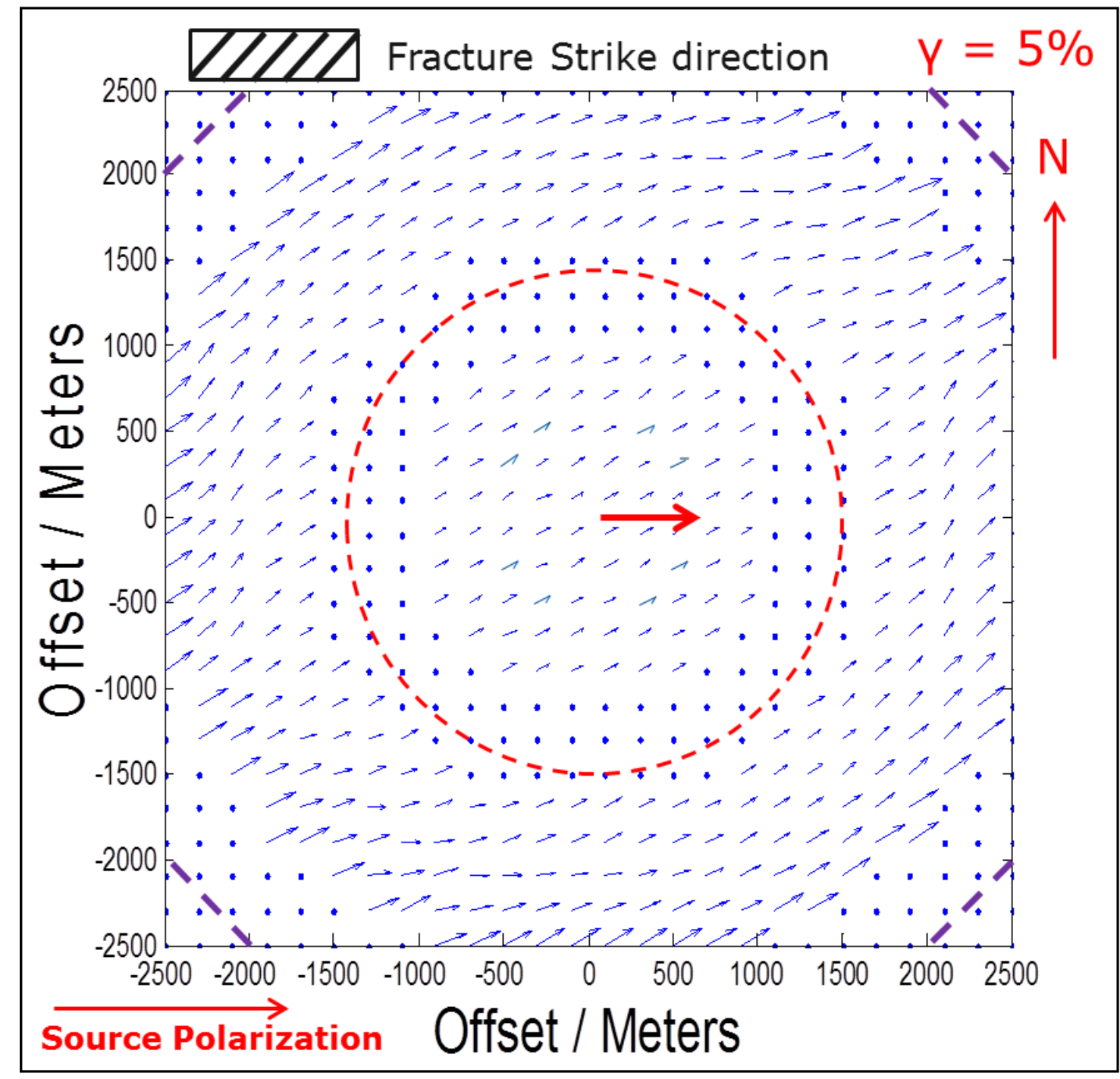

Figure 29: Polarization correction applied to Figure 29. Note the resultant vector arrows are oriented with the same direction as the medium anisotropy for a single $3 \mathrm{D}$ source record (X-oriented source at center). The correction results in the orientation with substrate fracture direction rather than the source direction. The correction loos great all the way to the SH-SH zero crossing but the correct fracture orientation rather than the source direction (as in the isotropic case). Reflector depth is at $2000 \mathrm{~m}$. The circle indicated the offset corresponding to an incidence angle of $22^{\circ}$ (the SV-SV zero crossing). The length of the vectors indicates the amplitude of the recorded data, and the orientation of the vectors indicates the observed polarization. Fractures are oriented $\mathrm{N} 45^{\circ} \mathrm{E}$ ( northeast - southwest). Thomsen parameters used are; Gamma $=0.05$, Delta $=0.10$, Epsilon $=0.30$. 


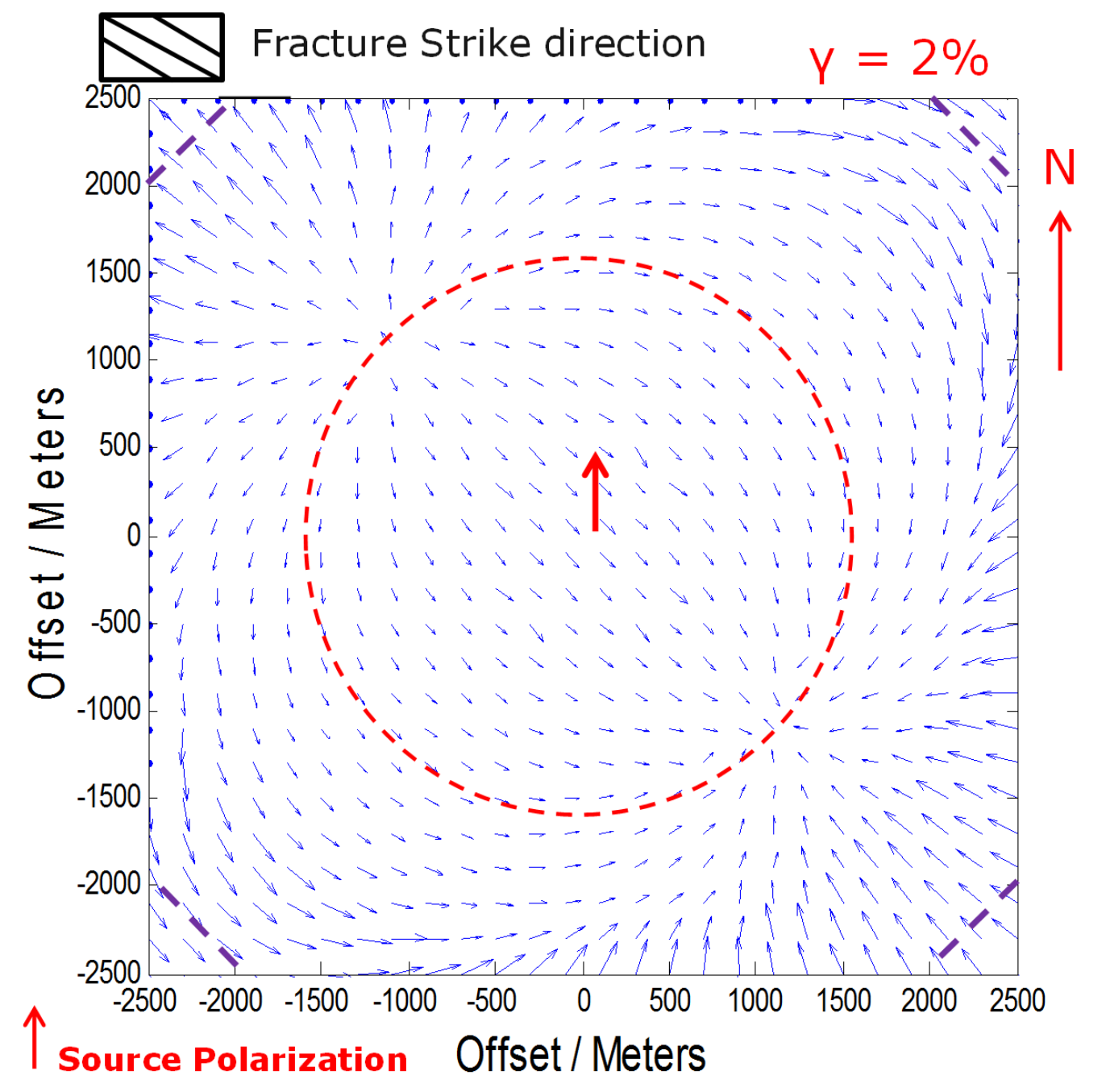

Figure 30.A: Simulated data for same acquisition geometry as Figure 27 and 29 but for decreased anisotropy $(\gamma=2 \%)$, a north $(Y)$ oriented horizontal source and HTI (fracture) strike of $\mathrm{N}^{\circ} 5^{\circ} \mathrm{W}$. Note the observed (simulated) polarizations in a single $3 \mathrm{D}$ source record ( $\mathrm{Y}$-oriented source at center) of a 3D survey (map view). Note the change in the polarization direction from Figure 28 the input source polarizations are different which causes the reflected polarization direction to change but still properly define the HTI anisotropy. The reflected polarization defines the underlying anisotropy at offsets less than the SV-SV zero crossing. The circle indicated the offset corresponding to an incidence angle of $22^{\circ}$ (the SV-SV zero crossing). The length of the vectors indicates the amplitude of the recorded data, and the orientation of the vectors indicates the observed polarization. Fractures are oriented $\mathrm{N} 45^{\circ} \mathrm{W}$ (northwest - southeast). Thomsen parameters used are; Gamma $=$ 0.02 , Delta $=0.10$, Epsilon $=0.30$. 


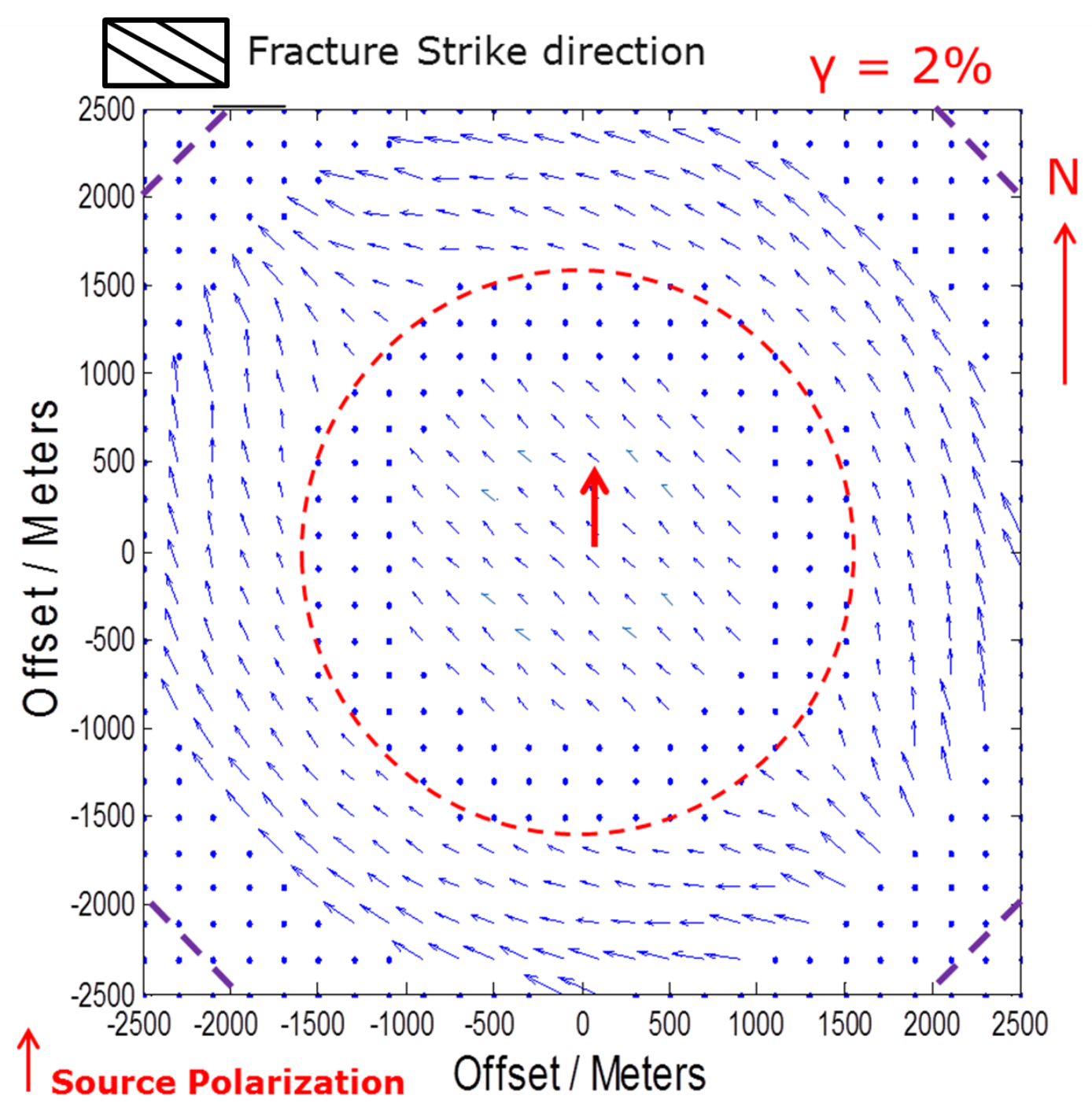

Figure 30.B: Polarization correction applied to Figure 30A. We can see it also has the correct fracture direction at offset up to at least the SV-SV zero crossing. Note we muted values around the zero crossing due to the singularity. The reflected polarizations correctly define the lower layer anisotropy even slightly beyond the SV-SV zero crossing. We had to mute out several values before the SH-SH zero crossing because the values were quite large in comparison to the other polarization values. The circle indicated the offset corresponding to an incidence angle of $22^{\circ}$ (the SV-SV zero crossing). The length of the vectors indicates the amplitude of the recorded data, and the orientation of the vectors indicates the observed polarization. Fractures are oriented $\mathrm{N} 45^{\circ} \mathrm{W}$ (northwest - southeast). Thomsen parameters used are; Gamma $=0.02$, Delta $=0.10$, Epsilon $=0.30$. 


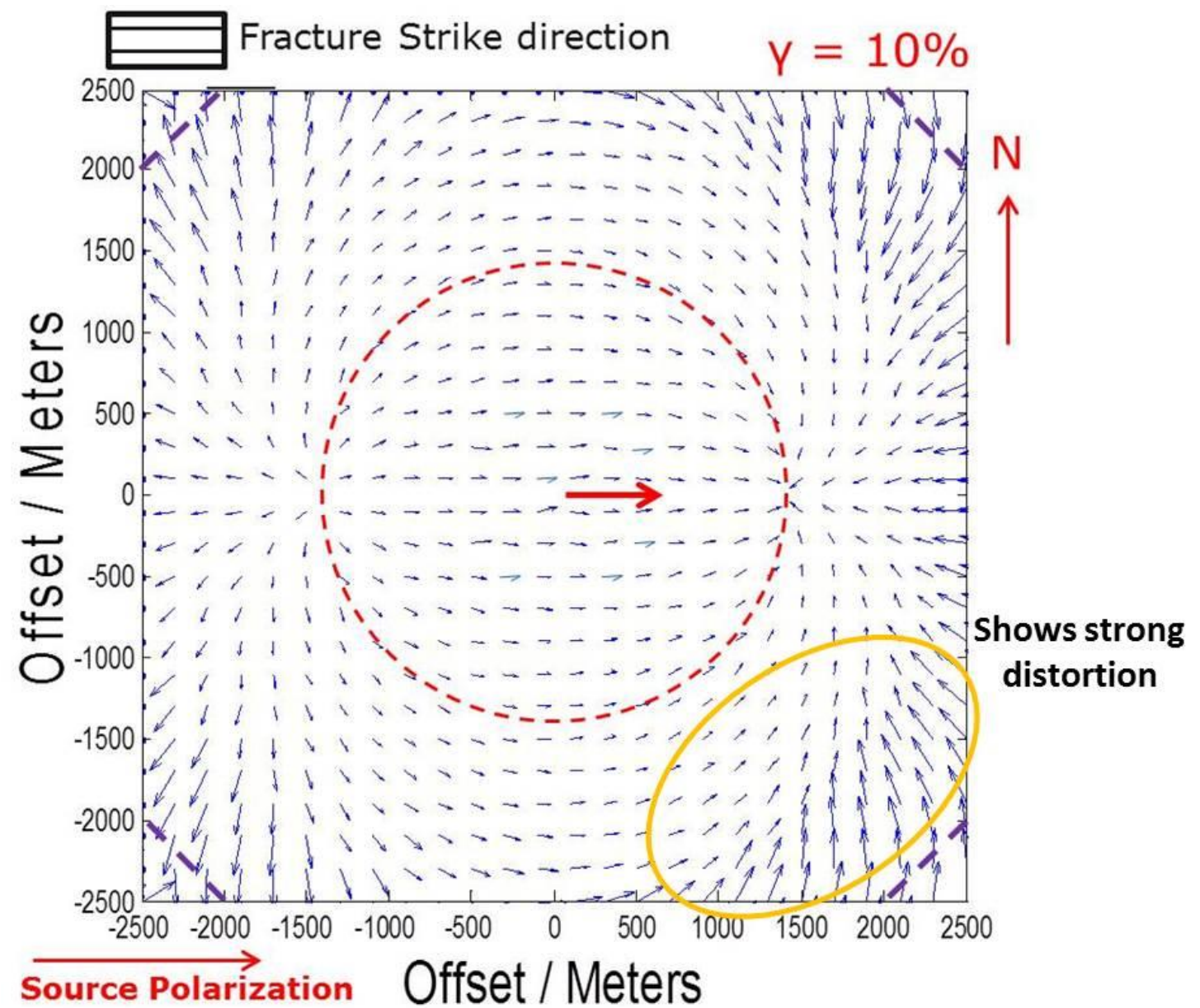

Figure 31.A: Figure used to simulate a $10 \%$ gamma anisotropic medium and it was observed that no matter the source orientation the observed polarization is properly oriented. This mimics observed (simulated) polarizations in a single 3D source record (X-oriented source at center) of a 3D survey (map view) for a reflector depth of $2000 \mathrm{~m}$. The circle indicated the offset corresponding to an incidence angle of $22^{\circ}$ (the SV-SV zero crossing). Note the zero crossing value at an $\mathrm{X}$ offset of 1350 meters corresponding to $22^{\circ}$. The length of the vectors indicates the amplitude of the recorded data, and the orientation of the vectors indicates the observed polarization. Fractures are oriented east-west. Thomsen parameters used are; Gamma $=0.10$, Delta $=0.10$, Epsilon $=0.30$. 


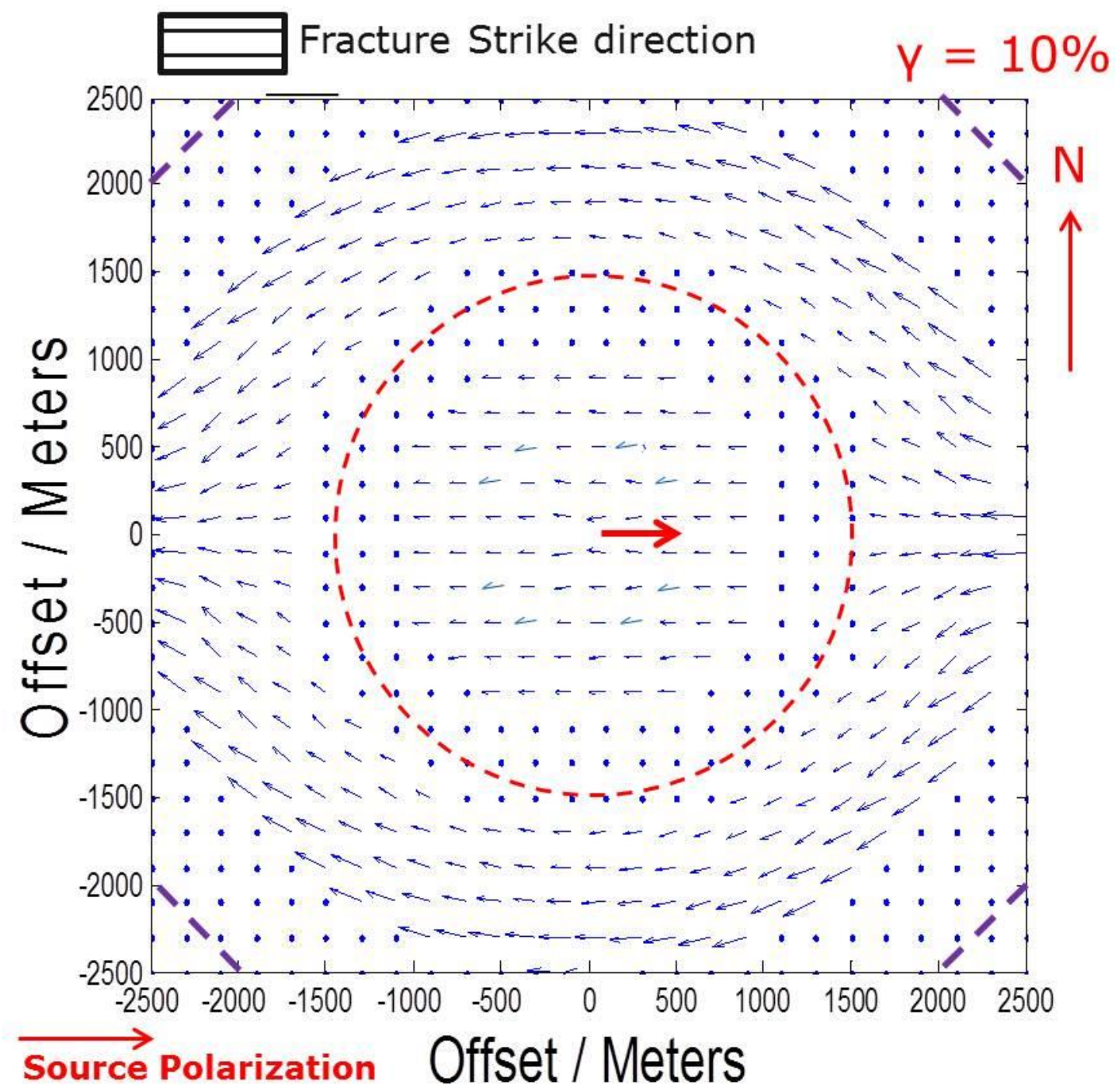

Figure 31.B: Polarization correction applied toFigure $32 \mathrm{~A}$ where the corrected polarization applied to the simulated data in a single 3D source record ( $\mathrm{Y}$ oriented source at center) of a 3D survey (map view) for a reflector depth of $2000 \mathrm{~m}$. The circle indicated the offset corresponding to an incidence angle of $22^{\circ}$ (the SV-SV zero crossing). Note the observed polarization is corrected oriented before and after the polarization correction is applied. So it can be said no matter the source orientation once gamma is greater than $10 \%$ we can define fracture strike. The length of the vectors indicates the amplitude of the recorded data, and the orientation of the vectors indicates the observed polarization. Fractures are oriented east - west. Thomsen parameters used are; Gamma $=0.10$, Delta $=0.10$, Epsilon $=0.30$. 


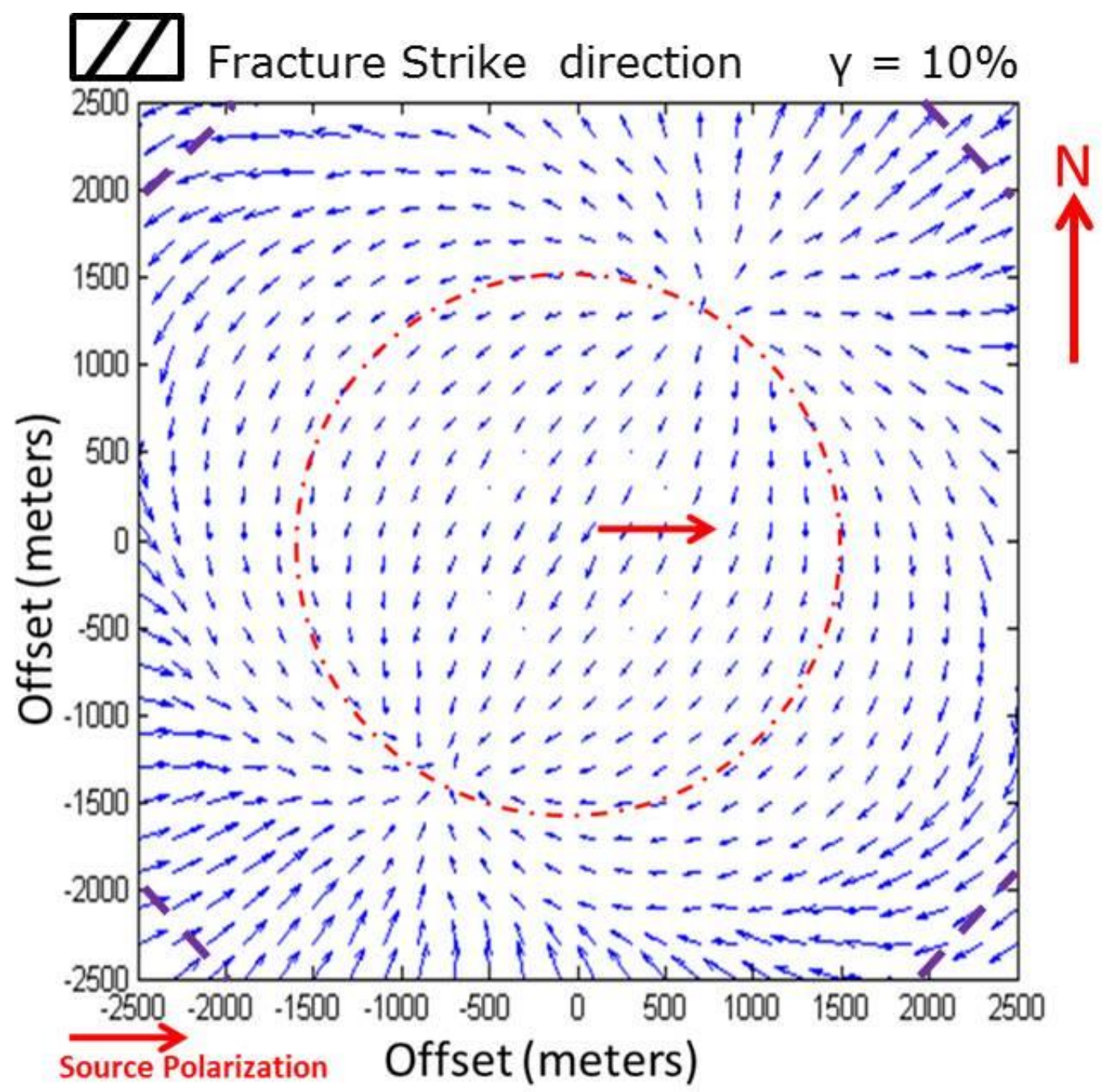

Figure 31.C: Figure used to simulate a $10 \%$ gamma anisotropic medium and it was observed that no matter the source orientation the observed polarization is properly oriented. It mimics observed (simulated) polarizations in a single 3D source record (X-oriented source at center) of a 3D survey (map view) for a reflector depth of $2000 \mathrm{~m}$. The circle indicated the offset corresponding to an incidence angle of $22^{\circ}$ (the SV-SV zero crossing). Note the zero crossing value at a $\mathrm{X}$ offset of 1250 meters corresponding to $22^{\circ}$. The length of the vectors indicates the amplitude of the recorded data, and the orientation of the vectors indicates the observed polarization. Fractures are oriented $\mathrm{N} 30^{\circ} \mathrm{E}$ (northeast - southwest). Thomsen parameters used are; Gamma $=0.10$, Delta $=0.10$, Epsilon $=0.30$. Note the polarization correctly defines the lower layer anisotropy even beyond the SV-SV zero-crossing. 


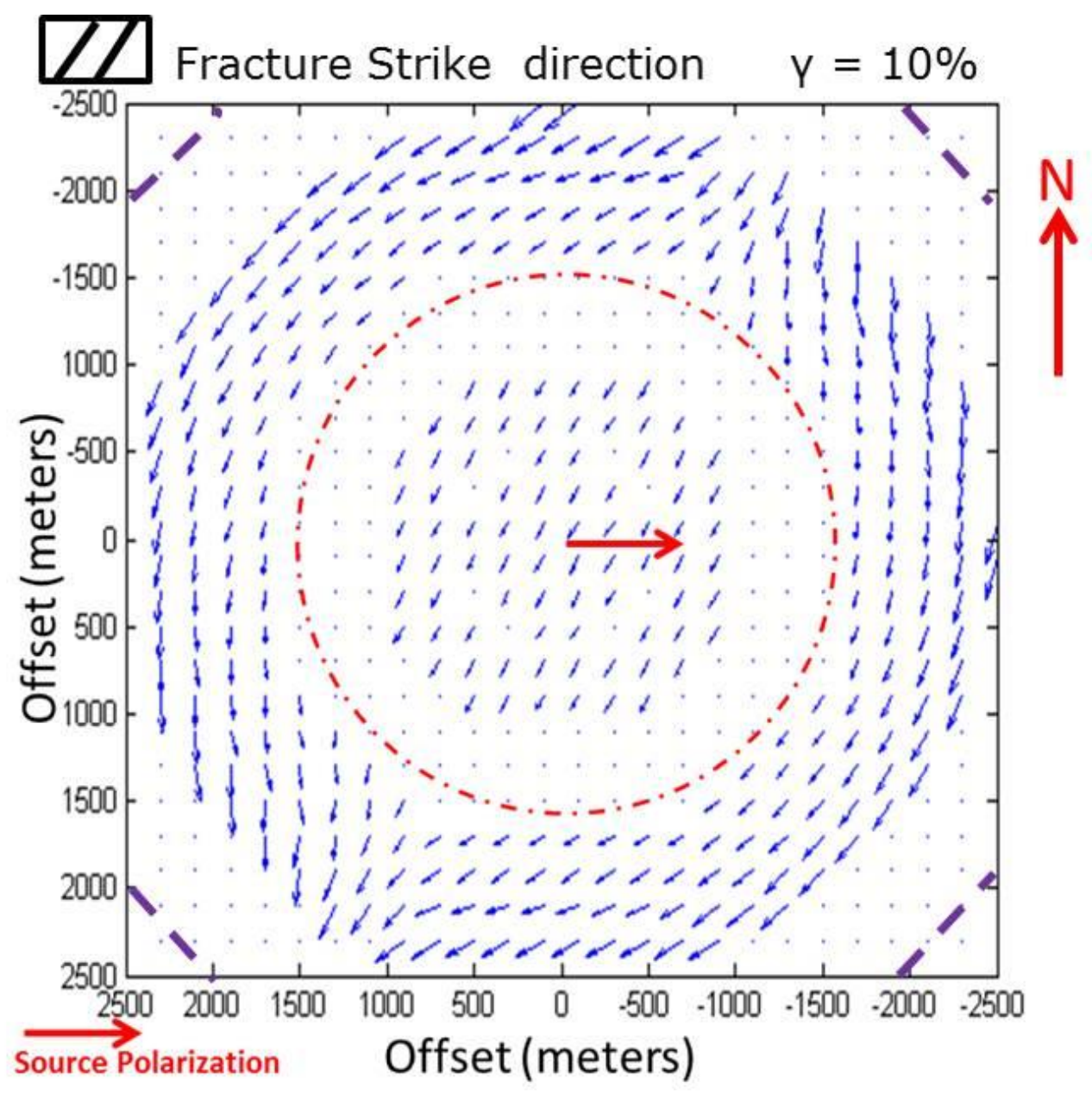

Figure 31.D: Corrected polarization plot from Figure $32 \mathrm{~A}$ where the corrected polarization applied to the simulated data in a single 3D source record (Yoriented source at center) of a 3D survey (map view) for a reflector depth of $2000 \mathrm{~m}$. The circle indicated the offset corresponding to an incidence angle of $22^{\circ}$ (the SV-SV zero crossing). The correction results in the orientation with substrate fracture direction rather than the source direction. The correction loos great all the way to the $\mathrm{SH}-\mathrm{SH}$ zero crossing but the correct fracture orientation rather than the source direction (as in the isotropic case). Note the observed polarization is corrected oriented before and after the polarization correction is applied. So it can be said no matter the source orientation once gamma is greater than $10 \%$ we can define fracture strike. The length of the vectors indicates the amplitude of the recorded data, and the orientation of the vectors indicates the observed polarization. Fractures are oriented $\mathrm{N} 30^{\circ} \mathrm{E}$ (northeast - southwest). Thomsen parameters used are; Gamma $=0.10$, Delta $=0.10$, Epsilon $=0.30$. 


\section{CHAPTER 3: BLACK BEAR SURVEY, ARDMORE BASIN OKLAHOMA, USA}

I expand the observation of polarization distortion in synthetic examples of direct shear-wave reflections examined in Chapter 2 to real field data recorded with direct shear-source from the Black Bear 3D 9C seismic survey. The Black Bear survey is located in Northeast corner of Stephens county Oklahoma in the Ardmore Basin. Tectonically the Ardmore basin lies in the foreland of the Ouachita fold and thrust belt and within the splays of the Washita Valley wrench system (Figure 32). The surface seismic data were gathered in 1998 by PGS land team and given The University of Texas at Austin's Bureau of Economic Geology (Exploration Geophysics Laboratory) and

graciously provided for this study. Surface areal coverage of the survey is $42 \mathrm{~km}^{2}$ (15 square miles). Subsurface borehole information from the published results (Beckham, 1996) of anisotropy in this tectonic region is interpreted from shear-wave polarizations a 9C VSP and dipole logs in the Texaco Brady well; about $30 \mathrm{~km}$ from the surface survey are also included. These borehole results are considered in the analysis of the surfacerecorded shear wave data.

Analysis techniques of the observed polarization distortion, for both the synthetic and surface data are discussed in Chapter 4. 


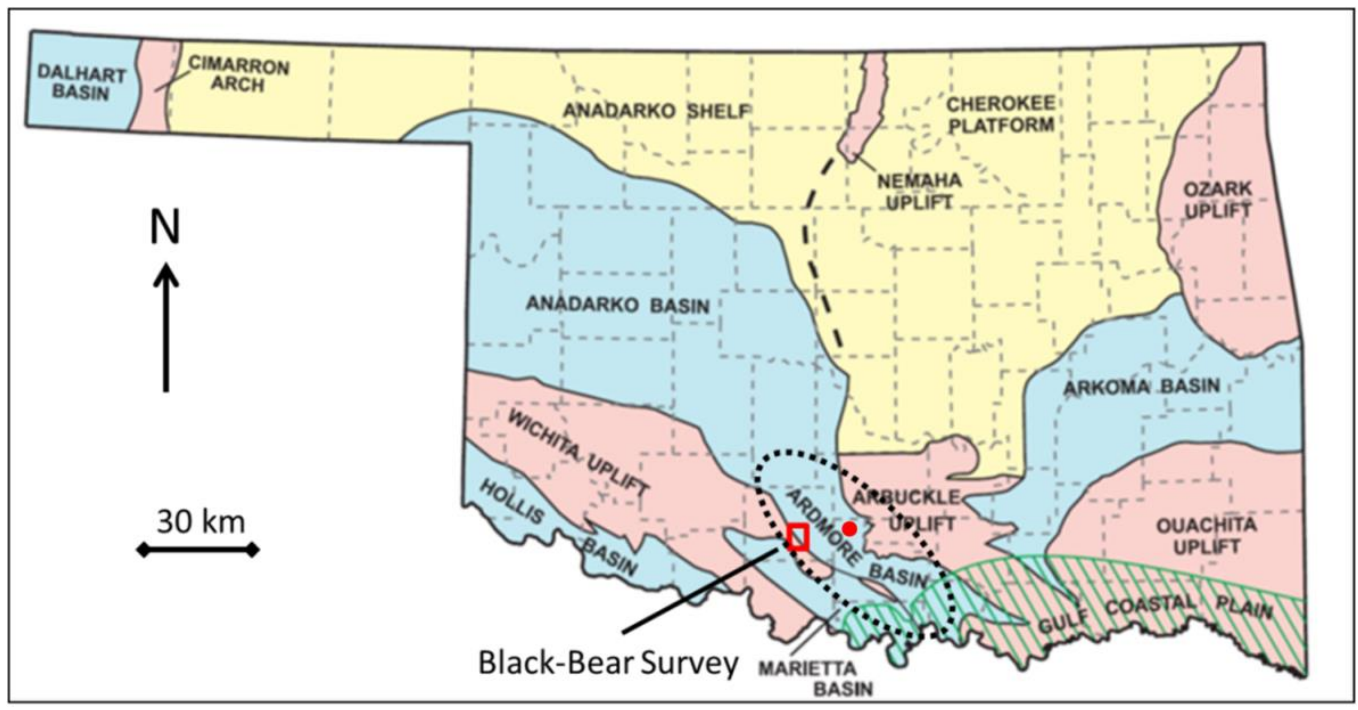

Figure 32: Generalized distribution of major geologic provinces of Oklahoma. The Ardmore Basin is in south-central Oklahoma, mostly in Marshall, Carter and eastern Stephens Counties. The red outline represents the location of the Black-Bear survey. The Texaco Brady 1 well is about $30 \mathrm{~km}$ east of the Black-Bear survey, shown by the red circle (modified from, Johnson, 2008).

\section{Early Geological History of the Ardmore Basin}

The 3D-9C surface seismic data to be examined were acquired near the Ardmore Basin of Oklahoma. The Ardmore Basin, together with the Marietta Basin to the south, the Anadarko Basin to the northwest and the adjacent uplifts, has a complex tectonic and depositional history. The most impressive aspects of this history are the extraordinary thicknesses of late Mississippian and Pennsylvanian sediments that accumulated in the basins and the complex folded and faulted nature of the rocks when compared to the surrounding areas. Further, there is a major unconformity between the Mississippian and Pennsylvanian sediments. This top of Mississippian unconformity extends over a large portion of North America. The geology of the area has its underpinnings in a much older geological period. The Southern Oklahoma basement rock is characterized by granites of 
age of about 550 million years (pre-Cambrian). The granite basement was formed along with a series of northwest- trending faults formed in the broad zone across southern and southwestern Oklahoma and extending to near Amarillo in the Texas panhandle. This tectonic geologic evolution is summarized in Figure 33 with the various structural developments from middle Cambrian to Permian age.

The Ardmore basin lies in the foreland of the Ouachita fold and thrust belt and within the splays of the Washita Valley wrench system. The Ardmore basin underwent initial subsidence in the latest Precambrian through Middle Cambrian as part of the southern Oklahoma aulacogen associated with the initial rifting and volcanic fill of the granitic basement (Fig 33A) (Hoffman et al., 1974). The aulacogen was oriented transverse to the early Paleozoic continental margin and became narrower toward the northwest. In the Late Cambrian through Pennsylvanian, basin subsidence took the form of a broad syncline. A change in tectonic setting occurred at the initiation of plate convergence in Late Mississippian to Middle Pennsylvanian time. Thus, there may be differences in the distribution of tectonic stresses applied during deposition of the Mississippian and Pennsylvanian sediments. During this episode, flysch sediments (figure 34D) were deposited in a foredeep north of the advancing late Mississippian Early Devonian Ouachita orogeny. Devonian Ouachita overthrusts adjacent to the Ardmore basin are transverse to the Washita alley wrench system and were active between latest Devonian to Middle Pennsylvanian (Ham and Wilson, 1967).

Strike slip on the Washita Valley faults was in part contemporaneous with deformation in the Ouachita orogeny and-occurred in the Early and Late Pennsylvanian (Ham and Wilson, 1967; Tanner, 1967; Haas, 1981). Wielchowsky and Gilbert (1982) proposed that the crustal collision that caused the Ouachita thrusting also caused the strike-slip faulting in the adjacent foreland. The splay pattern of the wrench system is 
thought to have been inherited from the earlier framework of the southern Oklahoma aulacogen (Haas, 1981). Evidence of wrench faulting in the foreland is quite compelling. The offset of Ordovician facies lines demonstrates $64 \mathrm{~km}$ (40 mi) of Early Pennsylvanian left slip on the main Washita Valley fault (Tanner, 1967). Splays to the south and north of this fault also have offset stratigraphic piercing points documenting left slip but of smaller magnitude. Haas (1981) inferred 16 to $24 \mathrm{~km}$ (10 to $15 \mathrm{mi})$ of left slip along the Reagan Fault (Figure 34) on the basis of stratigraphic and structural relationships. Secondary faults and folds associated with the Reagan Fault and several other faults in the system have patterns characteristic of wrench faults (Wilcox et al., 1973).

In addition, the regional tectonic folding, which provides many of the hydrocarbon traps, has a consistent en echelon pattern compatible with a left-slip deformation (Figure 34). The Ardmore basin wrench fault is a subsidiary element of the Washita Valley system. It has had a small left-lateral displacement. As production of gas is often controlled by fractures and faulting in the Oklahoma Basin it has long been a case study in fracture determination using geophysical means, including borehole sonic logs, VSP's, and multicomponent data (Mattocks, 1998). An investigation performed by Beckham (1996) also confirmed that fracture characterization is an important factor in the characterization of this sedimentary section. Interestingly, Beckham (1996) found that characterization and orientation of the fracture trends, as estimated by observed anisotropy in 9C VSP and dipole sonic logs, above and below the top of the Mississippian unconformity are markedly different. 


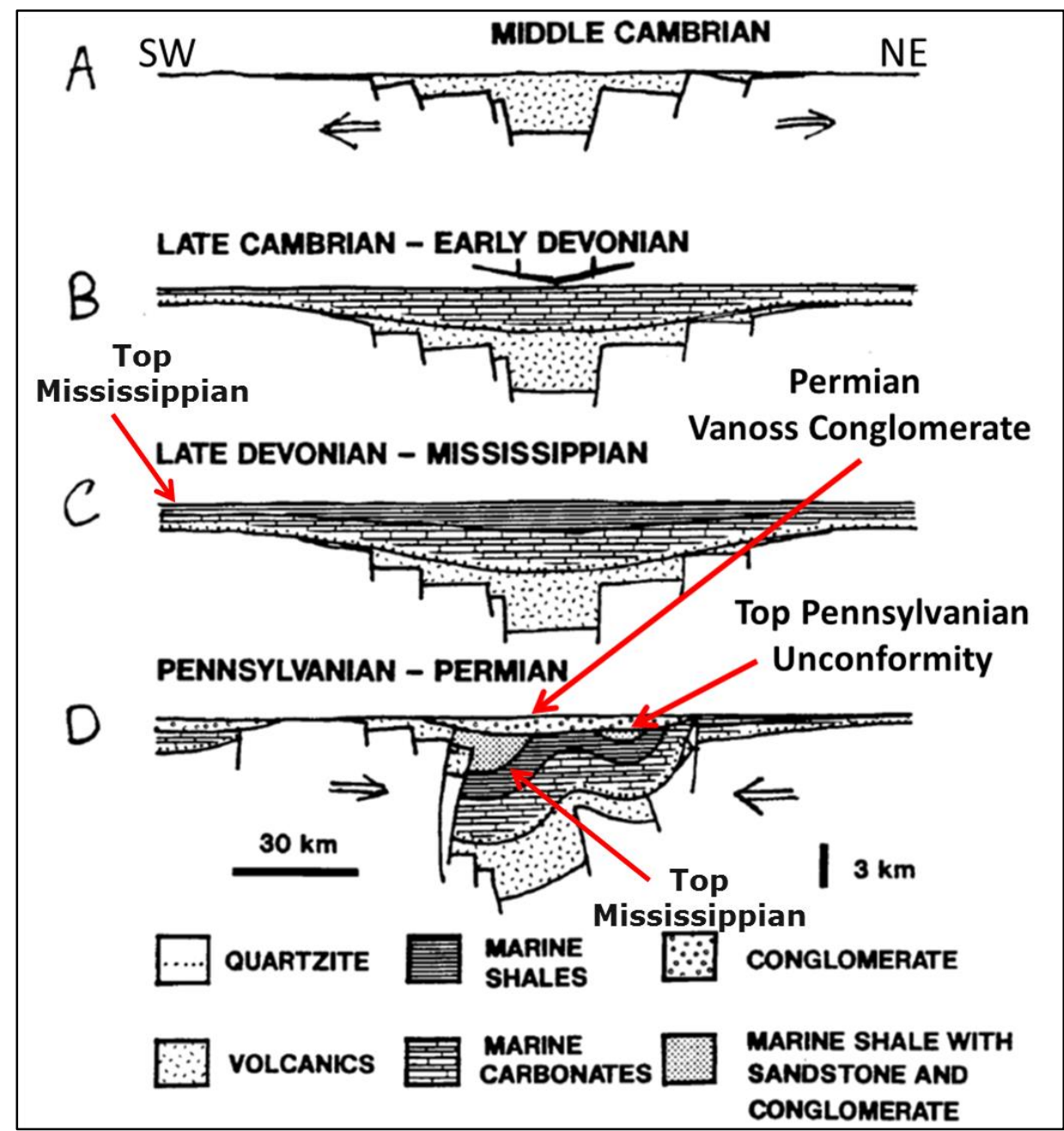

Figure 33: Structural development of the Southern Oklahoma aulacogen source. A: Middle Cambrian extension, faulting (rifting) of the granite basement and filling of rift with volcanic rocks. B: Late Cambrian to Early Devonian subsidence and accumulation of mostly marine limestone and to a lesser extent sandstone and shale. C: Continued subsidence in the Late Devonian to Late Mississippian and deposition of mostly marine shale and minor sandstone and limestone. D: Folding and faulting during Wichita and Arbuckle orogenies during Pennsylvanian and Permian time. Note the top of the local Pennsylvanian angular unconformity at the base of the conglomerate that formed as a result of the Arbuckle orogeny (Kleehammer, 1991). The top of the regional Mississippian unconformity occurs below the top of the Pennsylvanian an in this section is in very complex folded region. The top of the Mississippian is observable on the seismic section. The Top of the Mississippian unconformity is regional across North America (including Alberta) 


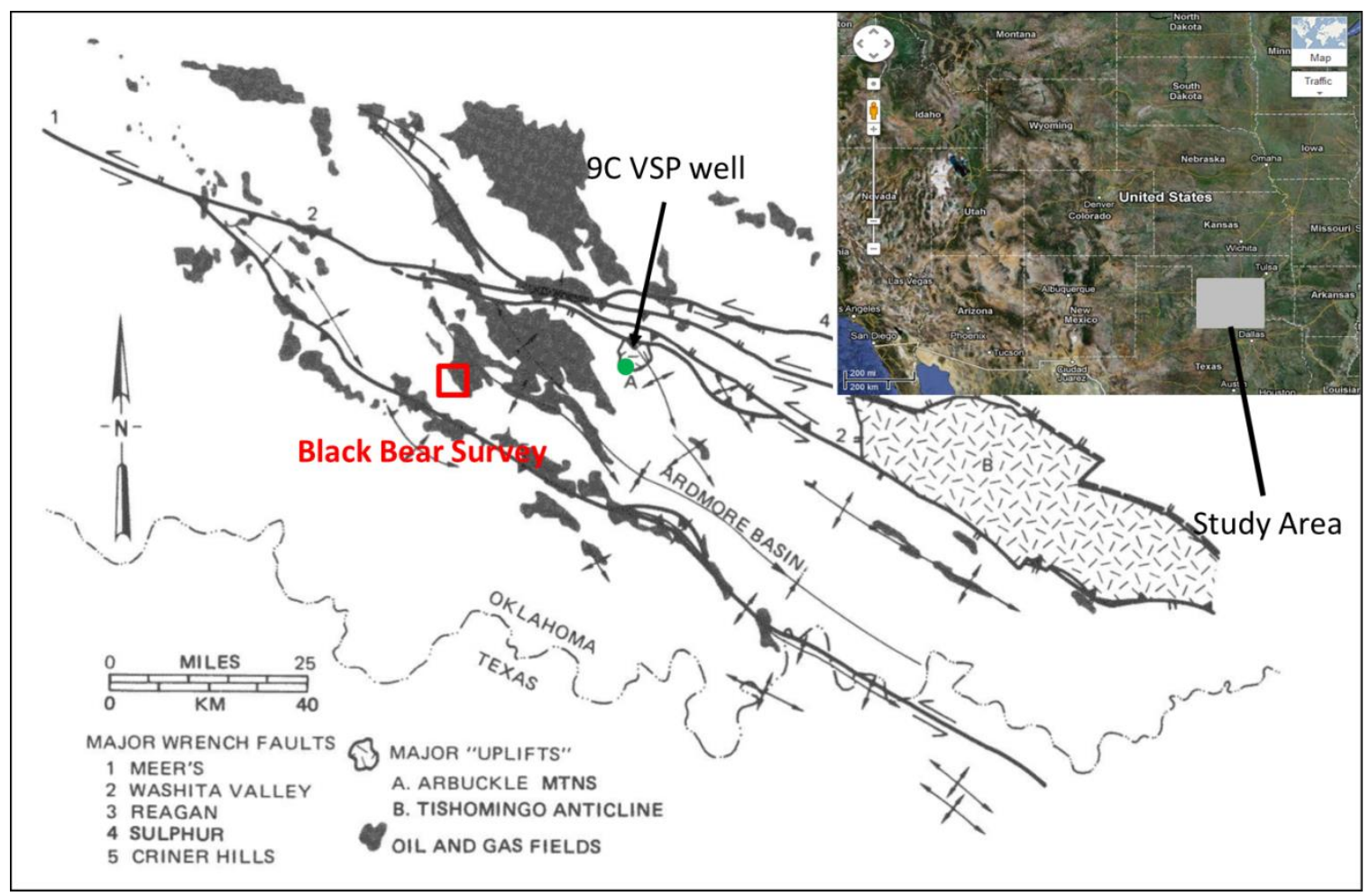

Figure 34: Late Paleozoic structures of the Washita Valley wrench fault system and tectonic setting of the Ardmore basin, Oklahoma also shown is the location of the 16 sq. miles seismic survey and the location of the outer well (Brady1 well) (modified from, Harding, 1983). 


\section{StRATIGRAPHY OF THE ARDMORE BASIN}

Figure 34 summarizes the tectonic framework as previously discussed which creates the basinal structures for prograding sediments. Early stratigraphic evidence for tectonic activity in the Ardmore Basin area occurs from Lower Paleozoic fault blocks, which consists of sediments of Cambro-Ordovician through Early Devonian age and is associated with basement-rooted fault blocks. Lithologies include dolostone, subordinate limestone and sandstone. The productive sediments have three subregions; the Arbuckle Carbonate, the Simpson sandstone and the Hunton Carbonate. A complete stratigraphic section is given in Figure 35.

The Silurian-Devonian section consists of shallow marine carbonates that form the Hunton section and showed small production of oil and gas in east Oklahoma Anadarko Basin as early as 1921. Carbonates of the Hunton Group were deposited in a shallow marine setting, but the strata thicken into the deep Ardmore Basin as a result of higher syndepositional subsidence at the Wichita Fault Zone. An area of interest and the target of the Black Bear 3D-9C seismic survey is the Penn Sycamore Fractured Carbonate in the Southern Oklahoma folded belt. Sycamore reservoirs produce both associated and non-associated gas where the reservoir developed in Osagian rocks (Cole, 1988) associated with the complex faulted anticlines. The Sycamore formation unconformably overlies the Mississippian Woodford Shale and is conformably overlain by the Caney Shale which is not regionally deposited.

Above the Sycamore formation lies the Pennsylvanian Springer Formation consisting mainly of shale and sandstone. It accumulated in a northwest-southeasttrending marine basin, roughly parallel to the Southern Oklahoma aulacogen. The sediments were deposited on deltas to the northwest; near Ardmore and Lake Murray, the dominant environment of deposition is moderately deep-marine and the sandstones 
appear to represent a series of marine bars. The Springer formation is among the most prolific gas producing reservoirs in the basin.

Because of the restricted seas during the late Mississippian and Early Pennsylvanian (Frezon and Jordan, 1979) and subsequent erosion around the edges of the basin, gas sands of the Springer formation are limited to the deep, lower-shelf regions of the Anadarko Basin. There is a sequence unconformity at the top of the Mississippian (top of the Mississippian unconformity) that can be seen on the seismic section and is easily identifiable. The Brady Ranch 1 well with a 9C VSP survey well (Figure 36) also shows the top of the Mississippian unconformity seen in the change in the orientation S1S2 shear polarization (fracture direction) which is quite pronounced. The prospective Pennsylvanian production comprises of reservoirs whose sediments were deposited during the period of Pennsylvanian tectonics, which altered the dominantly shelf carbonate depositional setting of the Cambrian through Mississippian periods to one of relatively more siliclastic sedimentation throughout the midcontinent. Clastics were ultimately derived from erosion of prominent exposed Pennsylvanian highlands. Transgression of the early Morrowan Sea toward the north and northwest over the prePennsylvanian unconformity and subsequent regression resulted in deposition of sand bodies that now form reservoirs.

Most of the Permian sediments consist primarily of dolomitized carbonate bank deposits in the Chase Group, porous limestone reservoirs being abundant in the upper formations of the Council Grove Group and discontinuous fluvial sandstone bodies of the Sumner and Oscar Groups. The shallow reservoir interpreted on the seismic section is equivalent to the unconformity that overlies the Hennesy Group, which is represented by a strong bright reflector. 


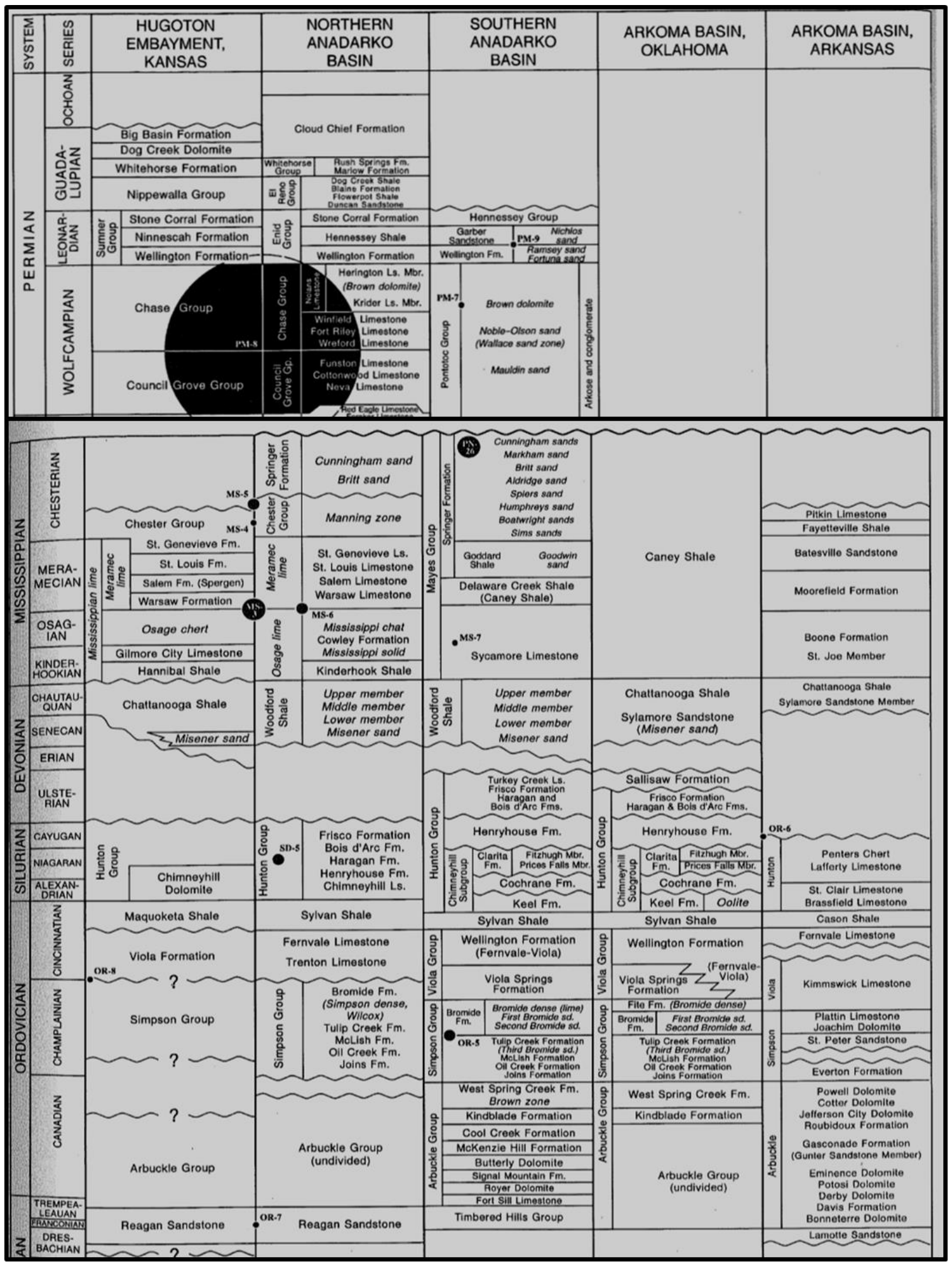


Figure 35: Generalized surface and subsurface stratigraphic columns for major sedimentary basins in Oklahoma. Formal stratigraphic names in standard print informal unit names in italics. Formal formation names are not necessarily differentiated. Local informal nomenclature from Pennsylvanian units varies in the southern Anadarko Basin. Double columns of informal names indicate different local usage and unknown correlation of units. The area of black circles represents relative cumulative gas production. Compiled primarily from Cipriani (1963), Hills and Kottlowski (1983), Mankin (1986). Other sources are Amsden (1979, 1980, 1989), Amsden and Sweet (1983), Johnson (1988), Hester and others (1990), Baars and others (1991), Carpenter and Evans (1991), Davis and Northcutt (1991), Wilson and others (1991), Knode and others (1993). 


\section{9-C VSP ACQUISITION IN THE TEXACO BRADY RANCH -1 WELL}

A multicomponent (9C) VSP survey in the Texaco Brady Ranch 1-5 well was acquired in Carter County, Oklahoma in November 1992. Two horizontal shear vibrator sources were employed to record 63 depth levels, with sweeps running from 5 to $50 \mathrm{~Hz}$. In the deep section, 2440 to $3010 \mathrm{~m}$ (8000 to $9875 \mathrm{ft})$, data were recorded at $23 \mathrm{~m}(75 \mathrm{ft})$ intervals (Beckham, 1996). The well was nearly vertical, and the sources were close enough to the wellhead so that corrections for non-vertical ray paths were negligible, allowing computational Alford rotation analysis for estimates of anisotropy. Historically this has been a necessary condition for synchronous rotation processing (Alford, 1986; Peron, 1990) applied in the polarization shear-wave analysis for fracture characterization. The downhole recording tool deployed in this uncased well consisted of a threecomponent geophone module combined with a magnetic inclinometer, which determines the orientation of the module with respect to magnetic north with an accuracy of $\pm 2^{\circ}$. The inclinometer consists of a three-component accelerometer and magnetometer to estimate the local magnetic field, and determines the tool orientation with respect to magnetic north. The VSP survey was conducted in the open hole, prior to casing and cementation.

The polarization orientation of the fast (split) shear component versus depth curves are shown in Figure 36 (a). The two curves represent the result from conventional synchronous matrix rotation (Alford rotation analysis) at each depth level and the result of applying a downward-continuation process of the two recorded horizontal shear components (Ohanian and Beckham, 1992). The data resulting from the conventional rotation analysis of zero-offset data that are quite erratic below the Mississippian unconformity (1980 m, $6495 \mathrm{ft}$ ). Note many depth levels are absent on the curve because two separate shear components could not be ascertained after rotation. Both curves show a strong change in orientation just below the Pennsylvanian-Mississippian unconformity 
at $1920 \mathrm{~m}(6300 \mathrm{ft})$. The orientation from the downward-continuation processing is $\mathrm{N} 60^{\circ}-75^{\circ} \mathrm{E}$ in the carbonate zone below the Top of Mississippian unconformity $2622-$ $2990 \mathrm{~m}$ (8600- $9810 \mathrm{ft})$. This observation provides evidence of a significant change in fracture orientation above and below the top of the Mississippian Unconformity.

Estimating polarization direction by synchronous rotation depends on having identical off-diagonal (xy and yx) shear-wave components at common arrival times (Winterstein and Meadows, 1991b). This condition can be violated if S-wave polarization changes with depth caused by changes in vertical crack or fracture orientation. Downward continuation algorithms (Ohanian and Beckham, 1992) have been developed to process VSP data in this case. The methods remove the lag time between fast and slow split shear waves in the overburden and simulate placing the sources at depths where polarization changes occur. Analysis of the cross-dipole data for fracture orientation can be performed by synchronous rotation of the data matrix if it is assumed that any set of vertical fractures that is encountered maintains a constant orientation. From the shallow data (above about $6000 \mathrm{ft}$ or $1800 \mathrm{~m}$ ) in Figure 36.A, we see that the $\mathrm{S} 1$ orientation is essentially N-S. The polarization analysis of the surface seismic data is entirely within this interval.

Figure 36 (b), shows the normalized time delay between the split shear components versus depth at each level. The relatively small change in delay in the interval 1070-1829 m (3510-6000 ft) may indicate a nearly isotropic section above 300m. A significant change in observed delay times coincides with the top of the Mississippian unconformity, within an approximately $90^{\circ}$ orientation change from north-south to $\mathrm{N} 80^{\circ}$ $85^{\circ} \mathrm{E}$ above the top of Mississippian unconformity. A change in polarization (birefringence) can be expected if the orientation changes on the order of $90^{\circ}$, with a subsequent reversal in the leading split shear component (Martin and Davis, 1987). 
Another significant reversal in delay times occurs at the $2530 \mathrm{~m}(8300 \mathrm{ft})$ level within the Caney shale and corresponds to the abrupt orientation change in this interval. The possibility of a thin layer at $2500-2560 \mathrm{~m}(8200-8400 \mathrm{ft})$ with a different orientation in the third anisotropic layer (1982-2988 m, 6500-9800 ft) is indicated in Figure 36 (b). This depth corresponds to the Caney shale unit above the carbonate units. The Sycamore formation, which is a primary target for the Black Bear seismic survey depth ranges between $8600 \mathrm{ft}(2540 \mathrm{~m})$ to $10000 \mathrm{ft}(3050 \mathrm{~m})$ with a fracture orientation of $70^{\circ}$ from north. Note the Sycamore is a highly fracture limestone and the time delay in S1 and S2 shows the highly fracture nature of the limestone.

Further, we also saw evidence of changes in the S1, S2 delay times, a proxy for fracture intensity. The focus of my analysis to correct for polarization distortion from a single source polarization applied to a reflection on the better quality surface seismic represented at the much shallower age Hennessey Group unconformity. The Texaco Brady-1 well shows the upper section interpreted in the seismic section has a consistent fracture orientation and a delay in $\mathrm{S} 1-\mathrm{S} 2$ travel times. We will consider this orientation both before and after polarization correction. The surface data I use for the present analysis is at least $30 \mathrm{~km}$ from this subsurface information but there may be some consistency of the subsurface information.

In addition to the direct shear VSP data, examination of the cross-dipole log (Beckham 1996) responds to possible fractures and microcracks that are smaller in length or diameter than the large seismic wavelength, with sonic wavelengths of $0.9-0.3 \mathrm{~m}$. Three distinct azimuthal anisotropy orientations were obtained from processing a limited amount of cross-dipole shear-wave log data. Two of the orientation sets (a northeast set and a east-southeast set) correspond to orientations measured in the core (Figure 37). The northeast set corresponds to orientations obtained from the VSP in the interval 2710-2896 
m (8889-9499 ft). Thus, the VSP may be responding to a combination of macrofractures and microcracks that are aligned subparallel in a northeast direction. Another nearly north-south orientation in the cross-dipole data has not been confirmed in the other data sets. The north-south and east-southeast orientations from the crossdipole data may indicate the presence of a system of microcracks of generally random orientation in the carbonate interval, which have little influence on the VSP, but show up on the crossdipole data as distinct orientations in certain thin intervals. 


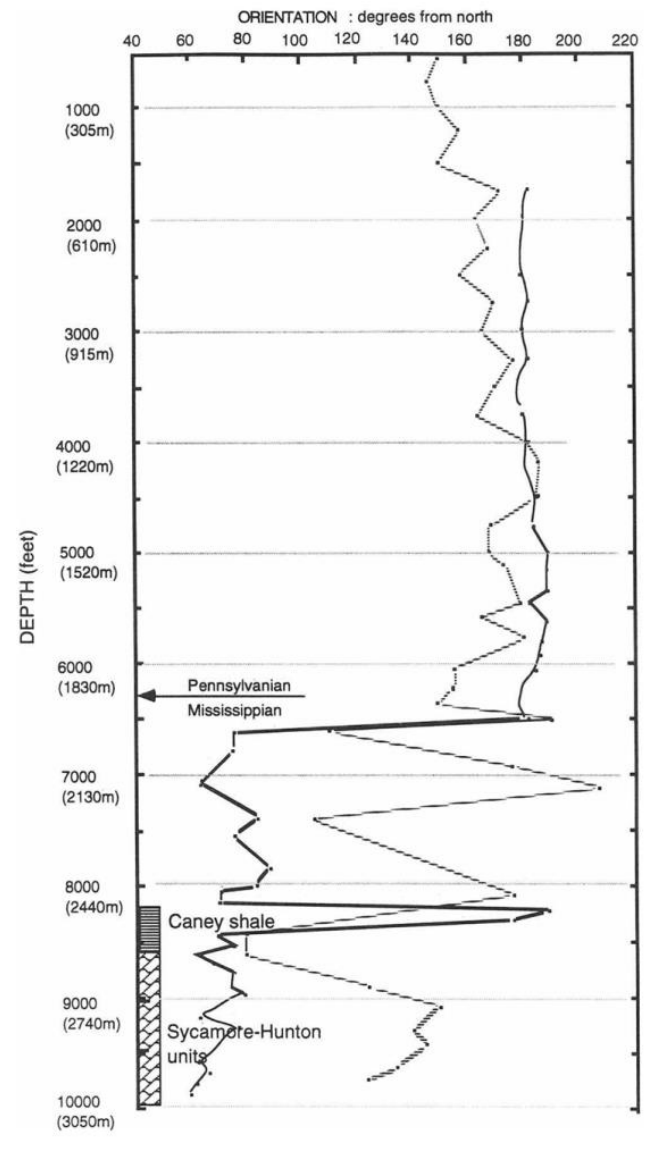

Figure 36.A: Orientation of azimuthal anisotropy curves versus depth from analysis of Brady Ranch 1 VSP data. The dashed curve is a result of using only Synchronous Rotation(SR) independently at each depth level. The solid curve is the result of applying the downward continuation algorithm plus SR. This curve is useful to estimate orientation of vertical fractures at a given depth. Note the abrupt change at the top of the Mississippian unconformity (Beckham, 1996).

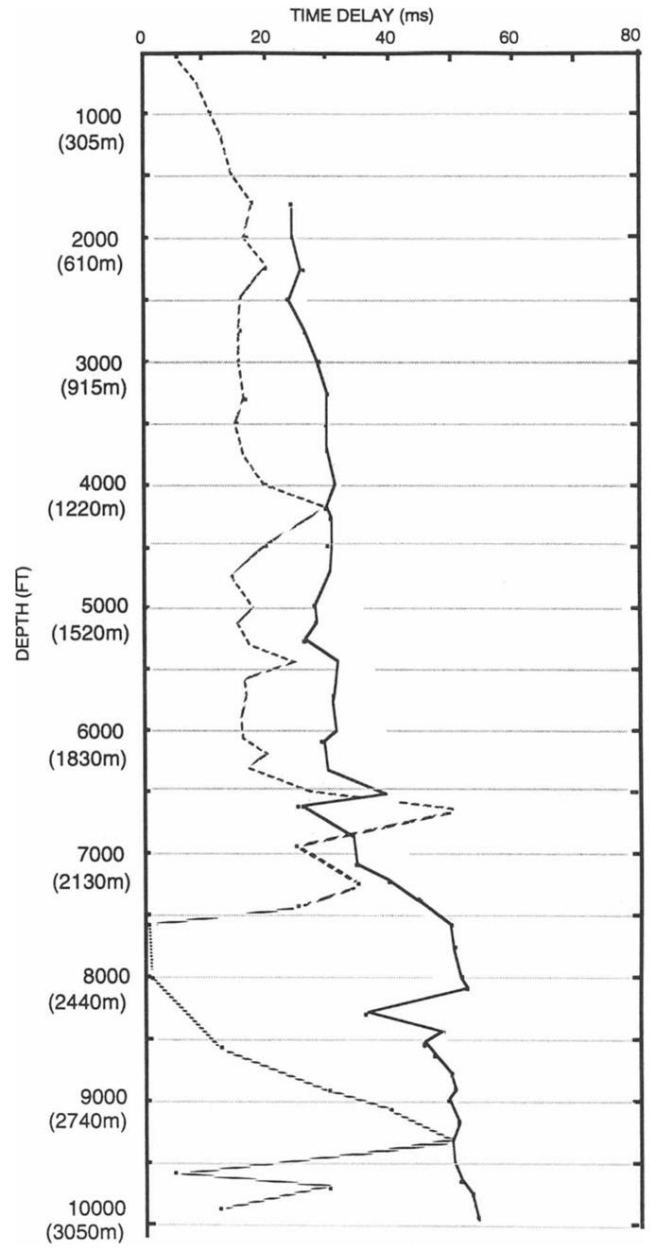

Figure 36.B: Time delay between fast and slow split shear waves from analysis of the Brady Ranch 1-5 VSP data. The dashed curve is the result of applying Synchronous Rotation at each depth level, and the solid curve is the result of applying downward continuation plus SR. The time delay is often used as a proxy for fracture intensity (Beckham, 1996). 


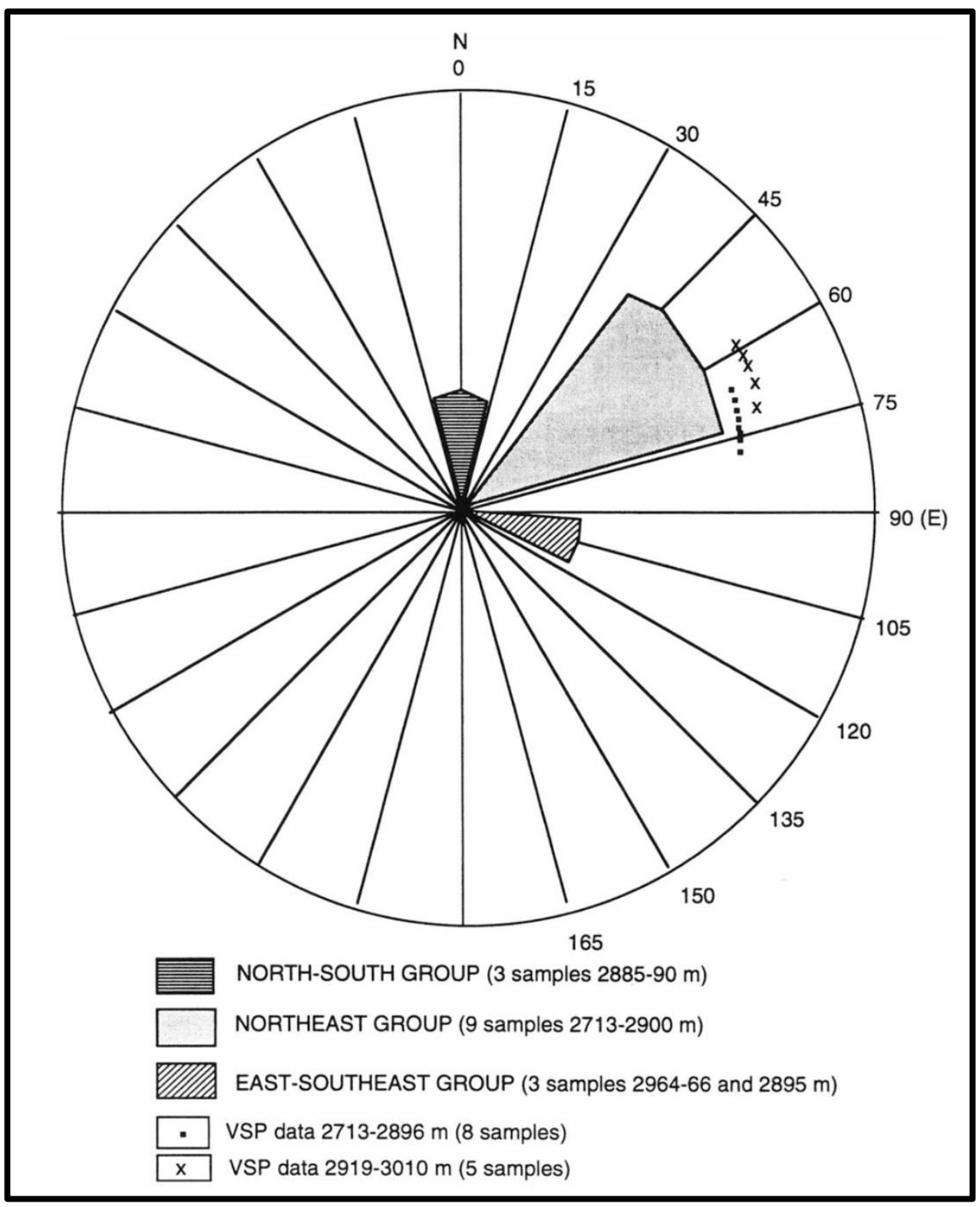

Figure 37. Diagram showing orientation of azimuth anisotropy as obtained from crossdipole log data analysis in specific depth intervals below $2700 \mathrm{~m}(9,000 \mathrm{ft})$ in the carbonate section. Orientations from the VSP data are also indicated (Beckham, 1996). 


\section{C-3D SEISMIC SURVEY}

The Black Bear Creek data is a 3D 9C data set that was acquired in 1998 by the PGS land team and given to the Exploration Geophysics Laboratory (Bureau of Economic Geology, The University of Texas at Austin) in Stephens County, Oklahoma. The main geologic objective of this survey was to image the Sycamore formation (a fractured carbonate) and estimate fracture parameters. The seismic survey covers 16.2 square miles (Figure 38). The Pennsylvanian Sycamore Carbonate was expected to be fractured at depth as documented in 9-C VSP and borehole dipole log data survey in the Texaco Brady borehole located in the Carter County (Beckham, 1996) 18.75 miles ESE of the surface seismic survey. The VSP and cross-dipole log acquired was discussed earlier in this chapter. First we address the surface seismic data and how we observe the polarization distortion and applied the correction to a single source polarization. Figure 39 shows the surface acquisition configuration of the seismic survey with 2 component shear sources (Vibroseis), explosive P-waves and 3 component receiver locations. Note the regular pattern of the survey. The data were gathered by progressive source traverses from west to east with receivers deployed in north-south lines, which resulted in a wide array of source-receiver azimuths and offsets--providing a range of reflection incidence angles. 


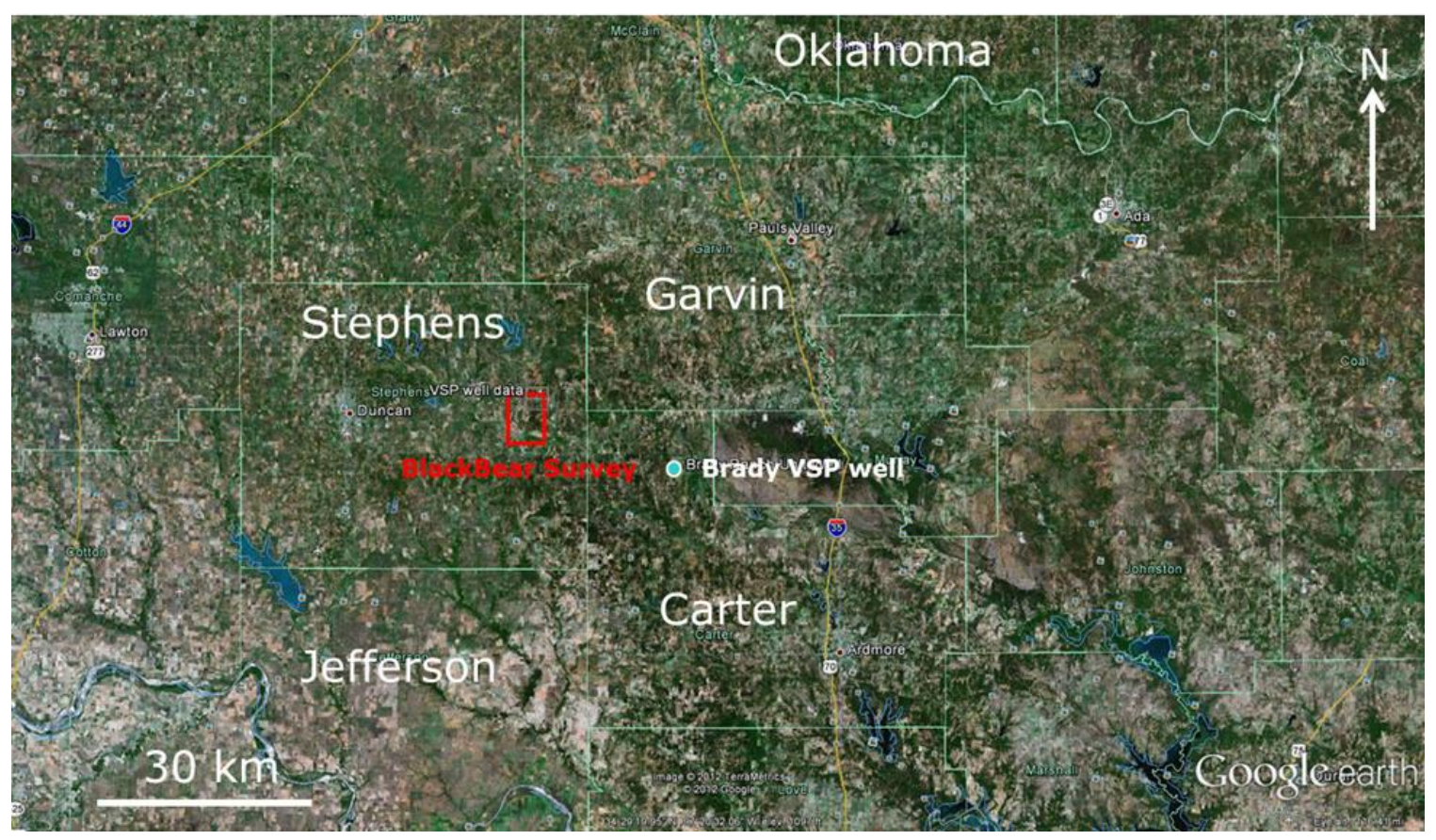

Figure 38: Google Earth image showing the surface Ardmore Basin in Oklahoma with the different counties and fluvial distributaries. The red box outlines the area of the BlackBear survey and the blue circle is the Texaco Brady Ranch 1-5 well multicomponent VSP and cross-dipole shear wave log. 


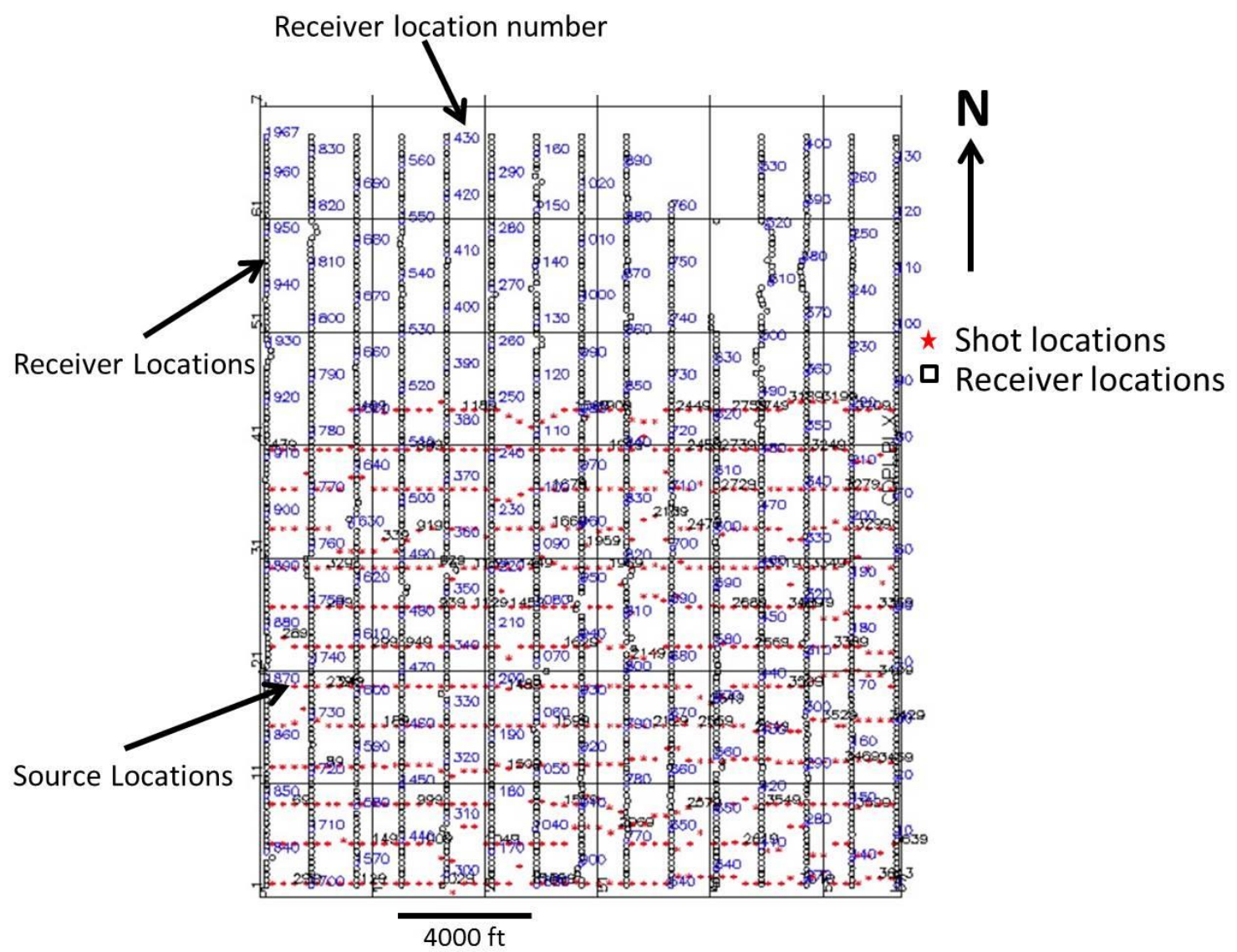

Figure 39: Diagram of Source and 3-C Receiver pairs. The direct primary wave data were acquired using an explosive source but the S-wave wave data was acquired using Vibroseis sources. Total surface area covered is about 16 square miles. Source lines are about 350 meters apart and receiver lines are 420 meters apart. Note the concentration of seismic source locations to the southern portion of the survey, limiting the concentration of high quality reflection data to that area. 


\section{Seismic Acquisition Parameters for 3D-9C Survey}

The survey geometry and subsurface binning was designed to provide high common midpoint (CMP) fold (360) at the center of the survey and continuous distribution of seismic-receiver offsets and azimuths in each CMP bin over the area of the survey. The seismic P-wave sources consisted of explosive charges in shot holes for the P-wave data and separate $\mathrm{x}, \mathrm{y}$ horizontal vibrator sweeps used for the shear-wave data. Thus, two sets of orthogonal shear-wave polarization data, as well as P-wave data were recorded at each source location. Further, mode converted P-SV data from the explosive sources were observed on the horizontal components. Each receiver location consisted of an array of 3 component geophones. Recording the seismic source signature was important to balance the source response of different source directions. The acquisition was implemented as a moving sequence of source position into a fixed pattern with all receiver stations in a "notch" live and recording during each source (x,y and $\mathrm{z}$ ) excitation. The survey was composed of $15 \mathrm{~N}-\mathrm{S}$ receiver lines spaced at 420 meters and $13 \mathrm{E}-\mathrm{W}$ source lines spaced at 350 meters apart. Each source point was recorded three times, 2 components of Vibroseis sources for S-waves and single shot P-wave data shot hole sources. There were a total of 2220 receivers and 1197 shots. Table 4 summarizes

details of the acquisition parameter of the 3D-9C seismic survey with the associated record lengths and receiver array. 


\begin{tabular}{|l|l|}
\hline P-wave source record length & 4 seconds \\
\hline Shear wave source record length & 6 seconds (after de-sweep) \\
\hline Primary wave source sampling interval & $4 \mathrm{~ms}$ \\
\hline Shear wave source sampling interval & $8 \mathrm{~ms}$ \\
\hline S-wave source Vibroseis sweep & $\begin{array}{l}6-48 \mathrm{~Hz} \text {, eight, 16 sec sweeps } \\
\text { per "shot" }\end{array}$ \\
\hline Receiver station lines & 15 \\
\hline Total receiver locations & 672 \\
\hline Live receiver stations for each source excitation & 2220 \\
\hline Receiver station spacing & $165 \mathrm{ft}$. \\
\hline Receiver station line spacing & $1320 \mathrm{ft}$. \\
\hline Total source lines & 13 \\
\hline Total source locations & 1197 \\
\hline Source spacing in the source line & $330 \mathrm{ft.}$ \\
\hline Shot line spacing in the receiver line & $1155 \mathrm{ft}$. \\
\hline Maximum recording channels & 2220 \\
\hline Areal extent of survey & $16.42 \mathrm{sq.} \mathrm{miles}$ \\
\hline Total traces recorded & 602280 \\
\hline Total CMP bins in survey & 33630 \\
\hline Bin width (East - West) & $165 \mathrm{ft.}$ \\
\hline Bin height (North-South) & $82.50 \mathrm{ft.}$ \\
\hline Maximum CMP fold & 360 \\
\hline Maximum source receiver offset & $9912 \mathrm{ft.}$ \\
\hline
\end{tabular}

Table 4: Table shows some of the survey statistics of the Sycamore Oklahoma Prospect 


\section{CMP Fold, SOURCE-RECEIVER OFFSET AND SOURCE-RECEIVER AZIMUTH:}

In many onshore exploration areas, the land surface is covered with a relatively thin layer of material of low seismic velocity. Commonly known to be the weathered layer, it is generally related to aerated material above the water table or to geologically recent unconsolidated sediments, such as alluviam or glacial on a substratum of harder consolidated rocks. Variation in the physical properties of this upper layer can cause a dramatic deterioration in the quality of land seismic data. Before we can properly process and analyze seismic data, we need to correct for this layer, commonly referred to as static corrections.

The denser distribution of source positions in the southern portion of the survey area allowed a high CMP fold in the center of the survey. The CMP fold distribution has a concentric pattern within the survey area, reaching a maximum of 360 traces per bin in the center of the survey area (Figure 40).The full range of source and receiver patterns allowed a good distribution of source-receiver offsets, especially in the source-receiver offset range of $2000-8000$ feet. Except for the edges of the survey, there is an adequate distribution of offsets, ranging from 1,520 to 10640 feet (Figure 41). The full range design of source-receiver locations assures a good distribution of individual sourcereceiver azimuths distribution in a bin at the center of the survey. Figure 42 shows the azimuth distribution of the seismic data where the color represents the number of traces that have the individual source-receiver offset and azimuth ranges respectively. A broad range in the source-receiver offset and range in source-receiver azimuth is also essential to the analysis of reflection amplitudes with offset for lithology and fluid content estimation. A range of source-receiver angles provides the information for analysis of the effect on polarization distortion and how the correction is applied. 


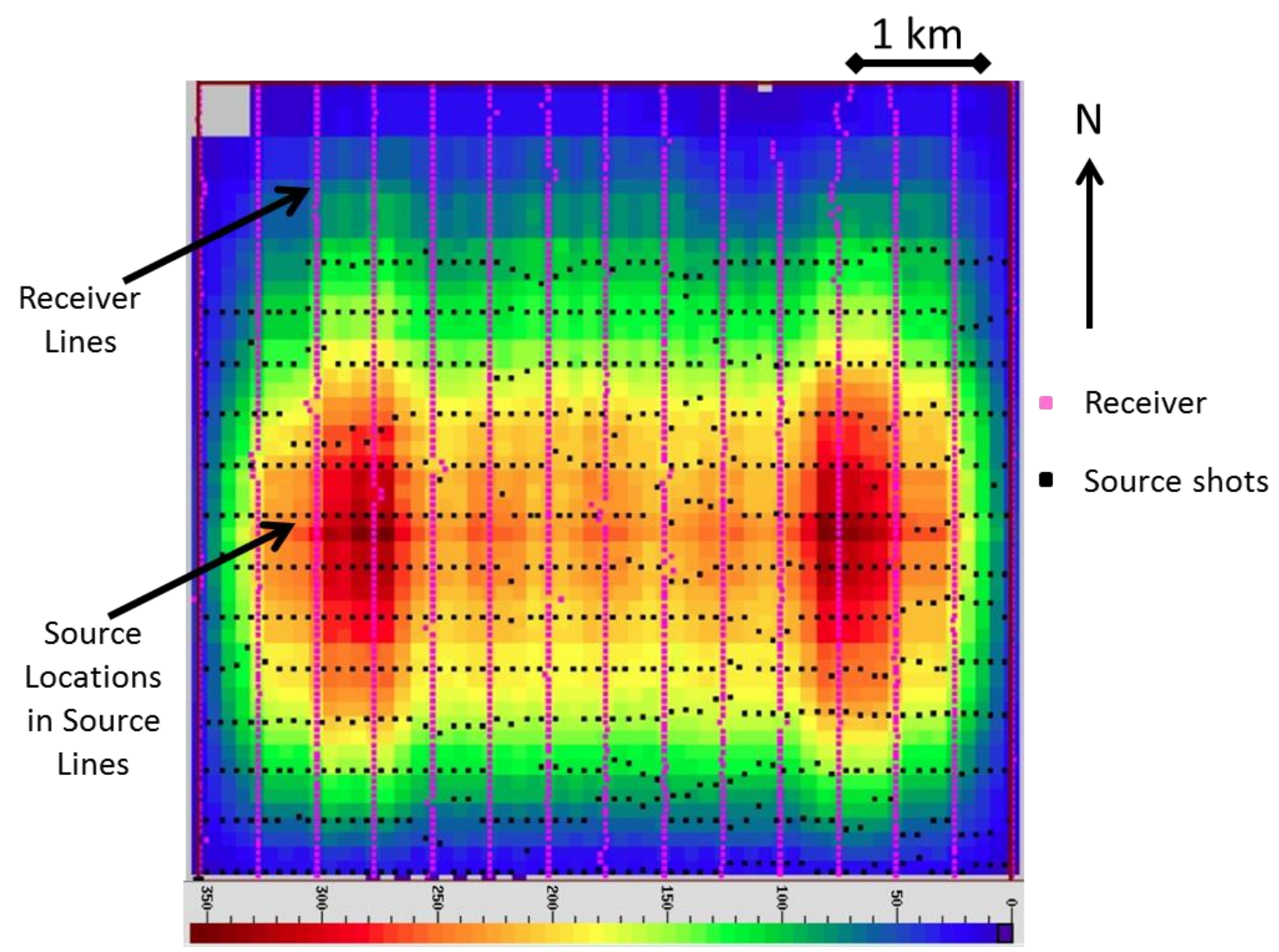

Figure 40: A plot of CMP fold showing 360-fold coverage over the center of the survey for an X Source - X Receiver orientations. The edge of the survey has lower fold because several of the first and last shots do not reach as many receivers as in the central part of the survey. 


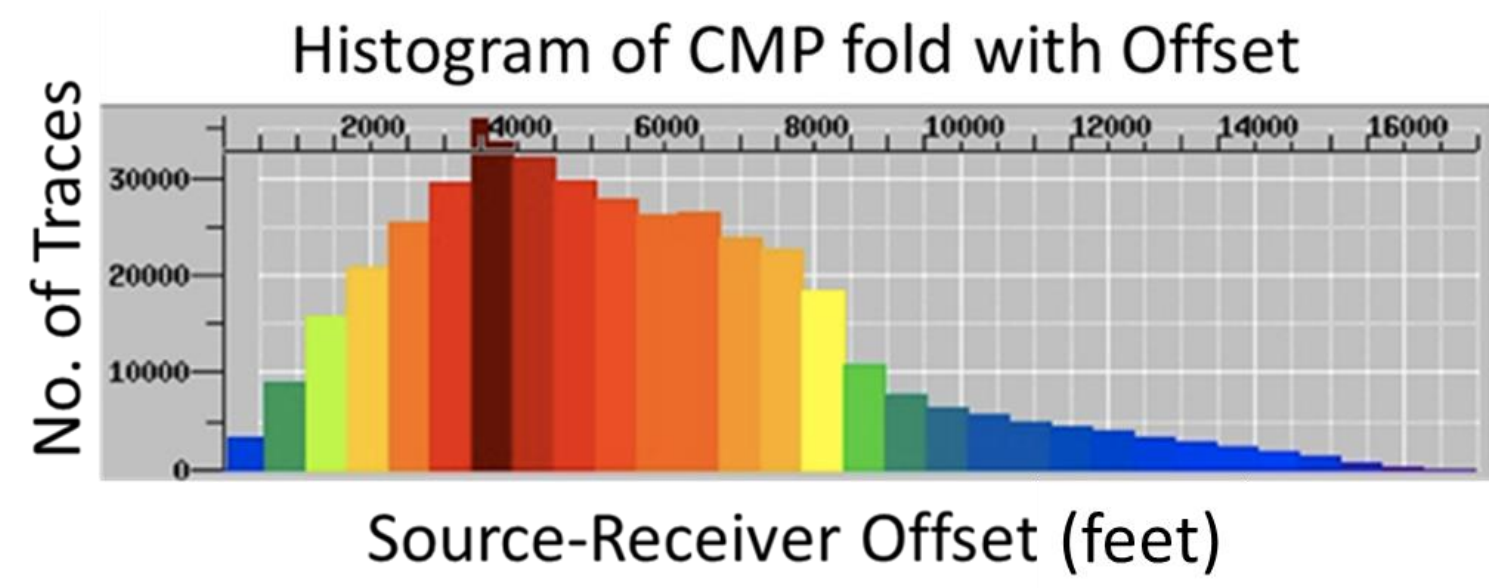

Figure 41: Histogram showing the source-receiver offset distribution over the entire survey. Note the different offset distribution between 2000-9000 ft.

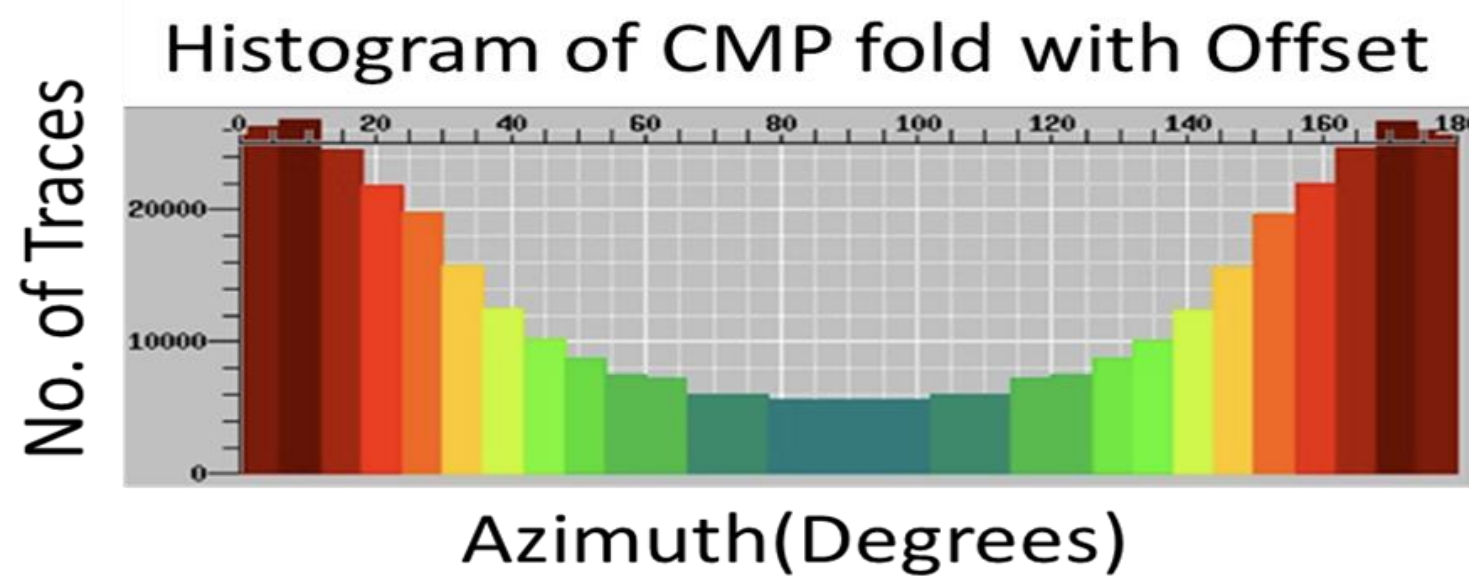

Figure 42: An azimuth distribution histogram showing the number of traces over the entire survey not for each CMP with the associated its azimuths. 


\section{Processing of 3D-9C Reflection Data}

As in most land surveys, perhaps the most important steps for preliminary processing the data of Black Bear survey data were those related to near-surface static corrections, coherent noise and amplitude recovery and compensation. Fortunately, multiple reflections, which are often a major problem for many surveys, do not present a problem in this survey. The efforts to enhance seismic signal quality were directed toward near-surface effects, pre-stack noise attenuation and enhancement of higher frequencies with broader overall bandwidth and improved imaging from such signal bandwidth improvement. After preliminary seismic processing which included defining field geometry, PSG applied signal enhancement, CMP sorting, velocity analysis and normal move-out of shear-wave components correction to the Black-Bear shear-wave data survey. Each source component was ready for analysis and rotation into their natural polarization direction. This was done in order to avoid distortion associated with anisotropy, so that only pure components of either S1 or S2 are being studied and processed. The general processing flow for the shear wave data is listed in Table 5.

One of the main considerations in processing the Blackbear 3D data set is the preservations of the original reflection amplitude which is applied to the polarization

distortion correction. These data were processed with a surface consistent method of shot and receiver locations for correcting the amplitude unbalance among different shot and receiver pairs. This unbalance may be caused by variations in the source energy from shot to shot, ground coupling of sources or receiver, variations in geophone array response and variations in the ground compensation.

Two other processes also contribute to amplitude correction: Q compensation and deconvolution where $\mathrm{Q}$ compensation corrects for attenuation, resulting in a more stable 
wavelet. Deconvolution was applied to optimize the Fourier amplitude spectrum, especially at higher frequencies.

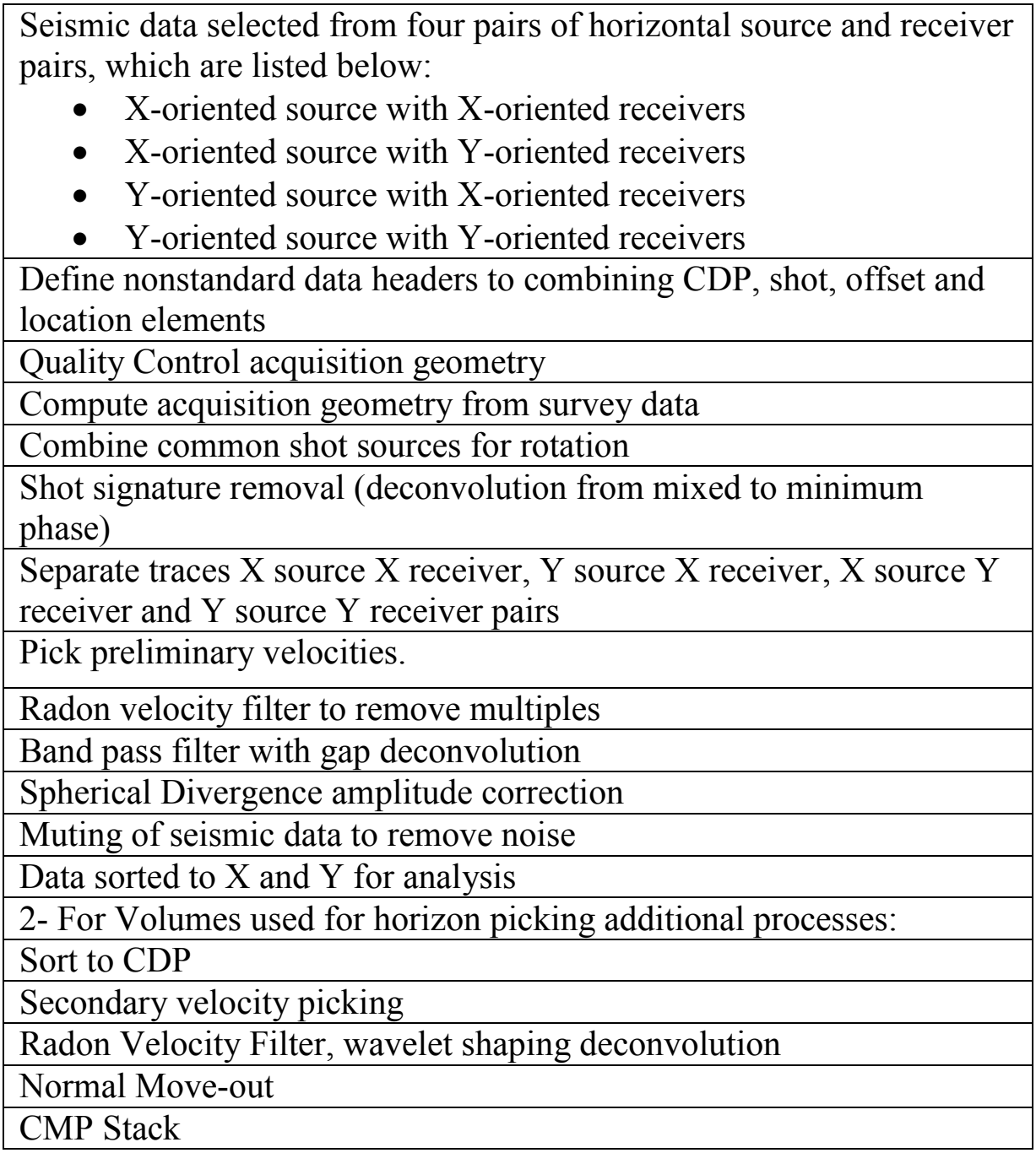

Table 5: Direct shear-wave processing flow

Time varying processes such as Amplitude Gain Correction (AGC) are not recommended for the polarization distortion correction, because they distort the relative amplitudes within the gather. Such corrections were not included in the amplitude 
preserving sequence used here and special attention was dedicated to noise attenuation and static corrections. This processing led to the $\mathrm{P}, \mathrm{SV}$ and $\mathrm{SH}$ reflection volumes used for the preliminary interpretation in Figures 44 and 45

\section{Post-STack Data}

The fully processed P-wave seismic data set was initially interpreted to determine the structure and stratigraphy. Due to overall data quality and resolution issues, the Pwave data tend to be the best starting point for structural interpretation, I was able to identify shallow reflecting horizons for the application of the polarization distortion correction which is the subject of this Dissertation. Figure 43 shows the structural and stratigraphic features, features such as the top of the Mississippian unconformity, are identified by their high amplitude character. Below the unconformity the seismic data is somewhat chaotic in character. The Sycamore carbonate, the principal target for the

original seismic survey is difficult to identify due to poorer imaging quality, below about one sec two-way reflection time (Figure 43). I will focus my analysis on a continuous reflection event that is well imaged above the Mississippian Unconformity.

The fully processed and stacked SH-wave data were carefully examined to help guide the interpretation of the pre-stacked seismic data set in order to provide guidance in the analysis of the polarization correction. Figure 44 shows the $\mathrm{SH}$-wave structure of the top of the Mississippian unconformity, which is identified by its high amplitude character. Below the unconformity the limited structure seen on the P-wave data set is not well defined on the S-wave data. The Sycamore carbonate, the principal target for the seismic data set is difficult to identify. We can also identify the chaotic seismic facies below the Mississippian Unconformity and planar continuous well imaged seismic data 
above the Mississippian Unconformity. There is good correlation between the $\mathrm{P}$ and $\mathrm{S}$ seismic data sets down to the top of the Mississippian Unconformity. The P and S wave data are displayed with an almost a 2:1 in the time scale ratio in order to compensate for differences in propagation velocity.

Note the structural similarity, particularly for the shallow Hennessey reflection between Figures 43 and Figures 44 . Figure 45 shows a side by side 3D view of the Pwave and S-wave profiles where the time differences between the two seismic volumes are adjusted to compensate for velocity differences. Note the difference in structural imaging between the two seismic volumes and the average image quality. We can also identify the top of the Mississippian unconformity on both seismic volumes as well as the shallow reflection event that will be used for polarization distortion corrections. Figure 46 and Figure 47 show the structural interpretation on both the $\mathrm{P}$ and $\mathrm{S}$ wave seismic data sets respectively of the Hennessey horizon that will be used for the polarization analysis evolution. Note that on the P-wave interpretation we have $80 \mathrm{~ms}$ of structural time difference between the shallowest and deepest part of the interpretation. Figure 47 we see "streaks" on the S-wave amplitudes, most likely associated with acquisition footprint associated with the surface distribution in the source and receiver positions. Seismic traces associated with these shallow reflecting horizons were then used to apply the polarization distortion correction to the pre-stack data. The deeper events were identified because they were use to correlate the time depth relationship between the P-wave and Swave data, but were not used in the polarization analysis. 


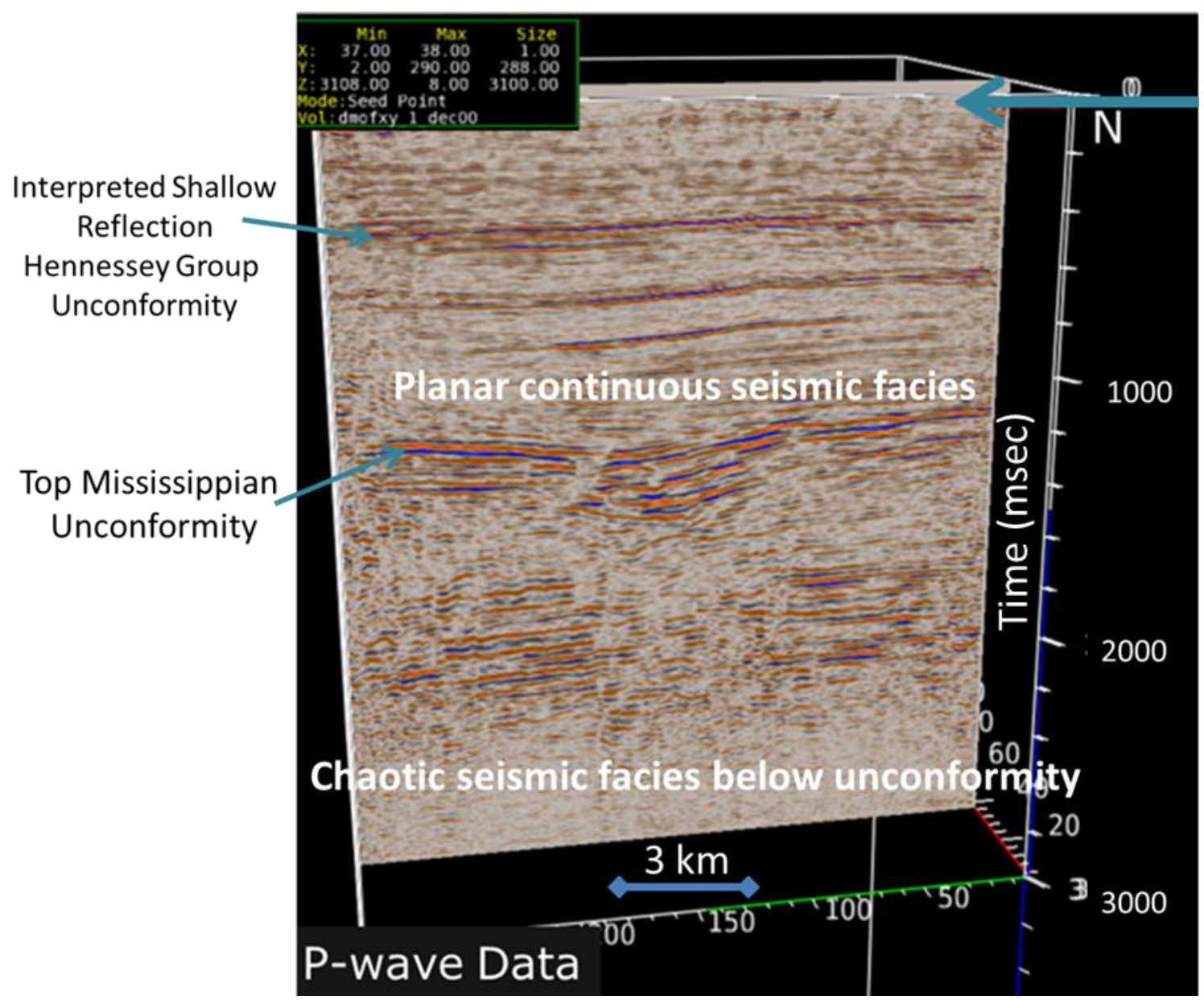

Figure 43: 3D stacked P-wave profile showing structure. Note the angular unconformity (Top Mississippian Unconformity) at approximately $1.25 \mathrm{sec}$ and the strong reflection range at $0.5 \mathrm{sec}$ which was used as the layer for polarization distortion correction. Note: Deterioration of data quality below the top of Mississippian and at Sycamore levels. Also note good quality shallow data above $1000 \mathrm{msec}$. 


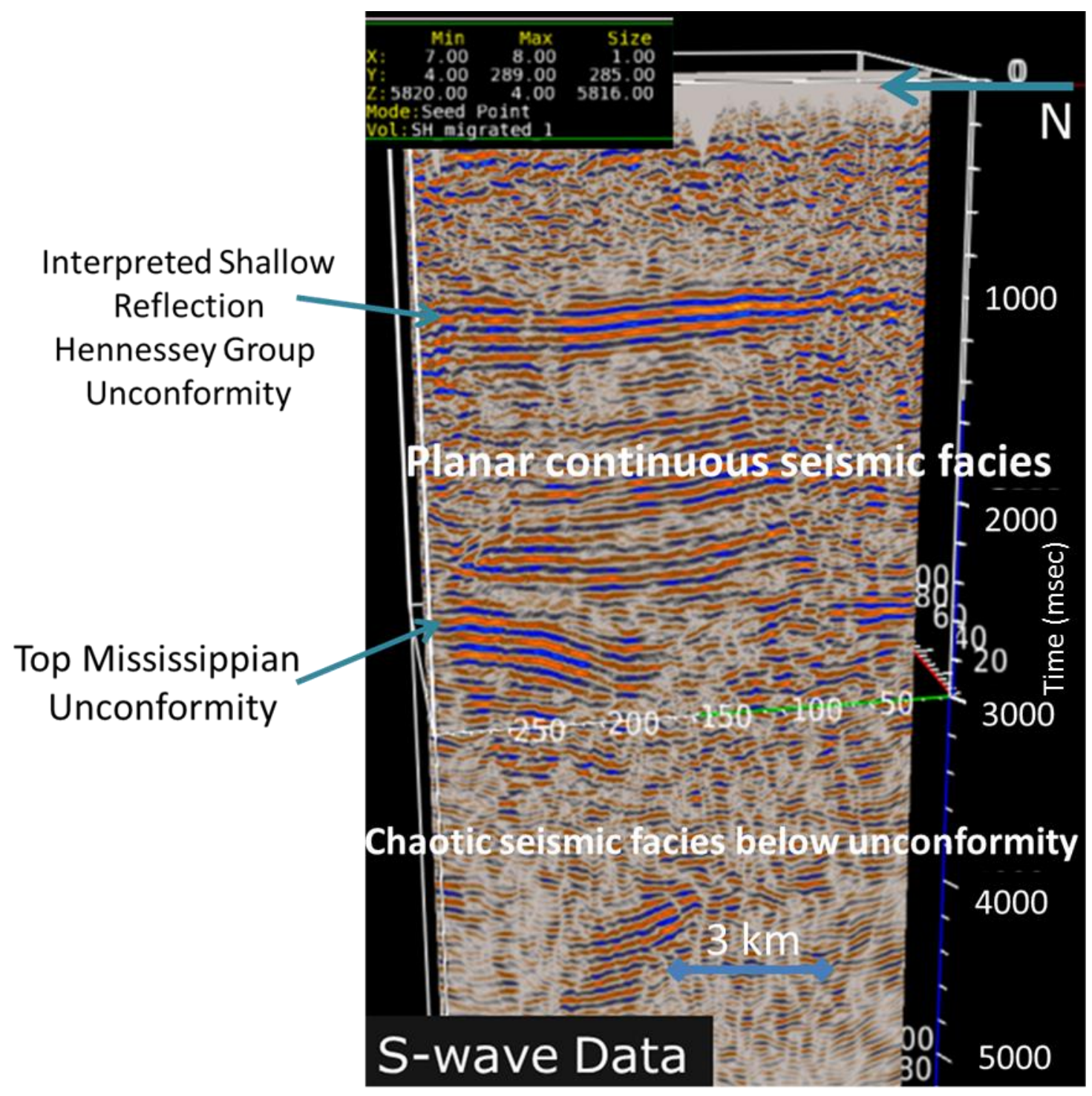

Figure 44: 3D stacked S-wave ( $\mathrm{SH}$ ) profile showing equivalent structure to the $\mathrm{P}$-wave data with lower seismic resolution in reflection time. When converted to depth the resolution with $\mathrm{P}$ is probably comparable. Note the unconformity at approximately $2.8 \mathrm{sec}$ and the strong reflection at $1.1 \mathrm{sec}$ which is equivalent to the P-wave surfaces that were identified. The deeper events are used in the registration not in the correction. Note: Deterioration of data quality below the top of Mississippian and at Sycamore levels. Also note good quality shallow data. 


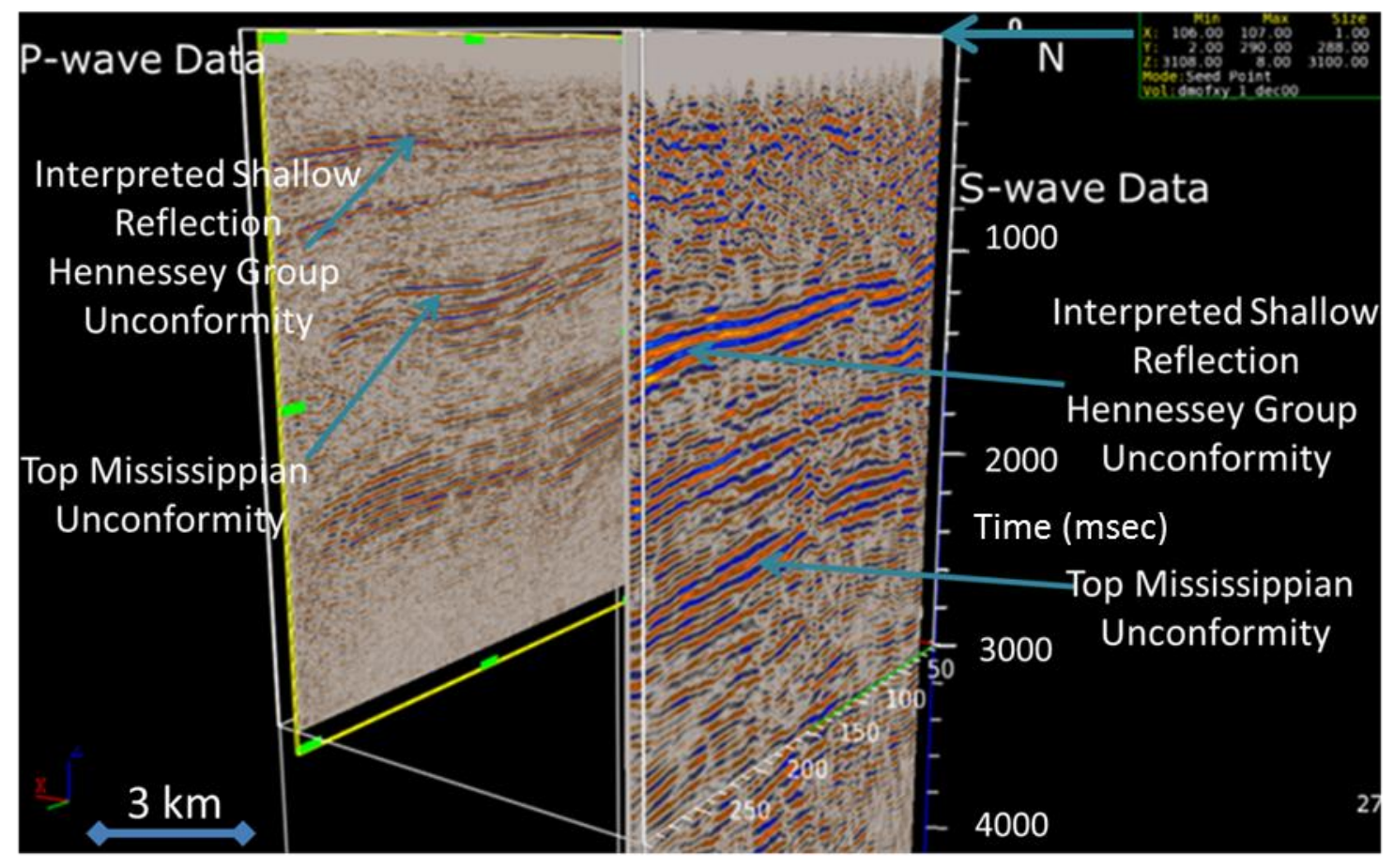

Figure 45: 3D image of profile showing both the $\mathrm{P}$ wave and $\mathrm{SH}$ wave profile where the resolution and structure can be quickly identified and reflections visually correlated between the $\mathrm{P}$ and $\mathrm{S}$-wave $(\mathrm{SH})$ data. Note the differences in reflection time between the two volumes, which are adjusted for difference in $\mathrm{P}$ and $\mathrm{S}$ propagation velocities and the structural complexity deeper in the section that is seen on the $\mathrm{P}$-wave volume is not seen on the $\mathrm{S}$-wave volume. Below the Mississippian unconformity on the P-wave seismic data we can still identify stratigraphy and structural complexity. Note below the Mississippian unconformity on the S-wave data we cannot see the same seismic resolution so interpretation is very difficult. 


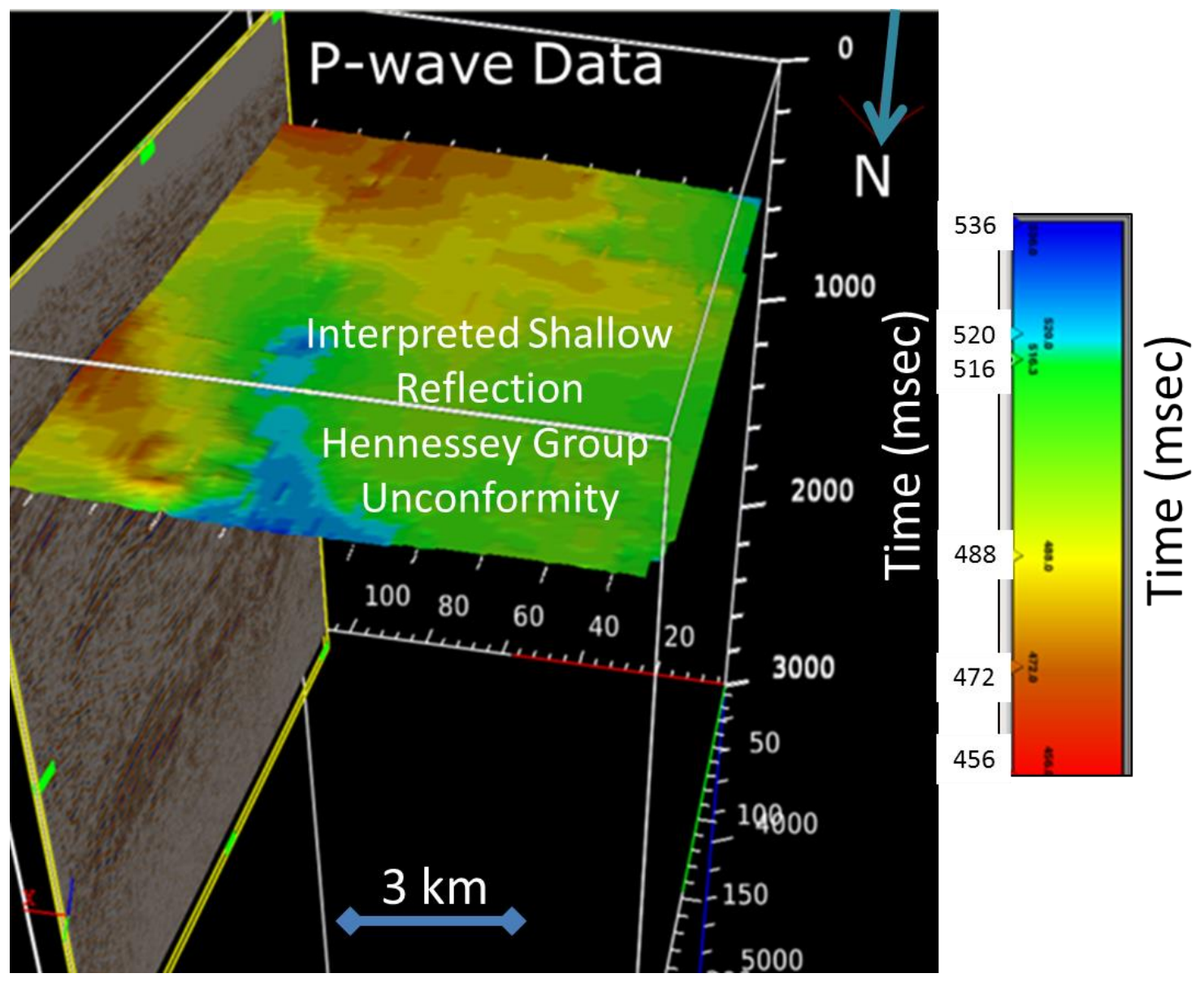

Figure 46: 3D display of $\mathrm{P}$-wave reflecting horizon showing little structural relief ranging from $456 \mathrm{msec}$ to $536 \mathrm{msec}$. The warm colors are shallower and the cool colors are deeper. The surface is dipping from North to South. 3D structure map of the shallow event on better image quality P-wave data. 


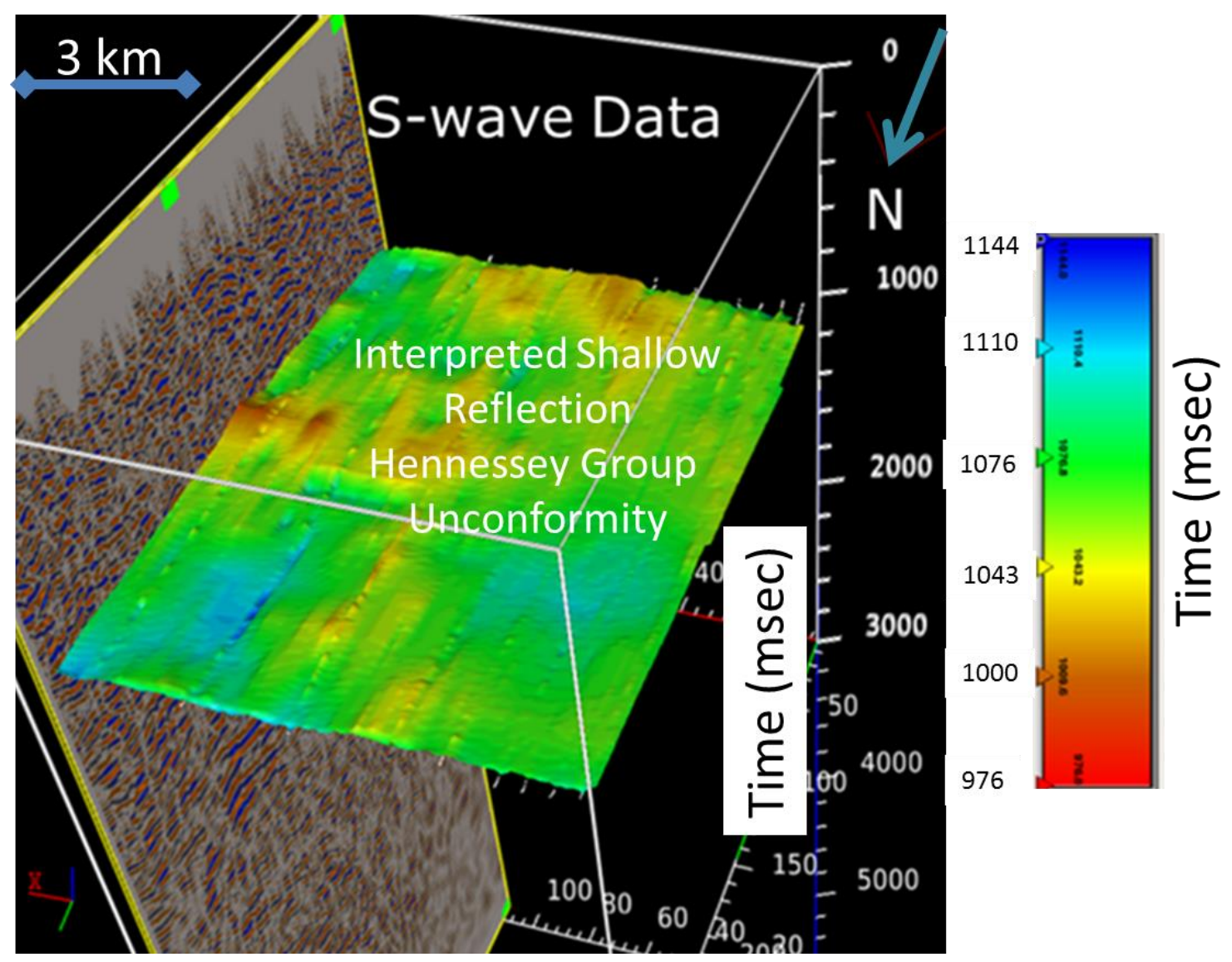

Figure 47: 3D time structure of S-wave (SH) horizon showing little structural relief ranging from $976 \mathrm{msec}$ to $1144 \mathrm{msec}$. The warm colors are shallow and the cool colors are deep. The surface is dipping from North to South. Note the "streaks" in the data associated with the acquisition footprint on the structure map which is very common in S-wave data acquisition and results from insufficient surface sampling at this relatively shallow depth. Note difference in reflection time for a common $\mathrm{P}$ and $\mathrm{S}$-wave $(\mathrm{SH})$ reflector, due to velocity differences. 


\section{POLARIZATION ANALYSIS OF THE PRE-STACK DATA}

After consideration of several stratigraphic levels (Hennessy group) on the poststack data volumes, a single reflecting horizon was selected for the interpretation and polarization analysis of the pre-stack shear-wave data. As discussed earlier, this analysis is focused on the better quality shear-wave data in the shallower portion (above the top Mississippian unconformity). The objective of this analysis is to experimentally confirm the existence of the polarization distortion in field observations provide a potential analysis of the distortion due to the reflection process and the ability to correct for such distortion. First, numerous receiver traces from a single source polarization (X) at a single source position were selected and organized into $\mathrm{X}$-oriented and $\mathrm{Y}$-oriented horizontal components recorded at each receiver position in a subset of the surface receiver position. I was then able to apply the polarization distortion correction to the combined $\mathrm{X}$ and $\mathrm{Y}$ receiver components of the recorded data. Figure 48 illustrates the location of the single source position at the center of the survey with ray paths to a selected subset of receiver locations in the receiver grid. Each one of these rays defines a source-receiver path for both the $\mathrm{X}$ and $\mathrm{Y}$ oriented receiver positions from this single source position in the center of the survey. Each of these paths has its own sourcereceiver azimuth, the recorded data from this single east polarized source position gather of X-component receiver all these traced, organized by source-receiver offset, is shown in Figure 49 the equivalent interpreted reflecting horizon in the stacked data is identified. These data along with the Y (North) oriented shear component are then used to estimate the polarization of the reflector at the receiver position before the polarization correction is applied. This record shows a pre-stack offset vs. time gather line for X oriented source (a single location) and the $\mathrm{X}$ oriented receiver pairs. The event of interest is at $\sim 1100 \mathrm{msec}$, and it was selected to apply the polarization distortion correction. Note each 
trace has a different offset and azimuth associated with the shot 2479 . Also note that at the interpretation level; we see coherent reflections to offsets of $+/-4000$ meters, but little reflected energy beyond that offset. The depth to the reflector is about 600 meters, so this 4000 meters offset corresponds to an incident reflection angle of about $70^{\circ}$. 


\section{Source-Receiver offset (FEET)}

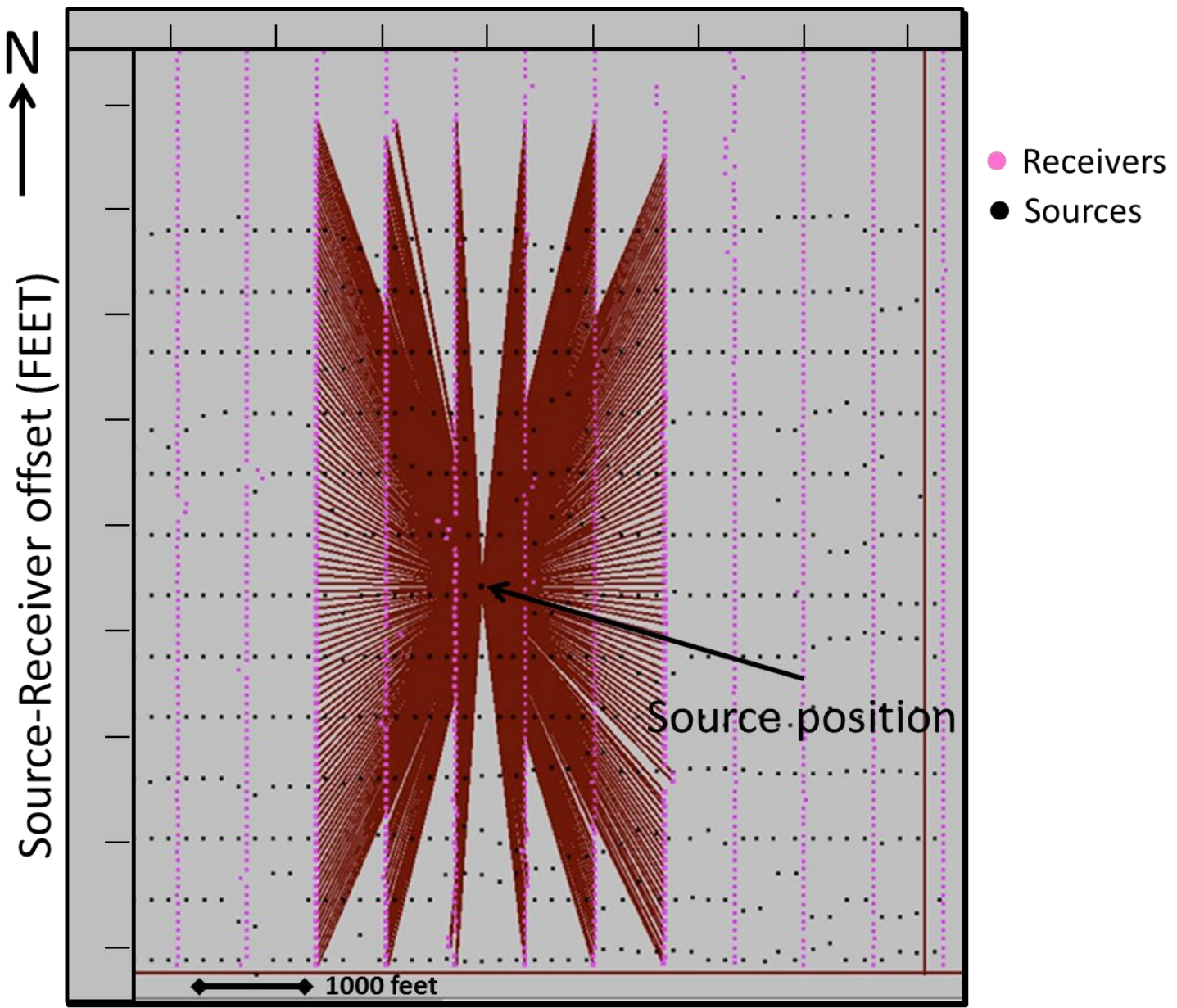

Figure 48: Single source position and orientation in the $\mathrm{X}$ and $\mathrm{Y}$ oriented receiver grid with a selection of associated $3 \mathrm{C}$ receiver locations. Note the wide range of azimuths and offsets for source-receiver picks associated with the single source position. In the total survey, many more source and receiver positions are available. The red lines are the in-line direction between the source and equivalent receiver used to calculate azimuth. The maximum sourcereceiver offset in this set is about 3500 feet. 


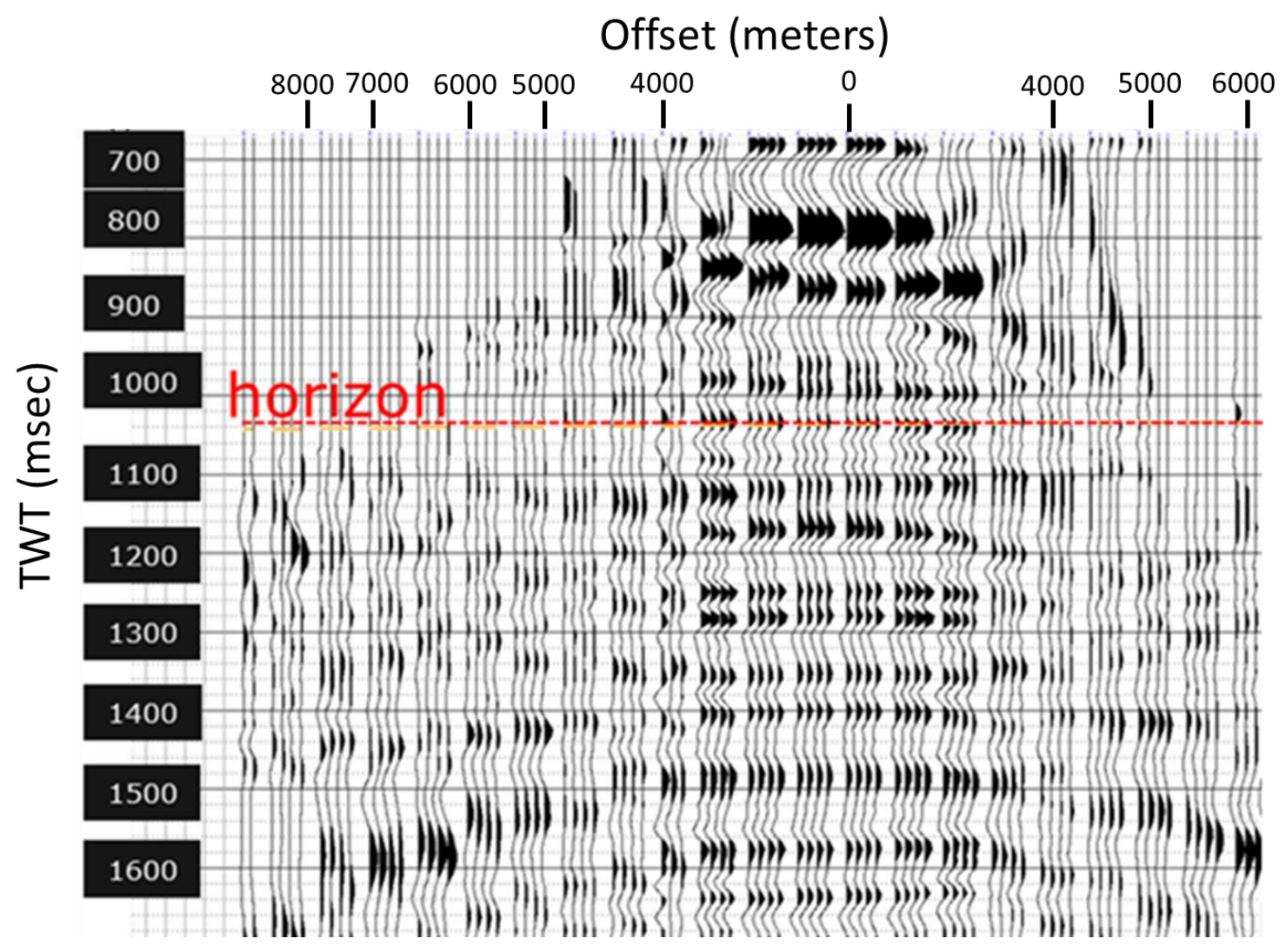

Figure 49: Single source position (source) gathers from the single $\mathrm{X}$ recorded trace (east) oriented shear source shown in Figure 48and recorded by X (east) oriented receiver organized by source-receiver offsets. Note the single source data we sorted by offset so the polarization correction can be applied. Each trace has a different source-receiver azimuth. The reflector (strong on the stacked volume) at $1050 \mathrm{msec}$ on the stacked section is identified. The polarization correction will be applied to this reflector, so this offset represents some large reflection incidence angles. Note that the reflection quality is best at source-receiver offset up to about $4000 \mathrm{~m}$. The reflector is at a depth of about 600 meters. 


\section{Polarization Observations and Correction Black Bear 3D 9C Seismic Data}

I now continue the analysis of the pre-stack seismic data to hopefully, understand how the polarization distortion and how the polarization correction works, on a portion of Black Bear 3D 9C data set. This includes applying the same correction that was applied to the $3 \mathrm{D}$ synthetic survey models for an isotropic medium over an anisotropic medium presented earlier. Utilizing the orientation of the anisotropy estimated from the VSP and dipole log analysis in the regionally "nearby" Texaco Brady Ranch 1-5 well, it appears that the entire section above the top of the Mississippian unconformity displays strong azimuthal anisotropic (we will assume HTI) with an HTI axis of symmetry $180^{\circ}(\mathrm{N}-\mathrm{S})$. (See the VSP discussion, shown in (Figure 36.A). This $180^{\circ}$ fracture - strike orientation inferred from the Brady well is quite consistent above the top of Mississippian unconformity. Ohanian and Beckham, (1992) and Beckham (1996) did considerable analysis of the data at the Sycamore level but that stratigraphic levels not included in this analysis. We must keep in mind that this region is quite complex tectonically and fracture orientations may vary considerably over rather short distances.

From the Brady well data, we may assume that this tectonic regime displays considerable shallow azimuthal anisotropy. From my discussions in Chapter 2, as illustrated in Figure 20, we note that the observed polarization of a shear wave takes on a polarization defined by the last medium it traveled through in this case the shallow medium. From earlier discussion about the geological setting we know it's a region of high tectonic activity. Thus, if there is shallow azimuthal anisotropy, it will control the

observed polarization of the vertically propagating shear wave. This effect limited my model examples in Chapter 2 to an isotropic layer over HTI layer. 
As a preliminary pass at the polarization analysis of the shear-wave reflections from the selected shallow horizon, consider the actual observed polarizations of the shear-wave reflections recorded from the X-oriented shear source are (shown in Figure 50). In this figure we see a remarkably consistent polarization of the reflected waves across the survey area oriented approximately north $45^{\circ}$ east. This orientation is not consistent with that observed in the entire shallow section (both above and below the depth of the reflecting layer) at the Brady Ranch 1-5 well location some 30 miles away. Further, the rather strong anisotropy at the Brady well is consistent with a consistent local polarization at that location. Keep in mind that the $45^{\circ}$ difference in azimuthal anisotropy at the Brady location and this survey location is not especially alarming. We certainly expect regional variations.

The blue crosses on Figure 50 represent the receiver locations. Both X (east) and Y (North) oriented receivers are used to determine the observed polarization of the reflected event. The length of the vectors indicates the amplitude of the recorded reflection, and the orientation of the vectors indicates the observed polarization. Keeping track of the polarization distortion associated with the reflection process and the polarization imposed by the shallow layer is very important in analyzing the polarization distortion issue imposed during the reflection process. In this case, the shallow layer defines the polarization no matter what the polarization at the reflection may be. Thus, the polarization distortion associated with the reflection process may be moot. Essentially all of the receiver locations observe purely S1 polarizations at the shallow section parallel to the fracture direction consistent with the presumed anisotropy of the upper medium of wave propagation.

Figure 51, shows the polarizations corrections (assuming some distortion) applied to the observed polarizations in (Figure 50) for a single shear source oriented due 123 
east (source at center and six receiver lines as shown in Figure 42) of the 3D 9C BlackBear survey. The length of the vectors indicates the amplitude of the recorded reflection, and the orientation of the vectors indicates the observed polarization. Note there are slight differences between the observed and corrected polarizations. Note: offset means the distance from the center at the source point. The similarity between the polarization after correction for assumed distortion and the observed polarization aligned with the HTI symmetry suggests that the polarization correction does "minimal" damage to the observed polarization. One difference between the observed polarization and the corrected polarization is all the receivers in the corrected polarization possibly correctly define the lower layer anisotropy because we have removed the distortion effect.

In Figure 52 we rotate the source polarization used in Figure 50 from due east to a north oriented source for a observed reflection polarization for a single shear source for the 3D 9C BlackBear survey from a single reflector at $1050 \mathrm{msec}$. Length of the vectors indicates the relative amplitude of the recorded reflection, and the orientation of the vectors indicates the observed polarization. For the case of no polarization distortion, the reflection polarization would be in the $\mathrm{Y}$ (north) direction. Note the observed fracture direction for east oriented source and north oriented source are the same because it's the same reflector level and the waves are propagating through the same near-surface layer. Now we apply the polarization distortion correction for a north oriented source (Figure 53) and the resultant polarization is oriented in the same direction as the observed polarization. Note the fractures are oriented $30^{\circ}$ northeast-southwest. Due to the difference in north source direction here Figure 50 we see slight difference in the observed polarization especially at far offsets. Note at offsets $\geq 4000 \mathrm{~m}$ the fracture direction of the reflected polarization is better determined than a source due east (Figure $50)$. 
Figure 53 shows the corrected polarization applied to the observed polarization distortions (Figure 52) for a single shear source oriented due north (source at center and six receiver lines as shown in Figure 42) of the 3D 9C BlackBear survey for a single reflector $500 \mathrm{msec}$. For the case of no polarization distortion and an isotropic shallow medium, the reflection polarization would be in the $\mathrm{X}$ (east) direction. Note the fractures are oriented $30^{\circ}$ northeast-southwest. Note at higher percentages of anisotropy, the resultant reflected polarization map still correctly defines the HTI anisotropy (as shown). This indicated that no matter how the source is oriented in the east or north direction we can properly define the fracture. At far offsets the fracture direction can be identified but it may be more difficult as seen in Figure 50 and Figure 52. Note Offset means the distance from the center at the source point.

Basically, for a shear wave propagating vertically through any HTI anisotropic material will be split into S1 and S2 polarizations regardless of polarization of the original wave. The upward propagating shear-wave reflections that impose the observed polarizations the observed wave regardless of the source polarization, is always the same defined by the HTI layer. To readily observe the actual polarization distortion caused by the reflection process would require a survey with an isotropic upper layer. Thus, I failed to experimentally observe the polarization distortion caused by the reflection process itself. This is due to the rather strongly HTI anisotropy of the entire shallow section. Nonetheless we can still learn much from these rather good quality shear-wave data.

The corrections applied to the observed data shown in these Figures 52 and 53 demonstrated results essentially equivalent to the observed polarization. Recall that the observed polarizations appear to be identical to that of the shallower sediments and thus controlled solely by the HTI anisotropy of the shallow sediments. This result is quite interesting in that the reflection distortion corrections appear to do no harm to the actual 
sediment induced polarization. Thus, the correction did the patient no harm. Possible reasons for this include the high levels of HTI anisotropy in the reflecting medium or we are not imaging a isotropic over anisotropic medium. Hence we need to apply a layer stripping approach to the seismic data which is not in the scope of this project. 


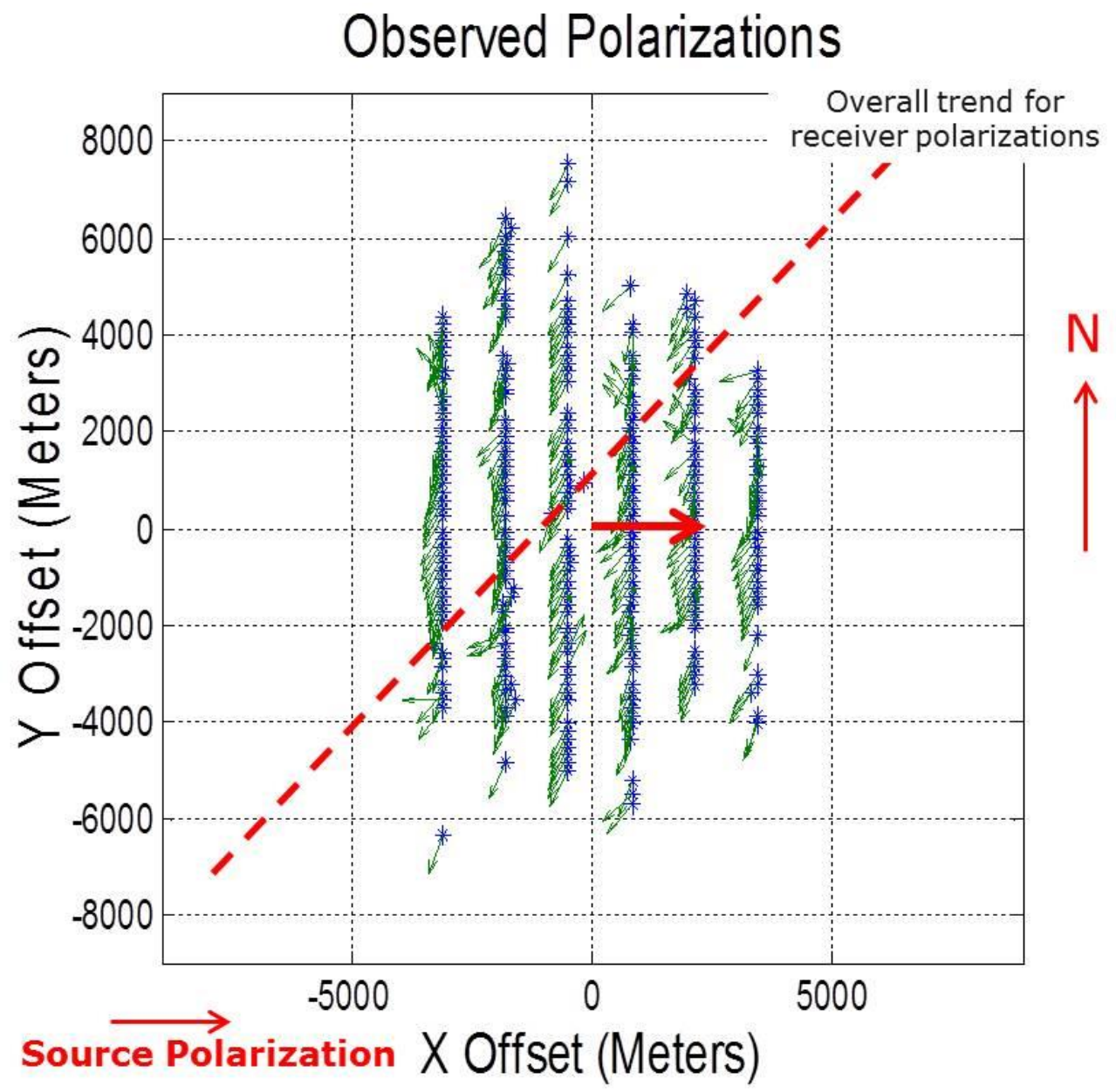

Figure 50: Observed reflection polarization for a single shear source oriented due east (source at center and six receiver lines as shown in Figure 42) of the 3D 9C BlackBear survey from a single reflector at $1050 \mathrm{msec}$. The blue crosses represent the receiver locations. Both X (east) and Y (North) oriented receivers are used to determine the observed polarization of the reflected event. The length of the vectors indicates the amplitude of the recorded reflection, and the orientation of the vectors indicates the observed polarization. Due to the polarity reversal for shear-wave reflections at or normal incidence where there is no polarization distortion, the reflection polarization would be in the $\mathrm{X}$ (east) direction for purely isotropic material. Note the regional fracture orientation is expected to be $30^{\circ}$ northeastsouthwest. Source-receiver offset the distance from the source point at center, to the receiver location. Essentially all of the receiver locations observe purely S1 polarizations at the shallow section parallel to the fracture direction consistent with the presumed anisotropy of the upper medium the wave propagate through. 


\section{Corrected Polarizations}

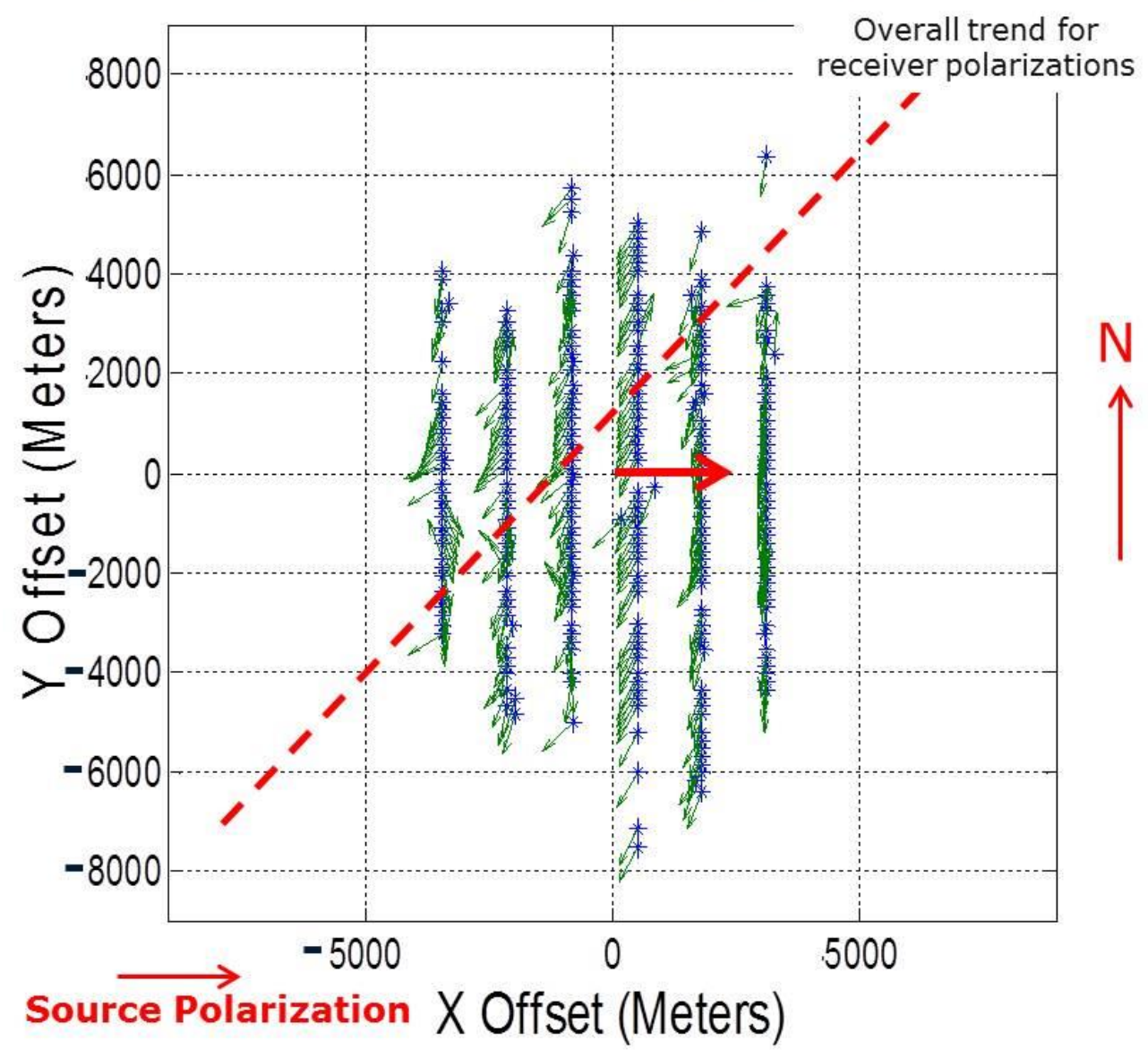

Figure 51: Corrected polarizations applied to the observed polarization distortions for a single shear source oriented due east (source at center and six receiver lines as shown in Figure 42) of the 3D 9C BlackBear survey. Blue crosses represent the receiver locations. The length of the vectors indicates the amplitude of the recorded reflection, and the orientation of the vectors indicates the observed polarization. Note the similarity between the observed and corrected polarizations. Note Offset means the distance from the center at the source point. The similarity between the polarization after correction for assumed distortion and the observed polarization aligned with the HTI symmetry suggests that the polarization correction does "minimal" damage to the observed polarization. 


\section{Observed Polarizations}

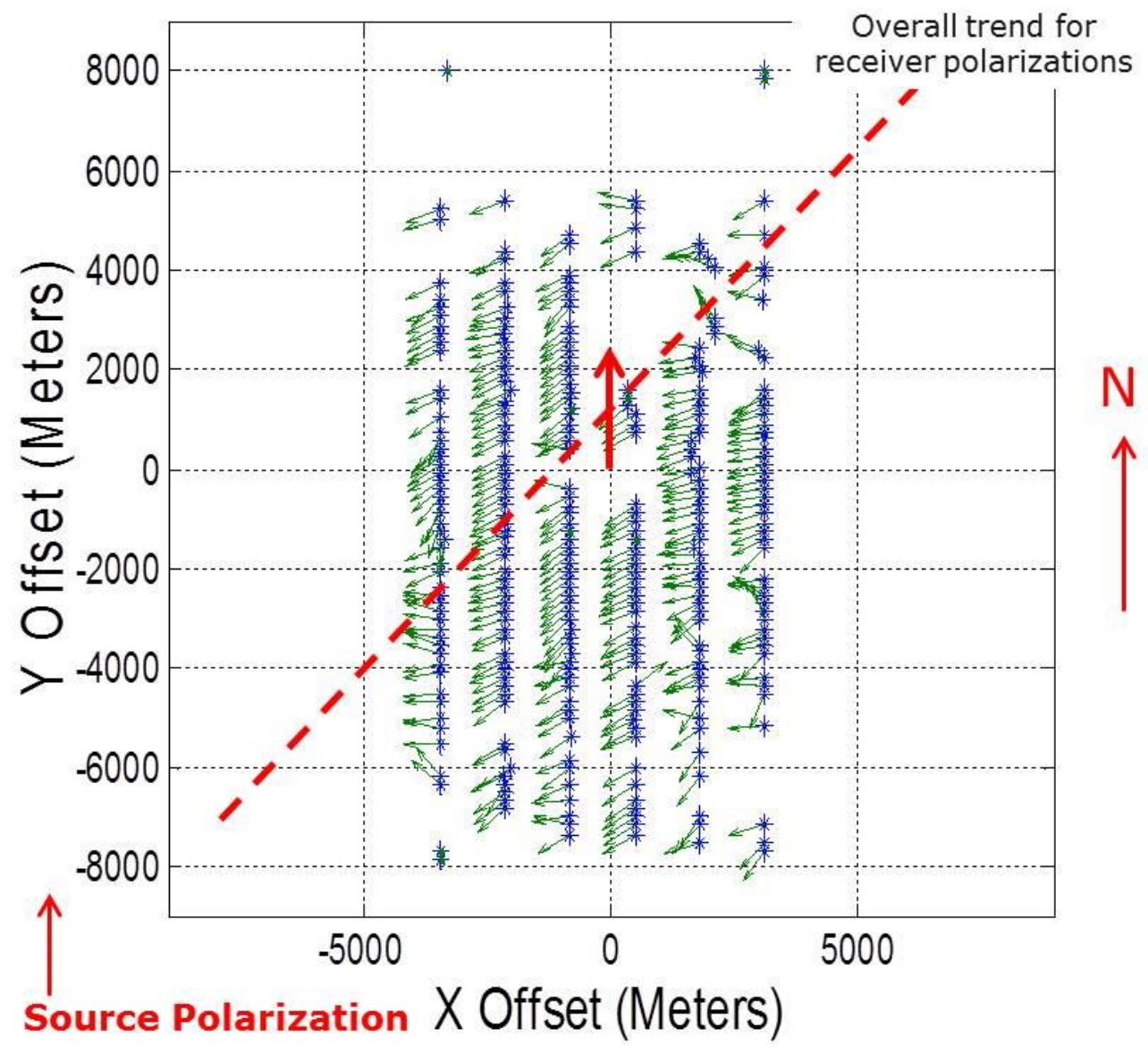

Figure 52: North oriented source for a observed reflection polarization for a single shear source oriented due north (source at center and six receiver lines as shown in Figure 42) of the 3D 9C BlackBear survey from a single reflector at 500 msec. The blue crosses represent the receiver locations. Length of the vectors indicates the amplitude of the recorded reflection, and the orientation of the vectors indicates the observed polarization. For the case of no polarization distortion, the reflection polarization would be in the $\mathrm{X}$ (east) direction. Note the fracture direction for east oriented source and north oriented source are the same because it's the same reflector level. Source-receiver offset is the distance from the center at the source point. Due to the difference in source direction than Figure 50 we see slight difference in the observed polarization. Note at offsets $\geq 4000 \mathrm{~m}$ the fracture direction of the reflected polarization is better determined than a source due east (Figure 50). 


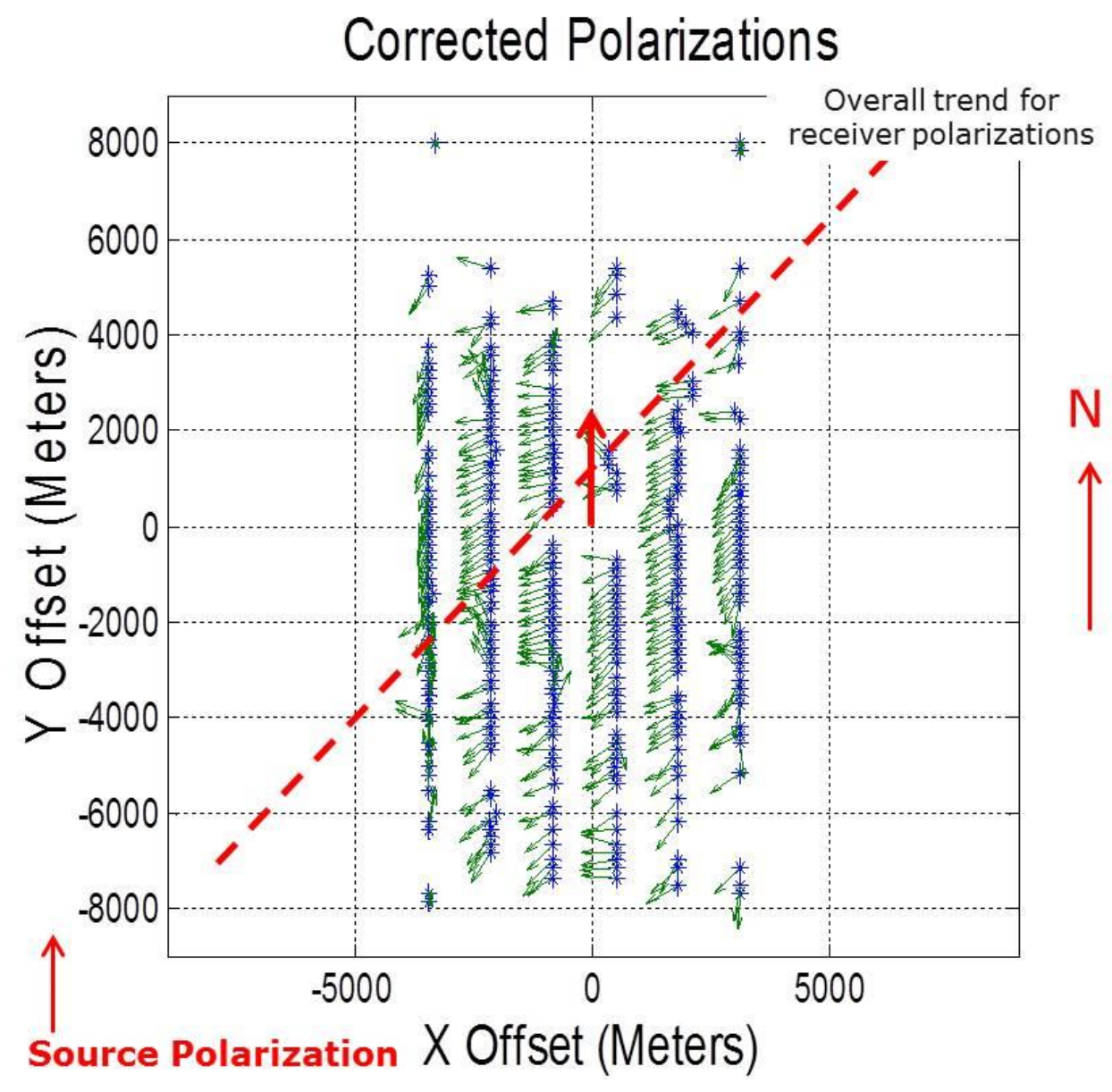

Figure 53: Corrected Polarization applied to the observed polarization distortions for a single shear source oriented due north (source at center and six receiver lines as shown in Figure 42) of the 3D 9C BlackBear survey for a single reflector $500 \mathrm{msec}$. The blue cross represents the receiver locations. Both $\mathrm{X}$ (east) and Y (North) oriented receivers are used to determine the actual polarization of the reflected event. The length of the vectors indicates the amplitude of the recorded reflection, and the orientation of the vectors indicates the observed polarization. For the case of no polarization distortion, the reflection polarization would be in the $\mathrm{X}$ (east) direction. Note the fractures are oriented $30^{\circ}$ northeast-southwest. Note at higher percentages of anisotropy (seen in the Brady-1 well), the resultant reflected polarization map still correctly defines the HTI anisotropy (as shown). This indicated that no matter how the source is oriented in the east or north direction we can properly define the fracture. At far offsets the fracture direction can be identified but it may be more difficult as seen in Figure 50 and Figure 52.. Note Offset means the distance from the center at the source point. 


\section{CHAPTER 4: ALFORD ROTATION \\ Multicomponent Data}

Controlled source exploration seismology is the specific area of geophysics that this study falls under. By controlled sources, we include control of not only location and strength of the source, but also the polarization of the particle motion for both P and S wave generation. This control of the source parameters is designed to ultimately focus on fracture characterization using analysis of propagating seismic waves. Fracture characterization using seismic data was discussed in Chapter 3, utilizing to the Black Bear survey to demonstrate the effects of the anisotropic fractured medium on the seismic wave propagation, specifically the polarization of shear-waves. Thus, independent three component source and three component receiver orientations taken at a source and receiver location provides a total of nine components, or combinations of recorded data, often referred to as $9 \mathrm{C}$ (nine component) data. The $3 \mathrm{D}$ designation refers to the three dimensional subsurface volume sampled, which commonly leads to the 3D-9C data designation. These multicomponent seismic data incorporate all 9 components to record compressional data and two orthogonal polarizations shear wave data. The orientation of the particle motion (polarizations) and the wave propagation directions are further delineated (Tatham and McCormack, 1991) to provide analysis of the anisotropic texture character of the medium the waves propagate through.

Multicomponent seismic reflection data (9C-3D) are acquired using two

orthogonal horizontal (predominantly shear) and one vertical (predominantly compressional) source polarization recorded with two orthogonal horizontal and one vertical geophones orientation. Horizontal (shear-wave) sources and geophones are commonly oriented in arbitrarily orthogonal X and Y directions on Earth's surface. These 
$\mathrm{X}-\mathrm{Y}$ surface coordinates are referred to as field or survey coordinates. In this geometric survey, designation, we acquire four horizontal source data sets. The X-source Xreceiver, $\mathrm{X}$-source $\mathrm{Y}$-receiver, $\mathrm{Y}$-source $\mathrm{X}$-receiver, $\mathrm{Y}$-source $\mathrm{Y}$-receiver traces.

Keep in mind that each of the three receiver components implicitly records a mixture of SH, SV, and P-waves. The degree of mixing between these elements depends on source-receiver azimuth, as well as the deviation of the propagation path from vertical. This makes shear-wave data processing, signal recovery, and interpretation rather complex in the original survey coordinates because of this spatially variable mixing of wave modes (Simmons et al., 2001).

Analyzing shear-wave data may be simplified by trigonometrically rotating recorded data in individual source-receiver pairs into a radial and transverse coordinate system relative to the individual source-receiver azimuth for each source - receiver pair. The radial-source radial-receiver component then contains predominantly SV waves and $\mathrm{P}$ waves, while the transverse-source transverse-receiver component contains predominantly SH waves in an isotropic flat-layered earth (see chapter 1).

A 9-C-3-D data set acquired with an orthogonal field configuration consisting of $\mathrm{X}-\mathrm{Y}$ source and receiver locations and polarizations exemplifies the azimuthal (sourcereceiver) dependence of survey coordinate data. At all other source-receiver azimuths other than solely $\mathrm{X}$ and $\mathrm{Y}$, the four horizontal survey coordinate data sets contain a mixture of $\mathrm{SH}, \mathrm{SV}$, and $\mathrm{P}$-waves in varying proportions that are azimuth dependent. In trigonometrically resolved radial-transverse oriented coordinates, SV data are contained on Source Radial, Receiver Radial components and SH information is contained on Source Transverse, Receiver Transverse data at all azimuths, and energy in the crossterms is minimized. 
Simmons (2001) demonstrated the effectiveness of separating SV and SH components by trigonometrically rotating survey coordinates into radial-transverse coordinate (Figures 54 and 55 respectively). The prestack data from an entire survey are binned in where prestack data are sorted into $10^{\circ}$ azimuth bins and $100-\mathrm{ft}$ offset bins within each CMP location. These bin-gathered data can then be corrected and summed into interpretable data volumes and then summed as a function of source-receiver azimuth and offset for each CMP location in the entire survey. In the bin gathers (Figure 54) at a azimuth $0^{\circ}$, crossterms energy from the $\mathrm{Y}$-oriented source, $\mathrm{X}$-oriented receiver traces and $\mathrm{X}$-oriented source, $\mathrm{Y}$-oriented receiver traces is minimal. Note that at a sourcereceiver azimuth of $90^{\circ}$ equivalent to (2-D acquisition in the $\mathrm{X}$ direction), Y-oriented source, $\mathrm{X}$-oriented receiver and $\mathrm{X}$-oriented source, $\mathrm{Y}$-oriented receiver energy in the recorded traces is again at a minimum, but now the SH data appear on X-oriented source, $\mathrm{X}$-oriented receiver traces and the $\mathrm{SV}$ data appear on Y-oriented source, Y-oriented receiver traces. As the source-receiver azimuth increases from zero, crossterm amplitudes increase and reach a maximum at an azimuth of $45^{\circ}$. There is little visible difference between the four survey coordinate data sets at $40^{\circ}$ and $50^{\circ}$. The large difference in the shear wave first-arrival times between $\mathrm{SH}$ and $\mathrm{SV}$ traces suggests the presence of a medium containing strong azimuthal anisotropy (Simmons et. al, 1999).

This example illustrates the effectiveness of rotating all polarizations to $\mathrm{SH}-\mathrm{SH}$ interpretation for interpretation. The $\mathrm{SH}-\mathrm{SH}$ data, with minimal effect of mode conversion to P-waves and no zero crossing in the reflectivity at relatively small angles of incidence are probably the preferred data for analysis of geological structure. The rotation process to SH-SH however, abandons the inherent polarization information in the shearwave data that may be used for interpretation of textural characterization (such as fracturing and azimuthal anisotropy) of the subsurface volume. The basic thesis of this 
dissertation is that we can correct for and utilize polarization information in interpretation. 


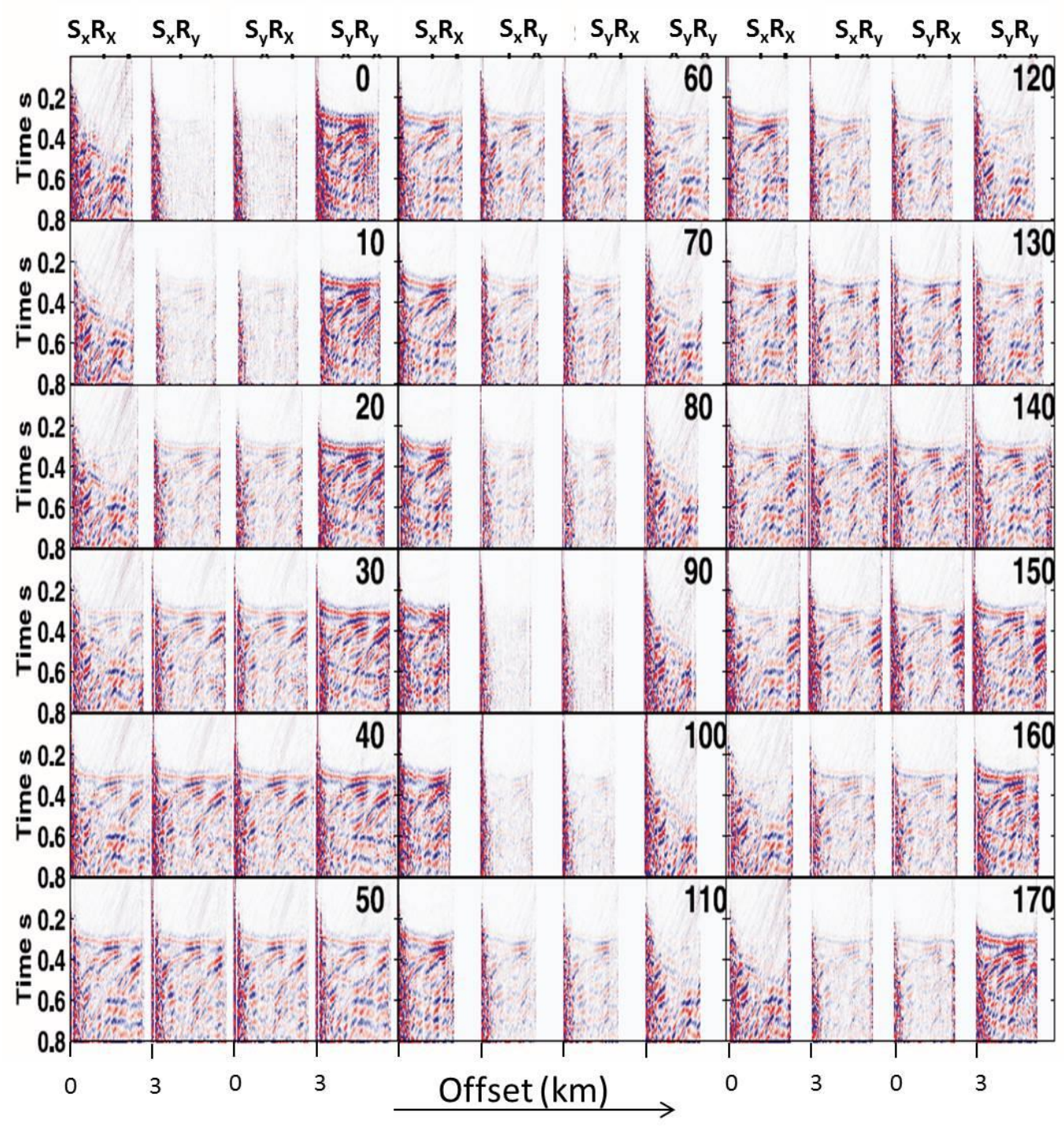

Figure54.CMP gathers in survey coordinates as a function of source-receiver azimuth. Each set of 4 panels represent polarization of source-receiver pairs: $\mathrm{SxRx}-$ $\mathrm{X}$ oriented source X-oriented receiver, SxRy $-\mathrm{X}$ oriented source $\mathrm{Y}$ oriented receiver, SyRx - Y oriented source X oriented receiver, SyRy - Y oriented source Y oriented receiver. The data are full-prospect "super gathers" (sums of all CMP bin gathers from the entire survey) in survey coordinates as a function of source-receiver azimuth. Data are displayed in two-way reflection time in seconds and offset, in $\mathrm{km}$. Note each panel has an offset from 0 to $3 \mathrm{~km}$ and it is NMO corrected at a constant shear-wave velocity. Source receiver offset increases from $0-3 \mathrm{~km}$ to the right in each subpanel. Source-receiver azimuth is noted in the upper right of each panel and offset, in $\mathrm{km}$ is indicated at the bottom of the panels(Simmons, 2001). 


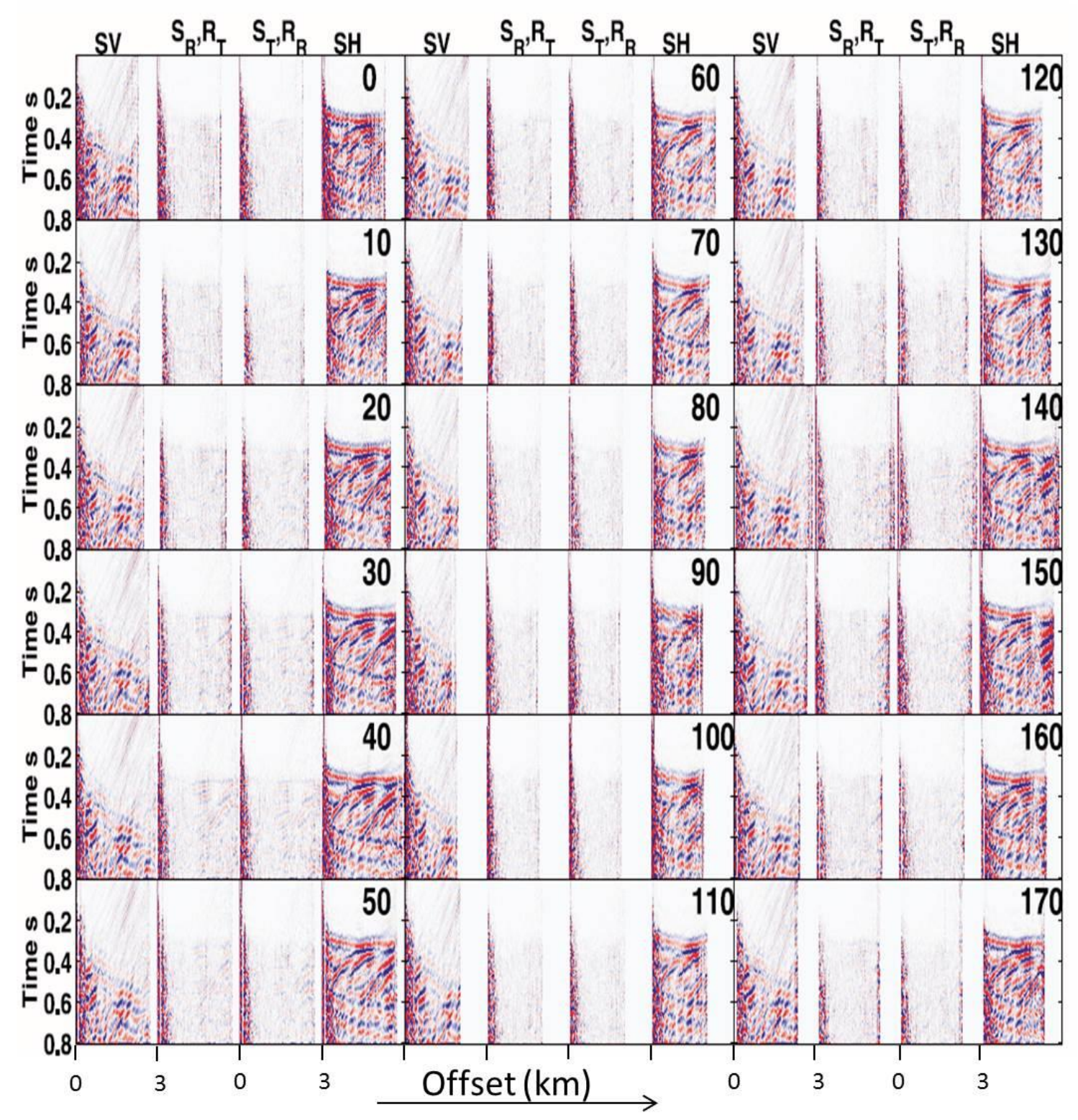

Figure 55: Data from Figure 54 after rotation into radial and transverse components for each source-receiver pair. The cross-term energy is at minimum and the $\mathrm{SV}$ and $\mathrm{SH}$ data is isolated in the $\mathrm{S}_{\mathrm{R}}-\mathrm{R}_{\mathrm{R}}(\mathrm{SV})$ and $\mathrm{S}_{\mathrm{T}}-\mathrm{R}_{\mathrm{T}}(\mathrm{SH})$ components. The data are full-prospect "super gathers" (sums of all CMP bin gathers from the entire survey) in survey coordinates as a function of source-receiver azimuth. Data are displayed in time and they are NMO corrected. Note each panel has an offset from 0 to $3 \mathrm{~km}$. Source receiver offset increases from 0 to $3 \mathrm{~km}$ to the right in each subpanel. Source-receiver azimuth is noted in the upper right (Simmons, 2001). 


\section{Rotating SURVEY COORdinAteS}

Orientation of a coordinate system for polarization analysis based on actual subsurface conditions is crucial to the goal of characterizing azimuthal anisotropy. One objective of multicomponent 9C-3D seismic surveys is to estimate azimuthal anisotropy to characterize fracture parameters. The surveys however, are generally designed with limited previous knowledge of the orientation of fracture systems in the subsurface. Hence, actual data acquisition geometries result in an acquisition coordinate system I refer to as survey; (or field) coordinates (Figure 56). In land acquisition, the survey coordinate system may align with geographic orientations such as X (east) and $\mathrm{Y}$ (north). In Figure 56, the survey, or field coordinates are up for $\mathrm{X}$ and left for $\mathrm{Y}$.

As discussed, simply recording data in arbitrary $\mathrm{X}$ and $\mathrm{Y}$ receivers of a survey coordinate system defined by the acquisition geometry is not necessarily optimum to the analysis and interpretation of shear waves polarizations for analysis of azimuthal anisotropy (Alford, 1986; Crampin, 1986). An early step in the analysis procedure has often been used to rotate the observed S-wave reflection polarizations to source to receiver oriented radial and transverse coordinates (Figure 56), where radial and transverse components are defined by the direction or azimuth between the source and receiver locations. This coordinate system coincides with true SV and SH polarizations and may be optimum for analysis of isotropic materials. In anisotropic cases, such as HTI symmetries of azimuthal anisotropy that result from vertical fractures, it is preferred to orient the shear waves polarizations into parallel (S1, fast) and perpendicular (S2, slow)

orientation relative to the axis of symmetry (Figure 56) describing the HTI anisotropy associated with the subsurface media. Such a rotation analysis is required to determine the $\mathrm{S} 1$ and $\mathrm{S} 2$ orientations in the substrate. 
Now we apply the process of trigonometrically rotating data in recorded survey coordinates into data oriented in radial-transverse (relative to source - receiver azimuth) coordinates. Initially, we rotate four pairs of $(\mathrm{X}, \mathrm{Y})$ components in shot (or receiver) gathers to radial and transverse coordinates defined for $\left(S_{R}-R_{R}, S_{R}-R_{T}, S_{T}-R_{R}, S_{T}-R_{T}\right)$ source-receiver trace pairs associated with each source-receiver pair. After rotation, the $\mathrm{SV}$ data are all on the radial-radial oriented traces and the $\mathrm{SH}$ data to the transversetransverse oriented traces. The rotation process computes the azimuth angle $(\Phi)$ of the angle between the shot and the receiver location. Then it applies the following rotation operation to output new traces.

$$
\mathrm{X} \text { (output) }=\cos (\Phi) \times \mathrm{X} \text { (input) }+\sin (\Phi) \times \mathrm{Y} \text { (input) }
$$

$$
\mathrm{Y}(\text { output })=\sin (\Phi) \times \mathrm{X}(\text { input })-\cos (\Phi) \times \mathrm{Y} \text { (input) }
$$

where $\mathrm{X}$ (input) and $\mathrm{Y}$ (input) are the unrotated input $\mathrm{X}$ and $\mathrm{Y}$-oriented traces. After a scan of rotations we can estimate through a range of azimuths $\Phi$, the natural fracture orientation for the HTI medium which is important in understanding fracture characterization and its orientation. This scan process was introduced by Alford (1986) and his colleagues. 

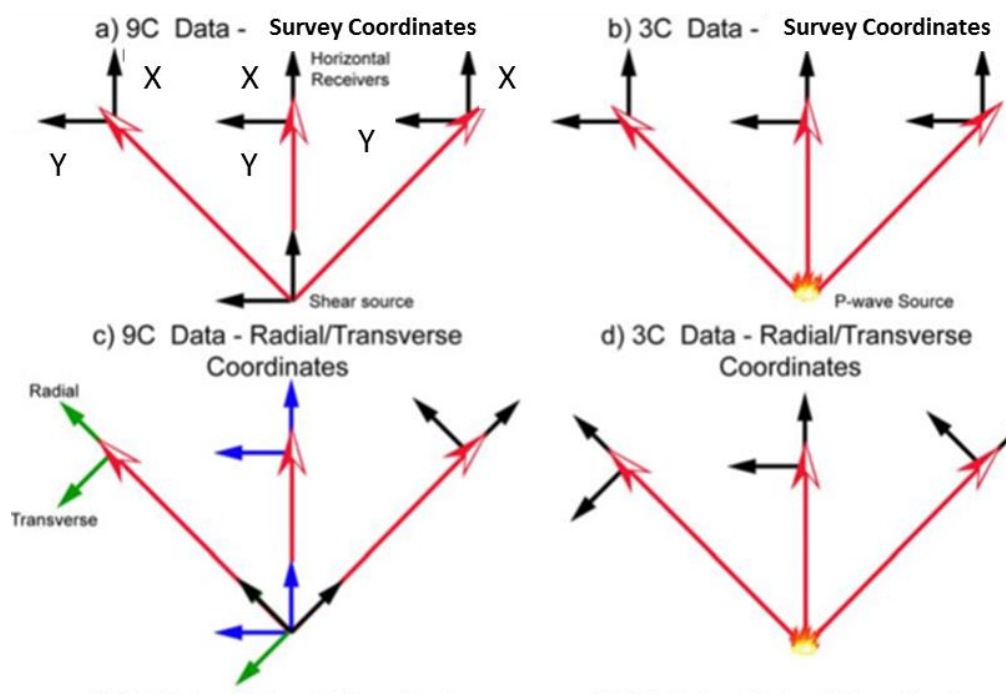

d) 3C Data - Radial/Transverse Coordinates
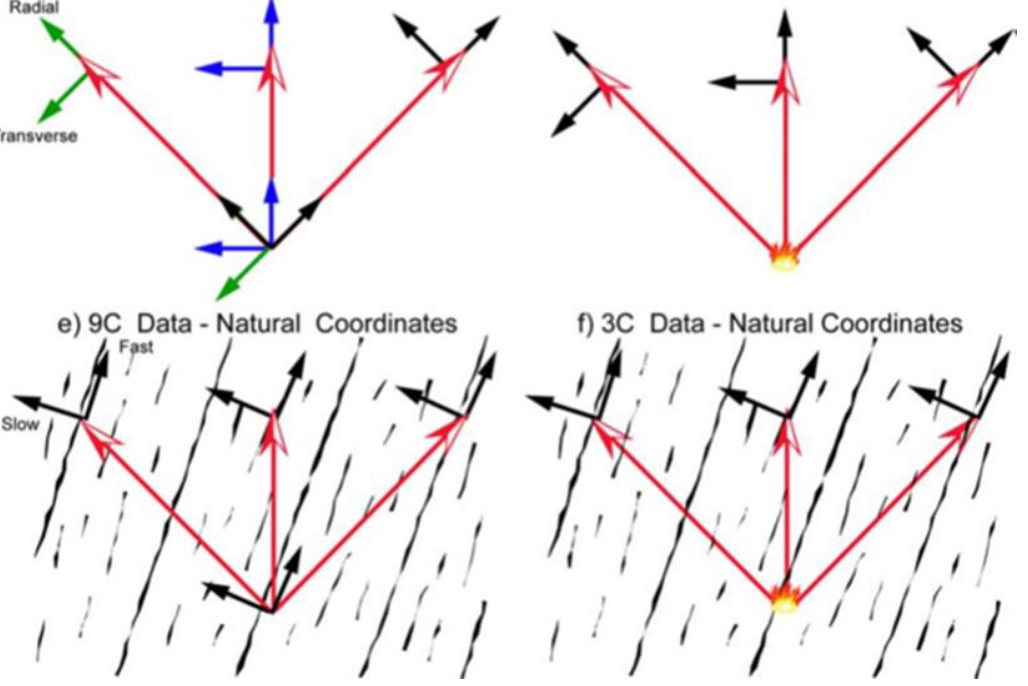

Figure 56: Description of coordinate systems used in rotation analysis of horizontal components for both 9C (3-source and 3-receiver components) and 3C (1 source and 3 receiver components) acquisition. Panels (a) and (b) show a single source position and three receiver positions in plan view, with the red arrows showing source-receiver azimuthal paths for both the 9C case (a) and the 3C P-SV mode converted case (b). Note that X is upward and $\mathrm{Y}$ is to the left this particular "survey-parameter" orientation. For the $9 \mathrm{C}$ case both source and receiver polarizations are aligned in uniform $\mathrm{X}$ and $\mathrm{Y}$ directions defined by the acquisition survey parameters. The $3 \mathrm{C}$ case is similar but with a compressional (vertical, or Z, polarization) source. Panels (c) and (d) illustrate the resultant source and receiver orientations after trigonometric rotation of the recorded horizontal components to radial and transverse coordinate. For the $9 \mathrm{C}$ case (c) the source and receiver polarizations are both rotated to the azimuth direction. For the $3 \mathrm{C}$ case $(\mathrm{d})$ where there are no horizontal source components, only the receiver polarizations are defined by the source-receiver positions, rotated to radial and transverse components. In figures (e) and (f) the underlying background lines represent parallel joints or cracks typical of an HTI (transverse isotropy) medium. Where appropriate the horizontal source and receiver polarizations are aligned in preferential S1 and S2 orientations of the fractures (Gumble, 2006). 


\section{Vertical Incidence Direct Shear Waves and Alford Rotation}

Alford (1986) proposed a matrix multiplication technique for implementing the rotation of shear-wave traces recorded with 2 horizontal components of sources and two horizontal components of receivers from survey acquisition coordinates to any arbitrary coordinate orientation for the case of zero source- receiver offset (vertical incidence). This matrix method of rotation leads to a scanning procedure over all angles of incidence to determine the natural coordinate system of associated HTI symmetry. This coordinate system is then used as a proxy for orientation and the natural fracture direction by estimating the rotation angle that maximizes the ratio of the principal (X-X, Y-Y) terms to the energy of the off diagonal (X-Y, Y-X) components (Alford, 1986). Alternatively the angle may be determined by a visual scanning technique, originally conceived for small data sets. Later automated modifications have been made for larger data sets. The limitation of the Alford's rotation is that it is only valid for application to vertically propagating (1D) shear-wave data. However it has been commonly used in industry practice and applied to CMP stack of multi-offset surface seismic data sets.

Following the earlier discussion of separating multicomponent S-wave data with direct shear sources and receivers into four sets of horizontal components then applying a trigonometric rotation algorithm, the natural orientation of azimuthal anisotropy (assumed to be HTI anisotropy of a transversely isotopic medium) can be estimated by scanning through a complete range of possible polarizations of the reflected shear waves.

The four "components" in this case are the components defined by the following combinations of sources and receivers:

1) $X$ source to $X$ receiver,

2) $X$ source to $Y$ receiver 
3) $Y$ source to $X$ receiver

4) $Y$ source to $Y$ receiver

\section{Alford Rotation}

The fundamental solutions, or the four traces of post-rotated seismic traces associated with 2 horizontal sources and 2 horizontal receivers computed in a matrix representation, are given by the following equation:

$$
\underline{U}(\theta)=\underline{R}(\theta) \underline{V}(\theta) \underline{R}^{T}(\theta)
$$

where

$$
\underline{R}(\theta)=\left[\begin{array}{cc}
\cos \theta & \sin \theta \\
-\sin \theta & \cos \theta
\end{array}\right]
$$

and

$$
\underline{V}(\theta)=\left[\begin{array}{cc}
\left(\cos ^{2} \theta u_{11}+\sin ^{2} \theta u_{22}\right) & 0.5 \sin 2 \theta\left(u_{11}-u_{22}\right) \\
0.5 \sin 2 \theta\left(u_{11}-u_{22}\right) & \left(\sin ^{2} \theta u_{11}+\cos ^{2} \theta u_{22}\right)
\end{array}\right]
$$

where $\mathrm{u}_{\mathrm{ij}}$ represents the solutions of the shear components in the natural HTI coordinate system defined by the angle $\theta$. Combining these terms:

$$
\underline{U}(\theta)=\left[\begin{array}{cc}
\left(\begin{array}{c}
\cos ^{2} \theta v_{11}+\sin ^{2} \theta v_{22} \\
+.5 \sin 2 \theta\left(v_{21}-v_{12}\right)
\end{array}\right) & \left(\begin{array}{c}
\cos ^{2} \theta v_{12}+\sin ^{2} \theta v_{21} \\
+.5 \sin 2 \theta\left(v_{22}-v_{11}\right)
\end{array}\right) \\
\left(\begin{array}{c}
\cos ^{2} \theta v_{21}+\sin ^{2} \theta v_{12} \\
+.5 \sin 2 \theta\left(v_{22}-v_{11}\right)
\end{array}\right) & \left(\begin{array}{c}
\cos ^{2} \theta v_{22}+\sin ^{2} \theta v_{11} \\
+.5 \sin 2 \theta\left(v_{21}-v_{12}\right)
\end{array}\right)
\end{array}\right]
$$

where $\underline{V}$ are the solutions of the recorded shear components of the original data set.

The determination of the desired angle of rotation $\theta$ (and hence the principal axis of anisotropy) can be implemented in a variety of ways. For example; 1) through an 
iterative application of the rotational algorithm incrementing the rotation angle and visually inspecting the resultant rotation to find the maxima of the diagonal components and minima of the off diagonal components of the matrix; 2) through the same aforementioned iteration technique, while numerically estimating, the maxima and minima amplitude (the minima are generally more stable objectives for numerical estimation) or, 3)by maximizing the ratio between diagonal and off diagonal elements, potentially a more robust method. The alternative method would consist of a combination of numerical analysis and visual confirmation. Approximation and verification can also be made by organizing the original radial and transverse components into prestack azimuth gathers in bins sorted by a practical set of azimuth i.e., 10 degree bins. Then observing changes in polarity, as the azimuth of polarity change is typically associated with the principal axis of the anisotropic medium (Gaiser, 1999).

\section{Polarization Correction to Isotropic Medium}

Expanding the earlier discussion of polarization correction for shear-wave reflections at large angles of incidences to include two orthogonal source receiver orientations, I extend the Alford (1986) rotation to non-normal angles of incidence by minimizing the polarization distortion upon reflection at all offsets for each direct source component. Recall that Alford rotation, as originally proposed, is strictly valid for only normal-incidence (zero-offset) data, where there is no distinction between SV and $\mathrm{SH}$ reflectivity because all particle motion is in a common horizontal plane. In the presence of HTI anisotropy, cross-term energy is produced when the two shear-wave polarizations are at an arbitrary angle relative to the principal axes of HTI symmetry of the propagation

and the reflecting medium. As a final step, simplicity, I address propagation in an 
isotropic over both an isotropic layer and a isotropic layer. Alford's rotation is appropriate for propagation in both isotropic and HTI but polarization is distorted for non-normal incidence angles in both the isotropic and anisotropic cases.

Historically Alford rotation has commonly been applied to data preprocessed including CMP stacked data in survey coordinates. Some of the processes were intended to allow use of all data in the anisotropic analysis. Thus, the proposed polarization modification for offset should ultimately provide a more grounded basis for azimuthal polarization analysis. Because applications of Alford's rotation as originally proposed to 3D shear wave data at non-normal incidence is invalid, I will apply the polarization distortion correction to the original data before applying Alford's rotation analysis to extend the rotation to non-normal angles of incidence (Simmons, 1999; Lynn, 1986). We will discuss results of the polarization correction to multi offset data in an isotropic over isotropic model to demonstrate the offset effect and the efficacy of the correction. I will then expand the application to an isotropic over HTI medium.

Let us first consider a synthetic model of an isotropic layer over isotropic layer using the same model described in Table 1 of Chapter 2. The simple isotropic synthetic models were generated using the Anivec software. Figure 57 shows the results of the original isotropic model where the crossterms contain no information because HTI anisotropy is not present to generate shear wave splitting. The source and receiver oriented in the $\mathrm{X}$ direction $(\mathrm{XX})$, the source oriented in the $\mathrm{X}$ direction and the receiver in the $\mathrm{Y}$ direction $(\mathrm{XY})$, the source in the $\mathrm{Y}$ direction and the receiver in the $\mathrm{X}$ direction $(\mathrm{YX})$, and finally the source and receiver in the $\mathrm{Y}$ direction (YY). The (XY) and (YX) records are named the cross-term elements, because the source and receiver are orthogonal to each other in this case. All of the reflection strength has been projected onto either the radial or transverse components, supposedly eliminating the reflection 
effect. However, the four-component rotation used is developed for normal incidence data Alford (1986).

The recorded profile is oriented in the $\mathrm{X}$ direction, with offset increasing in the $\mathrm{X}$ direction from the source location. The X-X profile is purely SV-SV data, and the Y-Y profile is purely SH-SH data. Note that the Y-Y data are for the receiver located at the origin and the profile is on the $\mathrm{X}$ axis $(\mathrm{y}=0)$ increasing $\mathrm{X}$ direction. The zero-crossing location identified in the $\mathrm{X}$-oriented source $\mathrm{X}$-oriented receiver traces (SV) was calculated as $20^{\circ}$, and the zero-crossing location identified in the Y-oriented source Yoriented receiver traces $(\mathrm{SH})$ was calculated as $40^{\circ}$. Note in the $\mathrm{X}-\mathrm{X}$ synthetic trace the amplitude decreases from zero $0^{\circ}$ to $20^{\circ}$, where it goes through the zero crossing. After the zero crossing and polarity change, the amplitude increases beyond the critical angle of $49^{\circ}$. The same occurs in the Y-Y synthetic data, but the traces go to zero amplitude at $40^{\circ}$. Recall that Alford rotation is not valid for non-normal angles of incidence. This is because as seen in Figure 57 the reflection coefficient for SV-SV and SH-SH are not the same for larger angles of incidence. Applying the amplitude corrections for SV and SH components to this profile (Figure 57.A) demonstrated the efficacy of this correction on the individual SV and SH components. 

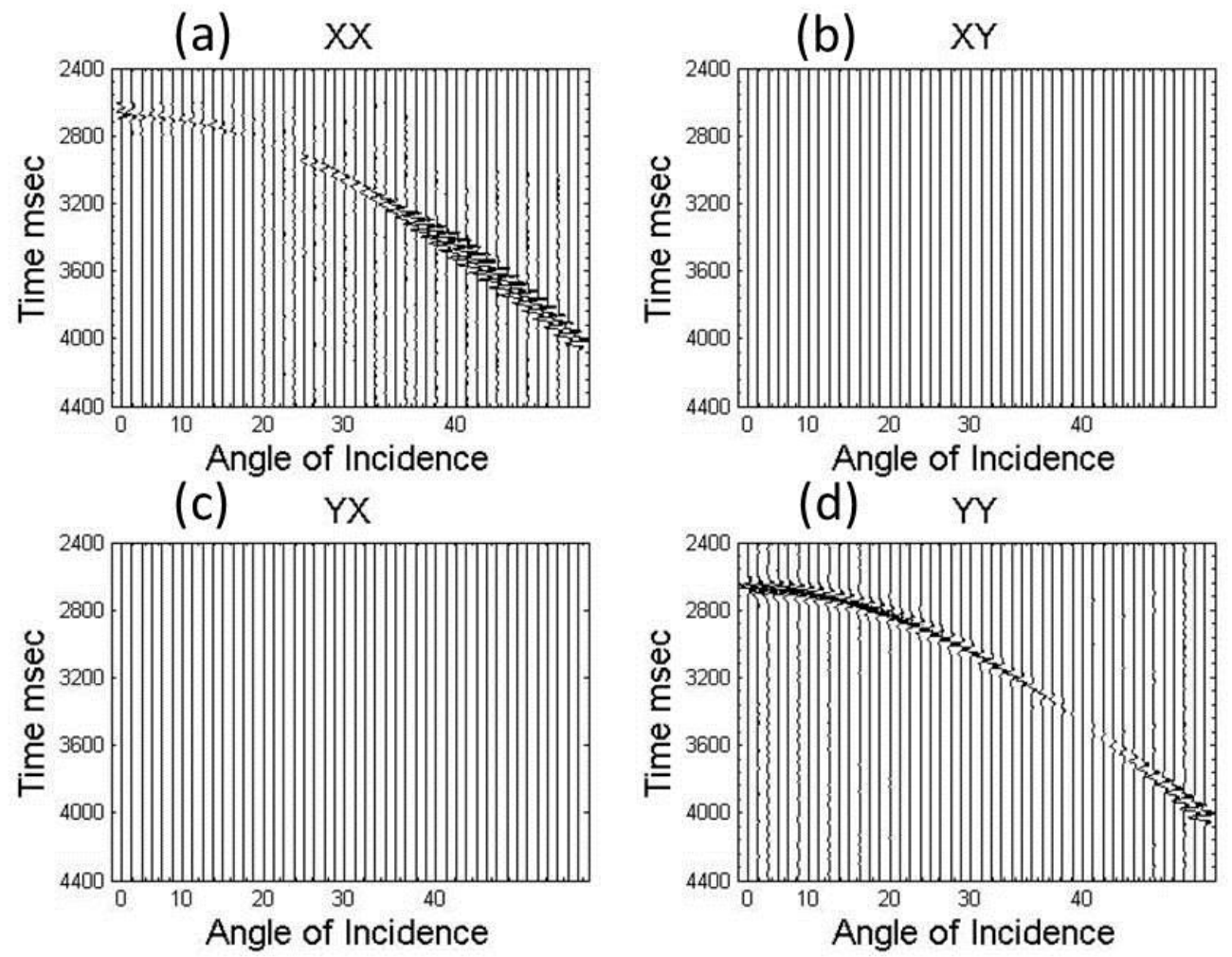

Figure 57: Synthetic data on a X-oriented time distance profile for a two layer isotropic model outlined in Table 1. Layer depth is at $2 \mathrm{~km}$. Horizontal axis is linear in offset, but labeled in equivalent angle of incidence. (a) data for the $\mathrm{X}$-source into the $\mathrm{X}$ receiver $(\mathrm{X}-\mathrm{X})$; (b) $\mathrm{Y}$-source $\mathrm{X}$ receiver; (c) $\mathrm{X}$-source, $\mathrm{Y}$ receiver; (d) Y-source, Y-receiver. Note that in the purely isotropic model, there is no energy on the $\mathrm{Y}-\mathrm{X}$ or $\mathrm{X}-\mathrm{Y}$ components. Also note the significant difference in reflection amplitudes with angle of incidence in the X-X (SV) and $\mathrm{Y}-\mathrm{Y}(\mathrm{SH})$ components. 

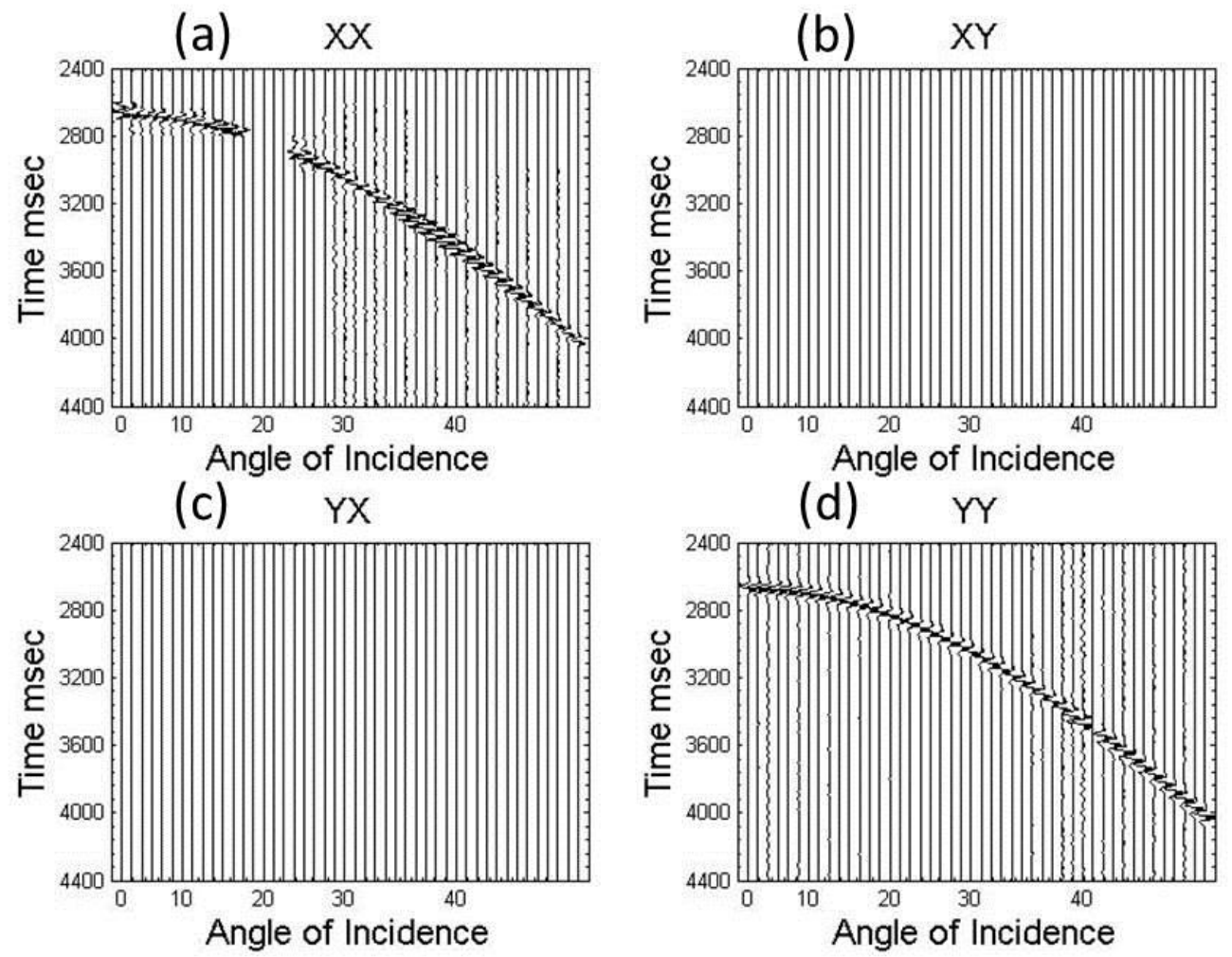

Figure 57. A: Correction for SV and SH amplitude applied to synthetic data from Figure 57 for an X-oriented time-distance profile with amplitude correction. Note the two layer isotropic model outlined in Table 1. Layer depth is at $2 \mathrm{~km}$. Horizontal axis is linear in offset, but labeled in equivalent angle of incidence. (a) data for the $\mathrm{X}$-source into the $\mathrm{X}$ receiver $(\mathrm{X}-\mathrm{X})$; (b) $\mathrm{Y}$-source $\mathrm{X}$ receiver; (c) X-source, $\mathrm{Y}$-receiver; (d) $\mathrm{Y}$-source, $\mathrm{Y}$-receiver. Note the nearly constant amplitude of the XX and YY panels since we tried to remove the AVO effect from the polarization distortion. 
To demonstrate the problems with Alford rotation for offset data, I constructed a profile at a source-receiver azimuth of $30^{\circ}$ to the $\mathrm{x}$-axis. That is, the horizontal sources and receivers are all oriented in the $\mathrm{X}$ and $\mathrm{Y}$ directions but the source-receiver azimuth is at an angle of $30^{\circ}$ to the $\mathrm{x}$-direction. Note that reflected shear waves introduce energy on the cross-term components (Figure 58). The presence of any cross-term energy is commonly understood by some interpretation to be diagnostic of azimuthal anisotropy, which is not present in this purely isotropic model. This effect is solely due to the polarization distortion resulting from the horizontal source and receiver orientations not being aligned with the source-receiver azimuthal direction. To correct for this discrepancy, I correct the amplitudes for differences in SV and SH components and rotate the data recorded along the $30^{\circ}$ azimuth to the field coordinates survey geometry. Figure 58.A shows the $\mathrm{SV}$ and $\mathrm{SH}$ amplitude data after it has been rotated $30^{\circ}$ into the $\mathrm{X}-\mathrm{Y}$ field coordinates. 

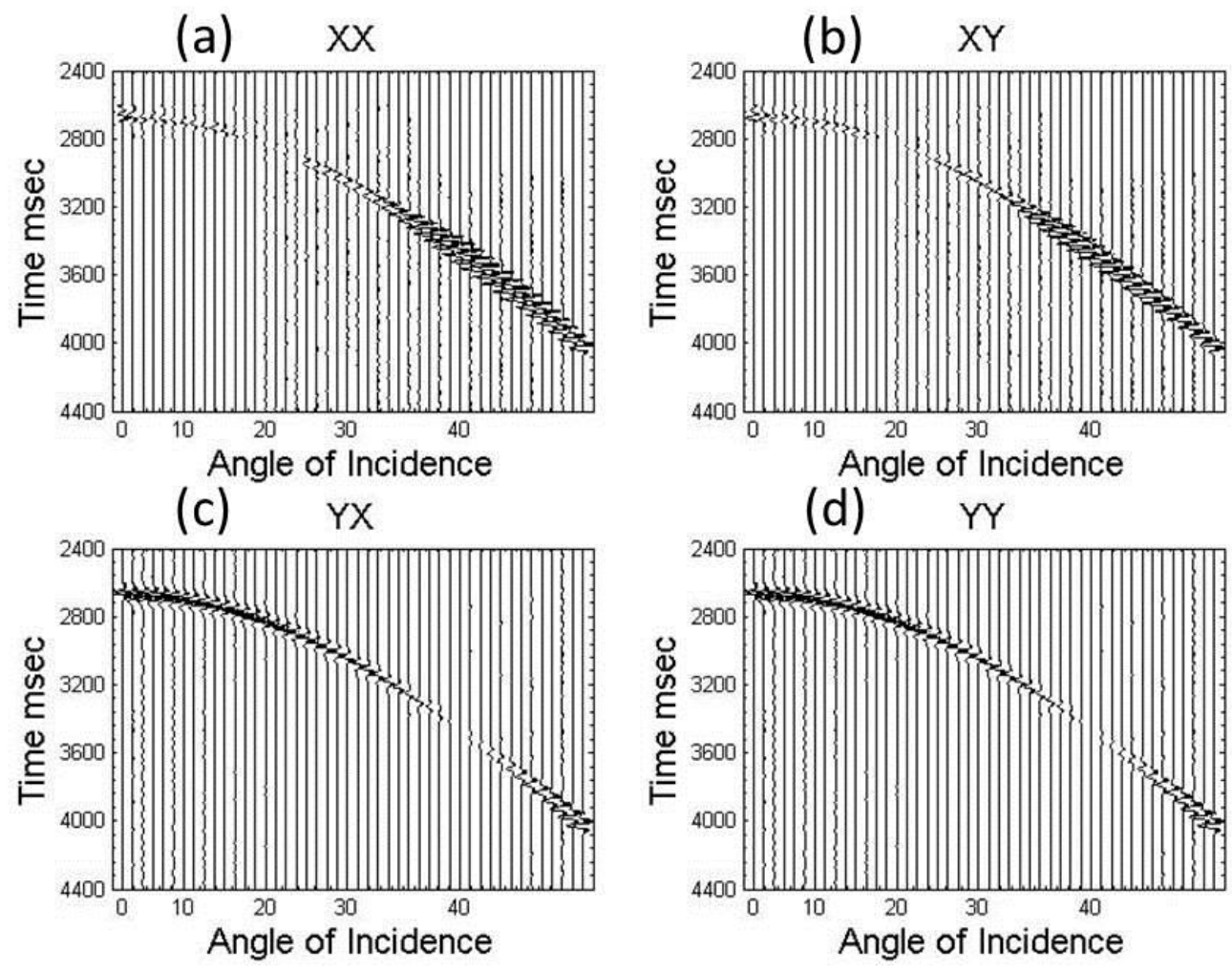

Figure58: Time-distance (offset) synthetic profile oriented at a $30^{\circ}$ to the $\mathrm{X}$ axis for a two layer isotropic model outlined in Table 1. Layer depth is at $2 \mathrm{~km}$. Horizontal axis is linear in offset, but labeled in equivalent angle of incidence. (a) data for the $\mathrm{X}$-source into the $\mathrm{X}$ receiver $(\mathrm{X}-\mathrm{X})$; (b) $\mathrm{Y}$-source $\mathrm{X}$ receiver; (c) $\mathrm{X}$ source, Y-receiver; (d) Y-source, Y-receiver. X and Y receiver source showing a source-receiver azimuth of $30^{\circ}$. Note the cross term energy in the (XY) and (YY) resulting solely from difference in SV-SV and SH-SH reflectivity and the lack of alignment between the source-receiver azimuth and the orientation of the horizontal source and receivers. 

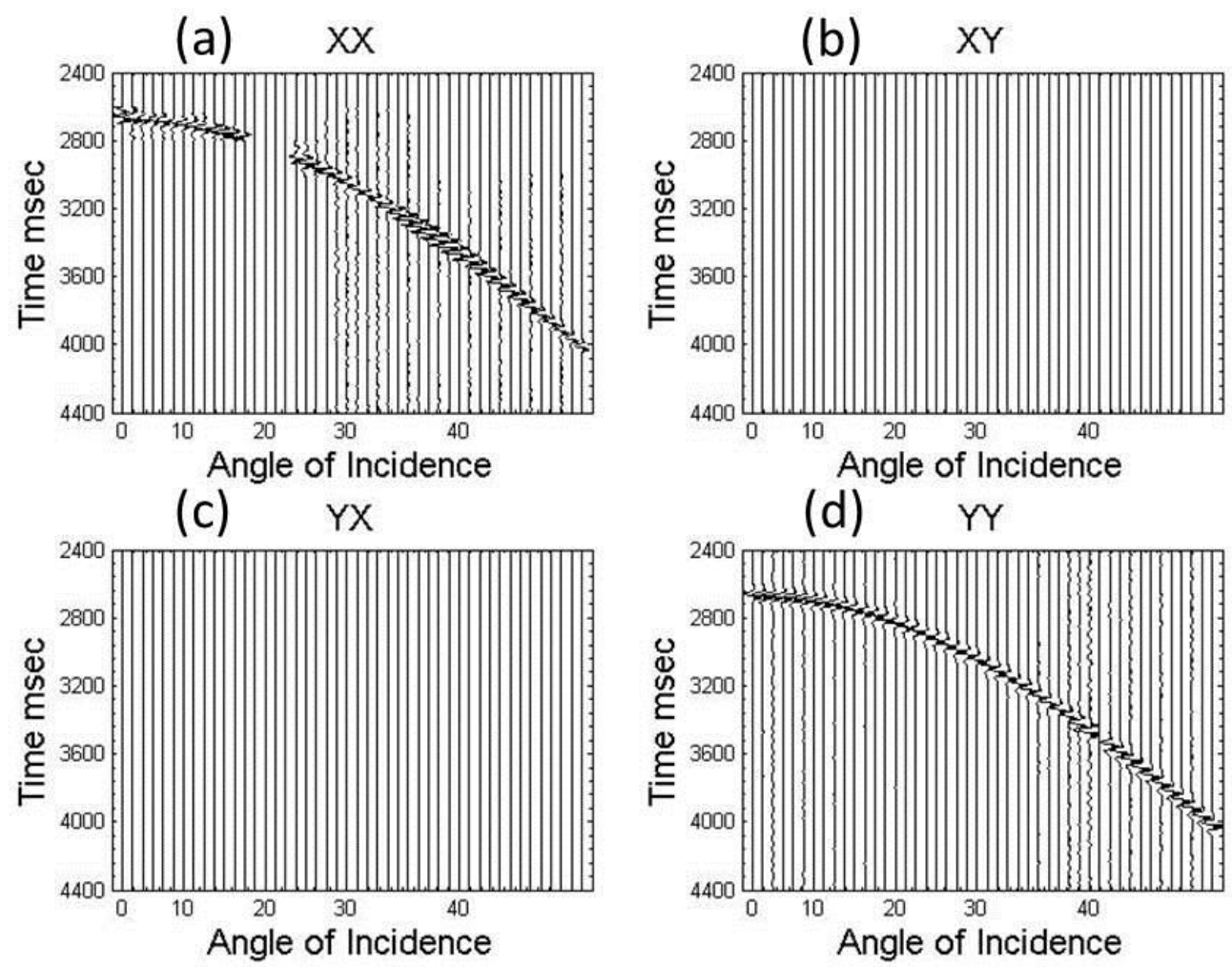

Figure58. A: Polarization correction and rotation of $30^{\circ}$ oriented profile (Figure 58) to an $\mathrm{X}$-oriented profile applied to data in Figure 58. The polarization correction removes the AVO different effect on the SV and SH. Layer depth is at $2 \mathrm{~km}$. Horizontal axis is linear in offset, but labeled in equivalent angle of incidence. (a) data for the $\mathrm{X}$-source into the $\mathrm{X}$ receiver $(\mathrm{X}-\mathrm{X})$; (b) $\mathrm{Y}$-source $\mathrm{X}$ receiver; (c) $\mathrm{X}$-source, $\mathrm{Y}$-receiver; (d) $\mathrm{Y}$-source, $\mathrm{Y}$-receiver. The result is the offset-corrected Alford rotation angle of $30^{\circ}$. Note the removal of the cross term energy in the (XY) and (YY) resulting solely from difference in $\mathrm{SV}-\mathrm{SV}$ and SH-SH reflectivity (compare to Figure 57A). There is some residual cross-term energy present, but it is so minimal that it appears that all the energy is removed. 


\section{Polarization Correction for the Anisotropic Medium}

Now we expand our discussion to the lower layer being anisotropic as seen in Chapter 2. I simulated the same direct shear-wave data in a model of a anisotropic (HTI) beneath an isotropic medium using a Fourier frequency-wave number numerical modeling procedure (Mallick and Frazer, 1987). The parameters used to create the isotropic over anisotropic model are outlined in Table 5 (similar to Table 2 with different fracture strike), including the Thomsen (1986) parameters for the lower anisotropic layer.

\begin{tabular}{|c|c|}
\hline & Model Parameters \\
\hline Layer 1 (Isotropic) & $\mathrm{V}_{\mathrm{P}}=3.0 \mathrm{~km} / \mathrm{sec}$ \\
$\mathrm{V}_{\mathrm{S}}=1.5 \mathrm{~km} / \mathrm{sec}$ \\
$\rho=2.00 \mathrm{~g} / \mathrm{cc}$ \\
\hline Layer 2 (HTI) & $\mathrm{V}_{\mathrm{P}}(0)=4.0 \mathrm{~km} / \mathrm{sec}$ \\
& $\mathrm{V}_{\mathrm{S}}(0)=2.0 \mathrm{~km} / \mathrm{sec}$ \\
$\epsilon=0.02$ \\
$\delta=0.10$ \\
$\gamma=0.05$ \\
& Fracture Strike $=\mathrm{N} 30^{\circ} \mathrm{E}$ \\
$\rho=2.2 \mathrm{~g} / \mathrm{cc}$ \\
\hline
\end{tabular}

Table 5: Properties of an anisotropic synthetic media, where $V_{P}$ is the compressional wave velocity, $V_{S}$ is the shear wave velocity. $V_{P}$ and $V_{S}$ are the vertically propagating P-wave and S-wave velocities in the HTI media, respectively. $\varepsilon$, $\delta$, and $\gamma$ are the exact Thomsen parameters of the equivalent HTI media.

Figure 59 shows synthetic data generated for this model where we see energy in the crossterms, unlike in Figures 57 and 58 for isotropic case. In Figure 59 we can see a 
range between $20-25$ degrees for the $\mathrm{X}$-source $\mathrm{X}$ receiver the zero crossing occurs at about $23^{\circ}$ and beyond $24^{\circ}$ the synthetic data shows a "splitting" into linear and non-linear waves. For the X-source Y-receiver there is not much reflection strength below $24^{\circ}$ but greater than $24^{\circ}$ the reflection strength increases. For the $\mathrm{Y}$-source $\mathrm{X}$-receiver there is low amplitude strength between $24^{\circ}$ and $42^{\circ}$ and for the $\mathrm{Y}$-source Y-receiver the synthetic data looks similar to the X-source X-receiver. The only difference between the Y-source Y-receiver and the X-source X-receiver synthetic data is the zero crossing value and the splitting nature seems to be more pronounced on the X-source X-receiver. Figure 60 shows the results of the polarization correction applied to the synthetic data as in Figure 59. The zero crossing used for the SV and SH waves were calculated used Rüger's approximations which are $23^{\circ}$ and $50^{\circ}$ respectively. The zero-crossing for the $\mathrm{SH}$ synthetic is difficult to identify on the seismic traces because of the underlying anisotropy which causes plane wave to be distorted and difficult to image.

A simple Alford rotation scan was performed at azimuth angles from $0^{\circ}-90^{\circ}$ from using the Alford's rotation and the results are best demonstrated graphically to see where the energy is reduced and where it is maximum (Figure 61). From Figure 61, with the polarization correction applied, we observed notable differences between the original acquisition coordinates and $\mathrm{SV}$ and $\mathrm{SH}$ in the rotated (to the proper orientation) coordinates. At $0^{\circ}$ azimuth, $\mathrm{S}_{\mathrm{X}}, \mathrm{R}_{\mathrm{X}}$ is $\mathrm{SV}$ and $\mathrm{S}_{\mathrm{Y}}, \mathrm{R}_{\mathrm{Y}}$ is $\mathrm{SH}$. This subset is essentially a 2$\mathrm{D}$ acquisition experiment in the X-direction. Note that at $90^{\circ}$ azimuth (2-D acquisition in the $\mathrm{Y}$-direction), but now the $\mathrm{SH}$ data appear on $\mathrm{S}_{\mathrm{X}}, \mathrm{R}_{\mathrm{X}}$ and the $\mathrm{SV}$ data appear on $\mathrm{S}_{\mathrm{Y}}$, $\mathrm{R}_{\mathrm{Y}}$.

As the source-receiver azimuth increases from zero, crossterms $S_{X}, R_{Y}$ and $S_{Y}, R_{X}$ increase in amplitude, a maximum at $\theta=20^{\circ}$ (Figure 61), and along with $S_{X}, R_{X}$ and $S_{Y}$, $\mathrm{R}_{\mathrm{Y}}$ become a mixture of $\mathrm{SH}, \mathrm{SV}$, and P-waves. After a rotation the crossterm energy 
disappeared at $\theta=30^{\circ}$ (Figure 61) because it's equal to the fracture strike of the model. Beyond $\theta=30^{\circ}$ (Figure 61) the azimuth there is again a mixing of $\mathrm{SV}, \mathrm{SH}$ and $\mathrm{P}$ waves which is used to determine azimuthal anisotropy.

The presence of reflection energy on cross terms ( $\mathrm{Y}-\mathrm{X}$ and $\mathrm{X}-\mathrm{Y})$ (Figure 59) is generally used to suggest the presence of azimuthal anisotropy. After Alford's rotation (Figure 60) we see significant reduction in this cross-term energy on $\theta=30^{\circ}$ because we are in equivalent radial-transverse coordinate. Initial polarization distortion correction is a key initial step in the analysis, processing, and interpretation of seismic data before rotation. Given those results, one would be better positioned to deal with the other components and with other complications, such as azimuthal anisotropy. 
(a) $X-X$

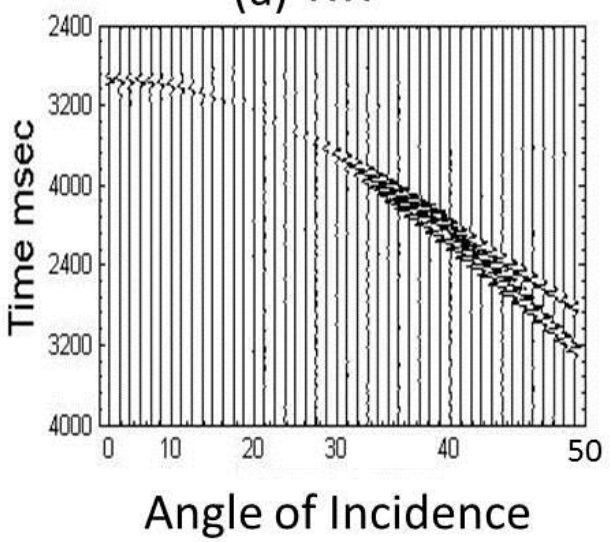

(c) $Y-X$

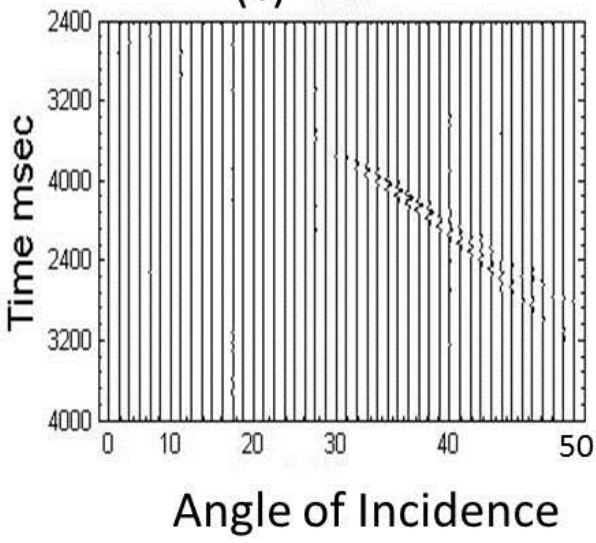

(b) $X-Y$

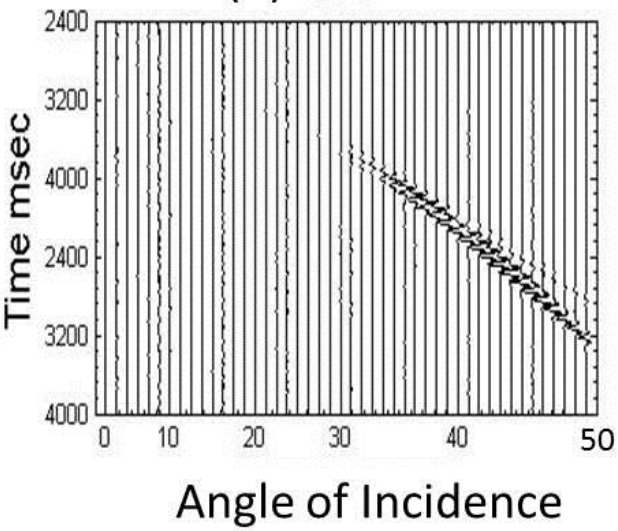

(d) $Y$ Y

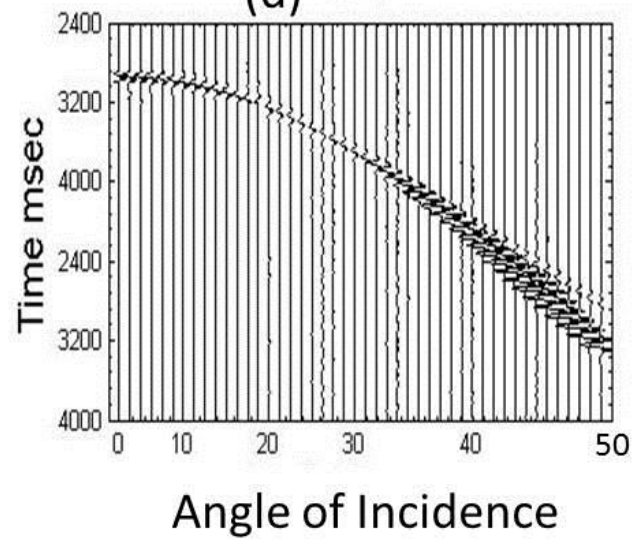

Figure 59: Synthetic data from the two layer models isotropic/ anisotropic model outlined in Table 5. The layer depth at $2 \mathrm{~km}$. Horizontal axis is linear in offset, but labeled in equivalent angle of incidence. (a) data for the $\mathrm{X}$-source into the $\mathrm{X}$ receiver (X-X); (b) Y-source X receiver; (c) X-source, Y-receiver; (d) Ysource, $\mathrm{Y}$-receiver. Note zero crossing occurs at $22^{\circ}$ for $\mathrm{X}-\mathrm{X}$ and $50^{\circ}$ for $\mathrm{Y}$ $Y$ panels with cross-term energy being present indicating anisotropy. The zero crossing on the Y-Y panel is difficult to see because of out of plane energy being present. Note the change in trace amplitude from $0^{\circ}$ to $50^{\circ}$. No polarization correction rotations have been applied. 
(a) $\mathrm{X}-\mathrm{X}$

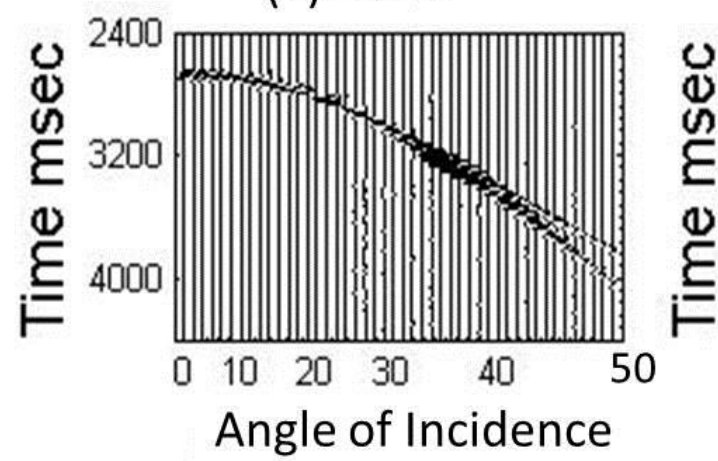

(c) $X-Y$

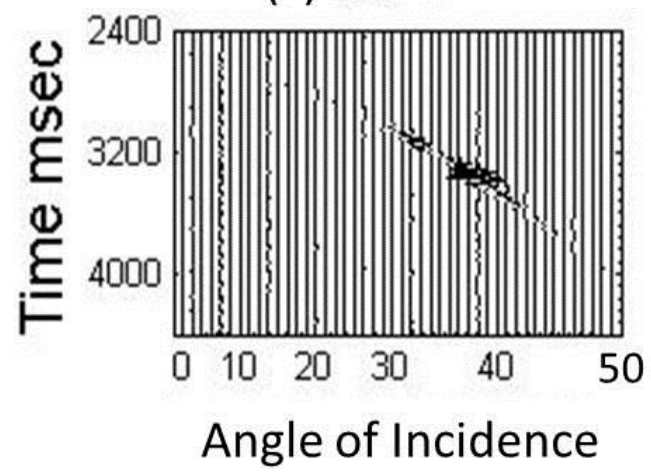

(b) $Y-X$

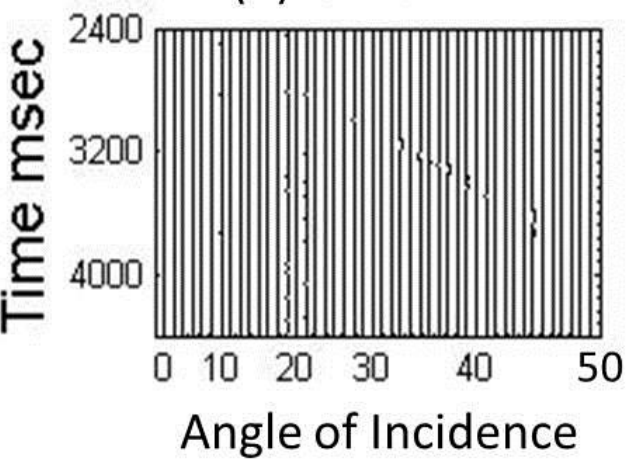

(d) $Y-Y$

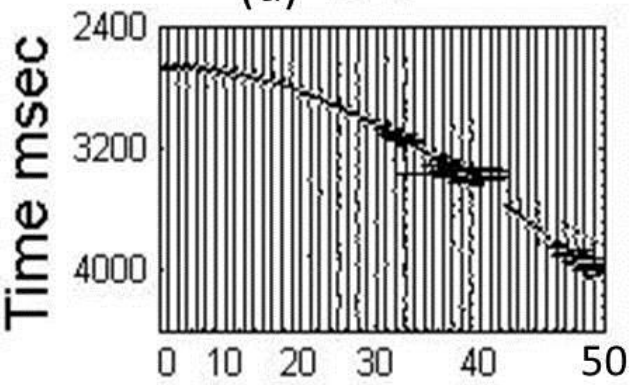

Angle of Incidence

Figure 60: Polarization correction applied to synthetic data (Figure 59) from the two layer model isotropic/ anisotropic model after polarization correction. The layer depth at $2 \mathrm{~km}$. Horizontal axis is linear in offset, but labeled in equivalent angle of incidence. (a) data for the $\mathrm{X}$-source into the $\mathrm{X}$ receiver $(\mathrm{X}-\mathrm{X})$; (b) Y-source X receiver; (c) X-source, Y-receiver; (d) Y-source, Y-receiver. Note constant amplitude of $\mathrm{X}-\mathrm{X}$ and $\mathrm{Y}-\mathrm{Y}$ panels and how the cross-term energy changes after polarization correction has been applied. Also the zero crossing $\left(50^{\circ}\right)$ on the $\mathrm{Y}-\mathrm{Y}$ synthetic seismic but other traces are also muted due to the high amplitude nature after polarization correction is applied. 

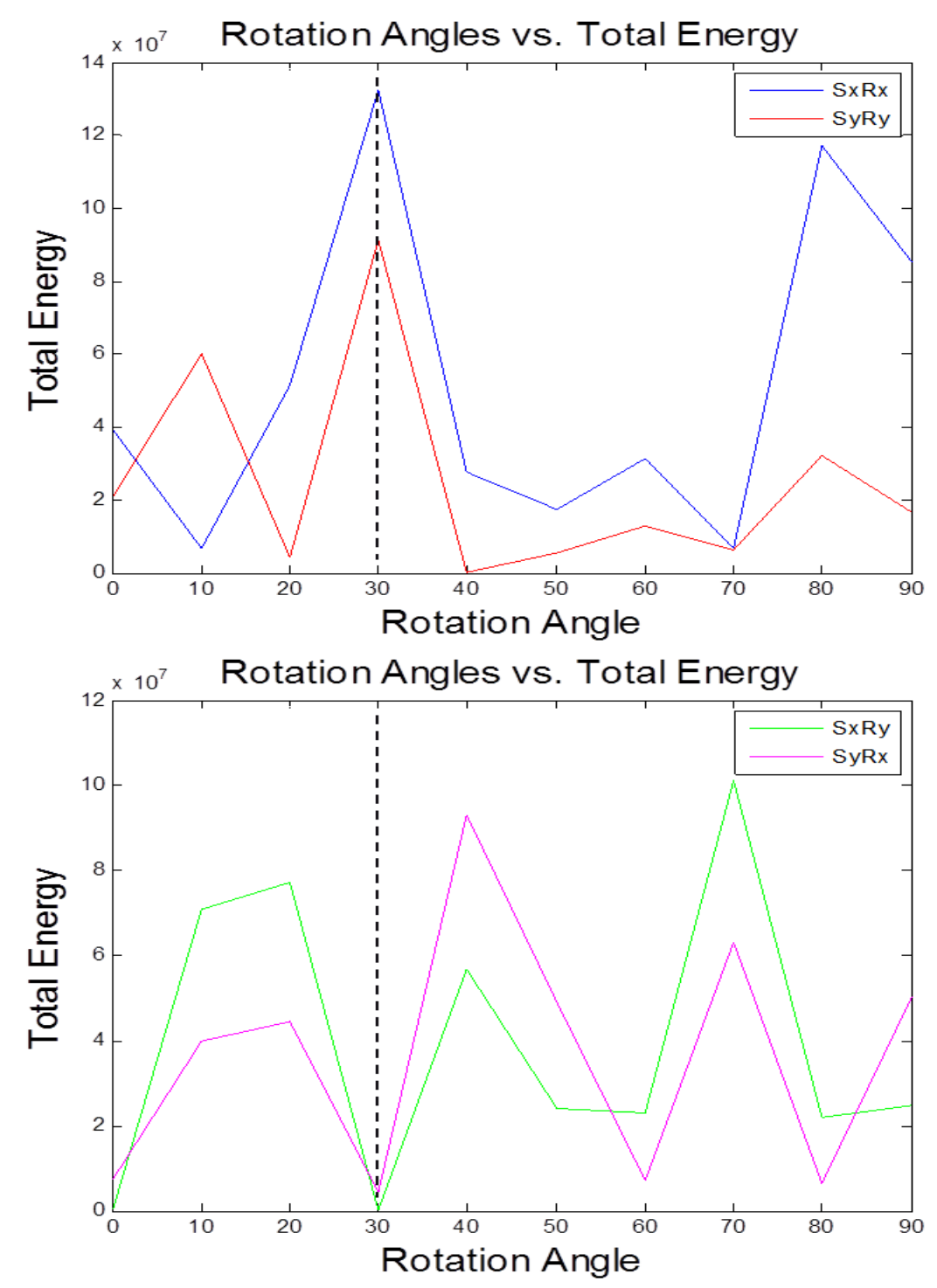

Figure 61: Diagram shows Alford rotation angle scan from $0^{\circ}$ to $90^{\circ}$ in ten degree increments to determine what angle causes the cross-term energy to disappear. The rotation angle projects these data to $\mathrm{SV}$ and $\mathrm{SH}$ coordinates consistent with the HTI geometry, and the cross-term energy is minimized. Note that we still see the presence of energy on the cross-terms because the fracture strike direction is $30^{\circ}$. The original model has a $30^{\circ}$ fracture strike direction and the plot indicates that at $30^{\circ}$ the cross-term energy is zero and the $\mathrm{X}-\mathrm{X}$ and $\mathrm{Y}-\mathrm{Y}$ receiver pairs are maximum. As noted all other rotation angles the cross-term energy is not zero meaning it's not at the correct orientation. The data is collected over a two layer models isotropic/ anisotropic model outlined in Table 5 after polarization correction. The layer depth at $2 \mathrm{~km}$. 


\section{Alford Rotation Applied to BlackBear field Oklahoma}

The polarization correction analysis was applied to the shear wave seismic data in the Black Bear survey. A single time horizon was interpreted shallow in the section. As discussed in Chapter 3, we know that the shallow section of the Black Bear survey does demonstrate significant azimuthal anisotropy. Willis et al. (1986) identified 14 survey sites that showed azimuthal anisotropy at 12 of the locations, no observed azimuthal anisotropy at only one of them and non-usable data at the other. From this small (14) sample, we can suggest that azimuthal anisotropy occurs at in at least $85 \%$ of the locations that are surveyed. Thus, the existence of azimuthal anisotropy at the location of the Black Bear survey is not a surprise.

The four horizontal component direct shear wave data were acquired with arrays of two orthogonal horizontal shear-wave sources and two orthogonal horizontal receivers. Since we do not know the orientation of the HTI anisotropy, we need to perform a rotational analysis to transform the data to the natural polarization directions. We do have

some regional knowledge of the fracture parameters from a 9C VSP and Dipole log survey conducted in a nearby Carter county, about 25 miles east of the survey area. As discussed earlier, the Brady-1 well (Figure 37) shows that the Oklahoma has a wide range of vertical fracture strikes, both locally and in depth. Thus, I focus on the interpreted horizon in the shallow section. This survey is in a structurally and tectonically complex area, so some care must be applied in extrapolating these parameters even 25 miles away from the Brady well location.

After interpretation of the shallow seismic time horizon discussed above, we apply the polarization distortion correction to the seismic data set in order to appropriately apply the modified Alford's rotation for non-zero offset data. Ideally, this 
correction overcomes the zero-offset limits of Alford Rotation and yields a valid Alfordlike rotation for non-normal angles of incidence.

The four component Alford-like rotations of horizontal components were used to estimate the optimum rotation angle and infer the fracture orientations. To estimate the angle, we assume that the optimum rotation angle is the angle that concentrates the most energy to the principal diagonal components (X-X and $\mathrm{Y}-\mathrm{Y})$. Applying the rotation algorithm using the estimated angle ideally aligns the shear wave traces from their input orientations into the natural polarization direction of the data (Shuck et al. 1996).

The source and receiver locations, shown in Figure 48 (Chapter 3), describe the single source location for the pre-stack data (shows the geometry Figure 62) with receivers yielding the traces displayed in increasing source-receiver offset. Note that there is a mixing of the $\mathrm{P}, \mathrm{SV}$ and $\mathrm{SH}$ waves in all four source-receiver $(\mathrm{X}-\mathrm{X}, \mathrm{Y}-\mathrm{X}, \mathrm{X}-\mathrm{Y}$, Y-Y) combinations.

The SV and SH amplitude distortion correction is then applied to the original data before applying the modified Alford's rotation. Figure 63 shows the corrected pre-stack data from this source location, with receiver traces ordered in increasing source-receiver offset. Note the change in apparent reflection coherency of each of the pre-stack gathers and the apparent improvement in data quality for the $\mathrm{X}-\mathrm{X}$ and $\mathrm{Y}-\mathrm{Y}$ of the orientations. The Y-X and X-Y cross-term energy does appear to be less coherent at the shallow reflector level.

After correction to radial-transverse coordinates, the reflections around the interpreted reflecting horizon $(1.1 \mathrm{sec})$ should be maximized in $\mathrm{S}_{\mathrm{X}}, \mathrm{R}_{\mathrm{X}}$ and $\mathrm{S}_{\mathrm{Y}}$, and $\mathrm{R}_{\mathrm{Y}}$ components but minimize on $\mathrm{S}_{\mathrm{X}}, \mathrm{R}_{\mathrm{Y}}$ and $\mathrm{S}_{\mathrm{Y}}, \mathrm{R}_{\mathrm{X}}$ components. Shallow shear-wave reflections are apparent in the $\mathrm{SH}\left(\mathrm{S}_{\mathrm{Y}} \mathrm{R}_{\mathrm{Y}}\right)$ data. Reflection events are different and more difficult to identify in $\mathrm{SV}\left(\mathrm{S}_{\mathrm{X}} \mathrm{R}_{\mathrm{X}}\right)$. These shallow reflections appear only over a limited 
offset range in SV (a narrow-offset corridor just outside the strong near-offset ground roll). The first arrival appears to be muted. Also SV-SV has a zero crossing so no data are observed at mid-range offsets, limiting its usefulness for analysis.

Figure 64 shows application of the proposed Alford rotation to $10^{\circ}$. I also display the $30^{\circ}$ azimuth rotation (Figure 65 ) and $70^{\circ}$ azimuth rotation (Figure 66). As discussed above, HTI azimuthal anisotropy is the shallow section may be expected, as evidence by observations at the Texaco Brady 1 borehole.

To better apply the Alford rotation analysis scan for amplitude (energy) levels at a variety of rotation angles. Energy at the $1100 \mathrm{msec}$ reflection zone is highlighted in yellow on the seismic sections. The scan of rotations were applied from $0^{\circ}-90^{\circ}$ in $10^{\circ}$ increments. The results of this rotation angle scan are summarized in Figure 67. With the polarization correction applied, we observe notable differences in energy between the original acquisition coordinates and $\mathrm{X}-\mathrm{X}$ and $\mathrm{Y}-\mathrm{Y}$ components in the rotated (to the proper orientation) coordinates. At $0^{\circ}$ azimuth relative to field coordinates, $\mathrm{S}_{\mathrm{X}}, \mathrm{R}_{\mathrm{X}}$ is $\mathrm{SV}$ and $\mathrm{S}_{\mathrm{Y}}, \mathrm{R}_{\mathrm{Y}}$ is $\mathrm{SH}$. This subset is essentially equivalent to a 2-D acquisition experiment in the X-direction.

As the rotation angle increases from zero, $\mathrm{S}_{\mathrm{X}}, \mathrm{R}_{\mathrm{Y}}$ and $\mathrm{S}_{\mathrm{Y}}, \mathrm{R}_{\mathrm{X}}$ increase in amplitude, to a maximum at $\theta=30^{\circ}$ (Figure 67). This is assumed to be the natural fracture orientation. Recall that preliminary examination of the observed reflection polarizations in Chapter 3 suggested a natural polarization direction of about $45^{\circ}$. After this rotation analysis, the crossterm energy at $\theta=30^{\circ}$ (Figure 67) is minimal and the diagonal terms are at a local maximum, suggesting that the "fracture strike" is $30^{\circ}$. Beyond $\theta=30^{\circ}$ (Figure 67) the azimuth there is again a mixing of energy from all modes. The Black Bear data suggest high levels of anisotropy in the entire shallow section, consistent with 
observations at the Brady-1 well as well as the Willis et. al (1986). Polarization distortion correction helps for the correct application of the offset correcting. 

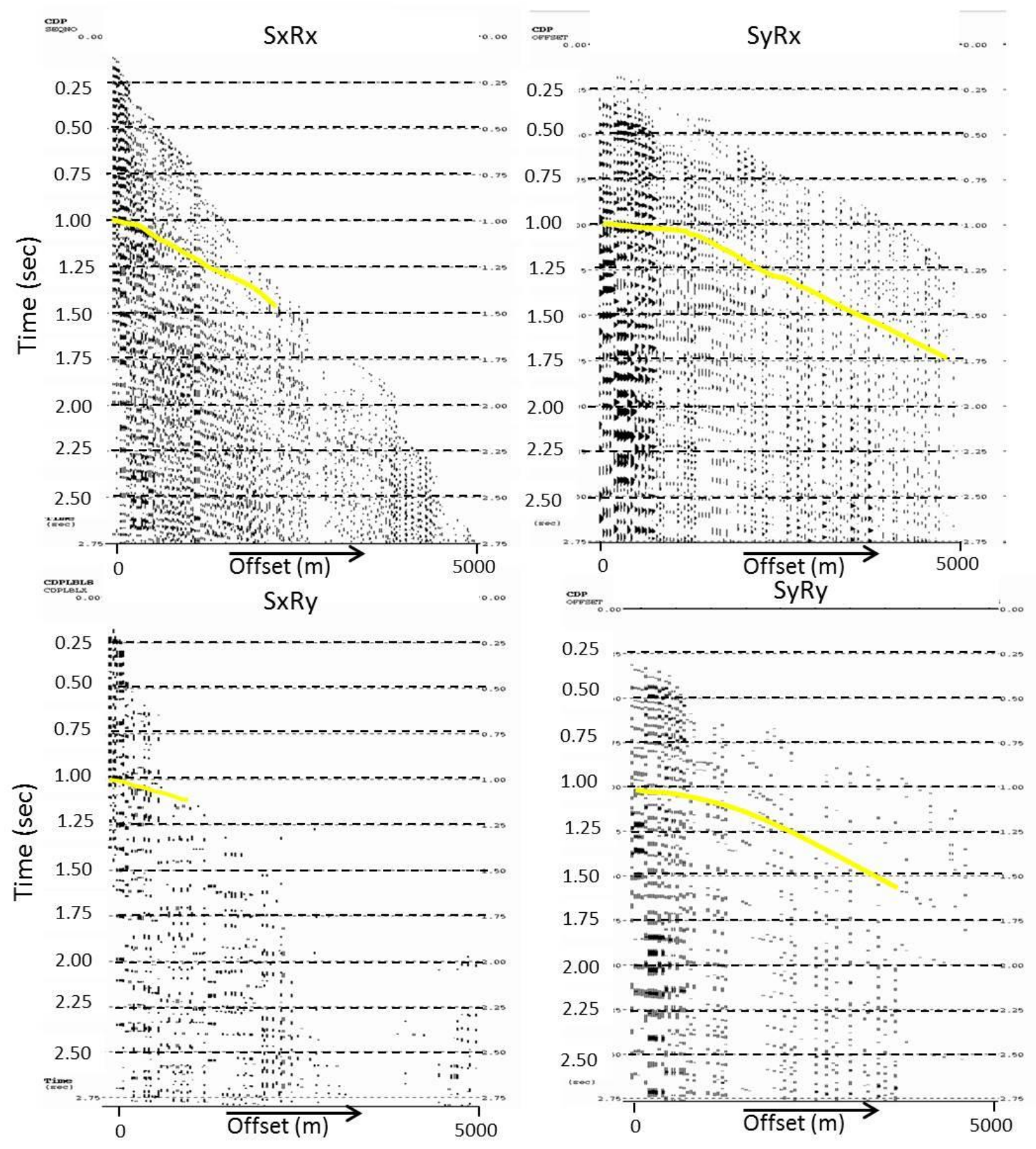

Figure 62: Original field data showing $\mathrm{X}$ and $\mathrm{Y}$ source $-\mathrm{X}$ and $\mathrm{Y}$ receiver and zone of interpreted horizon (highlighted in yellow) is at $1100 \mathrm{msec}$. Note the noisy nature of the field data before the polarization correction and Alford rotations have been applied. 

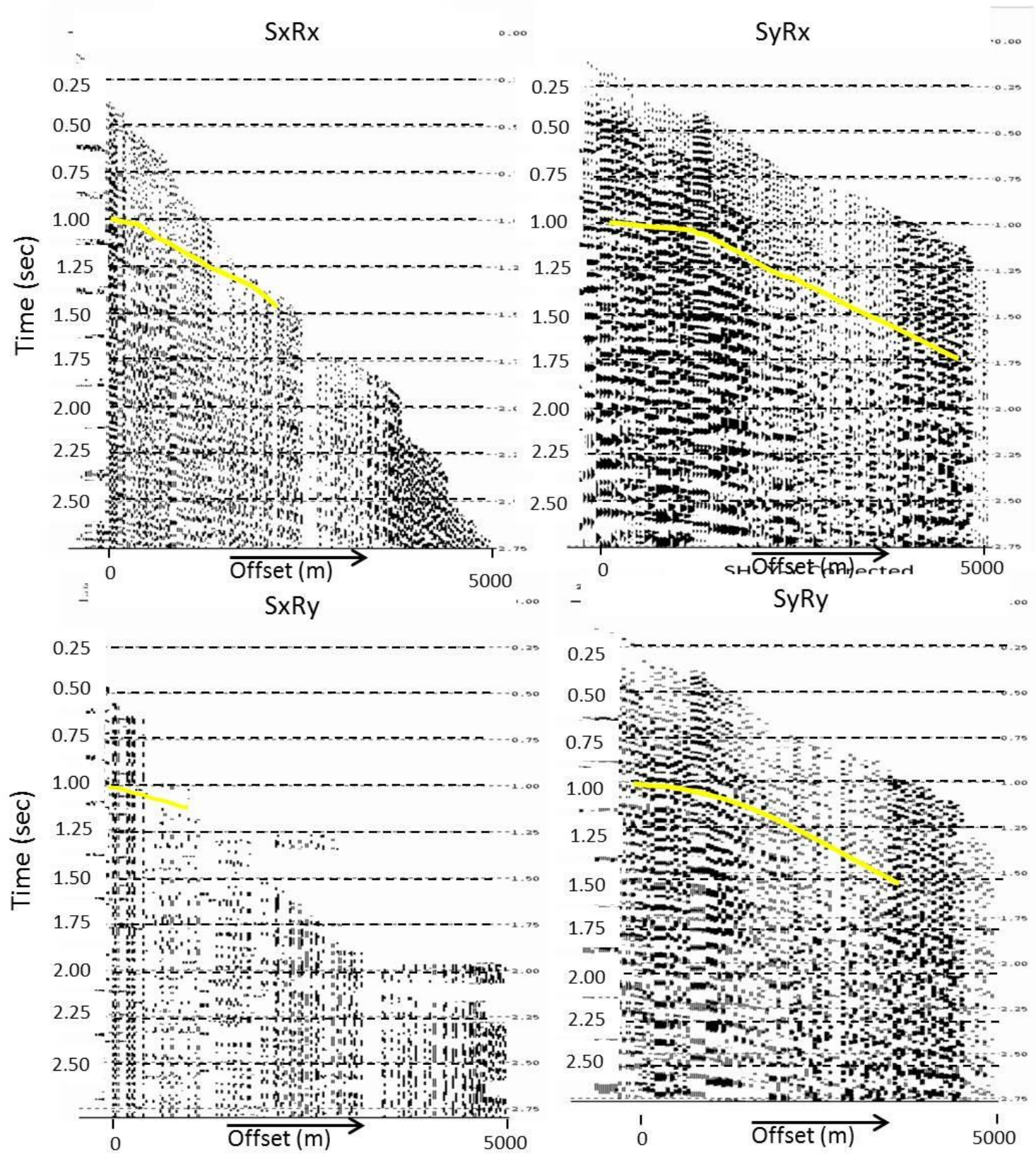

Figure 63: Polarization correction applied to the original field data showing $X$ and $Y$ source $-\mathrm{X}$ and $\mathrm{Y}$ receiver and zone of interpreted horizon is at $1100 \mathrm{msec}$. Note the major difference in the continuity of the beds and the amplitudes, between the SxRx, SyRy and the SxRy and SyRx data. No NMO correction applied. 


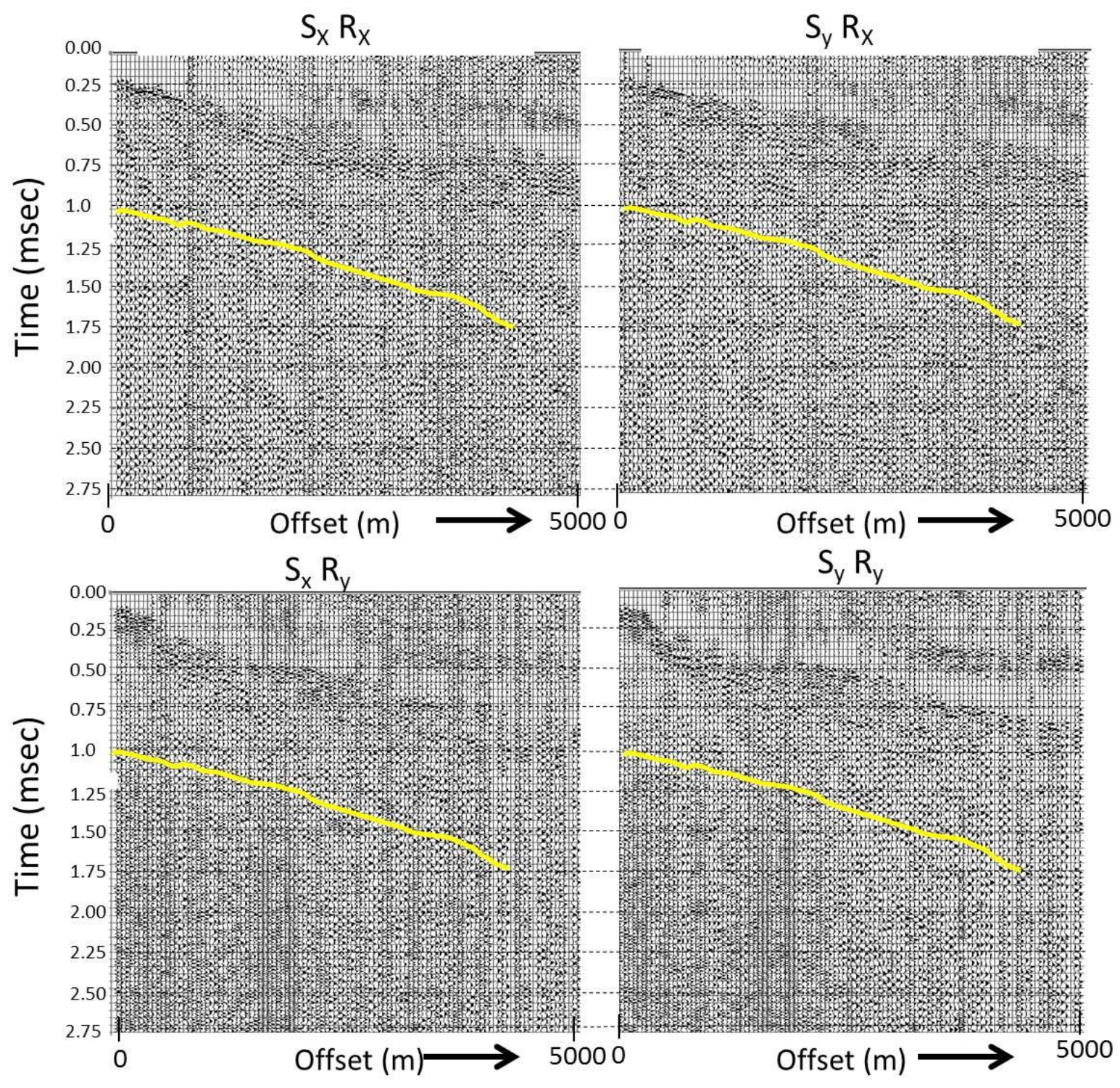

Figure 64: Polarization correction is applied to the seismic panels (Figure 63) and an Alford rotation azimuth scan of $10^{\circ}$. Note there is no major difference in bed continuity and amplitudes. The data is very noisy partly due to the high levels of azimuthal anisotropy that dominates the reflected $\mathrm{S}$ waves. 


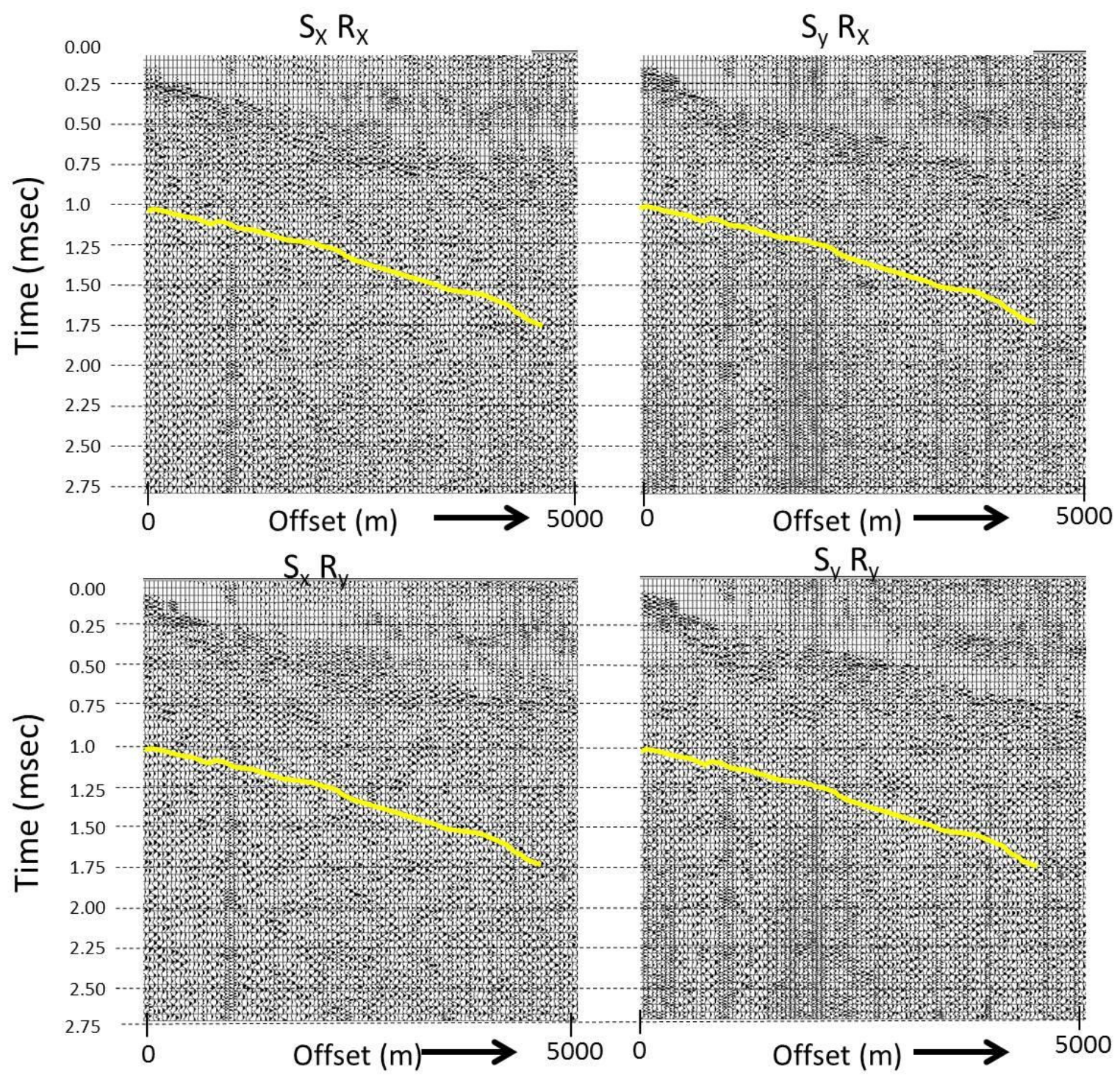

Figure 65: Polarization correction is applied to the seismic panels and an Alford rotation azimuth scan of $30^{\circ}$. Note there is no difference in the continuity of the beds and the amplitudes. The data is very noisy due to the high levels of azimuthal anisotropy that dominates the reflected $\mathrm{S}$ waves. All the azimuth scans look very noisy due to the high degree of anisotropy in the shallow section. 


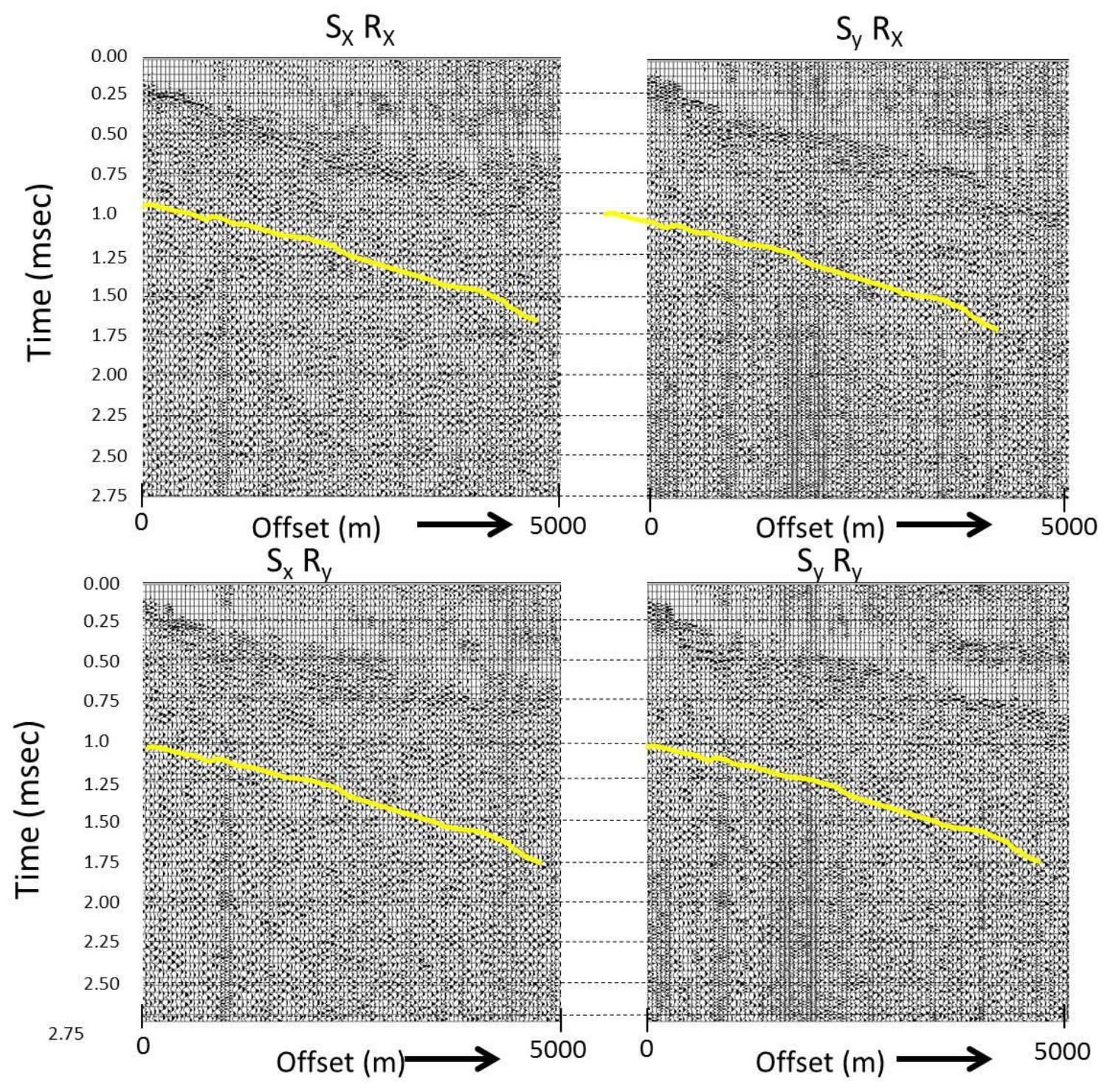

Figure 66: Polarization correction is applied to the seismic panels and an Alford rotation azimuth scan of $70^{\circ}$. Note the major difference in the continuity of the beds and the amplitudes. The data is very noisy due to the high levels of azimuthal anisotropy that dominates the reflected $\mathrm{S}$ waves. 

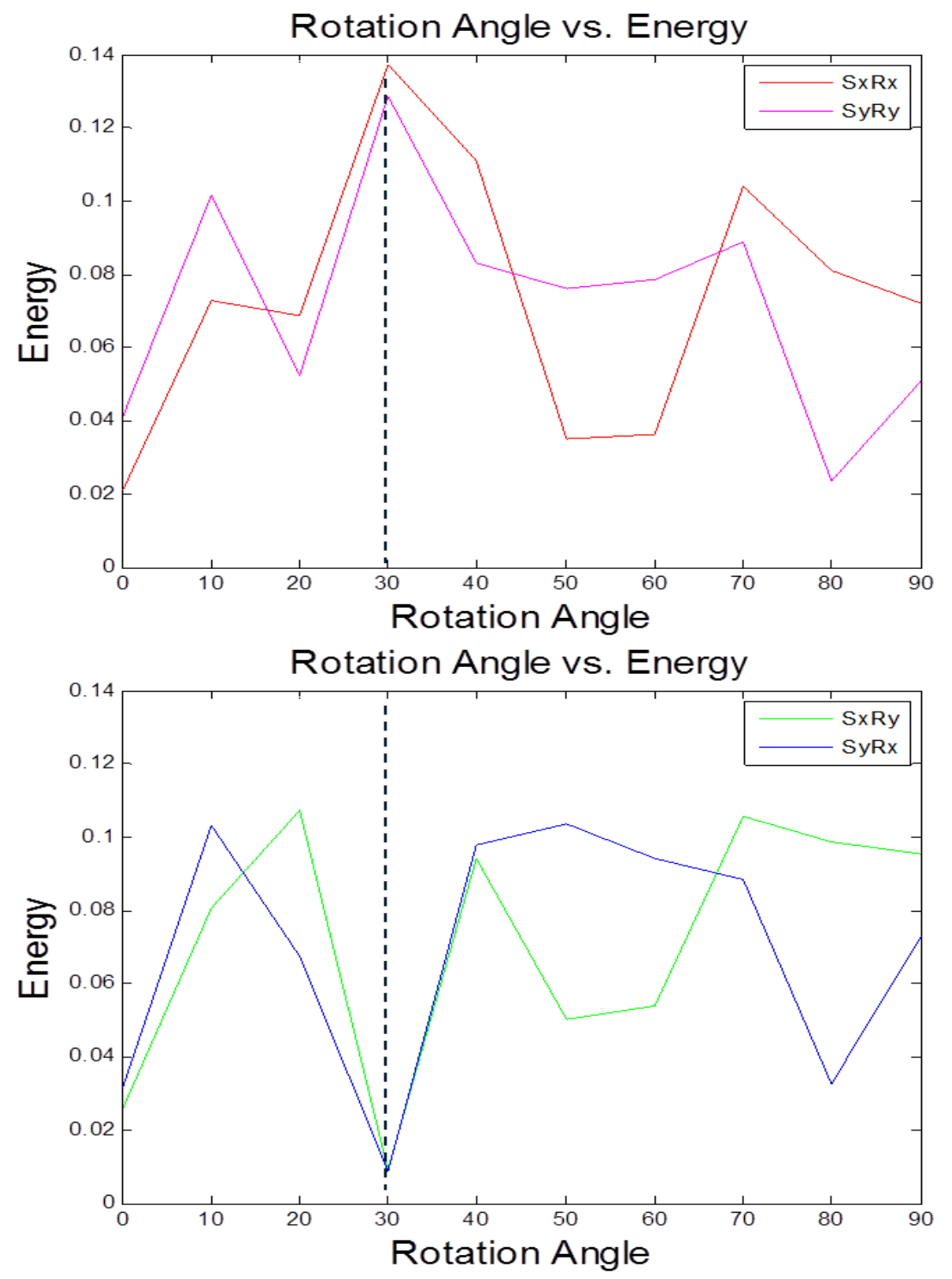

Figure 67: Scan of field data with X (east) and Y (north) sources with X (east) and Y (north) receivers, in the original field coordinates after applying the offset corrected Alford's rotation and polarization correction to all the data. Rotations have been applied using $\theta$ in ten degree increments. Correction is based on reflection geometry to the $1100 \mathrm{msec}$ reflector. There is energy on the cross-terms which is indicative of anisotropy. The values of gamma are so large that they do affect the reflection process. Cross-term energy is minimum at $30^{\circ}$ and $\mathrm{X}-\mathrm{X}$ and $\mathrm{Y}-\mathrm{Y}$ receiver pairs are maximum, which is the fracture strike at this depth. Note all other rotation angles the cross-term energy is present indicate anisotropy. 


\section{CHAPTER 5: DISCUSSION AND CONCLUSION}

My research resulted in a proposed that extends the potential application of Alford's (1987) rotation analysis beyond the current limitation of using only normal incidence reflections to include a wide range of incidence angles and source-receiver offsets. This result, along with details of its evolution and the tests of its validity,

provides a successful resolution of the originally proposed hypothesis 'to develop a complete method of polarization distortion analysis of synthetic S-wave data to replicate information available in $9 \mathrm{C}-3 \mathrm{D}$ data'.

In my research, I examined how the reflection process alters the polarization of a direct shear waves recorded at the surface, even in purely isotropic media. It was determined that this polarization distortion effect is due solely to the orientation of horizontal source and horizontal receiver elements not being aligned with the sourcereceiver azimuthal directions. The difference in the individual SV-SV and SH-SH reflection coefficients at non-zero incidence angles determines where and to what degree the polarization is altered because of the misalignment of instrument orientations and locations within a survey design. This misalignment is a feature of most 3D seismic data land acquisition designs currently employed in hydrocarbon exploration.

A correction which normalizes the differences in the SV-SV and SH-SH reflectivity with offset, however, is required. In order to remove this misalignment and distortion, I explored a simplification of the SV and SH reflectivity responses, along with a possible correction proposed by Lyons (2006). We now have a viable correction to apply to account for the survey geometry effect on shear wave polarization (prior to polarization analysis for fracture characterization).

Anisotropy presents a particular challenge and opportunity in understanding the ever expanding shale gas plays. On land seismic data, some have approached the existing limitations by restricting the data to very small offsets. Others have rotated horizontal 
components into S1 and S2 directions extracted from nearby VSP studies. Still others have worked entirely in SV-SV and SH-SH components.

Azimuthal anisotropy effects can be recognized and analyzed by monitoring the reflection signal (in radial transverse coordinates) from the top and the base of the fractured reservoir interval as a function of offset and azimuth. There are many important issues to be addressed in the effective utilization of 9-C-3-D data set, such as the Sycamore field Oklahoma and in particular multicomponent seismic data in general. At this point, removing polarization distortion from the reflection data is important in extracting fracture characterization hence rotation of the field coordinate data is essential in understanding azimuth variation for polarization distortion.

Hence focusing on the evolution of seismic technologies, including those operationally limited to onshore environments. We endeavor to determine necessary processing adaptations steps needed to derive maximum benefit. In particular, the application of techniques developed for land S-wave seismic from controlled polarization 9C data. This is crucial for addressing fracture analysis and reservoir properties from seismic anisotropy observations. Consequently, a method must be developed that utilizes a 3D 3C surface seismic data set to arrive at concurring seismic attributes and interpretations that may be extracted from the S-wave data.

\section{RECOMMENDATIONS}

Further work for expanding my results is to allow for variations in anisotropy with depth. This will require a method to include layer stripping rotational analysis, probably utilizing a matrix rotation algorithm designed specifically for multi-azimuthal 3D S-wave data. Previous published work has been tested on a simulated anisotropic data and applied to on industry-acquired data sets. There are multiple methods of layerstripping analysis were developed. In investigating polarization distortion analysis of both synthetic and pure S-wave data. They have all be limited to the normal incidence 
assumption of the conventional Alford rotation. They need to be re-examined utilizing the results of the research that will allow all of the available data to be used. numeric models. 


\section{Glossary}

3C data: Seismic reflection data acquired with a conventional seismic source, three component geophone receiver.

4C data (Marine): Seismic reflection data acquired offshore using an airgun source, three component geophone and one hydrophone receiver.

4C rotation: A four component rotation algorithm utilizing the four horizontal components (source $\mathrm{x}$ and $\mathrm{y}$ and receiver $\mathrm{x}$ and $\mathrm{y}$ ) to arrive at the principal axes of an anisotropic medium.

9C data: Seismic reflection data acquired on land using three components of source (controlled polarization) and three-component geophone receiver.

S-wave: A reflected wave generated by a controlled polarization source, such as multiaxis vibroseis.

Polarization: Direction of particle motion to the direction of wave propagation 


\section{References}

Aki, K. and P. G. Richards, 2002, Quantitative seismology, 2nd ed.: University Science Books

Alford, R. M., 1986, Shear data in the presence of azimuthal anisotropy: Dilley, Texas: 56th Ann. Internat. Mtg., Soc. Expl. Geophys., Expanded Abstracts, 476-479.

Amsden, T.W., 1979, Welling Formation, new name for Uper Ordovician unit in easten Oklahoma (formerly called "Fernvale"): American Association of Petroleum Geologist Bulletin, v. 63, p. 1135-1138.

Amaral, H.L., 2001, Shear-wave Azimuthal AVO Analysis, Vacuum Field, New Mexico, p. 41.

Amsden, T.W., 1980, Hunton Group (Late Ordovician, Silurian and Early Devonian), in the Arkoma Basin Oklahoma: Oklahoma Geological Survey Bulletin 129, 136 p.

Amsden, T.W., 1989, Depositional and post-depositional history of middle Paleozoic (Late Ordovician through Early Devonian) strata in ancestral Anadarko Basin, in Johnson, K. S., ed., Anadarko Basin Symposium, 1988: Oklahoma Geological Survey Circular 90, p. 143-146

Amsden, T.W., and Sweet, W. E., 1983 Upper Bromide Formation and Viola Group (Middle and Upper Ordovician) in eastern Oklahoma: Oklahoma Geological Survey Bulletin 132, 76 p.

Baars, D.L., Maples, C. G., Ritter, S.M., and Ross, G. A., 1991, Redefinition of the Pennsylvanian Virgilian Series in Kansas (abs.): Geological Society of America Abstracts with Program, 1990 Annual Meeting, p. 844.

Becker, F.D., Perelberg, I.A., Seismic Detection of Subsurface Fractures 
Beckham, W.E., 1996, Seismic anisotropy and natural fractures from VSP and borehole sonic tools - a field study: Geophysics, 61, 456-466.

Booth, D. C. and S. Crampin (1985). "Shear-wave polarizations on a curved wavefront at an isotropic free surface." Geophysical Journal of the Royal Astronomical Society 83(1): 31-45.

Bowman, J. R., and M. A. Ando, 1987, Shear-wave splitting in the upper mantle wedge above the Tonga subduction zone: Geophys. J.R. Astron. Soc., 88, 25-41.

Carpenter, B. N., and Evans, M. C., 1991, Comparisons of the Arbuckle Group at Wilburton field, Latimer County, with N.E. Alden field, Caddo County, Oklahoma, in Johnson, K.S., ed., Arbuckle Group core workshop and field trip: Oklahoma Geological Survey Special Publication SP 91-3, p. 111-132

Cipriani, Dan, 1963, General geological sections of Oklahoma and northern Arkansas: Oklahoma City Geological Society

Crampin, S., 1983a, Effects of anisotropy on reflectivity: 53rd Ann. Internat. Mtg, Session:RW2.8, Soc. of Expl. Geophys. — 1983b, Shear wave polarizations: A plea for three-component recording: 53rd Ann. Internat. Mtg, Session:S12.7, Soc. of Expl. Geophys.

Crampin, S., 1985, Evidence for aligned cracks in the earth's crust: First Break 03, no. 3. $12-15$

Crampin, S., 1986, Shear Waves Revealed: Extensive Dilatancy Anisotropy Confirmed

Davis, H. G., and Northcutt, R. A., 1991, Anadarko Basin, in Gluskoter, H. J., Rice, D. D., and Taylor, R. B., eds., Economic geology, U.S.: Geological Society of America, The Geology of North Amerca, v. P-2, p. 325-338.

Gaiser, J. E., 1997, 3-D Converted shear wave rotation with layer stripping: United States Patent 5,610,875 
Gaiser, J. E., 1999, Applications for vector coordinate systems of converted waves obtained by multicomponent 3-D data: 31st Annual Offshore Technology Conference, OTC10985.

Gumble, J. E., 2006, Complete Anisotropic analysis of three component seismic data related to the marine environment and comparison to nine component land seismic data, Ph.D. Dissertation, University of Texas at Austin.

Haas, E.A., 1981, Structural analysis of a portion of the Reagan fault zone, Murray County, Oklahoma: Shale Shaker, v. 21, p. 93-105.

Ham, W.E., and J.L. Wilson, 1967, Paleozoic epeirogeny and orogeny in the central United States: Am. Jour. Sci., v. 265, p. 352-407.

Harding, T.P., Gregory, R.F. and Stephens, L.H., 1983. Convergent Wrench Fault and positive flower structure

Ardmore Basin, Oklahoma. In: A.W. Bally (Editor), Seismic Expression of Structural Styles. Am. Assoc. Pet. Geol., Studies in Geology Series, No. 15, Vol. 3, pp. 4.213.

Hester, T. C., Schomoker, J, W., and Sahl, H. L., 1990, Log-derived regional source-rock characteristics of Woodford Shale, Anadarko Basin, Oklahoma: U.S.: Geological Survey Bulletin 1866-D, 38 p.

Hills, J. M., and Kotlowski, F.E., coordinates, 1983, Southwest and southwest MidContinent region (SSMC), in Lindberg, F.A., ed., Correlation of stratigraphic units in North America (COSUNA): American Association of Petroleum geologist.

Hoffman, P., K.C. A. Burke, and J. E Dewey, 1974, Aulacogens and their genetic relation to geosynclines with a Proterozoic example from Great Slave Lake, Canada in R.H. Dott, Jr., and R.H. Shaver, eds., SEPM Spec. Pub. 19, p. 38-55.

Hoenig, D. and D. Moebius (1991). "Direct visualization of monolayers at the air-water interface by Brewster angle microscopy." The Journal of Physical Chemistry 95(12): 4590-4592. 
Johnson, H. D., VSP Detection of Fracture-Induced Velocity Anisotropy

Johnson, K. S., 1988, general geologic framework of the field-trip area, in Johnson K. S., ed., Shelf-to-basin geology and resources of Pennsylavanian strata in the Arkoma Basin and frontal Ouchita Mountains of Oklahoma: Oklahoma geological Survey Guidebook 25, p. 1-5.

Kleehammer, R.S., 1991, Conodont biostratigraphy of later Mississippian shale sequences, south-central Oklahoma: unpublished M.S. thesis, University of Oklahoma, Norman, $135 \mathrm{p}$.

Knode, T. L., Reaser, D. F., and Schieber, Juergen, 1993, Geological aspects of the Devonian Misener/Sylamore Sandstone in the United States (abs.): American Association of Petrolum Geologist Bulletin, v. 77, p. 139.

Krohn, C. E., 1988, Computer modeling of P-SV waves: 58th Ann. Internat. Mtg, Session: S13.5, Soc. of Expl. Geophys.

Lambert, M. W., in press, Revised Upper Devonian and Lower Mississippian nomenclature in Kansas, in Baars, D. L., ed., Changes in stratigraphic nomenclature in Kansas: Kansas Geological Survey Bulletin 230.

Lynn, H. B., Bates, C. R., Simon, K. M., and van Dok, R., 1995, The effects of azimuthal anisotropy in P-wave 3-D seismic: $65^{\text {th }}$ Ann. Internat. Mtg., Soc. Expl. Geophys., Expanded Abstracts, $293-296$.

Lynn, H. B., Thomsen, A. L., 1986, Reflection Shear-Wave Data along the Principal Axes of Azimuthal Anisotropy

Lyons, E. S., 2006, Polarization rotation upon reflection of direct shear waves in purely isotropic media, MS Thesis, University of Texas at Austin.

Mallick, S. and L. N. Frazer, 1987, Practical aspects of reflectivity modeling, Geophysics, 52, 1355-1364. 
Mankin, C.J., coordinator, 1986, Texas-Oklahoma tectonic region (TOT), in Lindberg, F. A., ed., Correlation of stratigraphic units in North America (COSUNA): American Association of Petroleum Geologist.

Martin, M.A., and T.L. Davis, 1987, Shear-wave birefringence: A new tool for evaluating fractured reservoirs: The Leading Edge, Society of Exploration Geophysicists, October, 1987, p. 22-28.

Mavko, Gary; Mukerji, Tapan; Dvorkin, Jack (2003), The Rock Physics Handbook, Cambridge University Press

Musgrave, M.J.P., 1970, Crystal acoustics: Holden Day.

Ohanian, V., and Beckham, W. E., 1992, Depth-variant fracture orientation from multicomponent VSP data: 62nd Internat. Mtg., Soc. Explor. Geophys., Expanded Abstracts, 351-54.

Pilant, W. L., 1979. Elastic Waves in the Earth, Elsevier, Amsterdam.

Peron, J., 1990, Estimation of fracture direction from zero-offset VSP by two- and fourcomponent rotation: 60th Internat. Mtg., Soc. Expl. Geophys., Expanded Abstracts, 1, 2-3.

Rai, C, S., Hanson, E. K., 1986, Shear-Wave Birefringence: A Laboratory Study

Rüger, A., 1997a, P-wave reflection coefficients for transversely isotropic model with vertical and horizontal axis of symmetry: Geophysics, 62, 713-722.

Rüger, A., 2001, Analytic insight into shear-wave AVO for fractured reservoirs: Advances in anisotropy, International Workshop on Seismic Anisotropy (7IWSA). 
Rueger, A., 2002, Reflection coefficients and azimuthal AVO analysis in Anisotropic media: SEG.

Schoenberg, M., and F. Muir, 1989, A calculus for finely layered anisotropic media: Geophysics, 54 , no.5, 581-589.

Shearer, P. M., 1999, Introduction to seismology: Cambridge University Press

Sheriff, R. E. and L. P. Geldart, 1995, Exploration seismology, 2nd ed.: Cambridge University Press.

Shuck, E. L., T. L. Davis, and R. D. Benson, 1996, Multicomponent 3-D characterization of a coalbed methane reservoir: Geophysics, 61, 315-330.

Shuey, R. T., 1985, A simplification of the Zoeppritz-equations: Geophysics, 50, 609614. Errata in GEO-50-9-1522.

Simmons, J., and M. Backus, 2001, Shear waves from 3-D-9-C seismic reflection data: Have we been looking for signal in all the wrong places? The Leading Edge, 20, 604-612.

Simmons, J., 2004, Multicomponent Prestack Modeling in Isotropic/Anisotropic Media: Annual Meeting, CSEG, S083.

Spratt, R. S., N. R. Goins, and T. J. Fitch, 1993, Pseudo-shear - The analysis of AvO, in Backus, M. M., ed., Offset-dependent reflectivity - theory and practice of AVO analysis, 37-56. Soc. of Expl. Geophys.

Suneson, H.N., 1996, The Geology of the Ardmore Basin in the Lake Murray State Park Area Oklahoma: Oklahoma Geological Survey

Tanner, J.H., III, 1967, Wrench fault movements along Washita Valley fault, Arbuckle Mountain area, Oklahoma: AAPG Bull., v. 51, p. 126-141. 
Tatham, R. H., M. D. Matthews, K. K. Sekharan, C. J. Wade, and L. M. Liro, 1992, A physical model study of shear-wave splitting and fracture intensity: Geophysics, $57,647-652$.

Tatham, R. H. and M. D. McCormack, 1991, Multicomponent Seismology in Petroleum Exploration: SEG, Edited by E. B. Nietzel and D. F. Winterstein.

Taylor, D. B. \& Crampin, S., 1978. Seismic surface waves in anisotropic media: propagation in a homogeneous piezoelectric halfspace, Proc. R. Soc. A, 364, 161179.

Thomsen, L., 1986, Reflection Seismology in Azimuthally Anisotropic Media

Thomsen, L., 1986, Weak elastic anisotropy: Geophysics, V.51, No 10, p. 1954-1966.

Thomsen, L., 1988, Reflection seismology over azimuthally anisotropic media. Geophysics, 53, 304-313.

Thomsen, L., 2001, Vector recomposition of seismic 3-D converted-wave data: United States Patent 6,292,754 B1

Tsvankin, I., 2001, Characterization of dipping fractures in a transversely isotropic background. Geophysical Prospecting, 2004, 52, 1-10.

Thomsen, L., 2002, Understanding seismic anisotropy in exploration and exploitation, SEG

Wielchowsky, C.C., and O.E. Gilbert, Jr., 1982, Style, timing, and mechanism of basement-involved versus detached Paleozoic deformation, southeastern Oklahoma and northern Texas (abs.): Geol. Soc. America, Abs. with Prog., v. 14, n. 3, p. 140.

Wilcox, R.E., T.P. Harding, and D.R. Seely, 1973, Basic wrench tectonics: AAPG Bull., v. 57, p. $74-96$. 
Willis, H.A., Rethford, G.L., Bielanski, E., 1986, Azimuthal Anisotropy: Occurrence and Effect on Shear-Wave Data Quality

Wilson, J. L., Fritz, R. D., and Medlock, P. L., 1991, The Arbuckle Group- relationships of core and outcrop analyses to cyclic stratigraphy and correlations, in Johnson, K. S., ed., Arbuckle Group core workshop and field trip: Oklahoma Geological Survey Special Publication SP 91-3, p. 133-143.

Winterstein, D. F., and Meadows, M. A., 1991a, Changes in shear wave polarization with depth in Cymric and Railroad Gap oil fields: Geophysics, 56, 1349-1364. 1991b, Shear-wave polarization and subsurface stress directions at Lost Hills Field: Geophysics, 56, 1331-1348.

Yilmaz, O., 2001, Seismic Data Analysis: Soc. of Expl. Geophys., 02 edition. 